Supporting Information for:

\title{
Examining the Generality of Metal-Ligand Cooperativity Across a Series of First-Row Transition Metals: Capture, Bond Activation, and Stabilization
}

John J. Kiernicki, ${ }^{a}$ Matthias Zeller, ${ }^{b}$ and Nathaniel K. Szymczak ${ }^{a *}$

\begin{abstract}
${ }^{a}$ Department of Chemistry, University of Michigan, Ann Arbor, Michigan 48109, United States
${ }^{b}$ H.C. Brown Laboratory, Department of Chemistry, Purdue University, West Lafayette, Indiana 47907, United States

*To whom correspondence should be addressed: nszym@umich.edu
\end{abstract}


General Considerations .....

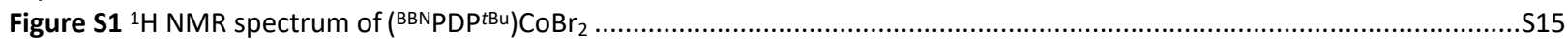

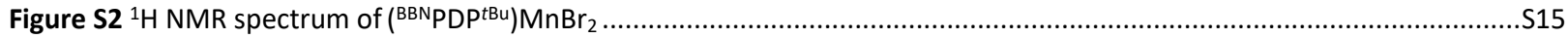

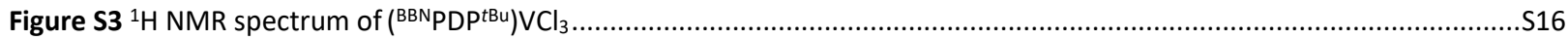

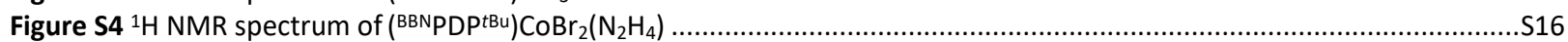

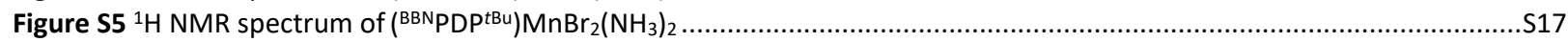

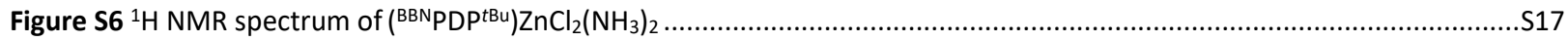

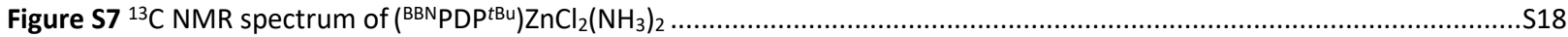

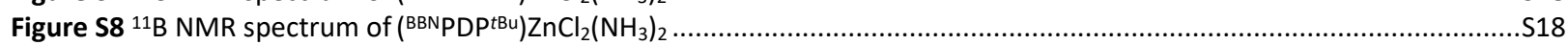

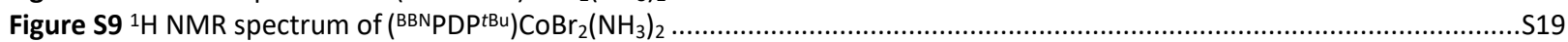

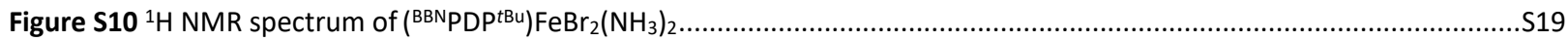

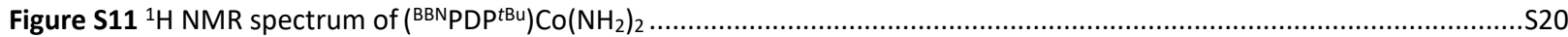

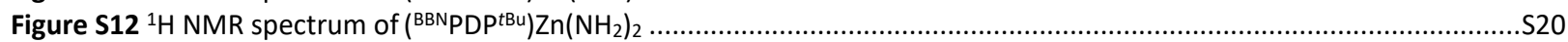

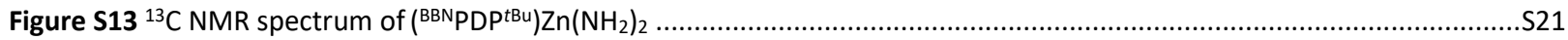

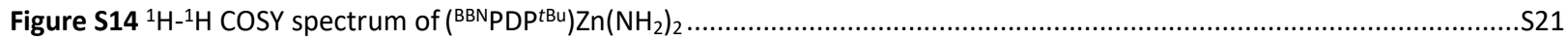

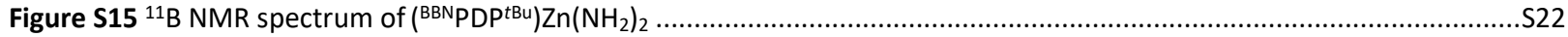

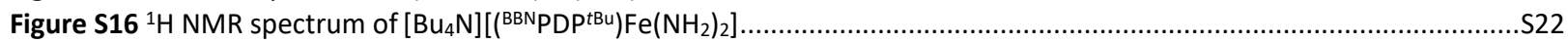

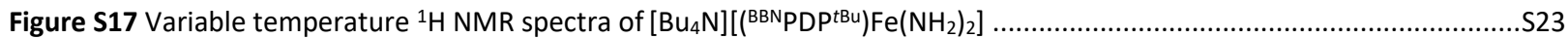

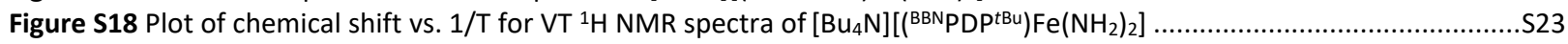

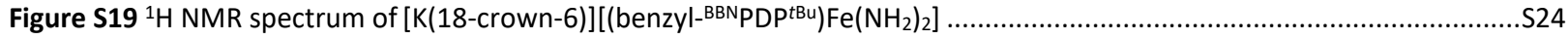

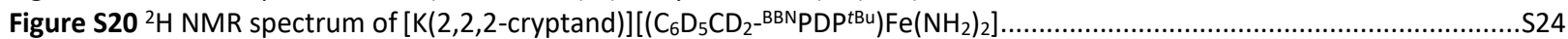

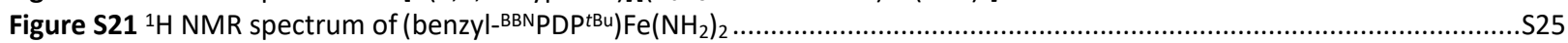

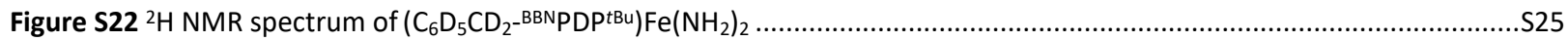

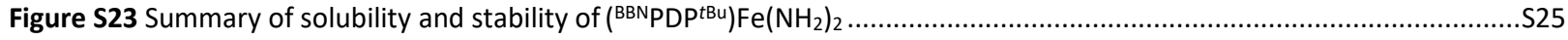

Figure S24 ${ }^{1} \mathrm{H}$ NMR spectra (THF) of oxidation of (BBNPDPtBu) Fe $\left(\mathrm{NH}_{2}\right)_{2}$ with $\mathrm{PhICl}_{2}$ and authentic (BBNPDPtBu$) \mathrm{FeCl}_{2}\left(\mathrm{NH}_{3}\right)_{2} \ldots \ldots \ldots . . . \mathrm{S} 26$

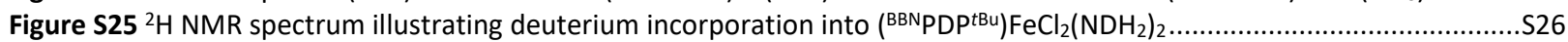

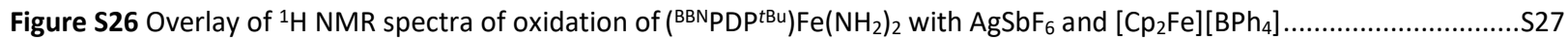

Figure S27 ${ }^{1} \mathrm{H}$ NMR spectra $\left(\mathrm{C}_{6} \mathrm{H}_{4} \mathrm{Cl}_{2}\right)$ of oxidation of (BBNPDPtBu) Fe $\left(\mathrm{NH}_{2}\right)_{2}$ with $\mathrm{Ag}^{+}$and authentic ( $\left.{ }^{\mathrm{BBNPDP}} \mathrm{Bu}^{\mathrm{Bu}}\right) \mathrm{FeCl}_{2}\left(\mathrm{NH}_{3}\right)_{2} \ldots \ldots . . . \mathrm{S} 27$

Figure S28 ${ }^{1} \mathrm{H}$ NMR spectra $\left(\mathrm{CH}_{2} \mathrm{Cl}_{2}\right)$ of oxidation of ( $\left.{ }^{\mathrm{BBN} P D P t B u}\right) \mathrm{Fe}\left(\mathrm{NH}_{2}\right)_{2}$ with $\mathrm{Ag}^{+}$and authentic ( $\left.{ }^{\mathrm{BBNPDPP}}{ }^{\mathrm{BBu}}\right) \mathrm{FeCl}_{2}\left(\mathrm{NH}_{3}\right)_{2} \ldots \ldots \ldots . . . \mathrm{S} 28$

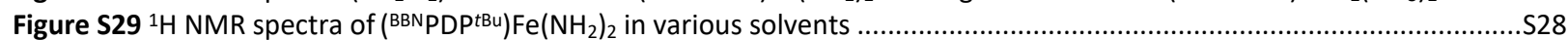

Figure $\mathrm{S}^{3}{ }^{1} \mathrm{H}$ NMR spectra of $\left({ }^{\mathrm{BBN} P D P t B u}\right) \mathrm{Fe}\left(\mathrm{NH}_{2}\right)_{2}$ in DMF when treated with excess tetrabutylammonium bromide.............S29

Figure S31 ${ }^{1} \mathrm{H}$ NMR spectra of ( $\left.{ }^{B B N P D P t B u}\right) \mathrm{FeBr}_{2}$, ( $\left.{ }^{\mathrm{BBN} P D P}{ }^{\mathrm{Bu} u}\right) \mathrm{FeBr}_{2}\left(\mathrm{NH}_{3}\right)_{2}$, and $\left({ }^{\mathrm{BBN} P D P}{ }^{\mathrm{Bu}}\right) \mathrm{FeBr}_{2}\left(\mathrm{~N}_{2} \mathrm{H}_{4}\right)$ in DMF (decomposition). S29

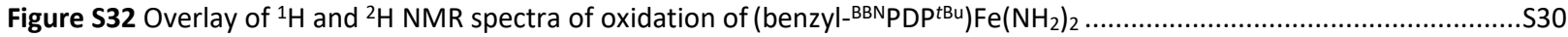

Figure S33 Overlay of ${ }^{1} \mathrm{H}$ NMR spectra of oxidation of (benzyl-BBNPDPtBu)Fe $\left(\mathrm{NH}_{2}\right)_{2} \mathrm{~W} /$ and $\mathrm{w} / \mathrm{o}$ dihydroanthracene..................S31

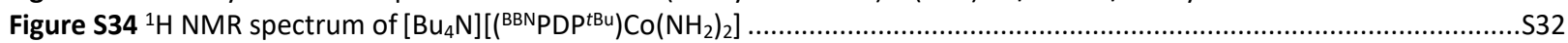

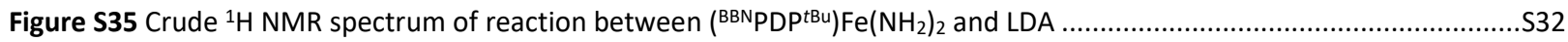

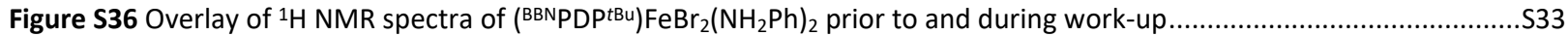

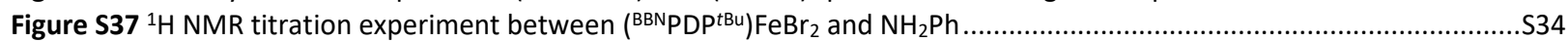

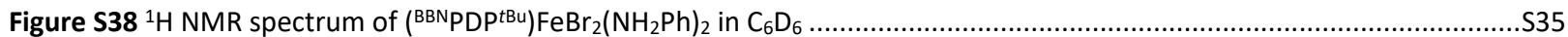

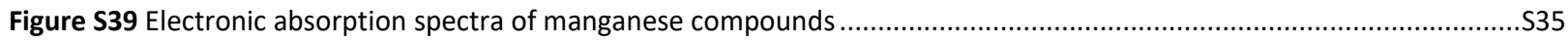

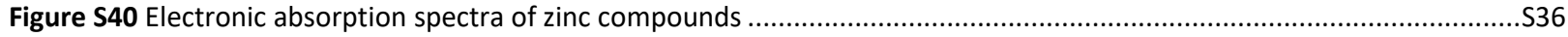

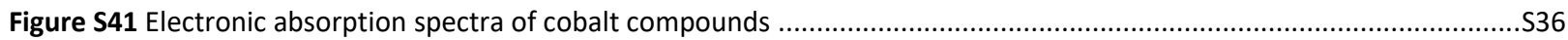

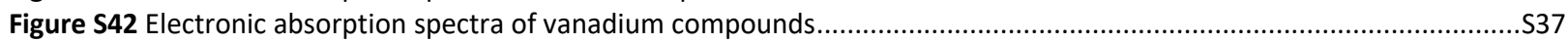

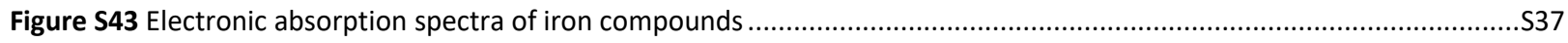

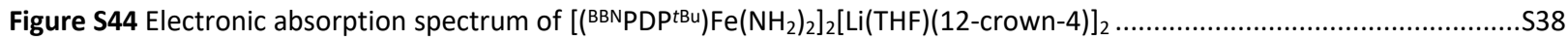

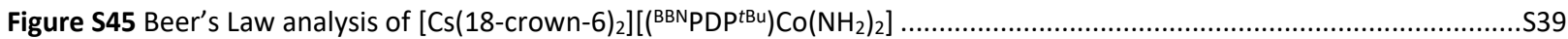

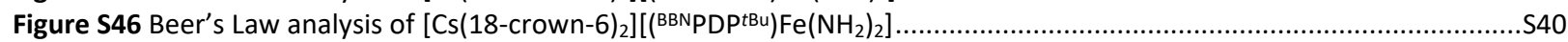

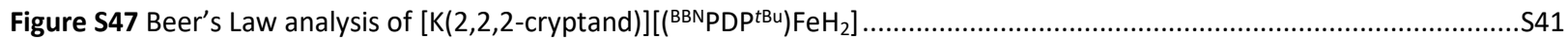

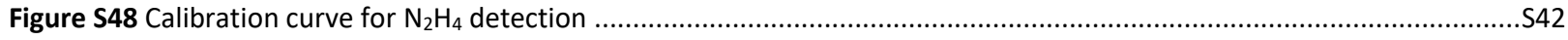

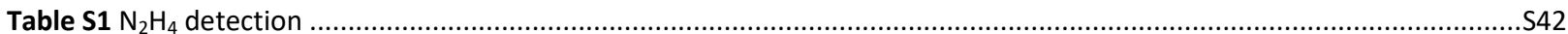

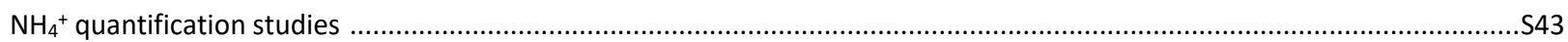

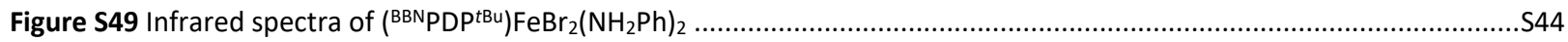

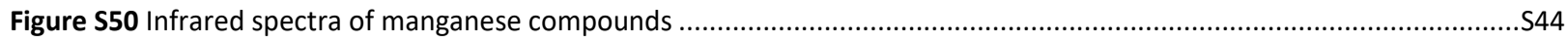

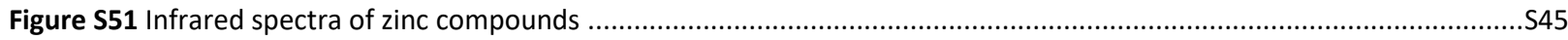

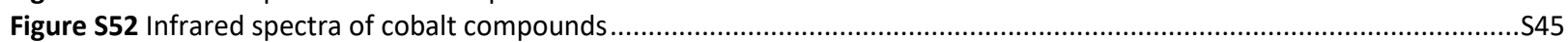

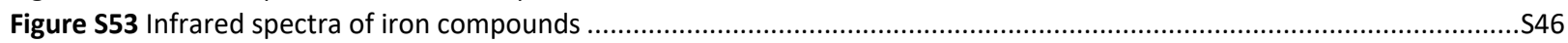

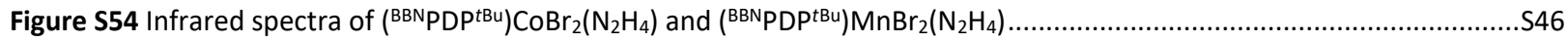




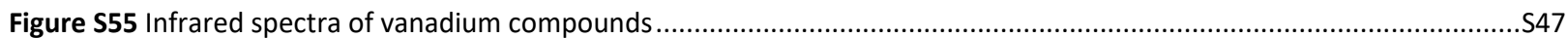

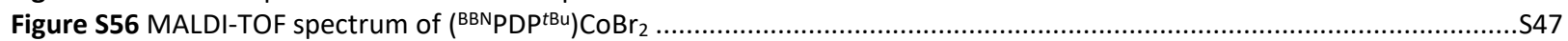

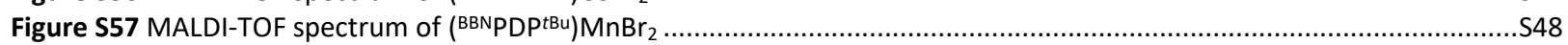

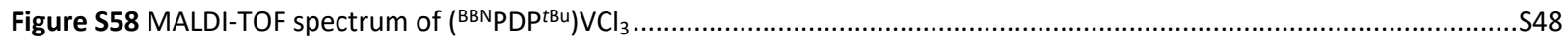

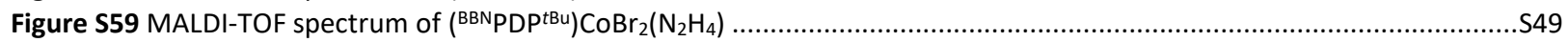

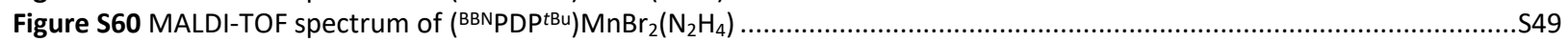

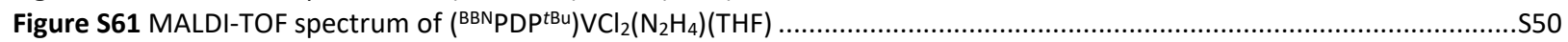

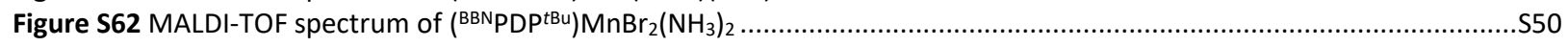

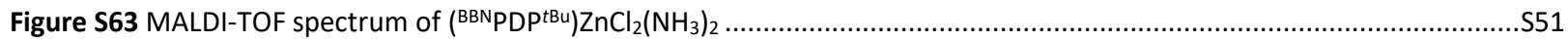

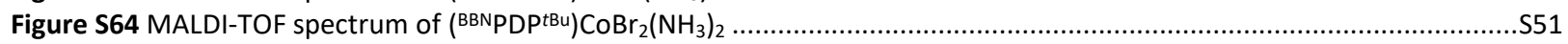

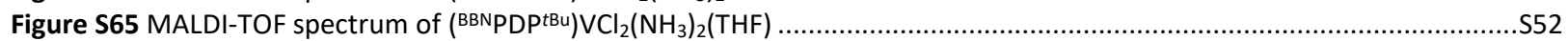

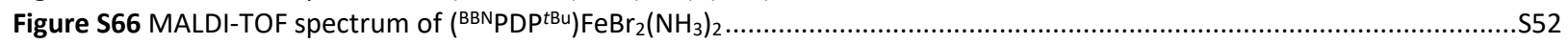

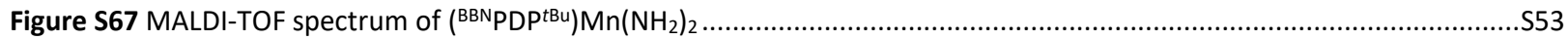

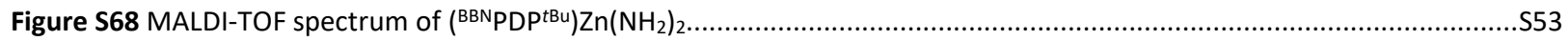

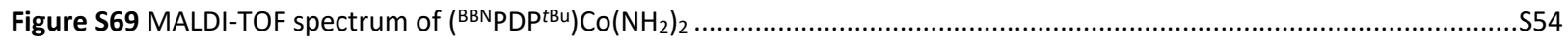

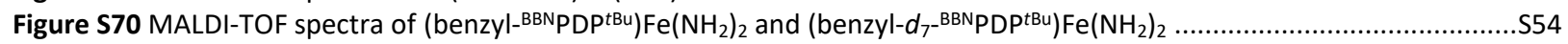

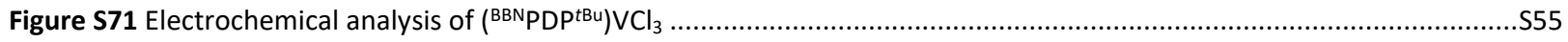

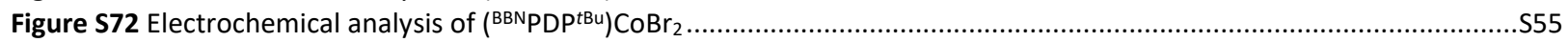

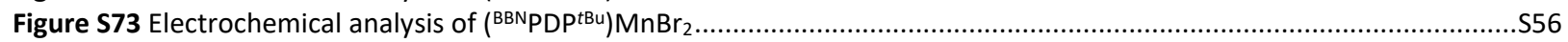

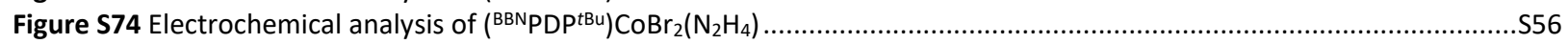

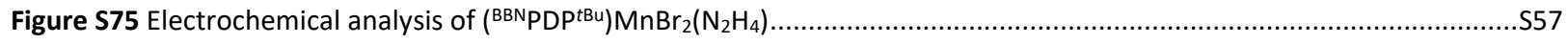

Figure S76 Electrochemical analysis of ( $\left.{ }^{B B N P D P}{ }^{\mathrm{BBu}}\right) \mathrm{Fe}\left(\mathrm{NH}_{2}\right)_{2}$ in DMF w/ and w/o dihydroanthracene......................................557

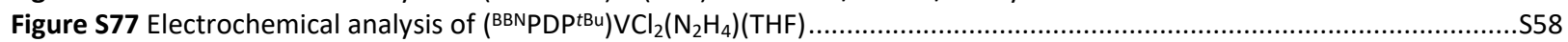

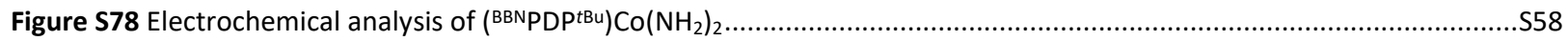

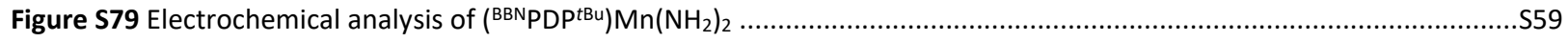

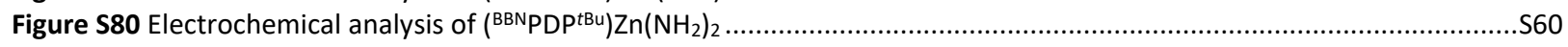

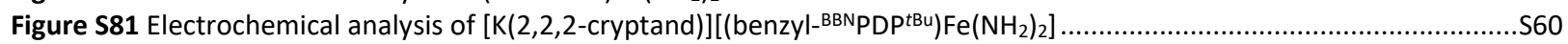

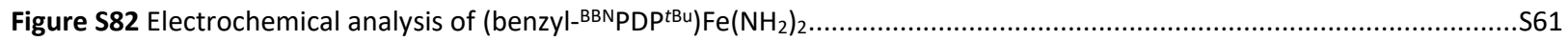

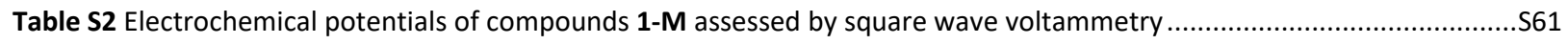

Table S3 Electrochemical potentials of compounds 2-M assessed by square wave voltammetry ..........................................561

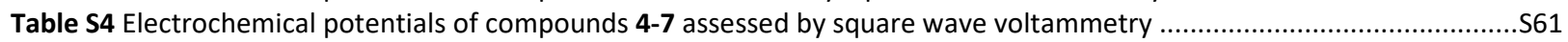

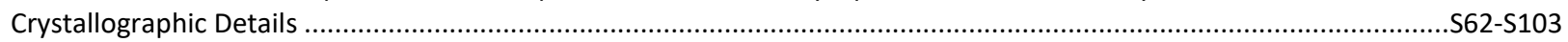

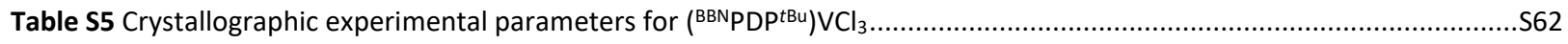

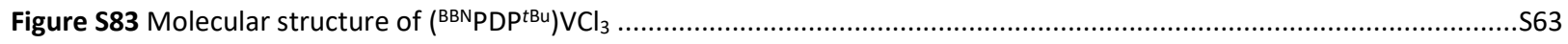

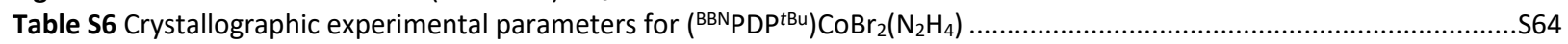

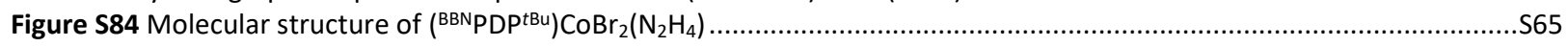

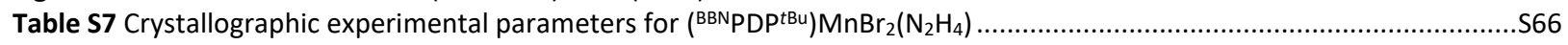

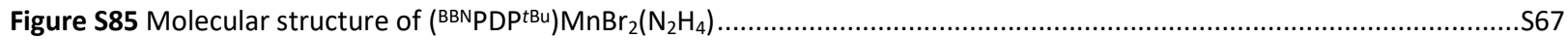

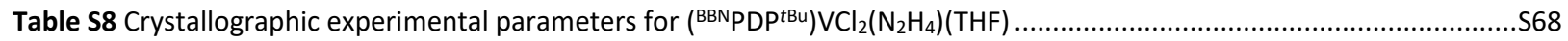

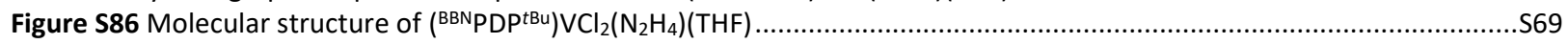

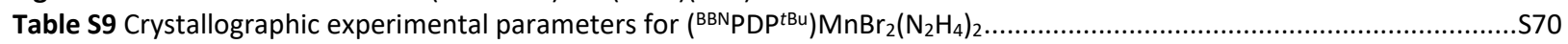

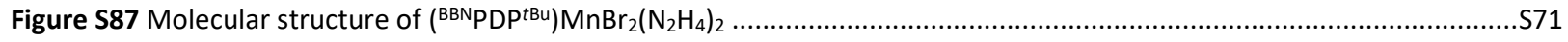

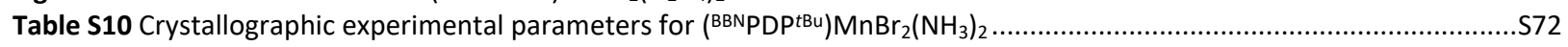

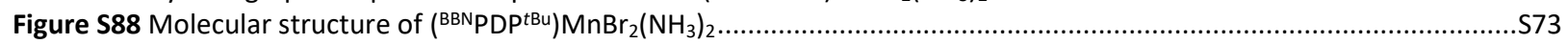

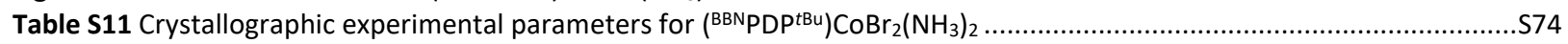

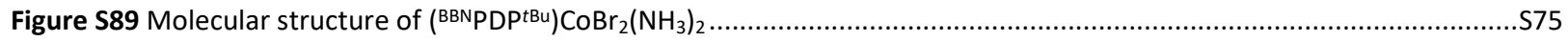

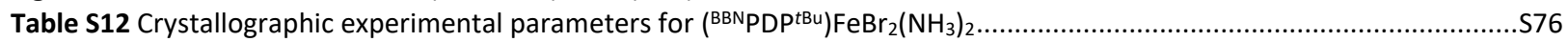

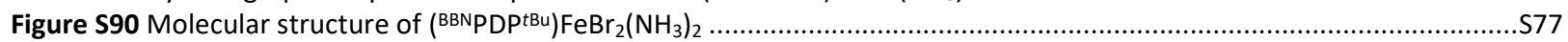

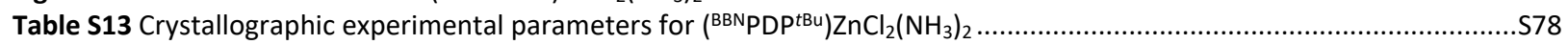

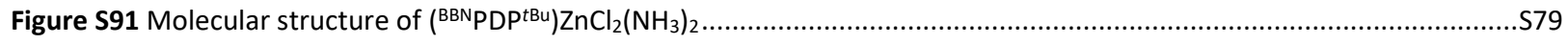

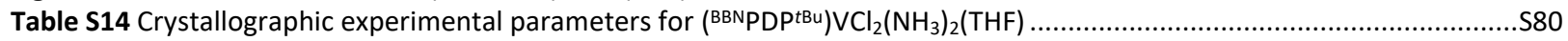

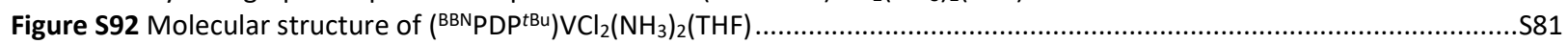

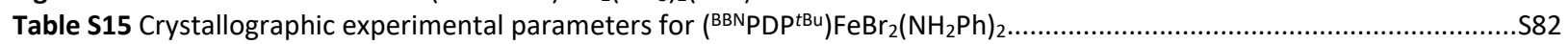

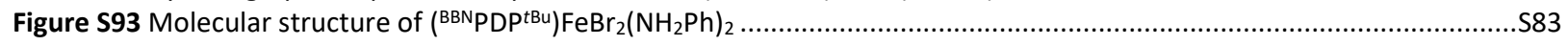

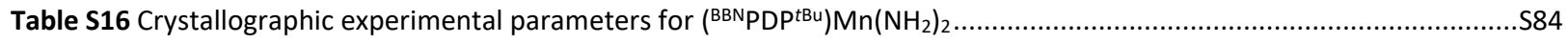

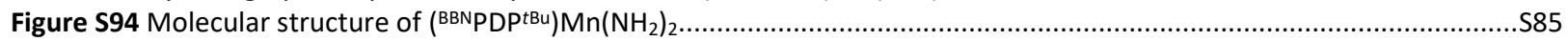

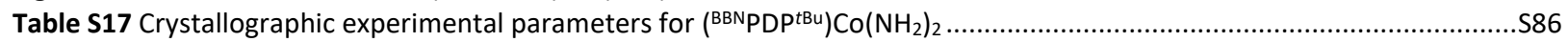

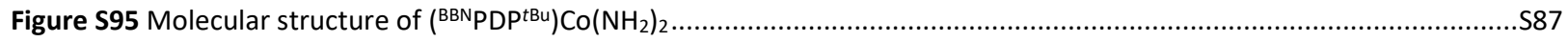




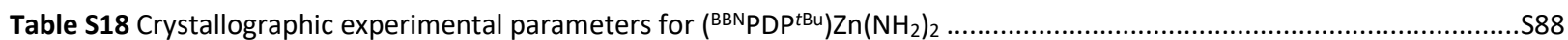

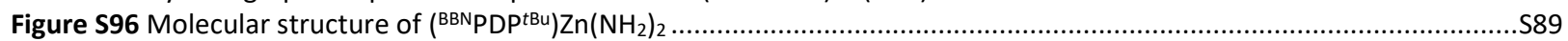

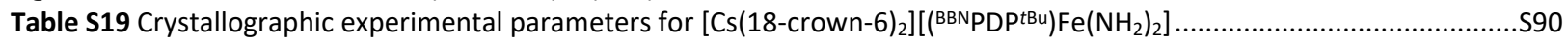

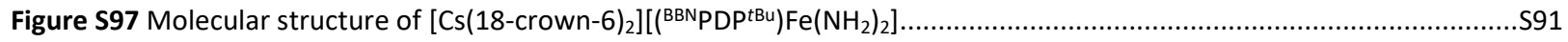

Table S20 Crystallographic experimental parameters for [K(2,2,2-cryptand)][(benzyl-BBNPDPtBu)Fe $\left.\left(\mathrm{NH}_{2}\right)_{2}\right]$...........................S92

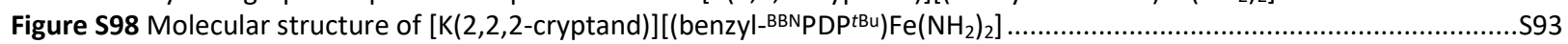

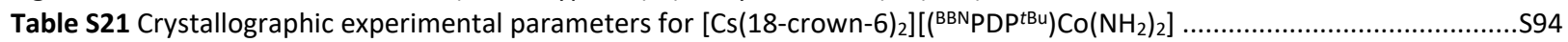

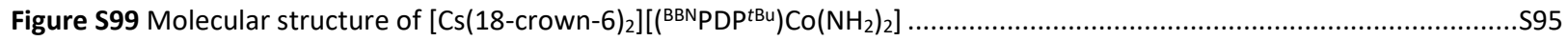

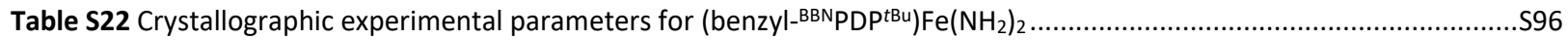

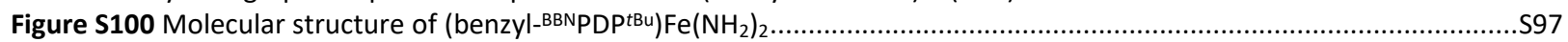

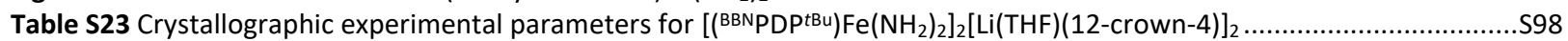

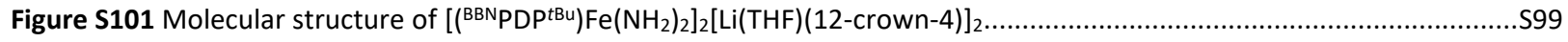

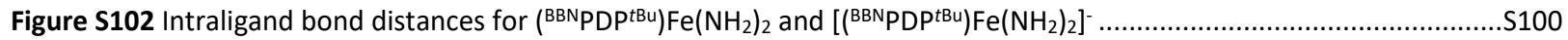

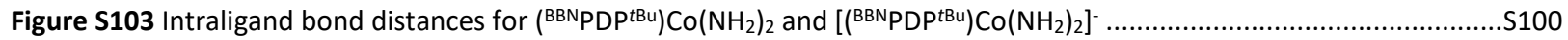

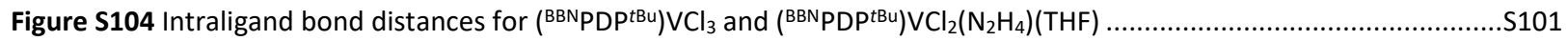

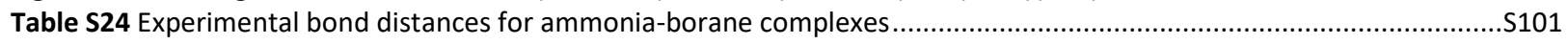

Table S25 Experimental bond distances for hydrazine captured divalent compounds ....................................................S102

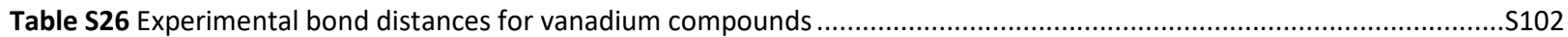

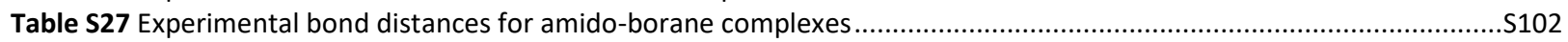

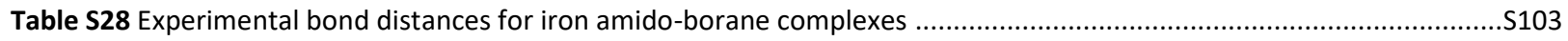

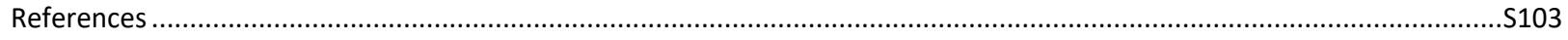


General Considerations. All air- and moisture-sensitive manipulations were performed using standard Schlenk techniques or in an inert atmosphere drybox with an atmosphere of purified nitrogen. The drybox was equipped with a cold well designed for freezing samples in liquid nitrogen as well as a $-35^{\circ} \mathrm{C}$ freezer for cooling samples and crystallizations. Solvents were purified using a Glass Contour solvent purification system through percolation through a $\mathrm{Cu}$ catalyst, molecular sieves, and alumina. Solvents were then stored over sodium and/or sieves. Benzene- $d_{6}$, THF- $d_{8}$, and chloroform- $d$ were purchased from Cambridge Isotope Laboratories. Benzene- $d_{6}$ was dried with molecular sieves and sodium, and degassed by three freeze-pump-thaw cycles. Chloroform- $d$ was distilled from $\mathrm{CaH}_{2}$. THF- $d_{8}$ was dried over molecular sieves. Anhydrous hydrazine was degassed by freeze-pump-thaw cycles and passed through alumina inside of a glovebox before use. Crown ethers, ammonia (0.4 M in THF), potassium bis(trimethylsilyl)amide, tetrabutylammonium chloride/bromide and metal dihalides were purchased from commercial vendors and used as received. $\mathrm{MC}_{8}{ }^{1}$ benzylpotassium, ${ }^{2}$ iodobenzene dichloride, ${ }^{3} \mathrm{AgBPh}_{4},{ }^{4}{ }^{\mathrm{BBN}} \mathrm{PDP}^{\mathrm{tBu}}$, $\left({ }^{\mathrm{BBN}} \mathrm{PDP}^{\mathrm{BB}}\right) \mathrm{FeBr}_{2}$, and $\left({ }^{\mathrm{BBN}} \mathrm{PDP}{ }^{\mathrm{BBu}}\right) \mathrm{ZnCl}_{2}{ }^{5}$ were synthesized according to literature procedures.

NMR spectra were recorded on Varian Vnmrs 700 or Varian MR400 spectrometers. ${ }^{1} \mathrm{H}$, ${ }^{13} \mathrm{C}$, and ${ }^{11} \mathrm{~B}$ chemical shifts are reported in parts per million $(\mathrm{ppm})$ relative to tetramethylsilane and referenced internally to the residual solvent peak. ${ }^{11} \mathrm{~B}$ spectra were referenced on a unified scale, where the single primary reference is the frequency of the residual solvent peak in the ${ }^{1} \mathrm{H}$ NMR spectrum. ${ }^{11} \mathrm{~B}$ spectra are referenced vs. $\mathrm{BF}_{3}\left(\mathrm{OEt}_{2}\right)$. Multiplicities are reported as follows: singlet $(\mathrm{s})$, doublet $(\mathrm{d})$, triplet $(\mathrm{t})$, quartet (q). Infrared spectra were recorded using a Nicolet iS10 FT-IR spectrometer. Samples were diluted into dry $\mathrm{KBr}$ and recorded as pellets. Electronic absorption spectra were recorded at ambient temperature in sealed $1 \mathrm{~cm}$ quartz cuvettes with a Varian Cary-50 spectrophotometer. Magnetic susceptibilities were obtained in solution by the Evans method. ${ }^{6}$ Details for individual complexes are listed below.

Single crystals of ( $\left.{ }^{\mathrm{BBN} P D P}{ }^{t B u}\right) \mathrm{VCl}_{3}$, ( $\left.{ }^{\text {BBN PDP }}{ }^{t B u}\right) \mathrm{CoBr}_{2}\left(\mathrm{~N}_{2} \mathrm{H}_{4}\right),\left({ }^{\text {BBN PDP }}{ }^{t B u}\right) \mathrm{MnBr}_{2}\left(\mathrm{~N}_{2} \mathrm{H}_{4}\right),\left({ }^{\text {BBN PDP }}{ }^{t B u}\right) \mathrm{ZnCl}_{2}\left(\mathrm{NH}_{3}\right)_{2}$, $\left({ }^{\text {BBNPDP }}{ }^{\mathrm{tBu}}\right) \mathrm{VCl}_{2}\left(\mathrm{NH}_{3}\right)_{2}(\mathrm{THF}), \quad\left({ }^{\mathrm{BBN} P D P}{ }^{\mathrm{BBu}}\right) \mathrm{VCl}_{2}\left(\mathrm{~N}_{2} \mathrm{H}_{4}\right) \quad(\mathrm{THF}), \quad\left({ }^{\mathrm{BBN} P D P}{ }^{\mathrm{tBu}}\right) \mathrm{Zn}\left(\mathrm{NH}_{2}\right)_{2}, \quad[\mathrm{Cs}(18-\mathrm{crown}-$ $\left.\left.6)_{2}\right]\left({ }^{\mathrm{BBN} P D P}{ }^{t B u}\right) \mathrm{Co}\left(\mathrm{NH}_{2}\right)_{2}\right]$, and $\left.\left[\mathrm{Cs}(18-\mathrm{crown}-6)_{2}\right]\left({ }^{\mathrm{BBN}} \mathrm{PDP}{ }^{\mathrm{tBu}}\right) \mathrm{Fe}\left(\mathrm{NH}_{2}\right)_{2}\right]$ suitable for X-ray diffraction were coated with poly(isobutylene) oil and quickly transferred to the goniometer head of a Bruker AXS D8 Quest diffractometer with kappa geometry, an I- $\mu$-S microsource X-ray tube, laterally graded multilayer (Goebel) mirror for monochromatization, a Photon2 CMOS area detector and an Oxford Cryosystems low temperature device. Examination and data collection were performed with $\mathrm{Cu}$ Ka radiation $(\lambda=1.54184$ $\AA$ ). Single crystals of $\left[\mathrm{K}\left(2,2,2\right.\right.$-cryptand)](benzyl- $\left.\left.{ }^{\mathrm{BBN} P D P}{ }^{\mathrm{tBu}}\right) \mathrm{Fe}\left(\mathrm{NH}_{2}\right)_{2}\right], \quad\left({ }^{\mathrm{BBN} P D P^{\mathrm{BB}}}\right) \mathrm{Co}\left(\mathrm{NH}_{2}\right)_{2}$,

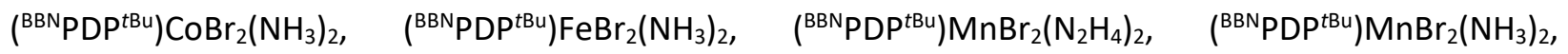
$\left({ }^{\text {BBNPDP }}{ }^{t B u}\right) \mathrm{Mn}\left(\mathrm{NH}_{2}\right)_{2}$, (benzyl- $\left.{ }^{\mathrm{BBN} P D P}{ }^{t B u}\right) \mathrm{Fe}\left(\mathrm{NH}_{2}\right)_{2}$, and [( $\left.\left.{ }^{\mathrm{BBN} P D P}{ }^{t B u}\right) \mathrm{Fe}\left(\mathrm{NH}_{2}\right)_{2}\right]_{2}\left[\mathrm{Li}(\mathrm{THF})(12 \text {-crown-4) }]_{2}\right.$ suitable for X-ray diffraction were coated with poly(isobutylene) oil and quickly transferred to the goniometer head of a Bruker AXS D8 Quest diffractometer with a fixed chi angle, a sealed tube fine focus X-ray tube, single crystal curved graphite incident beam monochromator and a Photon100 CMOS area detector. Examination and data collection were performed with Mo K $\alpha$ radiation $(\lambda=0.71073 \AA$ ). Single crystals of $\left({ }^{\mathrm{BBN}} \mathrm{PDP}{ }^{\mathrm{tBu}}\right) \mathrm{FeBr}_{2}\left(\mathrm{NH}_{2} \mathrm{Ph}\right)_{2}$ suitable for $\mathrm{X}$-ray diffraction were coated with poly(isobutylene) oil in a drybox and mounted on a Rigaku AFC10K Saturn 944+ CCD-based X-ray diffractometer equipped with a low temperature device and Micromax-007HF Cu-target microfocus rotating anode $(\lambda=1.54187 \AA)$. The data were collected using CrystalClear 2.011 and processed using CrysAlis PRO 1.171.38.41. Empirical absorption correction was applied using spherical harmonics, as implemented in the SCALE3 ABSPACK scaling algorithm. For both Quest instruments, data were collected, reflections were indexed and processed, and the files scaled and corrected for absorption using APEX3. ${ }^{7}$ For all samples, the space 
groups were assigned and the structures were solved by direct methods using XPREP within the SHELXTL suite of programs ${ }^{8}$ and refined by full matrix least squares against $F^{2}$ with all reflections using Shelx|2017 or Shelx $12018^{9}$ using the graphical interface Shelxle. ${ }^{10}$ If not specified otherwise, $\mathrm{H}$ atoms attached to carbon atoms were positioned geometrically and constrained to ride on their parent atoms, with carbon hydrogen bond distances of $0.95 \AA$ for aromatic $\mathrm{C}-\mathrm{H}, 1.00,0.99$ and $0.98 \AA$ for aliphatic $\mathrm{C}-\mathrm{H}, \mathrm{CH}_{2}$, and $\mathrm{CH}_{3}$ moieties, respectively. Depending on data quality, $\mathrm{N}-\mathrm{H}$ bond distances for pyramidal ( $\mathrm{sp}^{3}$ hybridized) ammonium $\mathrm{NH}_{2}{ }^{+}$and $\mathrm{NH}_{3}{ }^{+}$groups were either constrained to $0.91 \AA$, or refined and restrained to $0.91(2)$ $\AA$. $\mathrm{H}$ atoms of pyramidalized $\mathrm{R}_{2} \mathrm{NH}$ and $\mathrm{RNH}_{2}$ units were refined and $\mathrm{N}-\mathrm{H}$ distances were restrained to $0.88(2) \AA$. Methyl and $\mathrm{NH}_{3}{ }^{+} \mathrm{H}$ atoms were allowed to rotate but not to tip to best fit the experimental electron density. $\mathrm{U}_{\text {iso }}(\mathrm{H})$ values were set to a multiple of $\mathrm{U}_{\text {eq }}(\mathrm{C})$ with 1.5 for $\mathrm{CH}_{3}$ and $\mathrm{NH}_{3}{ }^{+}$, and 1.2 for $\mathrm{NH}_{2}$, $\mathrm{NH}_{2}{ }^{+}, \mathrm{CH}_{2}$, and $\mathrm{C}-\mathrm{H}$ units, respectively. Additional data collection and refinement details, including description of disorder or twinning (where present) can be found with the individual structure descriptions, below. CCDC 1993625-1993643.

Synthesis of ( $\left.{ }^{\text {BBN PDP }}{ }^{\mathrm{tBu}}\right) \mathrm{MnBr}_{2}$. A $20 \mathrm{~mL}$ scintillation vial was charged with $\mathrm{MnBr}_{2}$ (0.047 g, $\left.0.219 \mathrm{mmol}\right)$, ${ }^{B B N} P_{D P} P^{t B u}(0.140 \mathrm{~g}, 0.216 \mathrm{mmol})$, and $8 \mathrm{~mL} \mathrm{DCM}$ and stirred $18 \mathrm{hr}$ resulting in a colorless solution. The solution was filtered through Celite, dried, and washed with $2 \times 10 \mathrm{~mL} n$-pentane to afford off-white powder (0.173 g, $0.201 \mathrm{mmol}, 93 \%$ ) assigned as ( $\left.{ }^{\mathrm{BBN} P D P}{ }^{\mathrm{tBu}}\right) \mathrm{MnBr}_{2}$. MALDI-TOF of $\mathrm{C}_{41} \mathrm{H}_{63} \mathrm{~N}_{5} \mathrm{~B}_{2} \mathrm{Br}_{2} \mathrm{Mn}_{1}-\mathrm{Br}$ : Calc. 781.383; Found 781.236. ${ }^{1} \mathrm{H} \mathrm{NMR}\left(\mathrm{CDCl}_{3}, 25^{\circ} \mathrm{C}\right) \delta=-27.89$ (2500), 1.38 (100), 1.68 (145), 1.93 (110), 2.05 (190), 3.70 (550), 36.05 (2300), 56.76 (3300), 88.19 (4700). Selected IR data (KBr) $v=3118,1610$, $1575,1455,1018,795 \mathrm{~cm}^{-1}$. UV-Vis (THF, ambient temperature; $\lambda_{\max }$, molar absorptivity): $332 \mathrm{~nm}, 8500$ $\mathrm{M}^{-1} \mathrm{~cm}^{-1} ; 266 \mathrm{~nm}, 16700 \mathrm{M}^{-1} \mathrm{~cm}^{-1} . \mu_{\text {eff }}=5.79+/-0.05 \mu_{\mathrm{B}}\left(25^{\circ} \mathrm{C}, \mathrm{THF}\right)$.

Synthesis of ( $\left.{ }^{\mathrm{BBN} P D P}{ }^{t \mathrm{Bu}}\right) \mathrm{CoBr}_{2}$. A $20 \mathrm{~mL}$ scintillation vial was charged with $\mathrm{CoBr}_{2}(0.168 \mathrm{~g}, 0.768 \mathrm{mmol})$, ${ }^{B B N} P_{D P}^{t B u}(0.500 \mathrm{~g}, 0.772 \mathrm{mmol})$, and $20 \mathrm{~mL} \mathrm{DCM}$ and stirred $36 \mathrm{hr}$ resulting in a green solution. The solution was filtered through Celite, dried, and washed with $2 \times 10 \mathrm{~mL} n$-pentane to afford light green powder (0.542 g, $0.626 \mathrm{mmol}, 81 \%$ ) assigned as ( $\left.{ }^{\mathrm{BBN} P D P}{ }^{\mathrm{BBu}}\right) \mathrm{CoBr}_{2}$. MALDI-TOF of $\mathrm{C}_{41} \mathrm{H}_{63} \mathrm{~N}_{5} \mathrm{~B}_{2} \mathrm{Br}_{2} \mathrm{CO}_{1}-\mathrm{Br}$ : Calc. 785.378; Found 785.279. ${ }^{1} \mathrm{H}$ NMR $\left(\mathrm{CDCl}_{3}, 25^{\circ} \mathrm{C}\right) \delta=-44.80\left(109,4 \mathrm{H}, \mathrm{CH}_{2}\right),-43.34\left(156,4 \mathrm{H}, \mathrm{CH}_{2}\right)$, $11.60\left(11,18 \mathrm{H}, \mathrm{C}\left(\mathrm{CH}_{3}\right)_{3}\right),-6.34(29,4 \mathrm{H}),-3.23(29,1 \mathrm{H}, p$-pyridine- $\mathrm{CH}),-1.38(20,4 \mathrm{H}), 1.98(35,8 \mathrm{H}, \mathrm{BBN}-$ $\mathrm{CH}), 2.04(8 \mathrm{H}$ and $4 \mathrm{H}$ overlapping, $\mathrm{BBN}-\mathrm{CH}), 2.30(30,4 \mathrm{H}), 23.02(21,2 \mathrm{H}$, pyridine- or pyrazole- $\mathrm{CH}), 69.11$ $\left(32,2 \mathrm{H}\right.$, pyridine- or pyrazole-CH). Selected IR data $(\mathrm{KBr}) \mathrm{v}=3119,1612,1574,1455,816,795 \mathrm{~cm}^{-1}$. UVVis (THF, ambient temperature; $\lambda_{\max }$, molar absorptivity): $334 \mathrm{~nm}, 10300 \mathrm{M}^{-1} \mathrm{~cm}^{-1}$. $\mu_{\text {eff }}=4.20+/-0.05 \mu_{\mathrm{B}}$ $\left(25{ }^{\circ} \mathrm{C}, \mathrm{CDCl}_{3}\right)$.

Synthesis of ( $\left.{ }^{B B N P D P}{ }^{\mathrm{tBu}}\right) \mathrm{VCl}_{3}$. A $20 \mathrm{~mL}$ scintillation vial was charged with $\mathrm{VCl}_{3}(0.121 \mathrm{~g}, 0.769 \mathrm{mmol})$, ${ }_{\text {BBNPDP }}^{\text {tBu }}(0.500 \mathrm{~g}, 0.772 \mathrm{mmol})$, and $20 \mathrm{~mL} \mathrm{DCM}$ and stirred $36 \mathrm{hr}$ resulting in a yellow-green solution. The solution was filtered through Celite, dried, and washed with $2 \times 10 \mathrm{~mL} n$-pentane to afford yellow powder $(0.447 \mathrm{~g}, 0.555 \mathrm{mmol}, 72 \%)$ assigned as $\left({ }^{B B N} \mathrm{PDP}^{\mathrm{B} \mathrm{u}}\right) \mathrm{VCl}_{3}$. Single, $\mathrm{X}$-ray quality crystals were obtained by diffusing diethyl ether into a dichloromethane solution of $\left({ }^{B B N} \mathrm{PDP}^{\mathrm{tBu}}\right) \mathrm{VCl}_{3}$ at room temperature. MALDI-TOF of $\mathrm{C}_{41} \mathrm{H}_{63} \mathrm{~N}_{5} \mathrm{~B}_{2} \mathrm{Cl}_{3} \mathrm{~V}_{1}$ - Cl: Calc. 768.409; Found 767.951. ${ }^{1} \mathrm{H} \mathrm{NMR}\left(\mathrm{C}_{6} \mathrm{D}_{6}, 25{ }^{\circ} \mathrm{C}\right) \delta=$ -1.99 (14, 4H), $0.87(21,2 \mathrm{H}), 1.18(28,2 \mathrm{H}, \mathrm{Ar}-\mathrm{CH}), 1.41\left(6,18 \mathrm{H}, \mathrm{C}\left(\mathrm{CH}_{3}\right)_{3}\right), 1.64(26,8 \mathrm{H}, \mathrm{BBN}-\mathrm{CH}), 1.67(20$, 4H), $1.80(12,4 \mathrm{H}), 1.82$ (13, 8H, BBN-CH), 3.45 (24, 2H, Ar- $\mathrm{CH}), 3.99(800,4 \mathrm{H}), 4.06$ (19, 1H, p-pyr-CH), $7.97(620,4 \mathrm{H})$. Selected IR data $(\mathrm{KBr}) \mathrm{v}=3127,1613,1577,1493,1459,1367,1278,1153,795 \mathrm{~cm}^{-1}$. UVVis (THF, ambient temperature; $\lambda_{\text {max }}$ molar absorptivity): $320 \mathrm{~nm}, 10700 \mathrm{M}^{-1} \mathrm{~cm}^{-1} \cdot \mu_{\text {eff }}=2.7+/-0.1 \mu_{\mathrm{B}}(25$ $\left.{ }^{\circ} \mathrm{C}, \mathrm{CDCl}_{3}\right)$. 


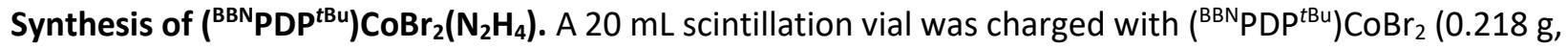
$0.252 \mathrm{mmol}$ ) and $8 \mathrm{~mL}$ THF. Hydrazine $(0.0079 \mathrm{~mL}, 0.252 \mathrm{mmol})$ was dissolved in $1 \mathrm{~mL}$ THF and added dropwise to a stirring solution containing $\left({ }^{\mathrm{BBN}} \mathrm{PDP}^{\mathrm{B} u}\right) \mathrm{CoBr}_{2}$. After $10 \mathrm{~min}$, volatiles were removed in vacuo. The material was washed with $20 \mathrm{~mL} n$-pentane and dried to afford light green powder $(0.213 \mathrm{~g}, 0.237$ mmol, 94\%) assigned as ( $\left.{ }^{B B N} P D P^{t B u}\right) \mathrm{CoBr}_{2}\left(\mathrm{~N}_{2} \mathrm{H}_{4}\right)$. Single, X-ray quality crystals were obtained by diffusing $n$ pentane into a dichloromethane solution of ( $\left.{ }^{\mathrm{BBN} P D P}{ }^{\mathrm{BBu}}\right) \mathrm{CoBr}_{2}\left(\mathrm{~N}_{2} \mathrm{H}_{4}\right)$ at room temperature. MALDI-TOF of $\mathrm{C}_{41} \mathrm{H}_{67} \mathrm{~N}_{7} \mathrm{~B}_{2} \mathrm{Br}_{2} \mathrm{CO}_{1}-\left(\mathrm{Br}+\mathrm{N}_{2} \mathrm{H}_{4}\right)$ : Calc. 785.378; Found 784.796. ${ }^{1} \mathrm{H} \mathrm{NMR}\left(\mathrm{CDCl}_{3}, 2{ }^{\circ} \mathrm{C}\right) \delta=-36.59(530,4 \mathrm{H}$, $\left.\mathrm{CH}_{2}\right),-27.90\left(380,4 \mathrm{H}, \mathrm{CH}_{2}\right),-9.03\left(9,18 \mathrm{H}, \mathrm{C}\left(\mathrm{CH}_{3}\right)_{3}\right),-5.27(23,1 \mathrm{H}, p$-pyr- $\mathrm{CH}), 2.46(57,4 \mathrm{H}), 3.17(46,8 \mathrm{H}$, overlapping peaks), $3.50\left(61,8 \mathrm{H}\right.$, overlapping peaks), $4.89(81,4 \mathrm{H}), 6.13(100,4 \mathrm{H}), 21.54\left(520,4 \mathrm{H}, \mathrm{N}_{2} \mathrm{H}_{4}\right)$, $26.58(22,2 \mathrm{H}$, pyridine- or pyrazole- $\mathrm{CH}), 72.51(32,2 \mathrm{H}$, pyridine- or pyrazole- $\mathrm{CH})$. A CH $\mathrm{CH}_{2}$ resonance $(4 \mathrm{H})$ is not observed. Selected IR data $(\mathrm{KBr}) \mathrm{v}=3283(\mathrm{~N}-\mathrm{H}), 3208(\mathrm{~N}-\mathrm{H}), 3162(\mathrm{~N}-\mathrm{H}), 3101(\mathrm{~N}-\mathrm{H}), 1611,1575,1491$, $1456,1246,897,791 \mathrm{~cm}^{-1}$. The poor solubility of this compound precluded accurate solution magnetic susceptibility measurements. UV-Vis (THF, ambient temperature; $\lambda_{\max }$, molar absorptivity): $340 \mathrm{~nm}, 8200$ $\mathrm{M}^{-1} \mathrm{~cm}^{-1}$.

Synthesis of ( $\left.{ }^{\text {BBN PDP }}{ }^{\mathrm{BBu}}\right) \mathrm{MnBr}_{2}\left(\mathrm{~N}_{2} \mathrm{H}_{4}\right)$. A $20 \mathrm{~mL}$ scintillation vial was charged with ( $\left.{ }^{\mathrm{BBN} P D P} \mathrm{PDu}^{\mathrm{tBu}}\right) \mathrm{MnBr}_{2}(0.253$ $\mathrm{g}, 0.293 \mathrm{mmol}$ ) and $8 \mathrm{~mL}$ THF. Hydrazine $(0.0092 \mathrm{~mL}, 0.293 \mathrm{mmol})$ was dissolved in $1 \mathrm{~mL}$ THF and added dropwise to the stirring solution containing ( $\left.{ }^{B B N} \mathrm{PDP}^{\mathrm{tBu}}\right) \mathrm{MnBr}_{2}$. After $10 \mathrm{~min}$, volatiles were removed in vacuo. The material was washed with $20 \mathrm{~mL} n$-pentane and dried to afford off-white powder $(0.251 \mathrm{~g}$, $0.281 \mathrm{mmol}, 96 \%)$ assigned as ( $\left.{ }^{\mathrm{BBN}} \mathrm{PDP}{ }^{\mathrm{BB} u}\right) \mathrm{MnBr}_{2}\left(\mathrm{~N}_{2} \mathrm{H}_{4}\right)$. Single, $\mathrm{X}$-ray quality crystals were obtained by layering a dichloromethane solution of ( $\left.{ }^{\mathrm{BBN} P D P}{ }^{\mathrm{BB}}\right) \mathrm{MnBr}_{2}\left(\mathrm{~N}_{2} \mathrm{H}_{4}\right)$ with $n$-pentane at room temperature. An additional equivalent of hydrazine was found to produce ( $\left.{ }^{\mathrm{BBN} P D P}{ }^{\mathrm{BBu}}\right) \mathrm{MnBr}_{2}\left(\mathrm{~N}_{2} \mathrm{H}_{4}\right)_{2}$ as determined by X-ray crystallography (see structure below; obtained by layering a DCM solution with $n$-pentane at room temperature) and IR spectroscopy. MALDI-TOF of $\mathrm{C}_{41} \mathrm{H}_{67} \mathrm{~N}_{7} \mathrm{~B}_{2} \mathrm{Br}_{2} \mathrm{Mn}_{1}-\left(\mathrm{Br}+\mathrm{N}_{2} \mathrm{H}_{4}\right)$ : Calc. 781.383; Found 780.811. ${ }^{1} \mathrm{H}$ NMR (THF, $25{ }^{\circ} \mathrm{C}$ ) resonances are not observed for this compound. Selected IR data $(\mathrm{KBr}) \mathrm{v}=$ $3283(\mathrm{~N}-\mathrm{H}), 3209(\mathrm{~N}-\mathrm{H}), 3162(\mathrm{~N}-\mathrm{H}), 3096(\mathrm{~N}-\mathrm{H}), 1608,1575,1367,897,792 \mathrm{~cm}^{-1}$. The poor solubility of this compound precluded accurate solution magnetic susceptibility measurements. UV-Vis (THF, ambient temperature; $\lambda_{\max }$, molar absorptivity): $333 \mathrm{~nm}, 11000 \mathrm{M}^{-1} \mathrm{~cm}^{-1}$.

Synthesis of ( $\left.{ }^{\mathrm{BBN}} \mathrm{PDP}{ }^{\mathrm{BBu}}\right) \mathrm{MnBr}_{2}\left(\mathrm{NH}_{3}\right)_{2}$. A $20 \mathrm{~mL}$ scintillation vial was charged with ( $\left.{ }^{\mathrm{BBN} P D P} \mathrm{PBu}^{\mathrm{B} u}\right) \mathrm{MnBr}_{2}(0.319$ $\mathrm{g}, 0.370 \mathrm{mmol}$ ) and $6 \mathrm{~mL}$ THF. While stirring, ammonia $(0.4 \mathrm{M}$ in THF, $1.860 \mathrm{mmol})$ was added. The colorless solution was stirred $15 \mathrm{~min}$ and volatiles were removed in vacuo. The solid was washed with $2 \mathrm{x}$ $10 \mathrm{~mL} n$-pentane and dried to afford off-white powder $(0.306 \mathrm{~g}, 0.341 \mathrm{mmol}, 92 \%)$ assigned as ( $\left.{ }^{\mathrm{BBN} P D P}{ }^{\mathrm{tBu}}\right) \mathrm{MnBr}_{2}\left(\mathrm{NH}_{3}\right)_{2}$. Single, $\mathrm{X}$-ray quality crystals were obtained from slow diffusion of $n$-pentane into a DCM solution at room temperature. MALDI-TOF of $\mathrm{C}_{41} \mathrm{H}_{69} \mathrm{~N}_{7} \mathrm{~B}_{2} \mathrm{Br}_{2} \mathrm{Mn}_{1}-\left(\mathrm{Br}+2 \mathrm{NH}_{3}\right)$ : Calc. 781.383; Found 781.254. ${ }^{1} \mathrm{H}$ NMR $\left(\mathrm{CDCl}_{3}, 25{ }^{\circ} \mathrm{C}\right) \delta=-27.88$ (1900), 1.61 (260), 3.54 (400), 34.36 (1900), 56.32 (2600). Selected IR data $(\mathrm{KBr}) \mathrm{v}=3357(\mathrm{~N}-\mathrm{H}), 3312(\mathrm{~N}-\mathrm{H}), 3238(\mathrm{~N}-\mathrm{H}), 3176(\mathrm{~N}-\mathrm{H}), 1610,1575,1456,817,795 \mathrm{~cm}^{-}$ 1. UV-Vis (THF, ambient temperature; $\lambda_{\max }$, molar absorptivity): $334 \mathrm{~nm}, 11400 \mathrm{M}^{-1} \mathrm{~cm}^{-1} ; 268 \mathrm{~nm}, 21400 \mathrm{M}^{-}$ ${ }^{1} \mathrm{~cm}^{-1} \cdot \mu_{\text {eff }}=5.88+/-0.10 \mu_{\mathrm{B}}\left(25^{\circ} \mathrm{C}, \mathrm{THF}\right)$. 
Synthesis of ( $\left.{ }^{\mathrm{BBN} P D P} \mathrm{P}^{\mathrm{tBu}}\right) \mathrm{FeBr}_{2}\left(\mathrm{NH}_{3}\right)_{2}$. A $100 \mathrm{~mL}$ round bottom flask was charged with $\left({ }^{\mathrm{BBN} P D P}{ }^{\mathrm{tBu}}\right) \mathrm{FeBr}_{2}$ $(2.075 \mathrm{~g}, 2.404 \mathrm{mmol})$ and $15 \mathrm{~mL} \mathrm{DCM}$. While stirring, ammonia $(0.4 \mathrm{M}$ in THF, $12.000 \mathrm{mmol}$ ) was added. After $15 \mathrm{~min}$, volatiles were removed in vacuo to afford light orange powder assigned as $\left({ }^{B B N} \mathrm{PDP}^{\mathrm{ABu}}\right) \mathrm{FeBr}_{2}\left(\mathrm{NH}_{3}\right)_{2}$ in quantitative yield. Single, X-ray quality crystals were obtained from slow diffusion of $n$-pentane into a DCM solution at room temperature. MALDI-TOF of $\mathrm{C}_{41} \mathrm{H}_{69} \mathrm{~N}_{7} \mathrm{~B}_{2} \mathrm{Br}_{2} \mathrm{Fe}_{1}-2 \mathrm{NH}_{3}$ : Calc. 863.248; Found 863.346. ${ }^{1} \mathrm{H}$ NMR $\left(\mathrm{C}_{6} \mathrm{D}_{6}, 25{ }^{\circ} \mathrm{C}\right) \delta=-32.70(56,1 \mathrm{H}, p$-pyridine- $\mathrm{CH}),-16.31(73,6 \mathrm{H}$, $\left.\mathrm{NH}_{3}\right),-4.20(26,4 \mathrm{H}),-3.71(42,4 \mathrm{H}),-1.07(37,2 \mathrm{H}, \mathrm{BBN}-\mathrm{CH}),-0.87(34,4 \mathrm{H}), 0.10(42,2 \mathrm{H}, \mathrm{BBN}-\mathrm{CH}), 0.83(30$, 4H), 1.09 (52, 2H, BB-CH), $1.39(18,4 \mathrm{H}), 1.74$ (59, 2H and 4H overlapping), $3.48(20,4 \mathrm{H}), 10.47(29,18 \mathrm{H}$, $\left.\mathrm{C}\left(\mathrm{CH}_{3}\right)_{3}\right), 37.20\left(\mathrm{~N}-\mathrm{CH}_{2}\right), 45.03(66,2 \mathrm{H}$, pyridine- or pyrazole- $\mathrm{CH}), 50.45(52,2 \mathrm{H}$, pyridine- or pyrazole- $\mathrm{CH})$. Selected IR data (KBr) v = $3354(\mathrm{~N}-\mathrm{H}), 3314(\mathrm{~N}-\mathrm{H}), 3239(\mathrm{~N}-\mathrm{H}), 3179(\mathrm{~N}-\mathrm{H}), 1610,1575,794,739 \mathrm{~cm}^{-1}$. UVVis (THF, ambient temperature; $\lambda_{\max }$, molar absorptivity): $338 \mathrm{~nm}, 9400 \mathrm{M}^{-1} \mathrm{~cm}^{-1}$. $\mu_{\text {eff }}=5.27+/-0.10 \mu_{\mathrm{B}}(25$ $\left.{ }^{\circ} \mathrm{C}, \mathrm{THF}\right)$.

Synthesis of ( $\left.{ }^{\mathrm{BBN} P D P}{ }^{\mathrm{BBu}}\right) \mathrm{CoBr}_{2}\left(\mathrm{NH}_{3}\right)_{2}$. A $20 \mathrm{~mL}$ scintillation vial was charged with ( $\left.{ }^{\mathrm{BBN} P D P}{ }^{\mathrm{BBu}}\right) \mathrm{CoBr}_{2}(0.300 \mathrm{~g}$, $0.346 \mathrm{mmol}$ ) and $8 \mathrm{~mL}$ THF. While stirring, ammonia (0.4 M in THF, $1.780 \mathrm{mmol}$ ) was added. The green solution was stirred $15 \mathrm{~min}$ and volatiles were removed in vacuo. The solid was washed with $2 \times 10 \mathrm{~mL} n$ pentane and dried to afford light green powder $(0.293 \mathrm{~g}, 0.325 \mathrm{mmol}, 94 \%)$ assigned as $\left({ }^{\mathrm{BBN} P D P}{ }^{\mathrm{BB} u}\right) \mathrm{CoBr}_{2}\left(\mathrm{NH}_{3}\right)_{2}$. Single, $\mathrm{X}$-ray quality crystals were obtained from slow diffusion of $n$-pentane into a DCM solution at room temperature. MALDI-TOF of $\mathrm{C}_{41} \mathrm{H}_{69} \mathrm{~N}_{7} \mathrm{~B}_{2} \mathrm{Br}_{2} \mathrm{Co}_{1}-\left(\mathrm{Br}+2 \mathrm{NH}_{3}\right)$ : Calc. 785.378; Found 785.270. ${ }^{1} \mathrm{H}$ NMR $\left(\mathrm{CDCl}_{3}, 25^{\circ} \mathrm{C}\right) \delta=-46.22\left(140,4 \mathrm{H}, \mathrm{CH}_{2}\right),-33.76\left(173,4 \mathrm{H}, \mathrm{CH}_{2}\right),-10.91\left(39,18 \mathrm{H}, \mathrm{C}\left(\mathrm{CH}_{3}\right)_{3}\right)$, $-2.54(52,4 \mathrm{H}),-1.83(40,4 \mathrm{H}),-1.54(58,4 \mathrm{H}),-0.89(50,2 \mathrm{H}, \mathrm{BBN}-\mathrm{CH}),-0.27(54,4 \mathrm{H}), 0.31(61,2 \mathrm{H}, \mathrm{BBN}-\mathrm{CH})$, $1.66(64,4 \mathrm{H}), 2.10(55,2 \mathrm{H}, \mathrm{BBN}-\mathrm{CH}), 2.84(57,4 \mathrm{H}), 3.75(42,1 \mathrm{H}, p$-pyridine- $\mathrm{CH}), 3.99(47,2 \mathrm{H}, \mathrm{BBN}-\mathrm{CH})$, $9.48\left(76,6 \mathrm{H}, \mathrm{NH}_{3}\right), 26.76(47,2 \mathrm{H}$, pyridine- or pyrazole- $\mathrm{CH}), 77.91(65,2 \mathrm{H}$, pyridine- or pyrazole- $\mathrm{CH})$. Selected IR data $(\mathrm{KBr}) \mathrm{v}=3350(\mathrm{~N}-\mathrm{H}), 3310(\mathrm{~N}-\mathrm{H}), 3241(\mathrm{~N}-\mathrm{H}), 3176(\mathrm{~N}-\mathrm{H}), 1612,1575,1492,1456,792$ $\mathrm{cm}^{-1}$. UV-Vis (THF, ambient temperature; $\lambda_{\max }$, molar absorptivity): $339 \mathrm{~nm}, 8300 \mathrm{M}^{-1} \mathrm{~cm}^{-1}$. $\mu_{\text {eff }}=4.8+/-0.1$ $\mu_{\mathrm{B}}\left(25^{\circ} \mathrm{C}, \mathrm{THF}\right)$.

Synthesis of ( $\left.{ }^{\mathrm{BBN} P D P}{ }^{\mathrm{BBu}}\right) \mathrm{ZnCl}_{2}\left(\mathrm{NH}_{3}\right)_{2}$. A $20 \mathrm{~mL}$ scintillation vial was charged with ( $\left.{ }^{\mathrm{BBN} P D P^{t B u}}\right) \mathrm{ZnCl}_{2}(0.670 \mathrm{~g}$, $0.855 \mathrm{mmol}$ ) and $9 \mathrm{~mL}$ THF. While stirring, ammonia (0.4 M in THF, $4.400 \mathrm{mmol}$ ) was added. The colorless solution was stirred $15 \mathrm{~min}$ and volatiles were removed in vacuo. The solid was washed with $3 \times 10 \mathrm{~mL} n$ pentane and dried to afford colorless powder (0.691 $\mathrm{g}, 0.845 \mathrm{mmol}, 99 \%)$ assigned as $\left({ }^{\mathrm{BBN} P D P}{ }^{\mathrm{BB} u}\right) \mathrm{ZnCl}_{2}\left(\mathrm{NH}_{3}\right)_{2}$. Single, $\mathrm{X}$-ray quality crystals were obtained from slow diffusion of $n$-pentane into a DCM solution at room temperature. MALDI-TOF of $\mathrm{C}_{41} \mathrm{H}_{69} \mathrm{~N}_{7} \mathrm{~B}_{2} \mathrm{Cl}_{2} \mathrm{Zn}_{1}-\left(\mathrm{Cl}+2 \mathrm{NH}_{3}\right)$ : Calc. 746.425; Found 746.314. ${ }^{1} \mathrm{H}$ NMR $\left(\mathrm{CDCl}_{3}, 25^{\circ} \mathrm{C}\right) \delta=0.44-0.52\left(\mathrm{~m}, 8 \mathrm{H}, \mathrm{B}-\mathrm{CH}_{2} \& \mathrm{~B}-\mathrm{CH}\right), 1.43-1.52(\mathrm{~m}, 6 \mathrm{H}, \mathrm{BBN}-\mathrm{CH} \times 2), 1.46$ $\left(\mathrm{s}, 18 \mathrm{H}, \mathrm{C}\left(\mathrm{CH}_{3}\right)_{3}\right), 1.57-1.64(\mathrm{~m}, 4 \mathrm{H}, \mathrm{BBN}-\mathrm{CH}), 1.70-1.83\left(\mathrm{~m}, 14 \mathrm{H}, \mathrm{BBN}-\mathrm{CH}^{\prime} \mathrm{s}\right), 2.05\left(\mathrm{~m}, 4 \mathrm{H}, \mathrm{CH}_{2} \mathrm{CH}_{2} \mathrm{CH}_{2}\right), 3.53$ $\left(\mathrm{s}, 6 \mathrm{H}, \mathrm{NH}_{3}\right), 4.49\left(\mathrm{t}, \mathrm{J}=8.5,4 \mathrm{H}, \mathrm{N}-\mathrm{CH}_{2}\right), 6.49(\mathrm{~s}, 2 \mathrm{H}$, pyrazole- $\mathrm{CH}), 7.59(\mathrm{~d}, J=8.0,2 \mathrm{H}, \mathrm{m}$-pyridine- $\mathrm{CH}), 7.97$ $(\mathrm{t}, J=8.0,1 \mathrm{H}, p$-pyridine- $\mathrm{CH}) .{ }^{13} \mathrm{C} \mathrm{NMR}\left(\mathrm{CDCl}_{3}, 25^{\circ} \mathrm{C}\right) \delta=19.66\left(\mathrm{~B}-\mathrm{CH}_{2}\right), 24.61(\mathrm{~B}-\mathrm{CH}), 24.86,25.27,28.01$, $30.22\left(\mathrm{C}\left(\mathrm{CH}_{3}\right)_{3}\right), 31.49(\mathrm{BBN}-\mathrm{CH}), 32.10,32.23(\mathrm{BBN}-\mathrm{CH}), 55.70\left(\mathrm{~N}-\mathrm{CH}_{2}\right), 100.99$ (pyrazole- $\left.\mathrm{CH}\right), 118.89(\mathrm{~m}-$ pyridine- $\mathrm{CH}$ ), 141.42 (p-pyridine-CH), $143.25(\mathrm{Ar}-\mathrm{C}), 148.28(\mathrm{Ar}-\mathrm{C}), 155.23(\mathrm{Ar}-\mathrm{C}) .{ }^{11} \mathrm{~B} \mathrm{NMR}\left(\mathrm{CDCl}_{3}, 25^{\circ} \mathrm{C}\right) \delta$ $=-5.19\left(B-\mathrm{NH}_{3}\right)$. Selected IR data $(\mathrm{KBr}) \mathrm{v}=3352(\mathrm{~N}-\mathrm{H}), 3332(\mathrm{~N}-\mathrm{H}), 3285(\mathrm{~N}-\mathrm{H}), 3255(\mathrm{~N}-\mathrm{H}), 3170(\mathrm{~N}-\mathrm{H})$, $1612,1575,1455,1242,1048,796 \mathrm{~cm}^{-1}$. UV-Vis (THF, ambient temperature; $\lambda_{\max }$, molar absorptivity): 335 $\mathrm{nm}, 8200 \mathrm{M}^{-1} \mathrm{~cm}^{-1}$. 


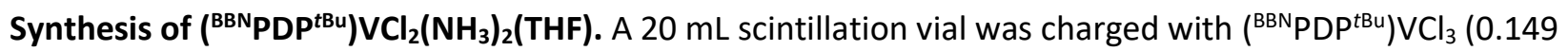
$\mathrm{g}, 0.185 \mathrm{mmol})$ and $8 \mathrm{~mL}$ THF and frozen. Upon thawing, potassium graphite $(0.025 \mathrm{~g}, 0.185 \mathrm{mmol})$ was added resulting in a rapid color change to deep blue. The reaction was stirred for $30 \mathrm{~min}$, filtered, and dried. The material was extracted into $4 \mathrm{~mL}$ toluene, filtered, and dried. The material was redissolved in $2 \mathrm{~mL}$ THF and ammonia ( $0.5 \mathrm{M}$ in dioxane, $1.300 \mathrm{~mL}, 0.650 \mathrm{mmol}$ ) was added while stirring. After $20 \mathrm{~min}$, volatiles were removed in vacuo. The material was washed with $2 \times 10 \mathrm{~mL} n$-pentane to afford bright purple powder $(0.132 \mathrm{~g}, 0.151 \mathrm{mmol}, 81 \%)$ assigned as ( $\left.{ }^{\mathrm{BBN}} \mathrm{PDP}{ }^{\mathrm{BBu}}\right) \mathrm{VCl}_{2}\left(\mathrm{NH}_{3}\right)_{2}(\mathrm{THF})$. Single, X-ray quality crystals were obtained by diffusing diethyl ether into a THF solution of $\left({ }^{B B N}{ }^{(B D P}{ }^{t B u}\right) V_{C l}\left(N_{3}\right)_{2}(T H F)$ at room temperature. The ${ }^{1} \mathrm{H}$ NMR spectrum $\left(25{ }^{\circ} \mathrm{C}\right.$, THF) of this complex is featureless. MALDI-TOF of $\mathrm{C}_{45} \mathrm{H}_{77} \mathrm{~N}_{7} \mathrm{~B}_{2} \mathrm{Cl}_{2} \mathrm{O}_{1} \mathrm{~V}_{1}-\left(2 \mathrm{NH}_{3}+\mathrm{OC}_{4} \mathrm{H}_{8}\right)$ : Calc. 768.409; Found 767.990. Selected IR data $(\mathrm{KBr}) \mathrm{v}=3357(\mathrm{~N}-\mathrm{H})$, $3145(\mathrm{~N}-\mathrm{H}), 2916,1607,1451,1151,873,792 \mathrm{~cm}^{-1}$. UV-Vis (THF, ambient temperature; $\lambda_{\max }$ molar absorptivity): $674 \mathrm{~nm}, 1700 \mathrm{M}^{-1} \mathrm{~cm}^{-1} ; 580 \mathrm{~nm}, 1200 \mathrm{M}^{-1} \mathrm{~cm}^{-1} ; 325 \mathrm{~nm}, 12900 \mathrm{M}^{-1} \mathrm{~cm}^{-1} ; 316 \mathrm{~nm}, 12600 \mathrm{M}^{-}$ ${ }^{1} \mathrm{~cm}^{-1} \cdot \mu_{\text {eff }}=3.75+/-0.05 \mu_{\mathrm{B}}\left(25^{\circ} \mathrm{C}, \mathrm{THF}\right)$.

Synthesis of ( $\left.{ }^{\text {BBN PDP }}{ }^{\text {BBu}}\right) \mathbf{V C l}_{2}\left(\mathrm{~N}_{2} \mathrm{H}_{4}\right)$ (THF). A $20 \mathrm{~mL}$ scintillation vial was charged with ( $\left.{ }^{\mathrm{BBN} P D P}{ }^{\mathrm{tBu}}\right) \mathrm{VCl}_{3}(0.228$ $\mathrm{g}, 0.283 \mathrm{mmol})$ and $6 \mathrm{~mL} \mathrm{THF}$ and frozen. Upon thawing, potassium graphite $(0.039 \mathrm{~g}, 0.289 \mathrm{mmol})$ was added resulting in a rapid color change to deep blue from yellow-green. The reaction was stirred for 10 min, filtered, and dried. The material was extracted into $4 \mathrm{~mL}$ toluene, filtered, and dried. The material was redissolved in $2 \mathrm{~mL}$ of THF and hydrazine ( $0.159 \mathrm{M}$ stock solution in THF, $1.650 \mathrm{~mL}, 0.262 \mathrm{mmol}$ ) was added while stirring. After $10 \mathrm{~min}$, volatiles were removed in vacuo. The material was washed with $2 \times 10$ $\mathrm{mL} n$-pentane to afford bright blue/purple powder $(0.206 \mathrm{~g}, 0.236 \mathrm{mmol}, 83 \%)$ assigned as $\left({ }^{\text {BBNPDP }}{ }^{\mathrm{tBu}}\right) \mathrm{VCl}_{2}\left(\mathrm{~N}_{2} \mathrm{H}_{4}\right)(\mathrm{THF})$. Single, $\mathrm{X}$-ray quality crystals were obtained by diffusing $n$-pentane into a THF solution of ( $\left.{ }^{\mathrm{BBN} P D P} \mathrm{PBU}^{\mathrm{Bu}}\right) \mathrm{VCl}_{2}\left(\mathrm{~N}_{2} \mathrm{H}_{4}\right)$ (THF) at room temperature. The ${ }^{1} \mathrm{H}$ NMR spectrum $\left(25{ }^{\circ} \mathrm{C}\right.$, THF) of this complex is featureless. MALDI-TOF of $\mathrm{C}_{45} \mathrm{H}_{75} \mathrm{~N}_{7} \mathrm{~B}_{2} \mathrm{Cl}_{2} \mathrm{O}_{1} \mathrm{~V}_{1}-\left(\mathrm{N}_{2} \mathrm{H}_{4}+\mathrm{OC}_{4} \mathrm{H}_{8}\right)$ : Calc. 768.409; Found 767.974. Selected IR data $(\mathrm{KBr}) \mathrm{v}=3301(\mathrm{~N}-\mathrm{H}), 3281(\mathrm{~N}-\mathrm{H}), 3243(\mathrm{~N}-\mathrm{H}), 3154(\mathrm{~N}-\mathrm{H}), 3116(\mathrm{~N}-\mathrm{H}), 3066(\mathrm{~N}-\mathrm{H}), 1606$, $1575,1492,1450,1248,897,791 \mathrm{~cm}^{-1}$. The poor solubility of this compound precluded accurate solution magnetic susceptibility measurements. UV-Vis (THF, ambient temperature; $\lambda_{\max }$, molar absorptivity): 672 $\mathrm{nm}, 1800 \mathrm{M}^{-1} \mathrm{~cm}^{-1} ; 570 \mathrm{~nm}, 1300 \mathrm{M}^{-1} \mathrm{~cm}^{-1} ; 326 \mathrm{~nm}, 14000 \mathrm{M}^{-1} \mathrm{~cm}^{-1} ; 315 \mathrm{~nm}, 13200 \mathrm{M}^{-1} \mathrm{~cm}^{-1}$. 


\section{( $\left.{ }^{\mathrm{BBN} P D P \mathrm{PBu}}\right) \mathrm{FeBr}_{2}\left(\mathrm{NH}_{2} \mathrm{Ph}\right)_{2}$}

a) Synthesis and Characterization: $A 20 \mathrm{~mL}$ scintillation vial was charged with ( $\left.{ }^{\mathrm{BBN} P D P}{ }^{\mathrm{tBu}}\right) \mathrm{FeBr}_{2}(0.063$ g, $0.073 \mathrm{mmol}$ ) and $2 \mathrm{~mL}$ THF. An initial ${ }^{1} \mathrm{H}$ NMR spectrum of this material was obtained. While stirring, aniline $(0.0133 \mathrm{~mL}, 0.146 \mathrm{mmol})$ was added and an ${ }^{1} \mathrm{H}$ NMR spectrum recorded after $10 \mathrm{~min}$. The ${ }^{1} \mathrm{H}$ NMR resonances reported are for the initial in situ ${ }^{1} \mathrm{H}$ NMR spectrum while the reported IR spectrum corresponds to the material after the initial precipitation (see below). Single, X-ray quality crystals were obtained by repeating the reaction in $\mathrm{C}_{6} \mathrm{D}_{6}$ in a sealed J-Young NMR tube and storing the tube overnight at ambient temperature. ${ }^{1} \mathrm{H}$ NMR resonances from this in situ reaction are also reported. ${ }^{1} \mathrm{H}$ NMR (THF, $\left.25^{\circ} \mathrm{C}\right) \delta=-31.82(30,1 \mathrm{H}, p$-pyr-CH), -8.51 (580), -3.84 (55), -3.20 (470), -0.66 (54), -0.25 (29), $0.16(31), 0.40(40), 9.65\left(14,18 \mathrm{H}, \mathrm{C}\left(\mathrm{CH}_{3}\right)_{3}\right), 31.76\left(400,4 \mathrm{H}, \mathrm{N}-\mathrm{CH}_{2}\right), 44.21(63,2 \mathrm{H}$, pyridine- or pyrazole-CH), $51.80(43,2 \mathrm{H}$, pyridine- or pyrazole- $\mathrm{CH}) .{ }^{1} \mathrm{H}$ NMR $\left(\mathrm{C}_{6} \mathrm{D}_{6}, 25^{\circ} \mathrm{C}\right) \delta=-33.43(56,1 \mathrm{H}, p$-pyr$\mathrm{CH}),-15.14(366),-7.41$ (480), -3.09 (77), -0.84 (64), $0.03(60), 0.58$ (71), 0.87 (26), 1.25 (47), 4.68 (131), $4.96(72), 8.86\left(38,18 \mathrm{H}, \mathrm{C}\left(\mathrm{CH}_{3}\right)_{3}\right), 30.44\left(400,4 \mathrm{H}, \mathrm{N}-\mathrm{CH}_{2}\right), 43.07$ (73, 2H, pyridine- or pyrazole$\mathrm{CH}), 50.97(76,2 \mathrm{H}$, pyridine- or pyrazole- $\mathrm{CH})$. Selected IR data $(\mathrm{KBr}) \mathrm{v}=3310(\mathrm{~N}-\mathrm{H}), 3196(\mathrm{~N}-\mathrm{H}), 3128$ (N-H), 1606, 1575, 1495, 1454, 1241, 1195, 794, $693 \mathrm{~cm}^{-1}$.

b) Reversibility - Vacuum and Washing: Using the solution described above, the product was then precipitated from THF with $18 \mathrm{~mL} n$-pentane and dried in vacuo for $10 \mathrm{~min}$. A small portion of the material was used to 1 ) acquire an ${ }^{1} \mathrm{H}$ NMR spectrum, and 2) acquire an infrared spectrum ( $\left.\mathrm{KBr}\right)$. The remaining material was dissolved in $2 \mathrm{~mL}$ THF, precipitated again with $18 \mathrm{~mL} n$-pentane, and dried for $10 \mathrm{~min}$ in vacuo. A small portion of the material was used to acquire an ${ }^{1} \mathrm{H}$ NMR spectrum. An overlay of the ${ }^{1} \mathrm{H}$ NMR spectra can be found below.

c) Titration Experiment: Stock solutions of $\left({ }^{B B N} \mathrm{PDP}^{\mathrm{tBu}}\right) \mathrm{FeBr}_{2}\left(3.01183 \times 10^{-3} \mathrm{M}, \mathrm{THF}\right)$ and aniline (2.1905 $\left.x 10^{-2} \mathrm{M}, \mathrm{THF}\right)$ were prepared. Portions of ( $\left.{ }^{\mathrm{BBN}} \mathrm{PDP}{ }^{\mathrm{tBu}}\right) \mathrm{FeBr}_{2}(1.000 \mathrm{~mL}$ each) were placed in six separate $20 \mathrm{~mL}$ scintillation vials. Increasing equivalents of aniline were then added to each: $0.00,0.50,1.00$, $1.25,1.50$, and 2.00 equivalents. The solutions were left for $30 \mathrm{~min}$, then ${ }^{1} \mathrm{H}$ NMR spectra were acquired. An overlay of the spectra is provided below.

Synthesis of ( $\left.{ }^{B B N P D P} P^{t B u}\right) \mathbf{M n}\left(\mathbf{N H}_{2}\right)_{2}$. A $20 \mathrm{~mL}$ scintillation vial was charged with ( $\left.{ }^{\mathrm{BBN} P D P}{ }^{\mathrm{BBu}}\right) \mathrm{MnBr}_{2}\left(\mathrm{NH}_{3}\right)_{2}$ $(0.202 \mathrm{~g}, 0.225 \mathrm{mmol})$ and $8 \mathrm{~mL}$ THF and frozen. A separate vial was charged with potassium bis(trimethylsilyl)amide $(0.090 \mathrm{~g}, 0.451 \mathrm{mmol})$ and $4 \mathrm{~mL}$ THF and frozen. Upon thawing, the solution of potassium bis(trimethylsilyl)amide was added to the solution of ( $\left.{ }^{\mathrm{BBN} P D P}{ }^{\mathrm{BBu}}\right) \mathrm{MnBr}_{2}\left(\mathrm{NH}_{3}\right)_{2}$, resulting in a rapid color change from colorless to light yellow, and stirred for $20 \mathrm{~min}$. Volatiles were removed in vacuo and the material extracted into $15 \mathrm{~mL} \mathrm{DCM}$, filtered, and dried. The solid was washed with $3 \times 10 \mathrm{~mL} n$ pentane to afford off-white powder $(0.138 \mathrm{~g}, 0.188 \mathrm{mmol}, 84 \%)$ assigned as ( $\left.{ }^{\mathrm{BBN} P D P}{ }^{\mathrm{BBu}}\right) \mathrm{Mn}\left(\mathrm{NH}_{2}\right)_{2}$. Single, $\mathrm{X}$-ray quality crystals were obtained from layering a concentrated DCM solution with $n$-pentane at room temperature. MALDI-TOF of $\mathrm{C}_{41} \mathrm{H}_{67} \mathrm{~N}_{7} \mathrm{~B}_{2} \mathrm{Mn}_{1}$ : Calc. 734.502; Found 734.292. ${ }^{1} \mathrm{H} \mathrm{NMR}\left(\mathrm{CDCl}_{3}, 25^{\circ} \mathrm{C}\right) \delta=3.38$ (595, broad, only observed resonance). Selected IR data (KBr) $v=3358(\mathrm{~N}-\mathrm{H}), 3318(\mathrm{~N}-\mathrm{H}), 3243(\mathrm{~N}-\mathrm{H})$, $3141(\mathrm{~N}-\mathrm{H}), 1608,1574,1454,799,739 \mathrm{~cm}^{-1}$. UV-Vis (THF, ambient temperature; $\lambda_{\max }$, molar absorptivity): $328 \mathrm{~nm}, 11300 \mathrm{M}^{-1} \mathrm{~cm}^{-1} ; 262 \mathrm{~nm}, 21500 \mathrm{M}^{-1} \mathrm{~cm}^{-1}$. $\mu_{\text {eff }}=5.43+/-0.28 \mu_{\mathrm{B}}\left(25^{\circ} \mathrm{C}\right.$, THF $)$. 
Synthesis of ( $\left.{ }^{\mathrm{BBN} P D P}{ }^{\mathrm{BBu}}\right) \mathrm{Fe}\left(\mathrm{NH}_{2}\right)_{2}$. A $20 \mathrm{~mL}$ scintillation vial was charged with ( $\left.{ }^{\mathrm{BBN} P D P}{ }^{\mathrm{BBu}}\right) \mathrm{FeBr}_{2}\left(\mathrm{NH}_{3}\right)_{2}(1.000$ $\mathrm{g}, 1.114 \mathrm{mmol}$ ) and $15 \mathrm{~mL}$ THF and chilled to $-35{ }^{\circ} \mathrm{C}$. A separate vial was charged with potassium bis(trimethylsilyl)amide $(0.445 \mathrm{~g}, 2.231 \mathrm{mmol})$ and $5 \mathrm{~mL}$ THF and chilled to $-35{ }^{\circ} \mathrm{C}$. The solution of potassium bis(trimethylsilyl)amide was added to the solution of ( $\left.{ }^{\mathrm{BBN} P D P}{ }^{\mathrm{tBu}}\right) \mathrm{FeBr}_{2}\left(\mathrm{NH}_{3}\right)_{2}$, resulting in a slight darkening of the yellow solution, and stirred for $60 \mathrm{~min}$. Volatiles were removed in vacuo and the material extracted into $40 \mathrm{~mL}$ DCM, filtered through Celite, and dried. The solid was washed with $4 \times 10 \mathrm{~mL} n$ pentane to afford salmon colored powder $(0.673 \mathrm{~g}, 0.915 \mathrm{mmol}, 82 \%)$ identified as ( $\left.{ }^{\mathrm{BBN} P D P} \mathrm{P}^{\mathrm{BBu}}\right) \mathrm{Fe}\left(\mathrm{NH}_{2}\right)_{2}$.

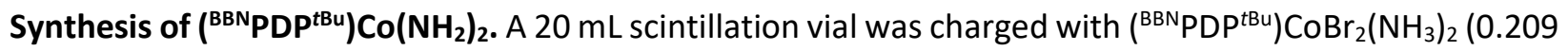
$\mathrm{g}, \quad 0.232 \mathrm{mmol}$ ) and $12 \mathrm{~mL}$ THF and frozen. A separate vial was charged with potassium bis(trimethylsilyl)amide $(0.093 \mathrm{~g}, 0.466 \mathrm{mmol})$ and $6 \mathrm{~mL} \mathrm{THF}$ and frozen. Upon thawing, the solution of potassium bis(trimethylsilyl)amide was added to the solution of ( $\left.{ }^{\mathrm{BBN} P D P}{ }^{\mathrm{tBu}}\right) \mathrm{CoBr}_{2}\left(\mathrm{NH}_{3}\right)_{2}$, resulting in a rapid color change from light green to light brown, and stirred for $20 \mathrm{~min}$. Volatiles were removed in vacuo and the material extracted into $16 \mathrm{~mL} \mathrm{DCM}$, filtered, and dried. The solid was washed with $3 \times 10 \mathrm{~mL} n$-pentane to afford light tan powder $(0.136 \mathrm{~g}, 0.184 \mathrm{mmol}, 79 \%)$ assigned as ( $\left.{ }^{\mathrm{BBN} P D P} \mathrm{P}^{\mathrm{BB}}\right) \mathrm{Co}\left(\mathrm{NH}_{2}\right)_{2}$. Single, $\mathrm{X}$-ray quality crystals were obtained from layering a concentrated DCM solution with $n$-pentane at room temperature. MALDI-TOF of $\mathrm{C}_{41} \mathrm{H}_{67} \mathrm{~N}_{7} \mathrm{~B}_{2} \mathrm{Co}_{1}$ : Calc. 738.498; Found 738.317. ${ }^{1} \mathrm{H}$ NMR $\left(\mathrm{CDCl}_{3}, 25{ }^{\circ} \mathrm{C}\right) \delta=-45.98(141,2 \mathrm{H})$, $22.92(169,2 \mathrm{H}),-20.54(22,1 \mathrm{H}, p$-pyridine- $\mathrm{CH}),-16.92(75,2 \mathrm{H}),-16.81(47,2 \mathrm{H}),-10.56\left(7,18 \mathrm{H}, \mathrm{C}\left(\mathrm{CH}_{3}\right)_{3}\right)$, $0.30(35,2 \mathrm{H}), 4.78(28,2 \mathrm{H}), 5.78(19,2 \mathrm{H}), 9.39(144,2 \mathrm{H}), 11.34(37,2 \mathrm{H}), 11.60(25,2 \mathrm{H}), 14.24(38,2 \mathrm{H})$, $15.98(26,2 \mathrm{H}), 16.95(33,2 \mathrm{H}), 24.99(37,2 \mathrm{H}), 34.47(29,2 \mathrm{H}), 36.38(281,2 \mathrm{H}), 43.89(138,2 \mathrm{H}), 44.78(28$, $2 \mathrm{H}), 60.92(2 \mathrm{O}, 2 \mathrm{H})$. Five $2 \mathrm{H}$ resonances are not observed. Selected IR data $(\mathrm{KBr}) \mathrm{v}=3356(\mathrm{~N}-\mathrm{H}), 3319(\mathrm{~N}-$ H), $3239(\mathrm{~N}-\mathrm{H}), 3144(\mathrm{~N}-\mathrm{H}), 1610,1573,1530,1454,800,739 \mathrm{~cm}^{-1}$. UV-Vis (THF, ambient temperature; $\lambda_{\max }$, molar absorptivity): $332 \mathrm{~nm}, 8700 \mathrm{M}^{-1} \mathrm{~cm}^{-1}$. $\mu_{\text {eff }}=4.44+/-0.05 \mu_{\mathrm{B}}\left(25^{\circ} \mathrm{C}\right.$, THF).

Synthesis of ( $\left.{ }^{\mathrm{BBN} P D P}{ }^{\mathrm{BBu}}\right) \mathrm{Zn}\left(\mathrm{NH}_{2}\right)_{2}$. A $20 \mathrm{~mL}$ scintillation vial was charged with ( $\left.{ }^{\mathrm{BBN} P D P}{ }^{\mathrm{BBu}}\right) \mathrm{ZnCl}_{2}\left(\mathrm{NH}_{3}\right)_{2}(0.252$ g, $0.308 \mathrm{mmol}$ ) and $10 \mathrm{~mL}$ THF and chilled to $-35{ }^{\circ} \mathrm{C}$. A separate vial was charged with potassium bis(trimethylsilyl)amide $(0.124 \mathrm{~g}, 0.622 \mathrm{mmol})$ and $5 \mathrm{~mL}$ THF and chilled to $-35{ }^{\circ} \mathrm{C}$. The solution of potassium bis(trimethylsilyl)amide was added to the solution of ( $\left.{ }^{\mathrm{BBN} P D P}{ }^{\mathrm{BBu}}\right) \mathrm{ZnCl}_{2}\left(\mathrm{NH}_{3}\right)_{2}$ and stirred for 25 min. Volatiles were removed in vacuo and the material extracted into $15 \mathrm{~mL} \mathrm{DCM}$, filtered, and dried. The solid was washed with $2 \times 10 \mathrm{~mL} n$-pentane to afford white powder $(0.162 \mathrm{~g}, 0.217 \mathrm{mmol}, 71 \%)$ assigned as $\left({ }^{\mathrm{BBN} P D P}{ }^{\mathrm{B} u}\right) \mathrm{Zn}\left(\mathrm{NH}_{2}\right)_{2}$. Single, $\mathrm{X}$-ray quality crystals were obtained from layering a concentrated DCM solution with hexamethyldisiloxane at room temperature. MALDI-TOF of $\mathrm{C}_{41} \mathrm{H}_{67} \mathrm{~N}_{7} \mathrm{~B}_{2} \mathrm{Zn}_{1}$ : Calc. 743.494; Found 743.313. ${ }^{1} \mathrm{H} \mathrm{NMR}\left(\mathrm{CDCl}_{3}, 25{ }^{\circ} \mathrm{C}\right) \delta=-0.86(\mathrm{t}, J=11.2,2 \mathrm{H}, \mathrm{B}-\mathrm{CH}),-0.24(\mathrm{~s}, 2 \mathrm{H}, \mathrm{NH}),-0.50(\mathrm{~d}, J=7.6$, $2 \mathrm{H}, \mathrm{B}-\mathrm{CH}), 0.75(\mathrm{t}, \mathrm{J}=10.4,2 \mathrm{H}, \mathrm{B}-\mathrm{CH}), 0.80(\mathrm{~s}, 2 \mathrm{H}, \mathrm{NH}), 1.32-1.47(\mathrm{~m}, 8 \mathrm{H}), 1.49\left(\mathrm{~s}, 18 \mathrm{H}, \mathrm{C}\left(\mathrm{CH}_{3}\right)_{3}\right), 1.62-1.78$ $(\mathrm{m}, 10 \mathrm{H}), 1.78-2.00(\mathrm{~m}, 10 \mathrm{H}), 2.31(\mathrm{~m}, 2 \mathrm{H}, \mathrm{CH}), 4.24(\mathrm{~m}, 2 \mathrm{H}, \mathrm{N}-\mathrm{CH}), 4.43(\mathrm{~m}, 2 \mathrm{H}, \mathrm{N}-\mathrm{CH}), 6.58(\mathrm{~s}, 2 \mathrm{H}$, pyrazole- $\mathrm{CH}$ ), $7.66(\mathrm{~d}, J=7.6,2 \mathrm{H}, m$-pyridine- $\mathrm{CH}), 8.00(\mathrm{t}, J=7.6,1 \mathrm{H}, p$-pyridine- $\mathrm{CH}) .{ }^{13} \mathrm{C} \mathrm{NMR}\left(\mathrm{CDCl}_{3}, 25\right.$ $\left.\left.{ }^{\circ} \mathrm{C}\right) \delta=17.83(\mathrm{~B}-\mathrm{CH}), 23.90(\mathrm{~B}-\mathrm{CH}), 24.97,25.88,25.91,29.98\left(\mathrm{C}_{(\mathrm{CH}}\right)_{3}\right), 30.75(\mathrm{~B}-\mathrm{CH}), 31.80,31.87,32.21$, 33.41, 33.44, $54.48\left(\mathrm{~N}-\mathrm{CH}_{2}\right), 101.44$ (pyrazole- $\left.\mathrm{CH}\right), 119.20$ ( $m$-pyridine- $\left.\mathrm{CH}\right), 140.76$ ( $p$-pyridine- $\left.\mathrm{CH}\right), 144.03$ $(\mathrm{Ar}-\mathrm{C}), 148.85(\mathrm{Ar}-\mathrm{C}), 156.53(\mathrm{Ar}-\mathrm{C}) .{ }^{11} \mathrm{~B}$ NMR $\left(\mathrm{CDCl}_{3}, 25^{\circ} \mathrm{C}\right) \delta=-7.60\left(\mathrm{~B}-\mathrm{NH}_{2}\right)$. Selected IR data $(\mathrm{KBr}) \mathrm{v}=$ $3326(\mathrm{~N}-\mathrm{H}), 3253(\mathrm{~N}-\mathrm{H}), 3143(\mathrm{~N}-\mathrm{H}), 3076,1609,1574,1536,1447,799,738 \mathrm{~cm}^{-1}$. UV-Vis (THF, ambient temperature; $\lambda_{\max }$, molar absorptivity): $330 \mathrm{~nm}, 9000 \mathrm{M}^{-1} \mathrm{~cm}^{-1}$. 
Synthesis of $\left[\mathrm{Cs}(18-\mathrm{crown}-6)_{2}\right]\left[\left({ }^{\mathrm{BBN}} \mathrm{PDP} \mathrm{P}^{\mathrm{BBu}}\right) \mathrm{Fe}\left(\mathrm{NH}_{2}\right)_{2}\right]$. A $20 \mathrm{~mL}$ scintillation vial was charged with $\left({ }^{B B N} P D P^{t B u}\right) F e\left(N_{2}\right)_{2}(0.078 \mathrm{~g}, 0.106 \mathrm{mmol})$ and $8 \mathrm{~mL}$ THF and frozen. Upon thawing, $\mathrm{CsC}_{8}(0.024,0.105$ $\mathrm{mmol})$ was added resulting in a rapid color change from light orange to dark brown. After $3 \mathrm{~min}, 18$-crown$6(0.056 \mathrm{~g}, 0.212 \mathrm{mmol})$ was added and the solution stirred for $30 \mathrm{~min}$, filtered, and dried. The solid was washed with $2 \times 10 \mathrm{~mL}$ diethyl ether and dried to afford dark brown powder $(0.109 \mathrm{~g}, 0.078 \mathrm{mmol}, 74 \%)$ assigned as $\left[\mathrm{Cs}(18-\mathrm{crown}-6)_{2}\right]\left[\left({ }^{\mathrm{BBN}} \mathrm{PDP}{ }^{\mathrm{BB}}\right) \mathrm{Fe}\left(\mathrm{NH}_{2}\right)_{2}\right]$. Single, $\mathrm{X}$-ray quality crystals were obtained from layering a THF solution with diethyl ether at $-35{ }^{\circ} \mathrm{C}$. Cation metathesis with $\left[\mathrm{Bu}_{4} \mathrm{~N}\right][\mathrm{I}]$ was performed by adding equimolar $\left[\mathrm{Bu}_{4} \mathrm{~N}\right][\mathrm{I}]$ to the reduction mixture (without crown ether) and stirring for 15 min followed by filtration to afford the more soluble $\left[\mathrm{Bu}_{4} \mathrm{~N}\right]\left[\left({ }^{\left({ }^{B N} \mathrm{PDP}\right.}{ }^{\mathrm{BBu}}\right) \mathrm{Fe}\left(\mathrm{NH}_{2}\right)_{2}\right]$. Alternately, $\mathrm{KC}_{8}$ can be used in place of $\mathrm{CsC}_{8}$ to form the potassium salt. ${ }^{1} \mathrm{H}$ NMR for $\left[\mathrm{Bu}_{4} \mathrm{~N}\right]\left[\left({ }^{(B B N P D P}{ }^{\mathrm{BBu}}\right) \mathrm{Fe}\left(\mathrm{NH}_{2}\right)_{2}\right]\left(\mathrm{THF}, 25^{\circ} \mathrm{C}\right) \delta=-95.05$ $(231,2 \mathrm{H}),-34.17(143,2 \mathrm{H}),-24.62(890,1 \mathrm{H}),-13.84(54,2 \mathrm{H}),-9.24(41,2 \mathrm{H}),-5.48(34,2 \mathrm{H}),-3.06(27,2 \mathrm{H})$, $-2.65(121,2 \mathrm{H}),-2.17(40,2 \mathrm{H}),-2.12(22,4 \mathrm{H}),-1.98(24,2 \mathrm{H}),-1.53(30,2 \mathrm{H}),-1.08(36,2 \mathrm{H}),-0.67(19,2 \mathrm{H})$, $-0.04(25,2 \mathrm{H}), 0.07(64,2 \mathrm{H}), 0.98(20,12 \mathrm{H}, \mathrm{TBA}), 6.50(26,2 \mathrm{H}), 10.06\left(11,18 \mathrm{H}, \mathrm{C}\left(\mathrm{CH}_{3}\right)_{3}\right), 11.37(61,2 \mathrm{H})$, $11.61(210,2 \mathrm{H}), 17.97(62,2 \mathrm{H}), 21.51(126,2 \mathrm{H}), 75.91(44,2 \mathrm{H})$. Selected IR data for [K(18-crown-

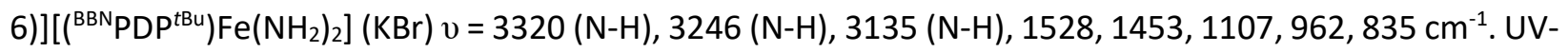
Vis for [Cs(18-crown-6) $\left.\left.)_{2}\right]\left({ }^{\text {BBN } P D P}{ }^{\text {tBu }}\right) \mathrm{Fe}\left(\mathrm{NH}_{2}\right)_{2}\right]$ (THF, ambient temperature; $\lambda_{\max }$, molar absorptivity): 335 $\mathrm{nm}, 7425 \mathrm{M}^{-1} \mathrm{~cm}^{-1} ; 418 \mathrm{~nm}, 3200 \mathrm{M}^{-1} \mathrm{~cm}^{-1} ; 490 \mathrm{~nm}, 3818 \mathrm{M}^{-1} \mathrm{~cm}^{-1} ; 510 \mathrm{~nm}, 3814 \mathrm{M}^{-1} \mathrm{~cm}^{-1} ; 584 \mathrm{~nm}, 1983 \mathrm{M}^{-}$ ${ }^{1} \mathrm{~cm}^{-1}$. $\mu_{\text {eff }}$ of $\left[\mathrm{Bu}_{4} \mathrm{~N}\right]\left[\left(^{\mathrm{BBN} P D P^{t B u}}\right) \mathrm{Fe}\left(\mathrm{NH}_{2}\right)_{2}\right]=4.29+/-0.17 \mu_{\mathrm{B}}\left(25^{\circ} \mathrm{C}\right.$, THF $)$.

Synthesis of $[K(2,2,2-c r y p t)]\left[\left(\right.\right.$ benzyl-BBNPDP $\left.\left.{ }^{\mathrm{BBu}}\right) \mathrm{Fe}\left(\mathrm{NH}_{2}\right)_{2}\right]$. A $20 \mathrm{~mL}$ scintillation vial was charged with $\left({ }^{\left.\mathrm{BBN} P D P^{\mathrm{Bu}}\right)}\right) \mathrm{Fe}\left(\mathrm{NH}_{2}\right)_{2}(0.168 \mathrm{~g}, 0.228 \mathrm{mmol})$ and $10 \mathrm{~mL}$ THF and stirred until fully dissolved. 2,2,2-Cryptand $(0.085 \mathrm{~g}, 0.226 \mathrm{mmol})$ was then added and the solution frozen. A separate vial was charged with benzylpotassium $(0.030 \mathrm{~g}, 0.230 \mathrm{mmol})$ and $3 \mathrm{~mL}$ THF and frozen. Upon thawing, the solution of benzylpotassium was added to the iron-containing solution and stirred for $20 \mathrm{~min}$. Volatiles were removed in vacuo and the resulting solid washed with $2 \times 10 \mathrm{~mL} n$-pentane to afford tan/orange powder $(0.239 \mathrm{~g}$, $0.192 \mathrm{mmol}, 84 \%)$ assigned as [K(2,2,2-crypt)][(benzyl-BBNPDP $\left.\left.{ }^{\mathrm{BBu}}\right) \mathrm{Fe}\left(\mathrm{NH}_{2}\right)_{2}\right]$. Single, $\mathrm{X}$-ray quality crystals were obtained from slow diffusion of $n$-pentane into a THF solution at room temperature. Other crown ethers can be used in place of 2,2,2-cryptand. ${ }^{1} \mathrm{H}$ NMR for [K(18-crown-6)][(benzyl-- $\left.\left.{ }^{\mathrm{BBN} P D P}{ }^{\mathrm{BB}}\right) \mathrm{Fe}\left(\mathrm{NH}_{2}\right)_{2}\right]$ $\left(\mathrm{THF}, 25{ }^{\circ} \mathrm{C}\right.$ ) $\delta=$ (all single proton $(1 \mathrm{H})$ resonances unless otherwise noted) -40.95 (222), -40.37 (226), 39.70 (57), 38.27 (55), -18.23 (30, Ph-CH), -18.09 (31, Ph-CH), -10.88 (66), -10.59 (65), -6.87 (43), -6.48 (44), -3.38 (40), -2.89 (33), -2.75 (38), -2.60 (44), -2.11 (172), -1.93 (37), -1.65 (33), -1.57 (18), -1.44 (29), $0.61(23),-0.33(22), 2.86(31,24 \mathrm{H}, 18$-crown-6), 3.98 (d, $J=6,2 \mathrm{H}, o$-phenyl-CH), $5.43(\mathrm{t}, J=7,1 \mathrm{H}, p$ phenyl-CH), $5.62(\mathrm{t}, J=6,2 \mathrm{H}, m$-phenyl- $\mathrm{CH}), 9.65(29), 10.02(28), 10.71\left(12,9 \mathrm{H}, \mathrm{C}\left(\mathrm{CH}_{3}\right)_{3}\right), 10.73(12,9 \mathrm{H}$, $\left.\mathrm{C}\left(\mathrm{CH}_{3}\right)_{3}\right), 24.72$ (240), 25.36 (214), 32.51 (236), 32.79 (204), 38.17 (123), 38.45 (99), 46.15 (47), 46.86 (51). Selected IR data for [K(18-crown-6)][(benzyl-- $\left.\left.{ }^{\mathrm{BB} P D P}{ }^{\mathrm{tBu}}\right) \mathrm{Fe}\left(\mathrm{NH}_{2}\right)_{2}\right](\mathrm{KBr}) \mathrm{v}=3323(\mathrm{~N}-\mathrm{H}), 3243(\mathrm{~N}-\mathrm{H}), 3131$ (N-H), 1639, 1611, 1585, 1530, 1360, 949, 752, $701 \mathrm{~cm}^{-1}$. UV-Vis (THF, ambient temperature; $\lambda_{\max }$, molar absorptivity): $345 \mathrm{~nm}, 7700 \mathrm{M}^{-1} \mathrm{~cm}^{-1}$. $\mu_{\text {eff }}$ for $[K(2,2,2$-crypt $)]\left[\left(\right.\right.$ benzyl- $\left.\left.{ }^{B B N} P D P^{\mathrm{BBu}}\right) \mathrm{Fe}\left(\mathrm{NH}_{2}\right)_{2}\right]=5.12+/-0.08 \mu_{\mathrm{B}}$ $\left(25^{\circ} \mathrm{C}, \mathrm{THF}\right)$. 
Synthesis of $\left[\mathrm{Cs}(18-\mathrm{crown}-6)_{2}\right]\left[\left({ }^{\mathrm{BBN}} \mathrm{PDP}{ }^{\mathrm{tBu}}\right) \mathrm{Co}\left(\mathrm{NH}_{2}\right)_{2}\right]$. A $20 \mathrm{~mL}$ scintillation vial was charged with $\left({ }^{B B N}\right.$ PDP $\left.{ }^{t B u}\right) \mathrm{Co}\left(\mathrm{NH}_{2}\right)_{2}(0.036 \mathrm{~g}, 0.049 \mathrm{mmol})$ and $10 \mathrm{~mL}$ THF. While stirring, 18-crown-6 (0.026 g, 0.098 $\mathrm{mmol})$ was added and the solution frozen. Upon thawing, $\mathrm{CsC}_{8}(0.011 \mathrm{~g}, 0.048 \mathrm{mmol})$ was added resulting in a rapid color change from light brown to dark brown. After stirring at room temp for $15 \mathrm{~min}$, the solution was filtered. To induce precipitation, $12 \mathrm{~mL} n$-pentane was added. The solid was collected and dried to afford brown powder $(0.045 \mathrm{~g}, 0.032 \mathrm{mmol}, 66 \%)$ assigned as [Cs(18-crown-6) $\left.{ }_{2}\right]\left[\left({ }^{\mathrm{BBN}} \mathrm{PDP} \mathrm{P}^{\mathrm{BBu}}\right) \mathrm{Co}\left(\mathrm{NH}_{2}\right)_{2}\right]$. Single, $\mathrm{X}$-ray quality crystals were obtained by layering a THF solution with diethyl ether at $-35{ }^{\circ} \mathrm{C}$. Cation metathesis with $\left[\mathrm{Bu}_{4} \mathrm{~N}\right][\mathrm{I}]$ was performed by adding equimolar $\left[\mathrm{Bu}_{4} \mathrm{~N}\right][\mathrm{I}]$ to the reduction mixture (without crown ether) and stirring for $15 \mathrm{~min}$ followed by filtration to afford the more soluble $\left[\mathrm{Bu}_{4} \mathrm{~N}\right]\left[\left({ }^{\mathrm{BBN} P D P} \mathrm{PBu}^{\mathrm{B} u}\right) \mathrm{Co}\left(\mathrm{NH}_{2}\right)_{2}\right] .{ }^{1} \mathrm{H}$ NMR for $\left[\mathrm{Bu}_{4} \mathrm{~N}\right]\left[\left({ }^{\mathrm{BBN} P D P} \mathrm{P}^{\mathrm{tBu}}\right) \mathrm{Co}\left(\mathrm{NH}_{2}\right)_{2}\right]\left(\mathrm{THF}, 25{ }^{\circ} \mathrm{C}\right) \delta=-88.89(157,2 \mathrm{H})$, $40.0(800,2 \mathrm{H}),-16.33(254,2 \mathrm{H}),-11.90(55,2 \mathrm{H}),-1.75\left(11,18 \mathrm{H}, \mathrm{C}\left(\mathrm{CH}_{3}\right)_{3}\right), 0.82\left(15,12 \mathrm{H}, \mathrm{Bu}_{4} \mathrm{~N}\right), 1.16(65$, $1 \mathrm{H}, p$-pyridine- $\mathrm{CH}), 1.39\left(25,8 \mathrm{H}, \mathrm{Bu}_{4} \mathrm{~N}\right), 2.23\left(26,8 \mathrm{H}, \mathrm{Bu}_{4} \mathrm{~N}\right), 2.48(20,2 \mathrm{H}), 2.83(27,2 \mathrm{H}), 3.00(17,2 \mathrm{H})$, $3.04(29,2 \mathrm{H}), 4.18\left(23,8 \mathrm{H} \mathrm{Bu}_{4} \mathrm{~N}\right), 4.41(29,2 \mathrm{H}), 5.73(76,2 \mathrm{H}), 5.82(42,2 \mathrm{H}), 6.82(24,2 \mathrm{H}), 6.94(56,2 \mathrm{H})$, $8.23(132,2 \mathrm{H}), 8.65(23,2 \mathrm{H}), 12.12(28,2 \mathrm{H}), 14.74(42,2 \mathrm{H}), 15.46(104,2 \mathrm{H}), 49.07(59,2 \mathrm{H})$. Selected IR data for $\left[\mathrm{Bu}_{4} \mathrm{~N}\right]\left[\left(^{\mathrm{BBN} P D P^{t B u}}\right) \mathrm{Co}\left(\mathrm{NH}_{2}\right)_{2}\right](\mathrm{KBr}) \mathrm{v}=3325(\mathrm{~N}-\mathrm{H}), 3246(\mathrm{~N}-\mathrm{H}), 1571,1531,1456,1301,1233,1190$, 1094, 1041, 942, 890, 744, $691 \mathrm{~cm}^{-1}$. UV-Vis for [Bu $\left.4 \mathrm{~N}\right]\left[\left({ }^{\mathrm{BBN}} \mathrm{PDP}{ }^{\mathrm{tBu}}\right) \mathrm{Co}\left(\mathrm{NH}_{2}\right)_{2}\right](\mathrm{THF}$, ambient temperature; $\lambda_{\text {max }}$ molar absorptivity): $341 \mathrm{~nm}, 7000 \mathrm{M}^{-1} \mathrm{~cm}^{-1} ; 446 \mathrm{~nm}$ (shoulder), $4100 \mathrm{M}^{-1} \mathrm{~cm}^{-1} ; 482,4300 \mathrm{M}^{-1} \mathrm{~cm}^{-1} ; 706$, $2333 \mathrm{M}^{-1} \mathrm{~cm}^{-1}$. $\mu_{\text {eff }}$ for $\left[\mathrm{Bu}_{4} \mathrm{~N}\right]\left[\left({ }^{\mathrm{BBN} P D P^{\mathrm{BB} u}}\right) \mathrm{Co}\left(\mathrm{NH}_{2}\right)_{2}\right]=3.22+/-0.07 \mu_{\mathrm{B}}\left(25^{\circ} \mathrm{C}, \mathrm{THF}\right)$.

\section{Oxidation of ( $\left.{ }^{\mathrm{BBN} P D P}{ }^{\mathrm{BBu}}\right) \mathrm{Fe}\left(\mathrm{NH}_{2}\right)_{2}$}

A) with PhICl 2 . A $20 \mathrm{~mL}$ scintillation vial was charged with ( $\left.{ }^{\mathrm{BBN} P D P}{ }^{\mathrm{tBu}}\right) \mathrm{Fe}\left(\mathrm{NH}_{2}\right)_{2}(0.029,0.039 \mathrm{mmol})$ and $2 \mathrm{~mL}$ THF and frozen. Upon thawing, $\mathrm{PhICl}_{2}(0.011 \mathrm{~g}, 0.040 \mathrm{mmol})$ was added. The solution was stirred for $15 \mathrm{hr}$. Analysis of the crude reaction mixture by ${ }^{1} \mathrm{H}$ NMR spectroscopy revealed formation of $\left({ }^{\text {BBNPDPtBu}}\right) \mathrm{FeCl}_{2}\left(\mathrm{NH}_{3}\right)_{2}$. A representative spectrum is shown below.

B) with $\left[\mathrm{Cp}_{2} \mathrm{Fe}\right]\left[\mathrm{BPh}_{4}\right]$ in presence of $\mathrm{PPN}-\mathrm{Cl}$. A $20 \mathrm{~mL}$ scintillation vial was charged with $\left({ }^{B B N} P D P{ }^{t B u}\right) \mathrm{Fe}\left(\mathrm{NH}_{2}\right)_{2}(0.024,0.033 \mathrm{mmol})$ and $4 \mathrm{~mL}$ THF. While stirring, bis(triphenylphosphine)iminium chloride $(0.036 \mathrm{~g}, 0.066 \mathrm{mmol})$ was added followed by ferrocenium tetraphenylborate $(0.033 \mathrm{~g}, 0.065$ $\mathrm{mmol})$. The solution was stirred for $30 \mathrm{~min}$ then an aliquot was filtered into an ${ }^{1} \mathrm{H}$ NMR tube. Analysis of the crude reaction mixture revealed formation of $\left({ }^{\mathrm{BBN} P D P}{ }^{\mathrm{Bu} u}\right) \mathrm{FeCl}_{2}\left(\mathrm{NH}_{3}\right)_{2}$. A representative spectrum is shown below.

C) with $\mathrm{AgSbF}_{6}$ in presence of PPN-Cl. A $20 \mathrm{~mL}$ scintillation vial was charged with ( $\left.{ }^{\mathrm{BBN} P D P}{ }^{\mathrm{tBu}}\right) \mathrm{Fe}\left(\mathrm{NH}_{2}\right)_{2}$ $(0.024,0.033 \mathrm{mmol})$ and $4 \mathrm{~mL} \mathrm{DCM}$. While stirring, bis(triphenylphosphine)iminium chloride $(0.036 \mathrm{~g}$, $0.066 \mathrm{mmol}$ ) was added followed by silver hexafluoroantimonate $(0.022 \mathrm{~g}, 0.064 \mathrm{mmol})$. The solution was stirred for $30 \mathrm{~min}$ then an aliquot was filtered into an ${ }^{1} \mathrm{H}$ NMR tube. Analysis of the crude reaction mixture revealed formation of $\left({ }^{\mathrm{BBN} P D P}{ }^{\mathrm{BBu}}\right) \mathrm{FeCl}_{2}\left(\mathrm{NH}_{3}\right)_{2}$. A representative spectrum is shown below. 
One-pot synthesis of (benzyl-BBNPDP $\left.{ }^{\mathrm{tBu}}\right) \mathrm{Fe}\left(\mathrm{NH}_{2}\right)_{2}$. A $20 \mathrm{~mL}$ scintillation vial was charged with $\left({ }^{B B N} P D P^{t B u}\right) F e\left(N_{2}\right)_{2}(0.250 \mathrm{~g}, 0.340 \mathrm{mmol})$ and $10 \mathrm{~mL}$ THF and stirred. A separate vial was charged with benzylpotassium $(0.044 \mathrm{~g}, 0.338 \mathrm{mmol}$ ) and $2 \mathrm{~mL}$ THF. Both vials were frozen. Upon thawing, the solution of benzylpotassium was added to the iron containing vial and the reaction stirred at room temperature for $20 \mathrm{~min}$. The solution was refrozen, and upon thawing, iodine $(0.170 \mathrm{mmol}$, THF stock solution) was added and the solution was stirred an additional 20 min resulting in formation of a white precipitate. The solution was filtered, dried, and washed with $3 \times 15 \mathrm{~mL} n$-pentane to afford tan powder $(0.171 \mathrm{~g}, 0.207$ mmol, 61\%) assigned as (benzyl- $\left.{ }^{B B N} P D P{ }^{t B u}\right) F e\left(N_{2}\right)_{2}$. Single, $\mathrm{X}$-ray quality crystals were obtained by diffusing $n$-pentane into a toluene solution at room temperature. MALDI-TOF of $\mathrm{C}_{48} \mathrm{H}_{73} \mathrm{~N}_{7} \mathrm{~B}_{2} \mathrm{Fe}_{1}$ : Calc. 825.546; Found 826.242. For $\mathrm{C}_{48} \mathrm{H}_{73} \mathrm{~N}_{7} \mathrm{~B}_{2} \mathrm{Fe}_{1}-\mathrm{NH}_{2}$ : Calc. 809.528; Found 809.180. For $\mathrm{C}_{48} \mathrm{H}_{66} \mathrm{D}_{7} \mathrm{~N}_{7} \mathrm{~B}_{2} \mathrm{Fe}_{1}$ : Calc. 832.590; Found 833.218. For $\mathrm{C}_{48} \mathrm{H}_{66} \mathrm{D}_{7} \mathrm{~N}_{7} \mathrm{~B}_{2} \mathrm{Fe}_{1}-\mathrm{NH}_{2}$ : Calc. 816.572; Found 816.411. ${ }^{1} \mathrm{H}$ NMR (THF, 25 $\left.{ }^{\circ} \mathrm{C}\right) \delta=-22.45(442,2 \mathrm{H}),-1.23(61,2 \mathrm{H}),-0.50(54,2 \mathrm{H}),-0.12(28,2 \mathrm{H}), 1.20(38,2 \mathrm{H}), 4.24\left(12,18 \mathrm{H}, \mathrm{C}\left(\mathrm{CH}_{3}\right)_{3}\right)$, $4.40(52,2 \mathrm{H}), 4.71(17,2 \mathrm{H}, \mathrm{o}$-phenyl- $\mathrm{CH}), 5.18(36,2 \mathrm{H}), 6.14(30,2 \mathrm{H}), 6.32(\mathrm{t}, \mathrm{J}=7,1 \mathrm{H}, p$-phenyl-CH), 6.57 $(\mathrm{t}, \mathrm{J}=6,2 \mathrm{H}, \mathrm{m}$-phenyl-CH), $10.84(216,2 \mathrm{H}), 12.42(149,2 \mathrm{H}), 13.87(28,2 \mathrm{H}), 22.38(26,1 \mathrm{H}, \mathrm{Ph}-\mathrm{CH}), 22.76$ (27, $1 \mathrm{H}, \mathrm{Ph}-\mathrm{CH}), 25.30(91,2 \mathrm{H}), 33.88(45,2 \mathrm{H}), 39.70(202,2 \mathrm{H}), 48.23(57,2 \mathrm{H})$. Selected IR data $(\mathrm{KBr}) \mathrm{v}=$ $3319(\mathrm{~N}-\mathrm{H}), 3242(\mathrm{~N}-\mathrm{H}) \mathrm{cm}^{-1}$. UV-Vis (THF, ambient temperature; $\lambda_{\max }$, molar absorptivity): $330 \mathrm{~nm}, 8800$ $\mathrm{M}^{-1} \mathrm{~cm}^{-1} . \mu_{\mathrm{eff}}=5.11+/-0.06 \mu_{\mathrm{B}}\left(25^{\circ} \mathrm{C}\right.$, THF $)$. Performing the intermediate oxidation with $\mathrm{I}_{2}$ in a sealed $\mathrm{J}$ Young NMR tube does not produce $\mathrm{H}_{2}$, as assessed by ${ }^{1} \mathrm{H}$ NMR. Deuteration of the benzyl substituent was performed in analogous fashion with $\mathrm{KC}_{7} \mathrm{D}_{7}$.

Synthesis of $\left[\left({ }^{B B N} P D P{ }^{t B u}\right) F e\left(N_{2}\right)_{2}\right]_{2}[\text { Li(THF)(12-crown-4) }]_{2}$. A $20 \mathrm{~mL}$ scintillation vial was charged with $\left({ }^{B B N P D P}{ }^{t B u}\right) F e\left(N_{2}\right)_{2}(0.050 \mathrm{~g}, 0.068 \mathrm{mmol})$ and $6 \mathrm{~mL}$ THF. While stirring, 12-crown-4 $(0.044 \mathrm{~mL}, 0.272$ $\mathrm{mmol}$ ) was added and the solution frozen. Upon thawing, lithium diisopropylamide (2.0 $\mathrm{M}$ solution in $\mathrm{THF} /$ heptane/ethylbenzene, $0.136 \mathrm{mmol}$ ) was added and warmed to room temperature resulting in a gradual color change to deep blue. After $10 \mathrm{~min}$, volatiles were removed in vacuo. The crude material was twice dissolved in minimal THF and precipitated with excess $n$-pentane. The blue powder was dried and analyzed by ${ }^{1} \mathrm{H}$ NMR spectroscopy and electronic absorption spectroscopy (see below). The title complex is formed in an approximate ratio of 3:1 with another unidentified paramagnetic molecule (based on ${ }^{1} \mathrm{H}$ NMR integrations). The title complex is highly unstable and further attempts at purification were unsuccessful. Single, $\mathrm{X}$-ray quality crystals were able to be obtained by layering a THF solution of the product with $n$-pentane at $-35^{\circ} \mathrm{C}$. The crystals rapidly desolvate if suspended in common X-ray oils and rapidly decompose when exposed to air. 

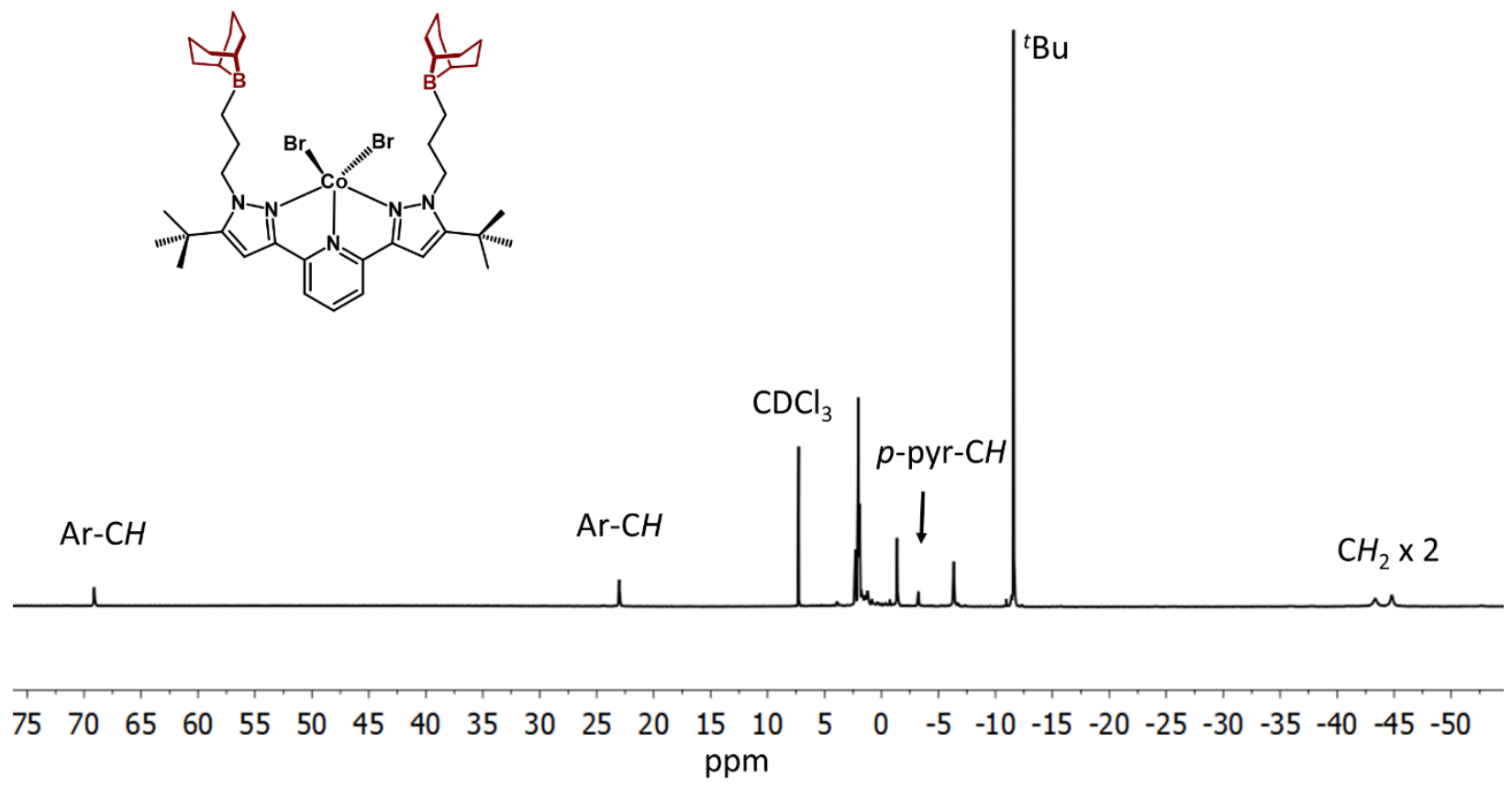

Figure $\mathrm{S} 1{ }^{1} \mathrm{H}$ NMR spectrum $\left(\mathrm{CDCl}_{3}, 25^{\circ} \mathrm{C}\right)$ of $\left({ }^{\mathrm{BBN} P D P} \mathrm{P}^{\mathrm{tBu}}\right) \mathrm{CoBr}_{2}$.

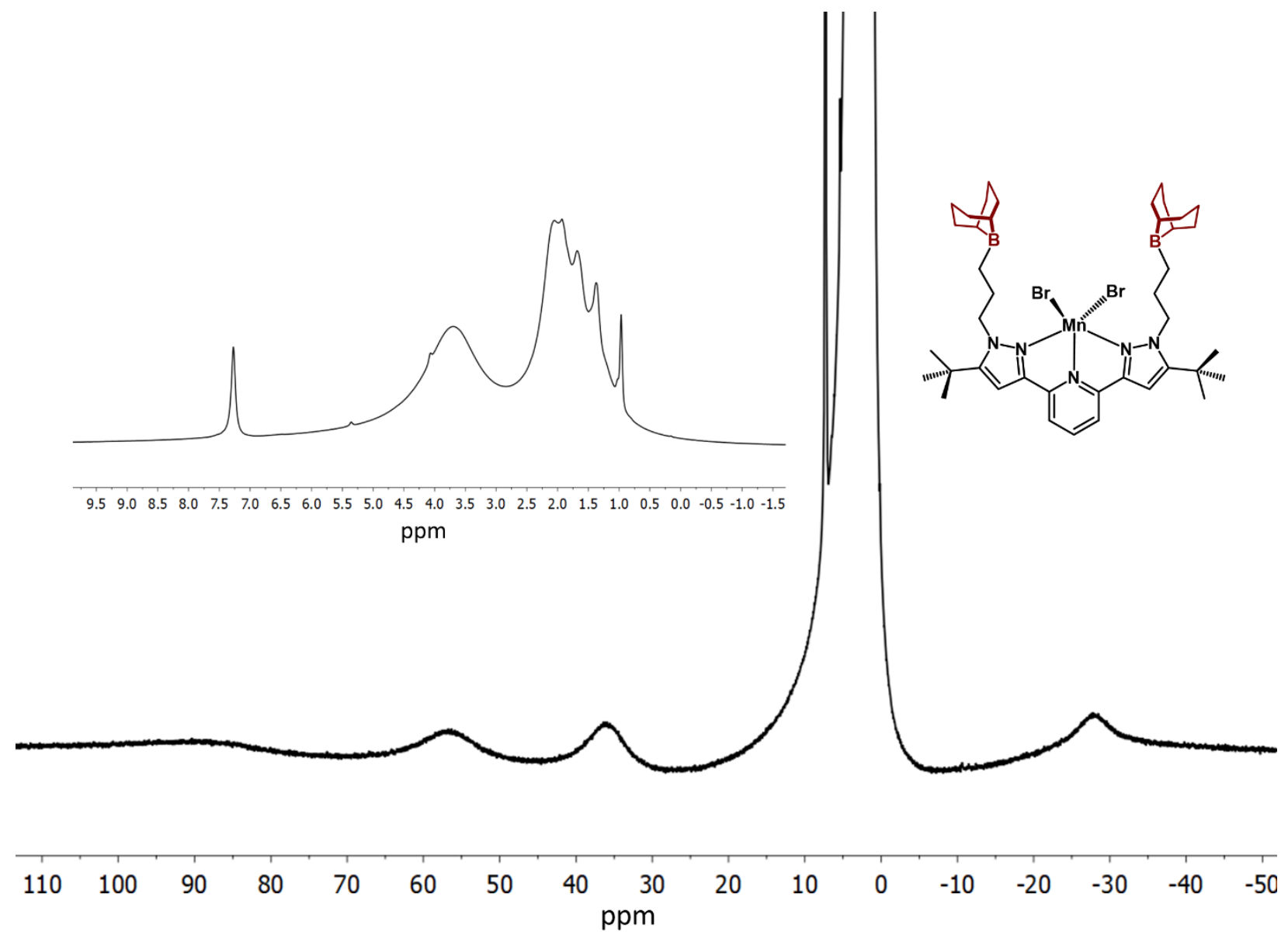

Figure S2 ${ }^{1} \mathrm{H} N M R$ spectrum $\left(\mathrm{CDCl}_{3}, 25^{\circ} \mathrm{C}\right)$ of $\left({ }^{\mathrm{BBN}} \mathrm{PDP}^{\mathrm{BBu}}\right) \mathrm{MnBr}_{2}$. 

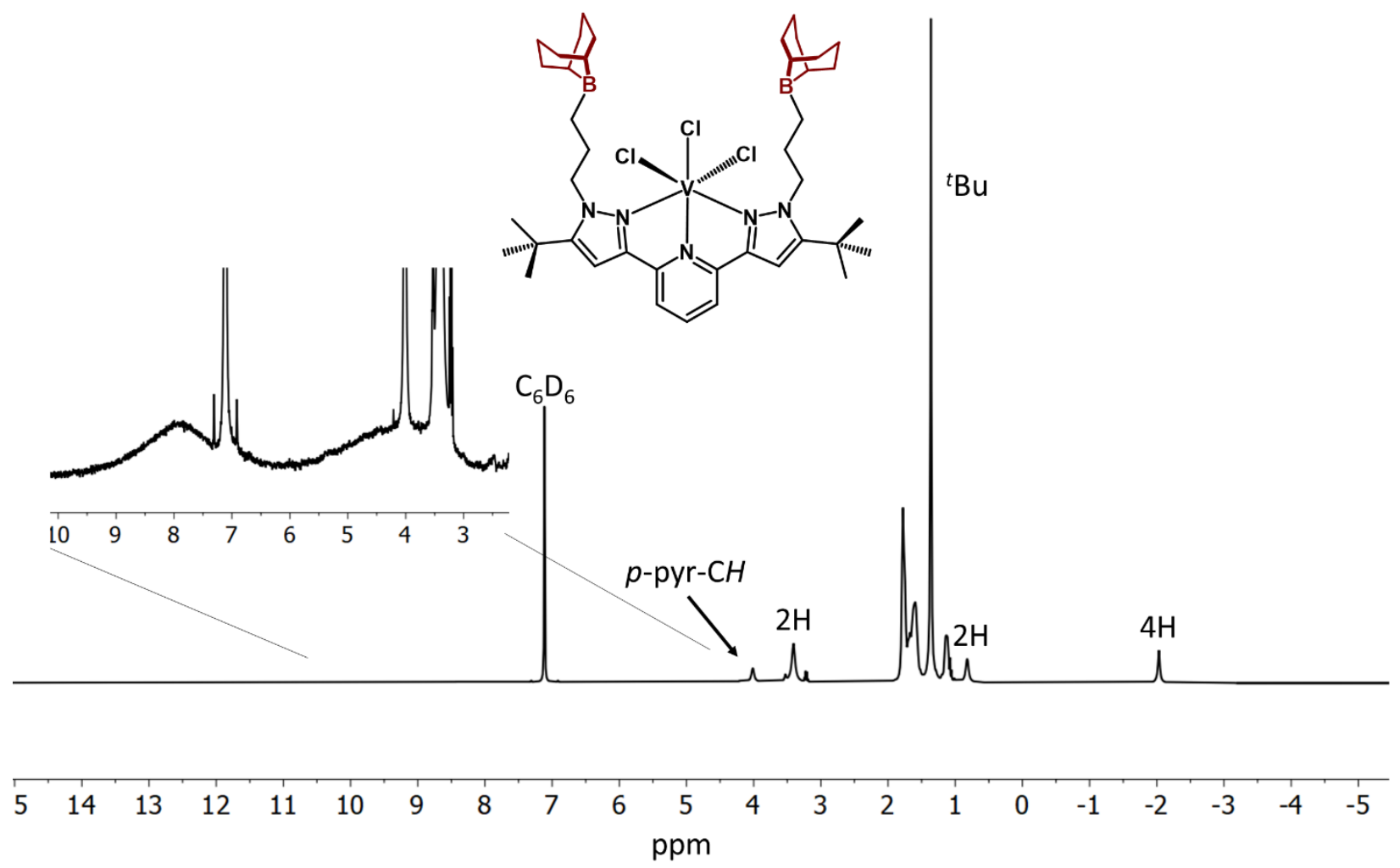

Figure $\mathrm{S} 3{ }^{1} \mathrm{H}$ NMR spectrum $\left(\mathrm{C}_{6} \mathrm{D}_{6}, 25^{\circ} \mathrm{C}\right)$ of $\left({ }^{\mathrm{BBN} P D P}{ }^{\mathrm{BBu}}\right) \mathrm{VCl}_{3}$.
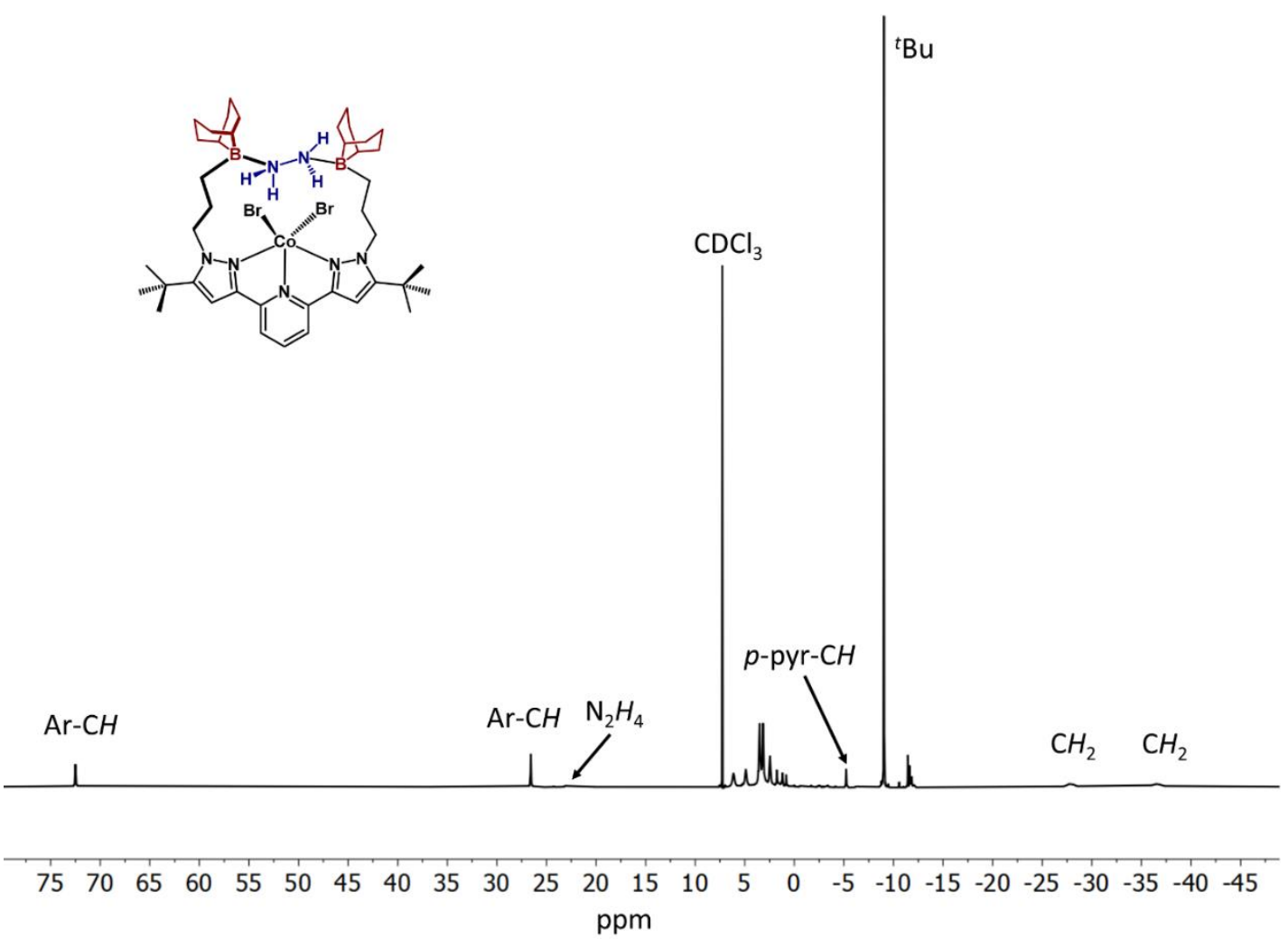

Figure $\mathrm{S} 4{ }^{1} \mathrm{H}$ NMR spectrum $\left(\mathrm{CDCl}_{3}, 25^{\circ} \mathrm{C}\right)$ of $\left({ }^{\mathrm{BBN} P D P}{ }^{\mathrm{tBu}}\right) \mathrm{CoBr}_{2}\left(\mathrm{~N}_{2} \mathrm{H}_{4}\right)$.

S16 


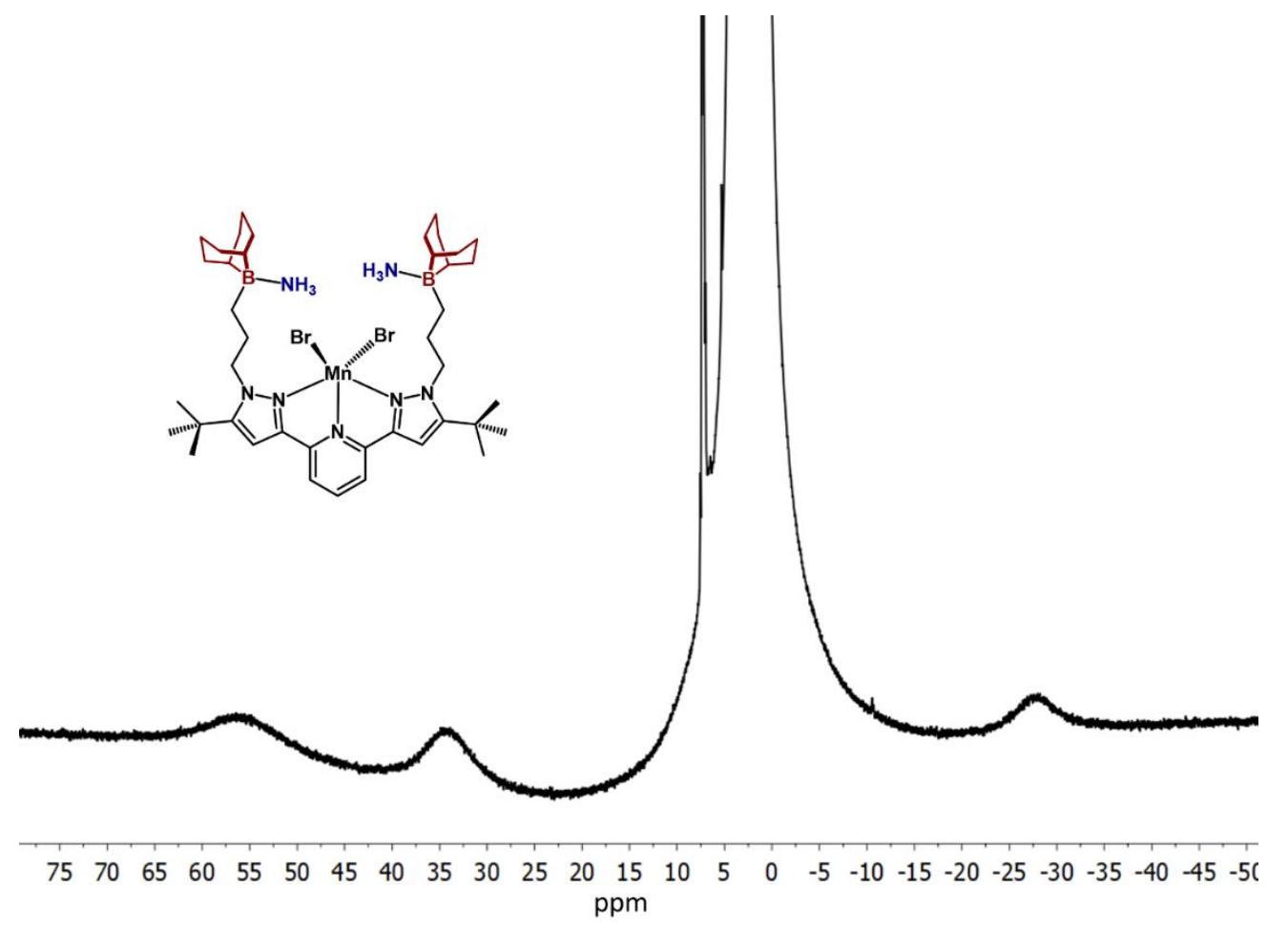

Figure $\mathrm{S5}{ }^{1} \mathrm{H} \mathrm{NMR}$ spectrum $\left(\mathrm{CDCl}_{3}, 25^{\circ} \mathrm{C}\right)$ of $\left({ }^{\mathrm{BBN} P D P}{ }^{\mathrm{tBu}}\right) \mathrm{MnBr}_{2}\left(\mathrm{NH}_{3}\right)_{2}$.

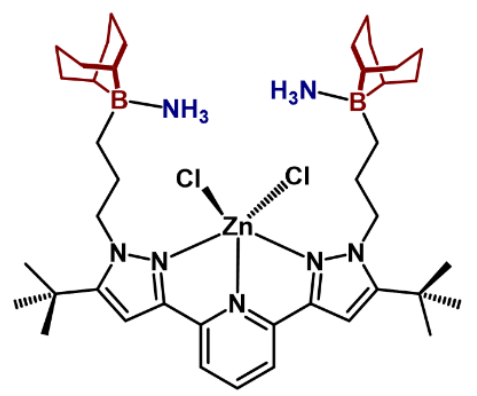

${ }^{t} \mathrm{Bu}$
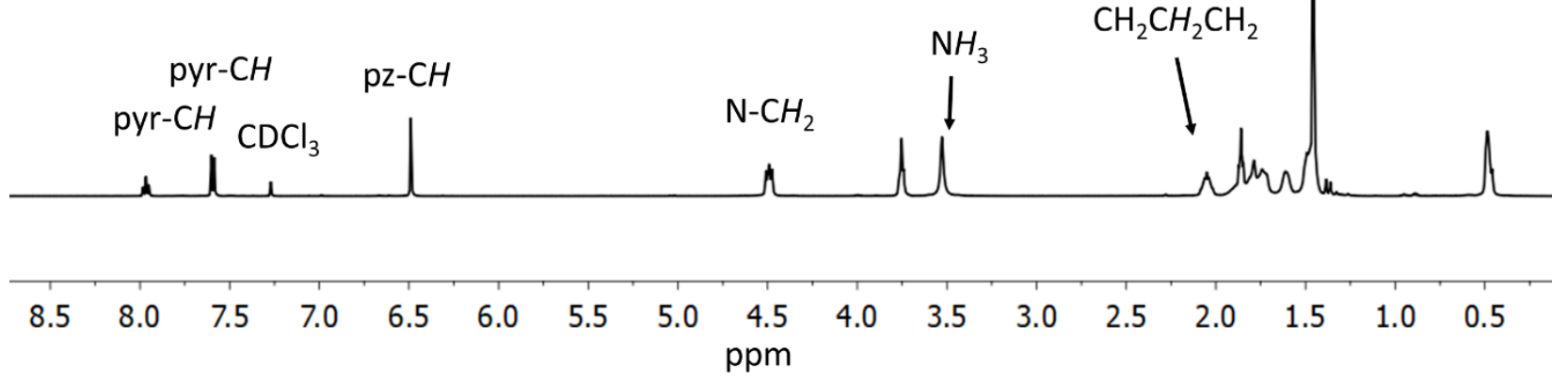

Figure $\mathbf{S 6}{ }^{1} \mathrm{H}$ NMR spectrum $\left(\mathrm{CDCl}_{3}, 25^{\circ} \mathrm{C}\right)$ of $\left({ }^{\mathrm{BBN} P D P} \mathrm{PBu}^{\mathrm{B} u}\right) \mathrm{ZnCl}_{2}\left(\mathrm{NH}_{3}\right)_{2}$. 

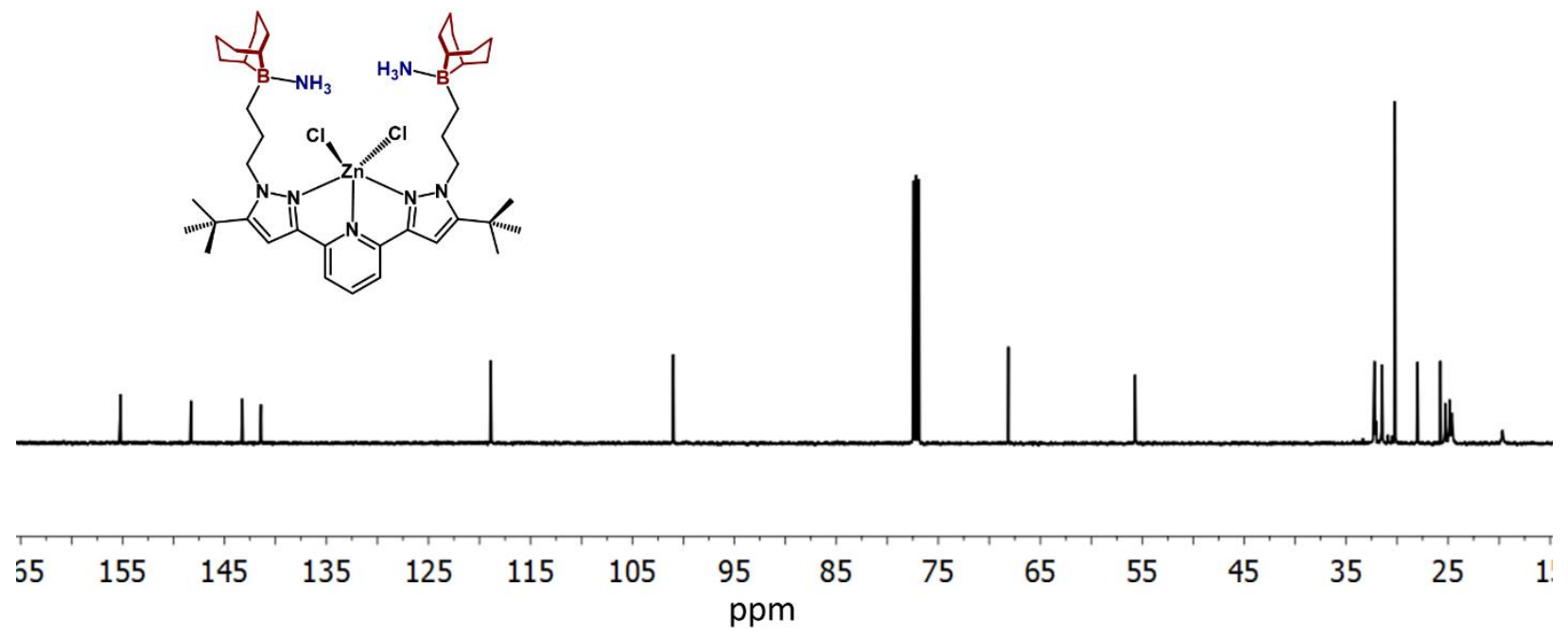

Figure $\mathrm{S7}{ }^{13} \mathrm{C}$ NMR spectrum $\left(\mathrm{CDCl}_{3}, 25^{\circ} \mathrm{C}\right)$ of $\left({ }^{\mathrm{BBN} P D P}{ }^{\mathrm{BBu}}\right) \mathrm{ZnCl}_{2}\left(\mathrm{NH}_{3}\right)_{2}$.

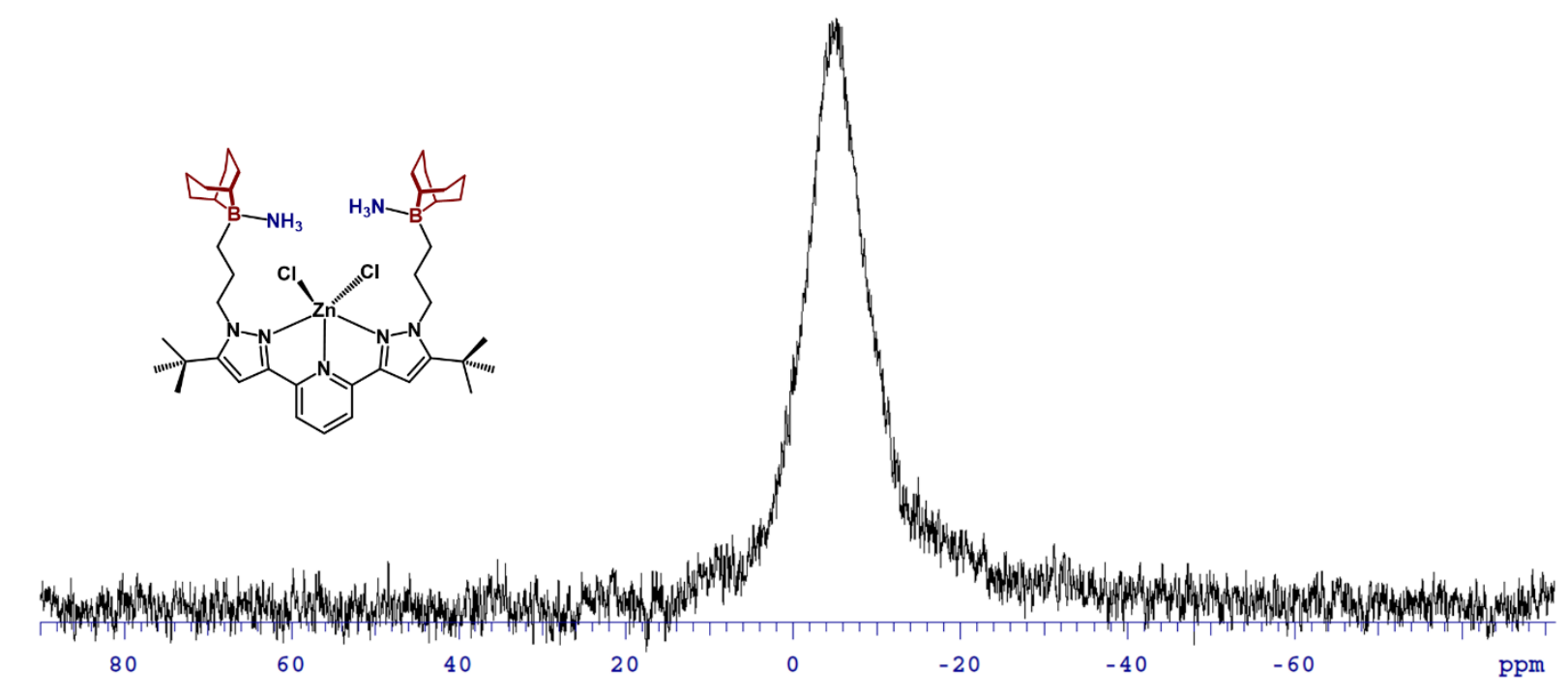

Figure $58{ }^{11} \mathrm{~B}$ NMR spectrum $\left(\mathrm{CDCl}_{3}, 25^{\circ} \mathrm{C}\right)$ of $\left({ }^{\mathrm{BBN} P D P}{ }^{\mathrm{tBu}}\right) \mathrm{ZnCl}_{2}\left(\mathrm{NH}_{3}\right)_{2}$. 


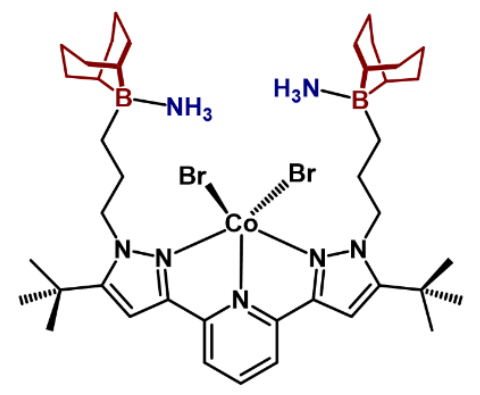

${ }^{t} \mathrm{Bu}$

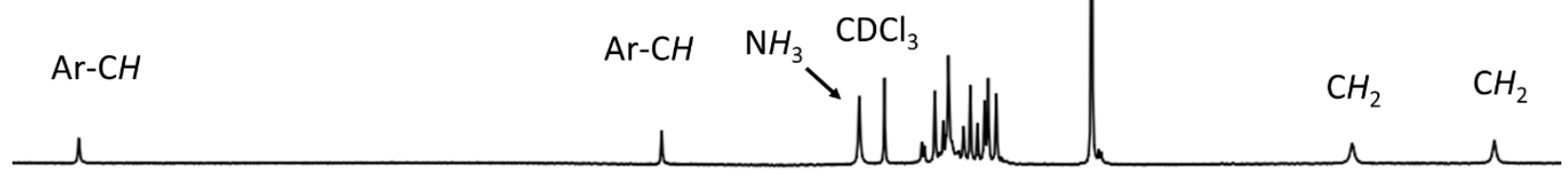

$\begin{array}{llllllllllllllllllllllllllll}80 & 75 & 70 & 65 & 60 & 55 & 50 & 45 & 40 & 35 & 30 & 25 & 20 & 15 & 10 & 5 & 0 & -5 & -10 & -15 & -20 & -25 & -30 & -35 & -40 & -45 & -50\end{array}$ ppm

Figure $S 9{ }^{1} \mathrm{H}$ NMR spectrum $\left(\mathrm{CDCl}_{3}, 25^{\circ} \mathrm{C}\right)$ of $\left({ }^{\mathrm{BBN} P D P}{ }^{\mathrm{BBu}}\right) \mathrm{CoBr}_{2}\left(\mathrm{NH}_{3}\right)_{2}$.

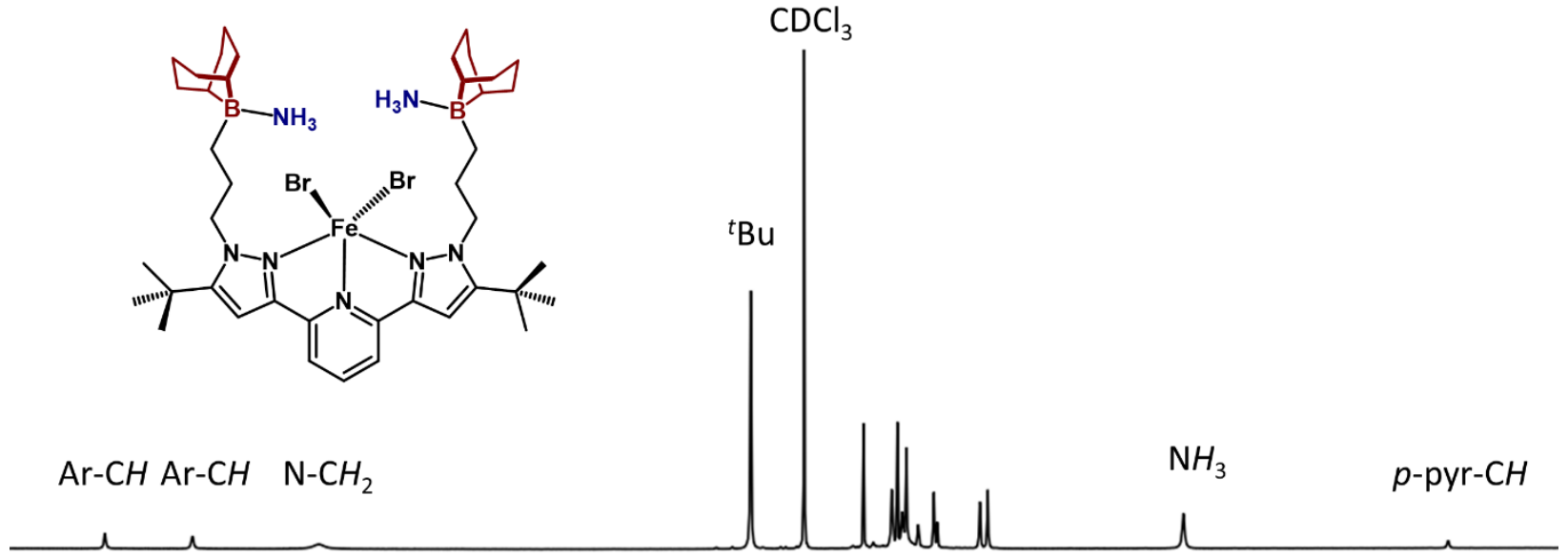

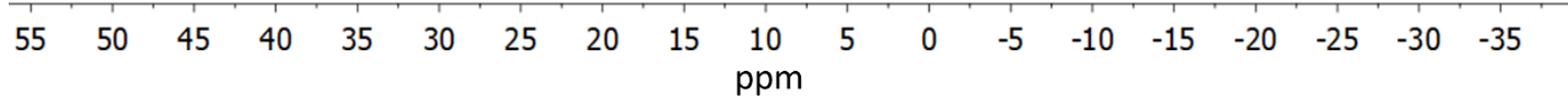

Figure $\mathrm{S} 10^{1} \mathrm{H}$ NMR spectrum $\left(\mathrm{CDCl}_{3}, 25^{\circ} \mathrm{C}\right)$ of $\left({ }^{\mathrm{BBN} P D P}{ }^{\mathrm{BB} u}\right) \mathrm{FeBr}_{2}\left(\mathrm{NH}_{3}\right)_{2}$. 

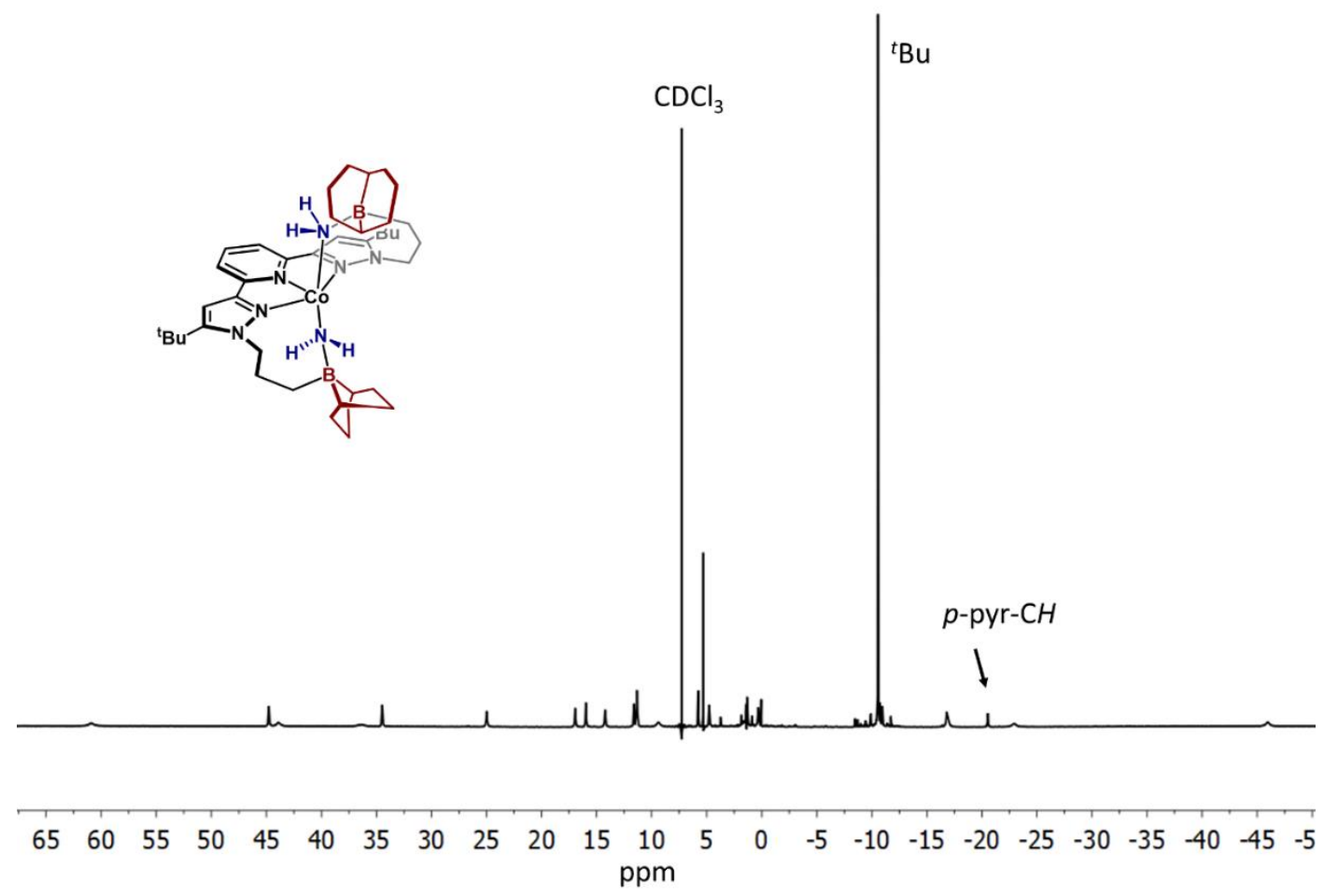

Figure $\mathrm{S} 11{ }^{1} \mathrm{H}$ NMR spectrum $\left(\mathrm{CDCl}_{3}, 25^{\circ} \mathrm{C}\right)$ of $\left({ }^{\mathrm{BBN} P D P}{ }^{t \mathrm{Bu}}\right) \mathrm{Co}\left(\mathrm{NH}_{2}\right)_{2}$.
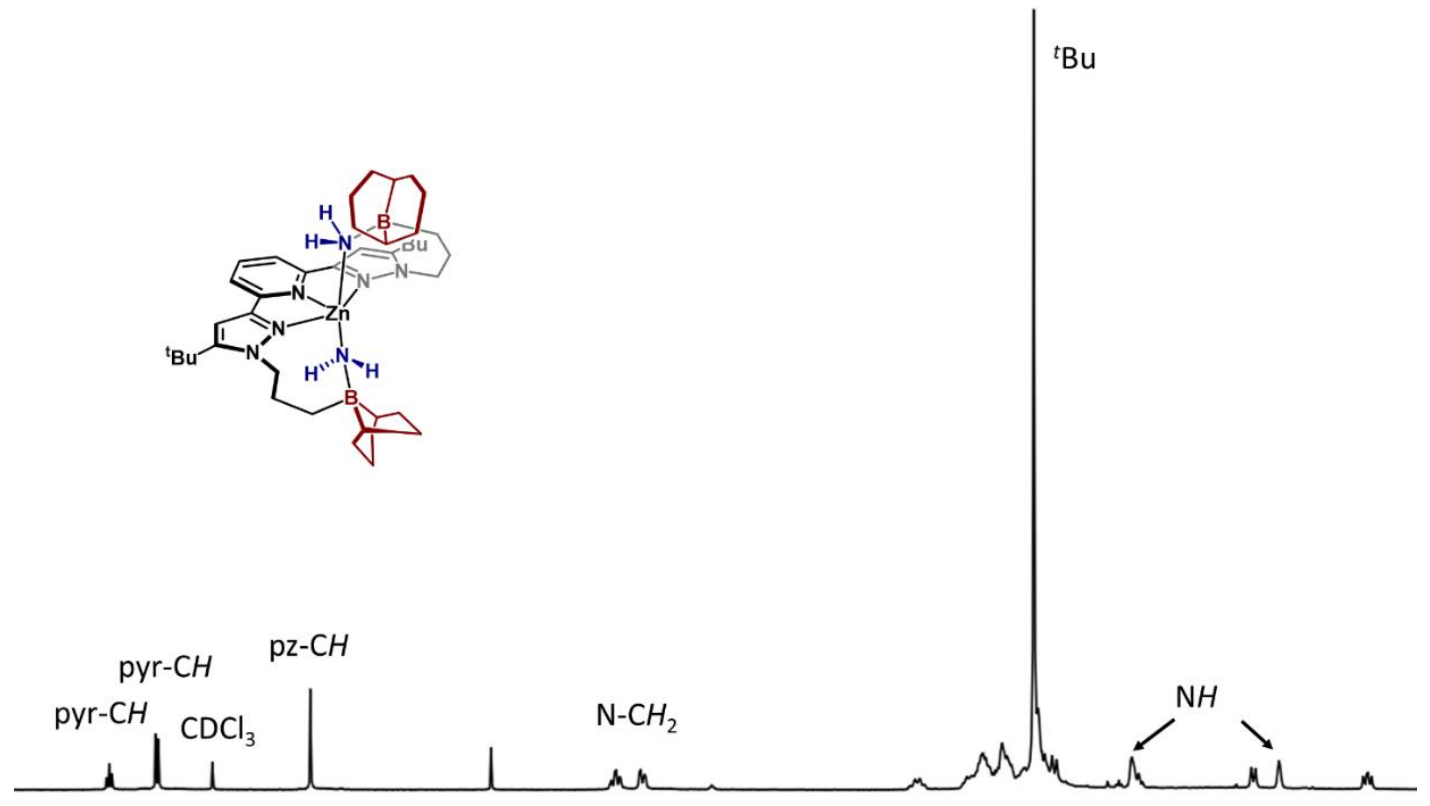

$\begin{array}{llllllllllllllllllllll}8.5 & 8.0 & 7.5 & 7.0 & 6.5 & 6.0 & 5.5 & 5.0 & 4.5 & \begin{array}{l}4.0 \\ \mathrm{ppm}\end{array} & 3.5 & 3.0 & 2.5 & 2.0 & 1.5 & 1.0 & 0.5 & 0.0 & -0.5 & -1.0\end{array}$

Figure $\mathrm{S} 12{ }^{1} \mathrm{H}$ NMR spectrum $\left(\mathrm{CDCl}_{3}, 25^{\circ} \mathrm{C}\right)$ of $\left({ }^{\mathrm{BBN} P D P}{ }^{\mathrm{Bu} u}\right) \mathrm{Zn}\left(\mathrm{NH}_{2}\right)_{2}$. 

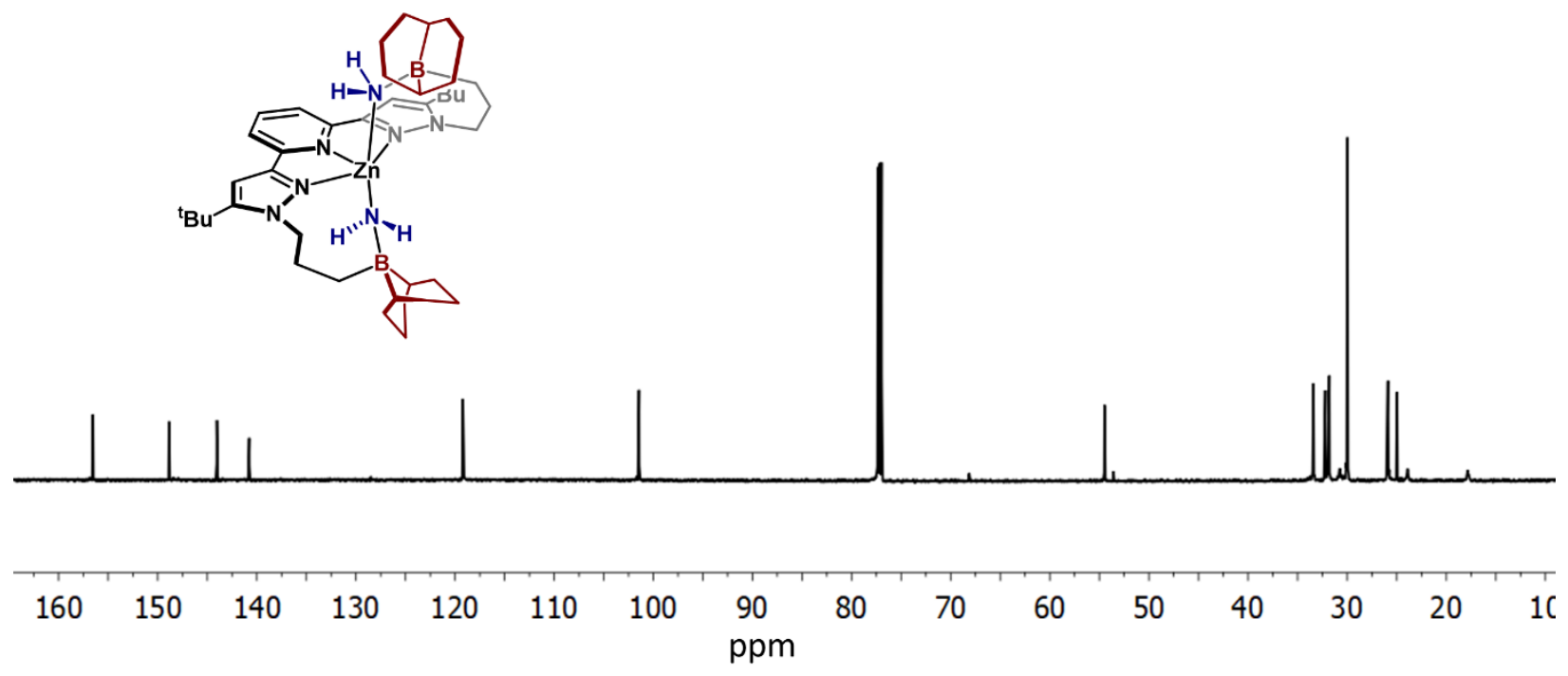

Figure $\mathrm{S} 13{ }^{13} \mathrm{C}$ NMR spectrum $\left(\mathrm{CDCl}_{3}, 25^{\circ} \mathrm{C}\right)$ of $\left({ }^{\mathrm{BBN} P D P}{ }^{\mathrm{tBu}}\right) \mathrm{Zn}\left(\mathrm{NH}_{2}\right)_{2}$.

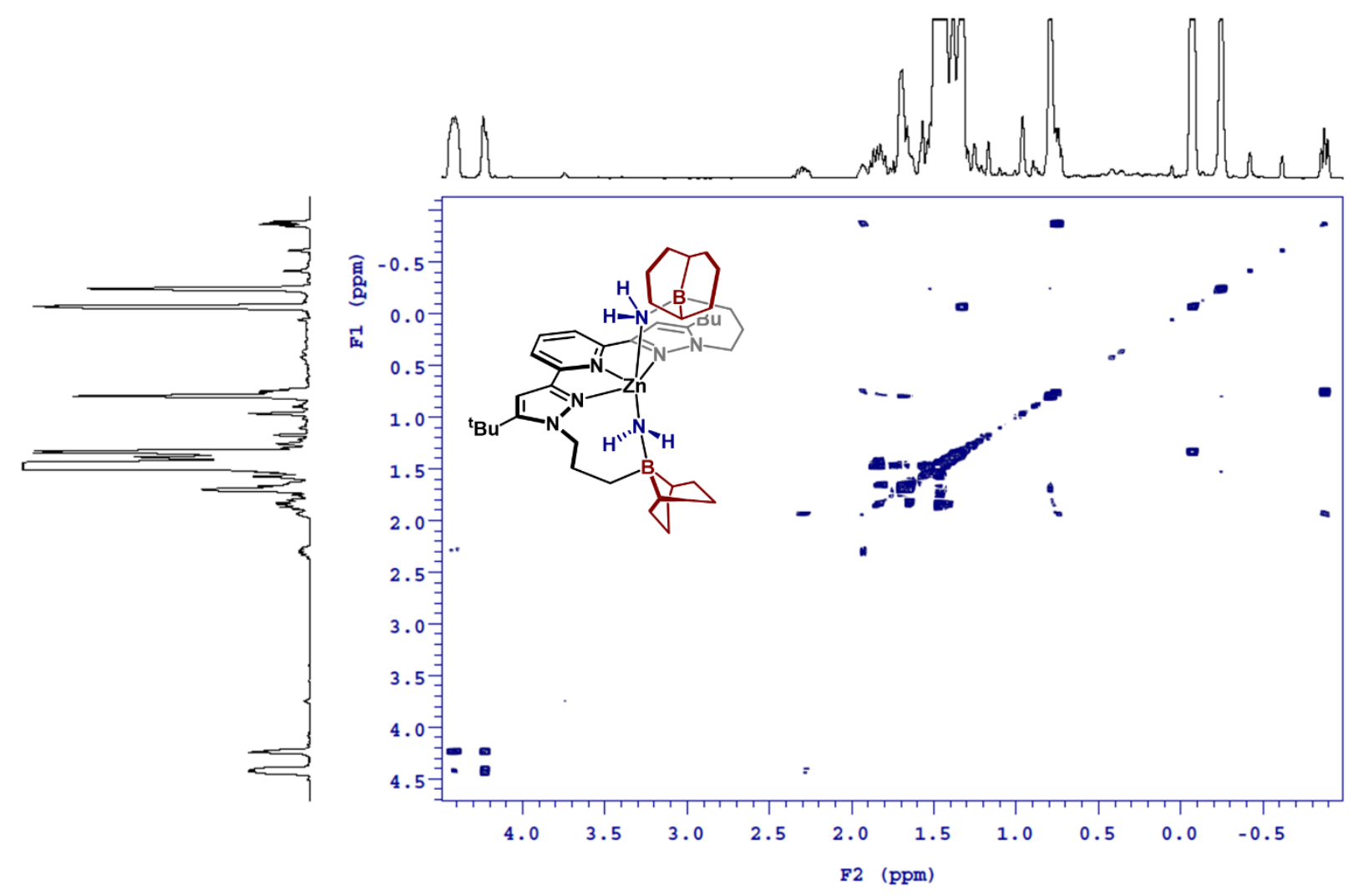

Figure $\mathrm{S} 14{ }^{1} \mathrm{H}^{-1} \mathrm{H}$ COSY spectrum $\left(\mathrm{CDCl}_{3}, 25^{\circ} \mathrm{C}\right)$ of $\left({ }^{\text {BBN PDP }}{ }^{\mathrm{tBu}}\right) \mathrm{Zn}\left(\mathrm{NH}_{2}\right)_{2}$. 

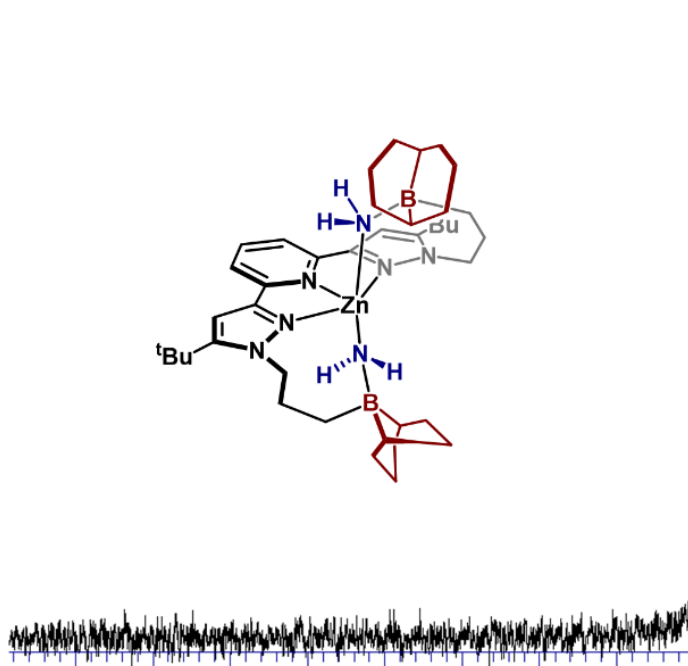

80

60

40

20

$-20$

$-40$

$-60$

$-80$

$-100$

ppm

Figure $\mathbf{S} 15^{11} \mathrm{~B}$ NMR spectrum $\left(\mathrm{CDCl}_{3}, 25^{\circ} \mathrm{C}\right)$ of $\left({ }^{\mathrm{BBN}} \mathrm{PDP}{ }^{\mathrm{BBu}}\right) \mathrm{Zn}\left(\mathrm{NH}_{2}\right)_{2}$.

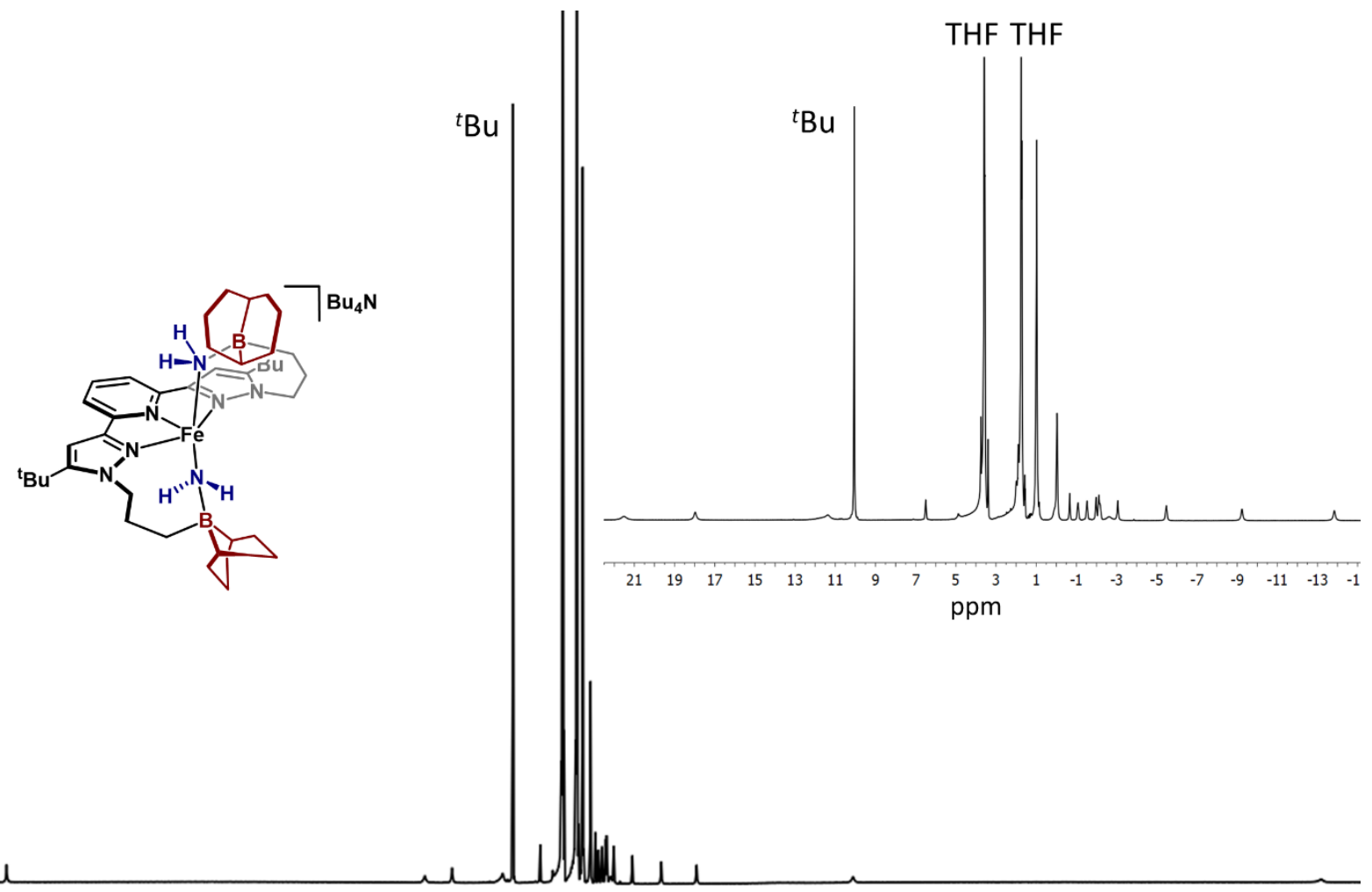

$\begin{array}{llllllllllllllllllllll}80 & 70 & 60 & 50 & 40 & 30 & 20 & 10 & 0 & -10 & -20 & -30 & -40 & -50 & -60 & -70 & -80 & -90 & -11 \\ \mathrm{ppm}\end{array}$

Figure $\mathrm{S} 16{ }^{1} \mathrm{H}$ NMR spectrum $\left(\mathrm{THF}, 25^{\circ} \mathrm{C}\right)$ of $\left[\mathrm{Bu}_{4} \mathrm{~N}\right]\left[\left({ }^{\mathrm{BBN} P D P}{ }^{\mathrm{BBu}}\right) \mathrm{Fe}\left(\mathrm{NH}_{2}\right)_{2}\right]$. 


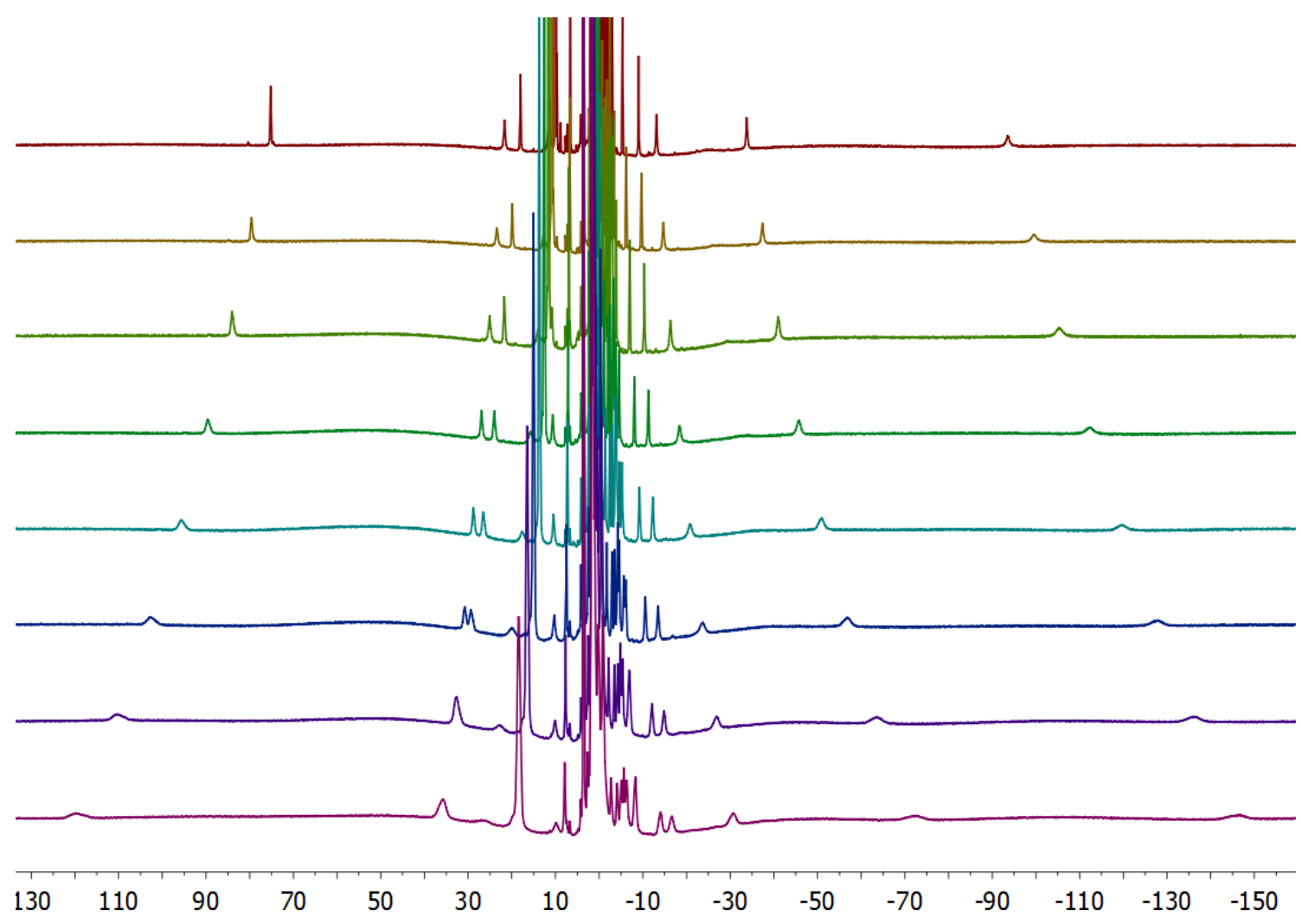

Figure S17 Variable temperature ${ }^{1} \mathrm{H}$ NMR spectra $\left(\mathrm{ppm}\right.$, THF- $\left.d_{8}, 193-298 \mathrm{~K}\right)$ of $\left[\mathrm{Bu}_{4} \mathrm{~N}\right]\left[\left({ }^{(B \mathrm{BN}} \mathrm{PDP}{ }^{\mathrm{tBu}}\right) \mathrm{Fe}\left(\mathrm{NH}_{2}\right)_{2}\right]$.

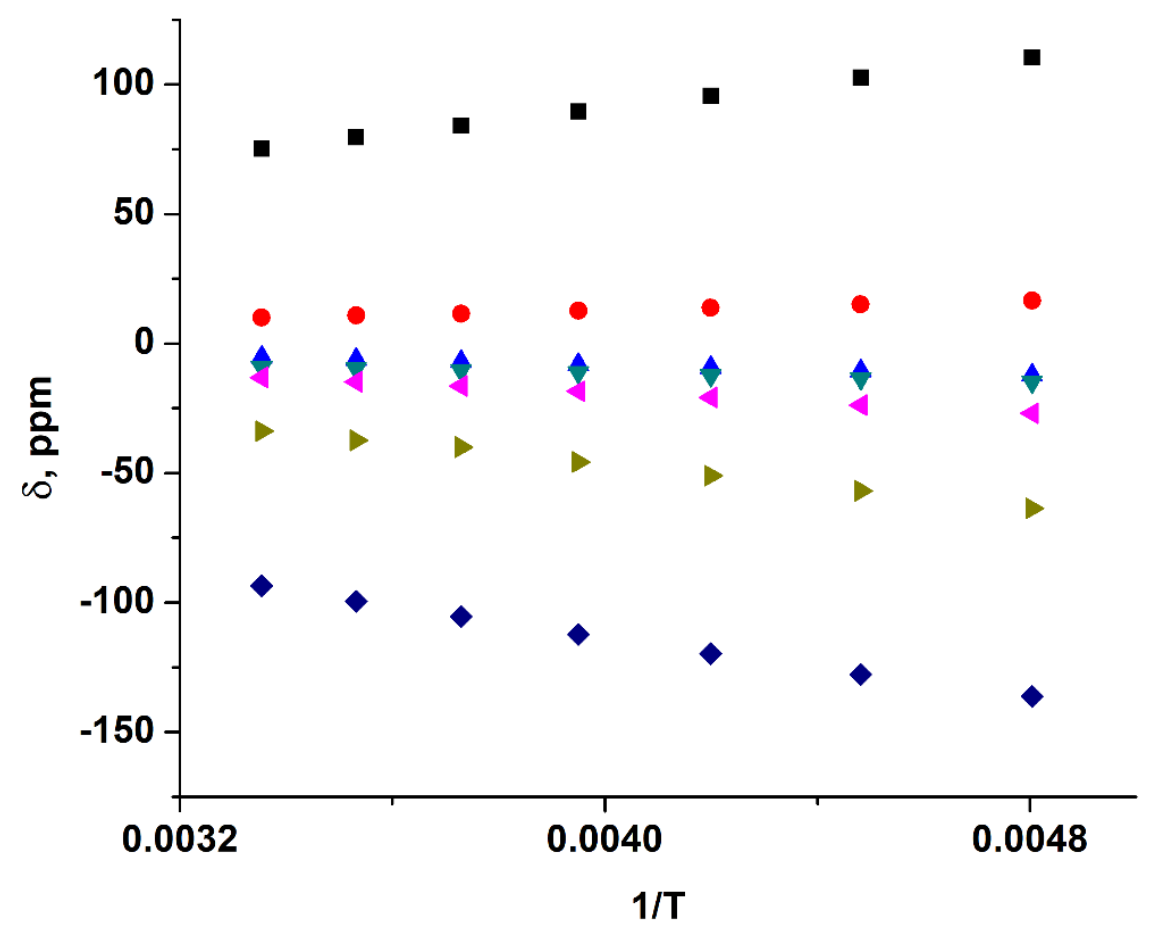

Figure S18 Plot of chemical shift vs. 1/T derived from variable temperature ${ }^{1} \mathrm{H}$ NMR spectra of $\left[\mathrm{Bu}_{4} \mathrm{~N}\right]\left[\left({ }^{\mathrm{BBN} P D P}{ }^{\mathrm{tBu}}\right) \mathrm{Fe}\left(\mathrm{NH}_{2}\right)_{2}\right]$. 


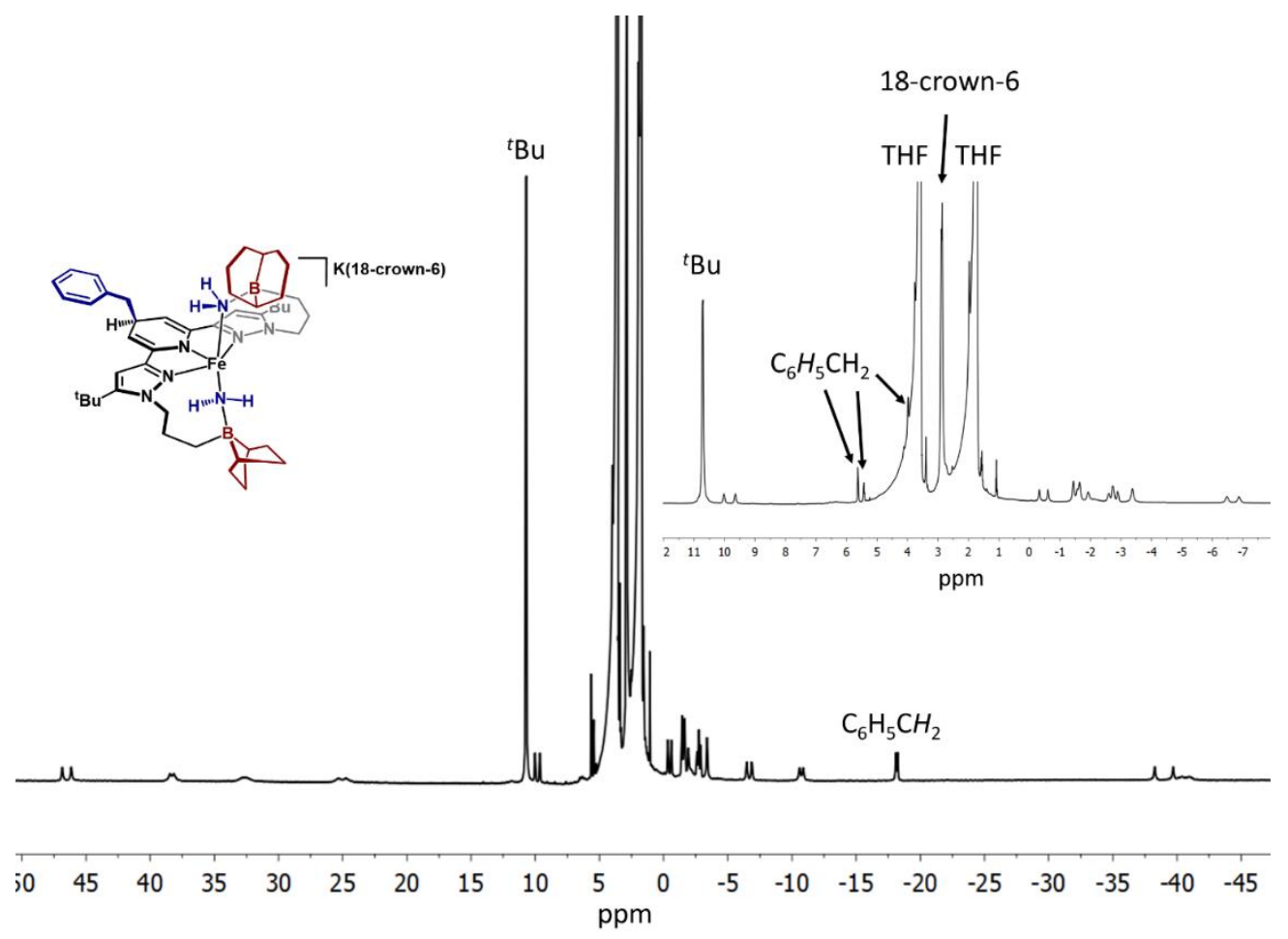

Figure $\mathrm{S} 19{ }^{1} \mathrm{H}$ NMR spectrum $\left(\mathrm{CDCl}_{3}, 25^{\circ} \mathrm{C}\right)$ of $[\mathrm{K}(18-\mathrm{crown}-6)]\left[\left(\right.\right.$ benzyl- $\left.\left.{ }^{\mathrm{BBN}} \mathrm{PDP}{ }^{\mathrm{tBu}}\right) \mathrm{Fe}\left(\mathrm{NH}_{2}\right)_{2}\right]$.

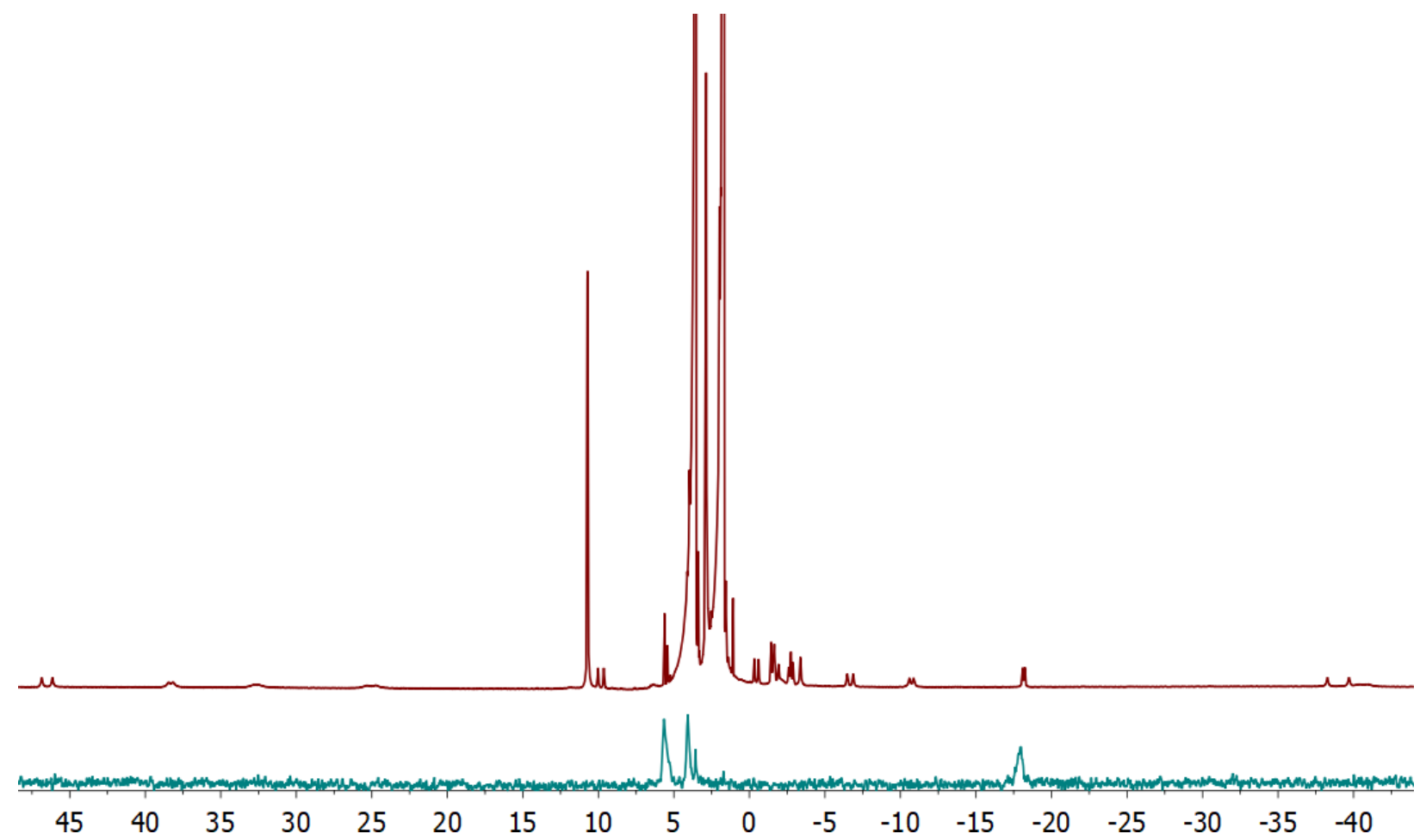

Figure S2O Overlay of ${ }^{1} \mathrm{H}$ and ${ }^{2} \mathrm{H}$ NMR spectra (THF, $\left.25^{\circ} \mathrm{C}\right)$ of $\left[\mathrm{K}(2,2,2\right.$-cryptand) $]\left[\left(\mathrm{C}_{6} \mathrm{H}_{5} \mathrm{CH}_{2}\right.\right.$ $\left.\left.{ }^{B B N} P D P^{t B u}\right) \mathrm{Fe}\left(\mathrm{NH}_{2}\right)_{2}\right]$ and $[K(2,2,2$-cryptand $)]\left[\left(\mathrm{C}_{6} \mathrm{D}_{5} \mathrm{CD}_{2}{ }^{-{ }^{B B N}}{ }^{\circ D P P}{ }^{t B u}\right) \mathrm{Fe}\left(\mathrm{NH}_{2}\right)_{2}\right]$. 


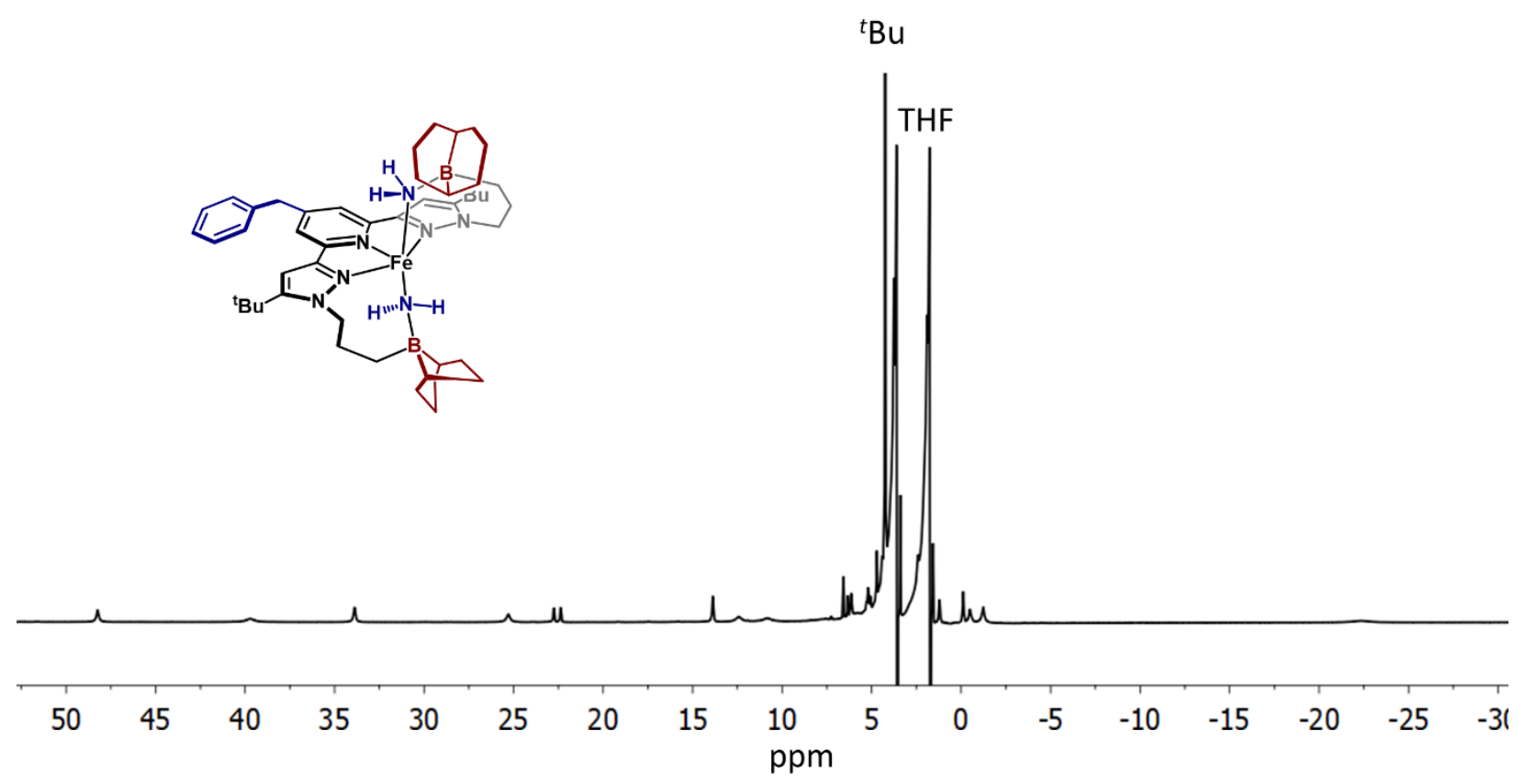

Figure S21 ${ }^{1} \mathrm{H}$ NMR spectrum (THF, $\left.25{ }^{\circ} \mathrm{C}\right)$ of $\left(\mathrm{C}_{6} \mathrm{H}_{5} \mathrm{CH}_{2^{-}}{ }^{\mathrm{BBN}} \mathrm{PDP} \mathrm{P}^{\mathrm{tBu}}\right) \mathrm{Fe}\left(\mathrm{NH}_{2}\right)_{2}$.

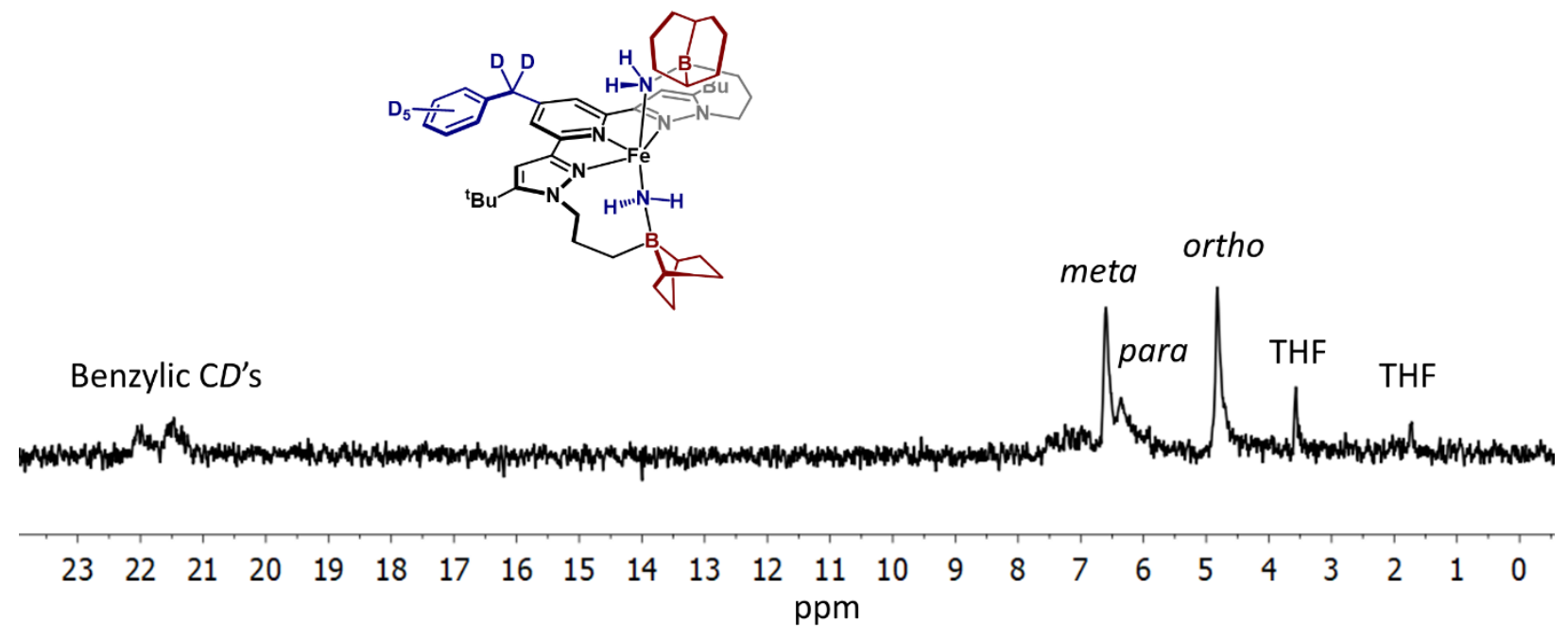

Figure S22 ${ }^{2} \mathrm{H}$ NMR spectrum $\left(\mathrm{THF}, 25^{\circ} \mathrm{C}\right)$ of $\left(\mathrm{C}_{6} \mathrm{D}_{5} \mathrm{CD}_{2^{-}}{ }^{\mathrm{BBN}} \mathrm{PDP} \mathrm{P}^{\mathrm{BBu}}\right) \mathrm{Fe}\left(\mathrm{NH}_{2}\right)_{2}$.

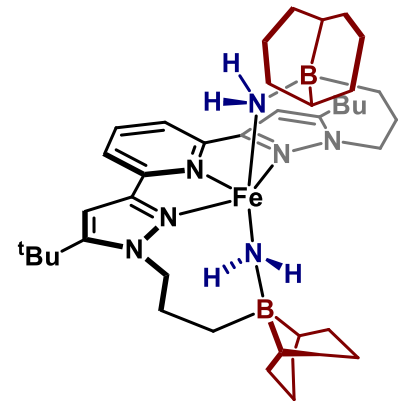

\begin{tabular}{l|l} 
solvent & soluble? \\
\hline Benzene & Insoluble \\
$0-\mathrm{C}_{6} \mathrm{H}_{4} \mathrm{Cl}_{2}$ & Soluble \\
$\mathrm{O}_{6}-\mathrm{C}_{6} \mathrm{H}_{4} \mathrm{~F}_{2}$ & Insoluble \\
$\mathrm{C}_{6} \mathrm{H}_{5} \mathrm{~F}$ & Insoluble \\
$\mathrm{MeNO}_{2}$ & Decomposed \\
Acetone & Decomposed \\
THF & Soluble \\
DMF & Soluble \\
DMA & Soluble \\
$\mathrm{CH}_{2} \mathrm{Cl}_{2}$ & Soluble
\end{tabular}

Figure S23 Summary of solubility of ( $\left.{ }^{B B N} \mathrm{PDP}{ }^{\mathrm{tBu}}\right) \mathrm{Fe}\left(\mathrm{NH}_{2}\right)_{2}$. 

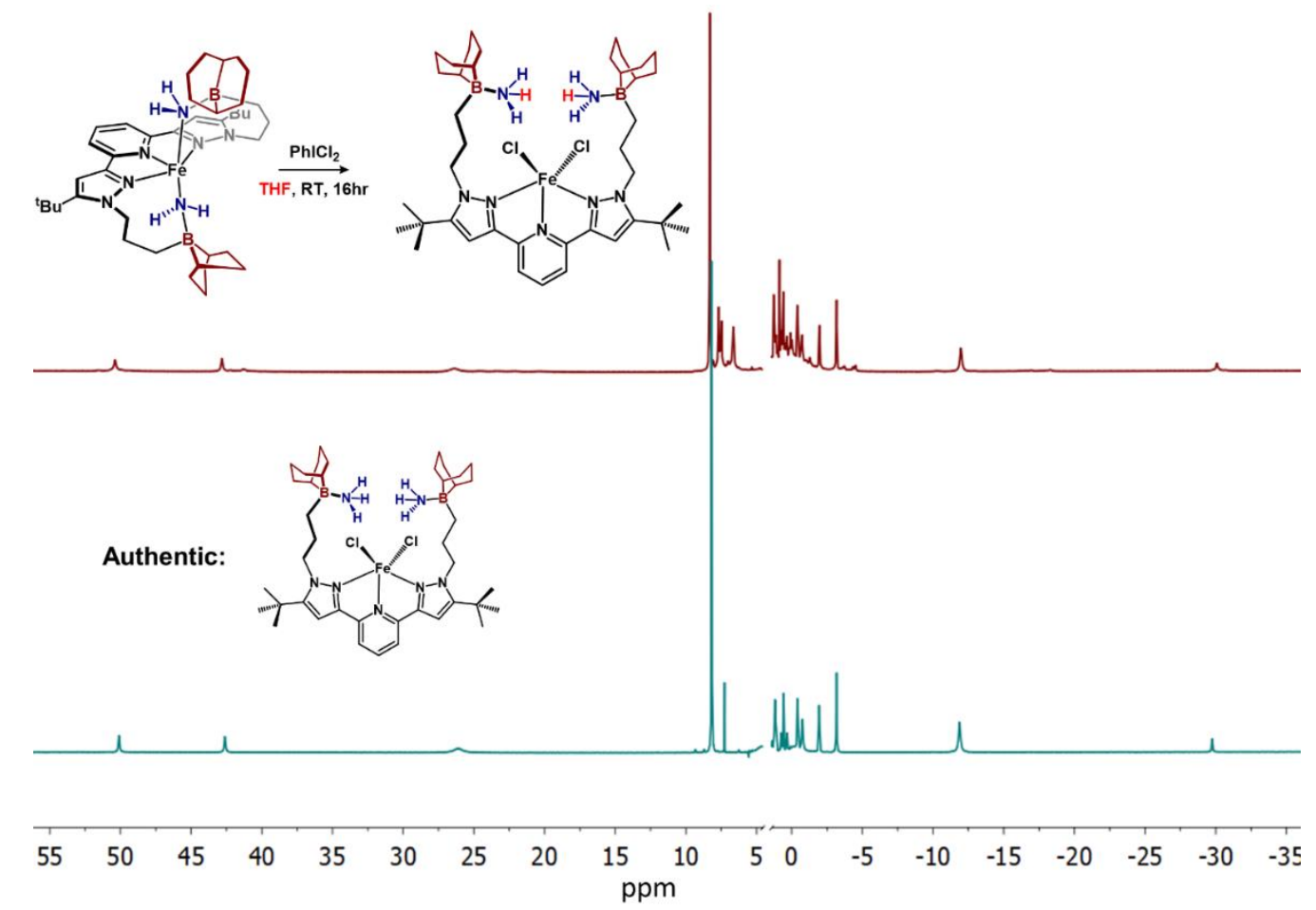

Figure S24 Top: ${ }^{1} \mathrm{H}$ NMR spectrum $\left(\mathrm{THF}, 25^{\circ} \mathrm{C}\right)$ of crude material obtained from oxidation of $\left({ }^{\text {BBNPDP }}{ }^{\mathrm{tBu}}\right) \mathrm{Fe}\left(\mathrm{NH}_{2}\right)_{2}$ with $\mathrm{PhICl}_{2}$ in THF. Bottom: ${ }^{1} \mathrm{H}$ NMR spectrum $\left(\mathrm{THF}, 25^{\circ} \mathrm{C}\right)$ of authentic sample of $\left({ }^{\mathrm{BBN}} \mathrm{PDP}{ }^{\mathrm{tBu}}\right) \mathrm{FeCl}_{2}\left(\mathrm{NH}_{3}\right)_{2}$. The THF solvent resonances are omitted to increase clarity.
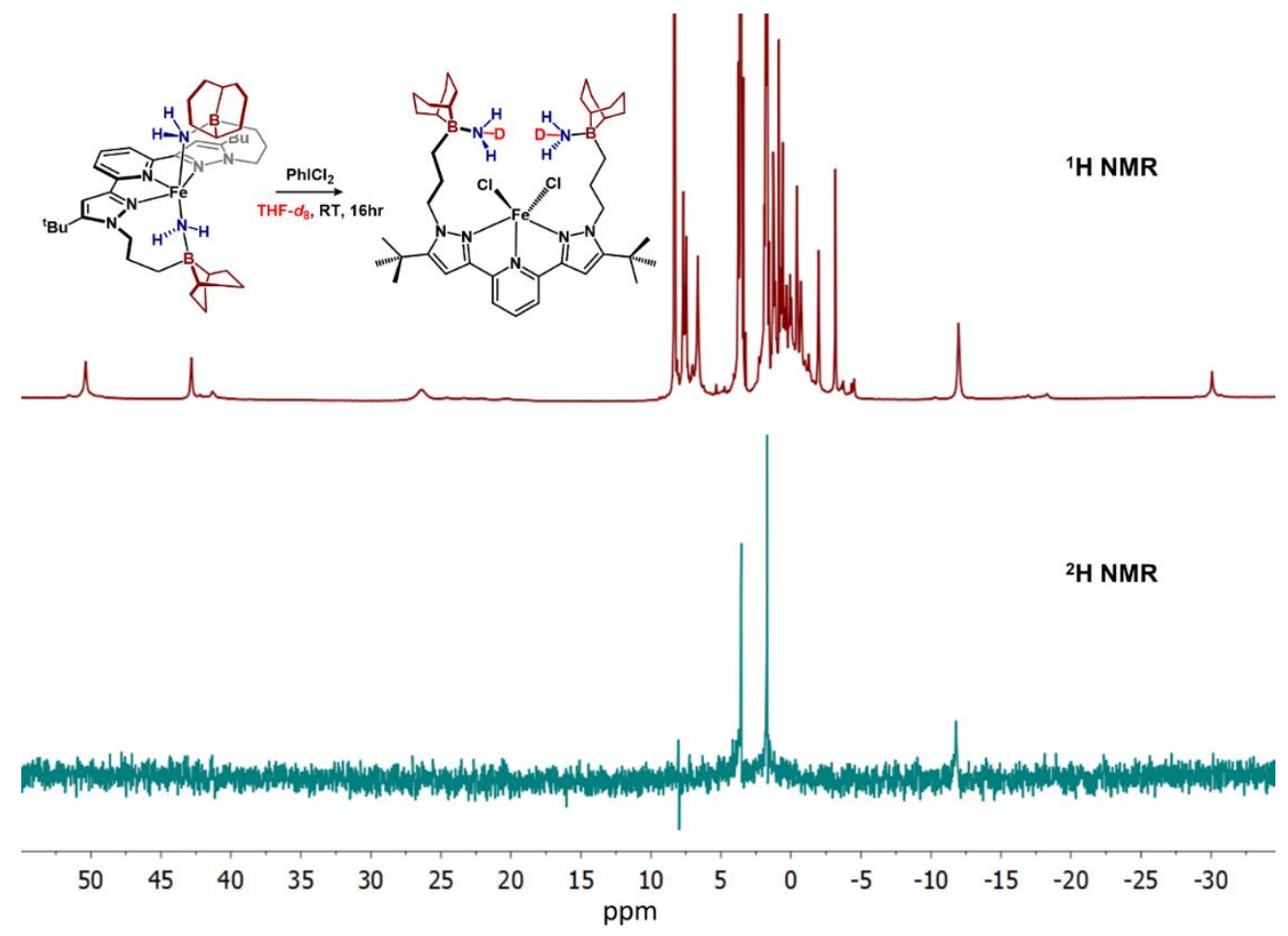

Figure S25 Top: ${ }^{1} \mathrm{H}$ NMR spectrum $\left(\mathrm{THF}, 25^{\circ} \mathrm{C}\right.$ ) of crude material obtained from oxidation of $\left({ }^{\mathrm{BBN}} \mathrm{PDP}{ }^{\mathrm{tBu}}\right) \mathrm{Fe}\left(\mathrm{NH}_{2}\right)_{2}$ with $\mathrm{PhICl}_{2}$ in THF- $d_{8}$. Bottom: ${ }^{2} \mathrm{H} \mathrm{NMR}$ spectrum $\left(\mathrm{THF}, 25^{\circ} \mathrm{C}\right)$ of same mixture. 


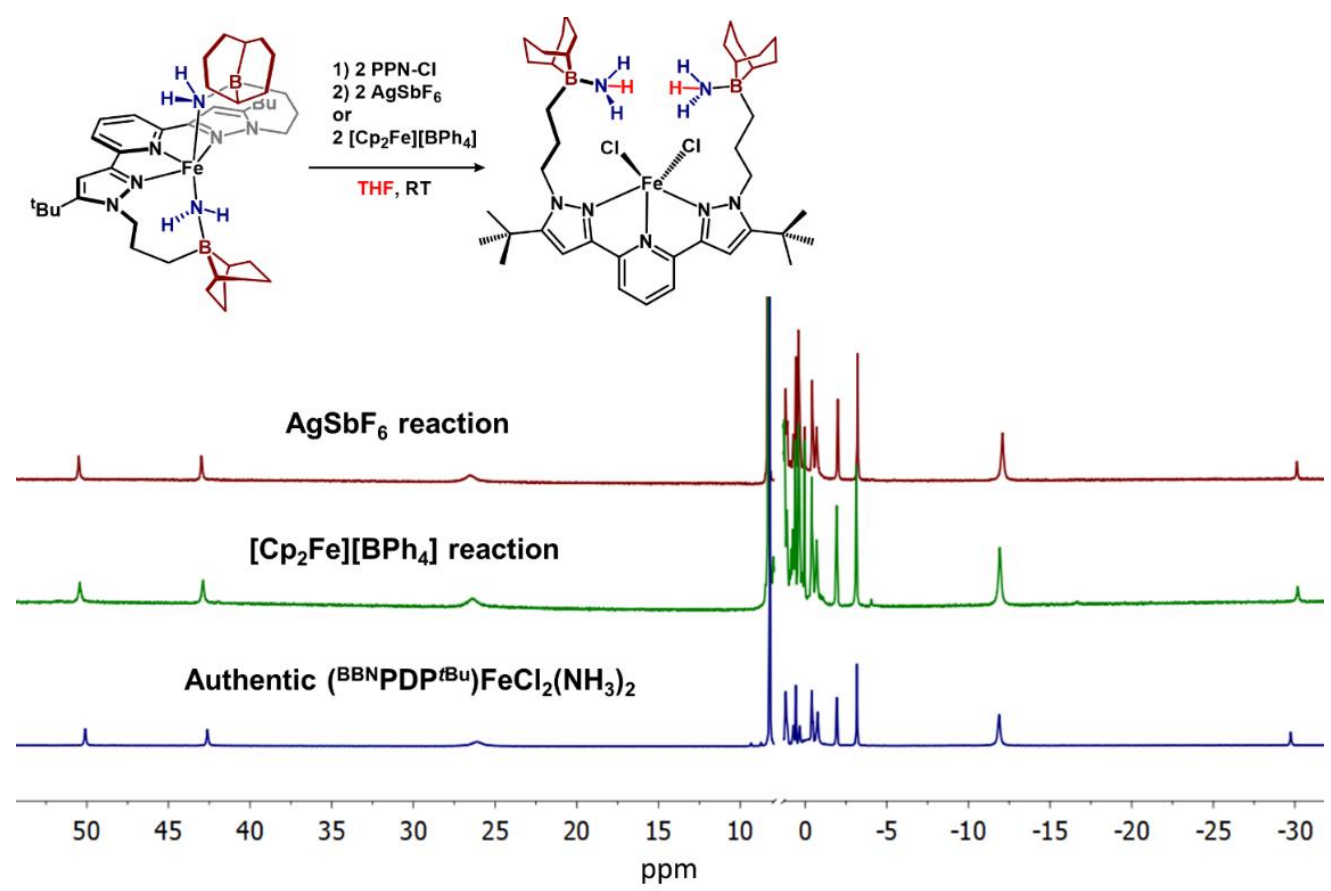

Figure S26 Top: ${ }^{1} \mathrm{H}$ NMR spectrum $\left(\mathrm{THF}, 25^{\circ} \mathrm{C}\right.$ ) of crude material obtained from oxidation of $\left({ }^{B B N} \mathrm{PDP}{ }^{t B u}\right) \mathrm{Fe}\left(\mathrm{NH}_{2}\right)_{2}$ with $\mathrm{AgSbF}_{6}$ in the presence of PPN-Cl in THF. Middle: ${ }^{1} \mathrm{H}$ NMR spectrum $\left(\mathrm{THF}, 25^{\circ} \mathrm{C}\right)$ of crude material obtained from oxidation of $\left({ }^{\mathrm{BBN} P D P}{ }^{\mathrm{BBu}}\right) \mathrm{Fe}\left(\mathrm{NH}_{2}\right)_{2}$ with $\left[\mathrm{Cp}_{2} \mathrm{Fe}\right]\left[\mathrm{BPh}_{4}\right]$ in the presence of PPN-Cl in THF. Bottom: ${ }^{1} \mathrm{H}$ NMR spectrum $\left(\mathrm{THF}, 25^{\circ} \mathrm{C}\right.$ ) of authentic sample of ( $\left.{ }^{\mathrm{BBN} P D P} \mathrm{BBu}^{\mathrm{Bu}}\right) \mathrm{FeCl}_{2}\left(\mathrm{NH}_{3}\right)_{2}$. The THF solvent resonances are omitted to increase clarity.

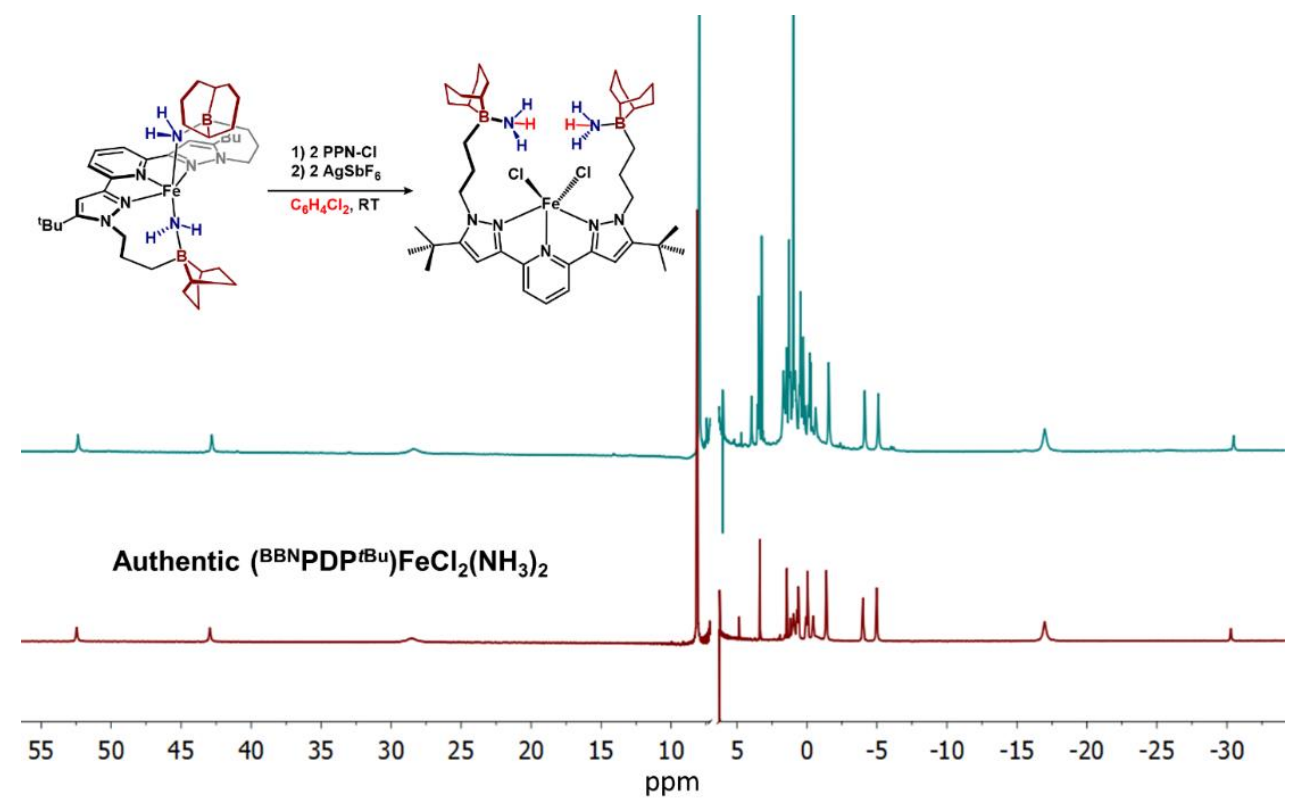

Figure S27 Top: ${ }^{1} \mathrm{H}$ NMR spectrum $\left(o-\mathrm{C}_{6} \mathrm{H}_{4} \mathrm{Cl}_{2}, 25^{\circ} \mathrm{C}\right)$ of crude material obtained from oxidation of $\left({ }^{B B N} \mathrm{PDP}{ }^{\mathrm{tBu}}\right) \mathrm{Fe}\left(\mathrm{NH}_{2}\right)_{2}$ with $\mathrm{AgSbF}_{6}$ in the presence of PPN-Cl in $o_{-}-\mathrm{C}_{6} \mathrm{H}_{4} \mathrm{Cl}_{2}$. Bottom: ${ }^{1} \mathrm{H}$ NMR spectrum (o$\left.\mathrm{C}_{6} \mathrm{H}_{4} \mathrm{Cl}_{2}, 25^{\circ} \mathrm{C}\right)$ of authentic sample of $\left({ }^{\mathrm{BBN}} \mathrm{PDP}{ }^{\mathrm{tBu}}\right) \mathrm{FeCl}_{2}\left(\mathrm{NH}_{3}\right)_{2}$. The $o-\mathrm{C}_{6} \mathrm{H}_{4} \mathrm{Cl}_{2}$ solvent resonances are omitted to increase clarity. 


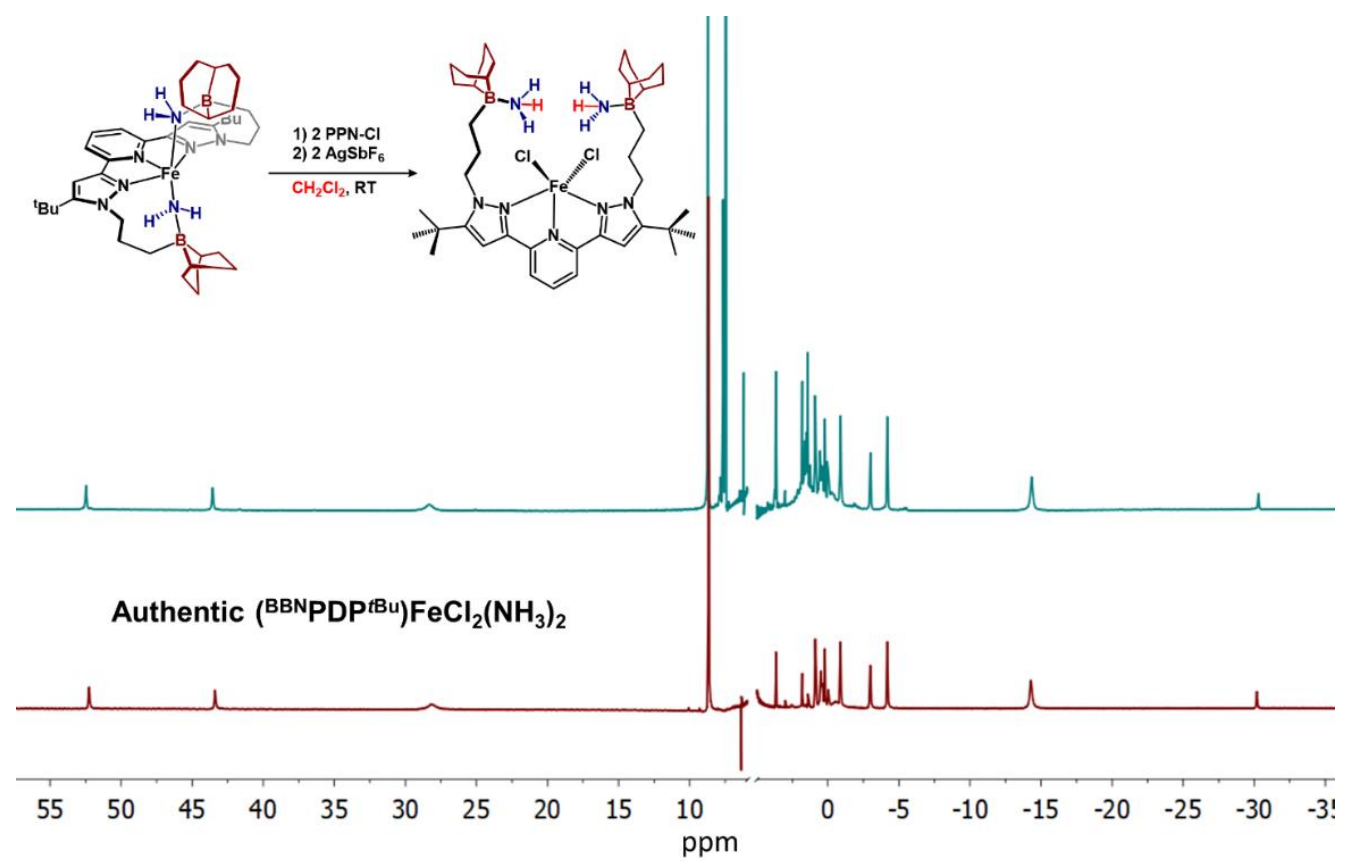

Figure S28 Top: ${ }^{1} \mathrm{H}$ NMR spectrum $\left(\mathrm{CH}_{2} \mathrm{Cl}_{2}, 25^{\circ} \mathrm{C}\right)$ of crude material obtained from oxidation of $\left({ }^{B B N} \mathrm{PDP}{ }^{\mathrm{tBu}}\right) \mathrm{Fe}\left(\mathrm{NH}_{2}\right)_{2}$ with $\mathrm{AgSbF}$ in the presence of $\mathrm{PPN}-\mathrm{Cl}$ in $\mathrm{CH}_{2} \mathrm{Cl}_{2}$. Bottom: ${ }^{1} \mathrm{H} \mathrm{NMR}$ spectrum $\left(\mathrm{CH}_{2} \mathrm{Cl}_{2}\right.$, $25^{\circ} \mathrm{C}$ ) of authentic sample of $\left({ }^{\mathrm{BBN}} \mathrm{PDP}{ }^{\mathrm{tBu}}\right) \mathrm{FeCl}_{2}\left(\mathrm{NH}_{3}\right)_{2}$. The $\mathrm{CH}_{2} \mathrm{Cl}_{2}$ solvent resonances are omitted to increase clarity.

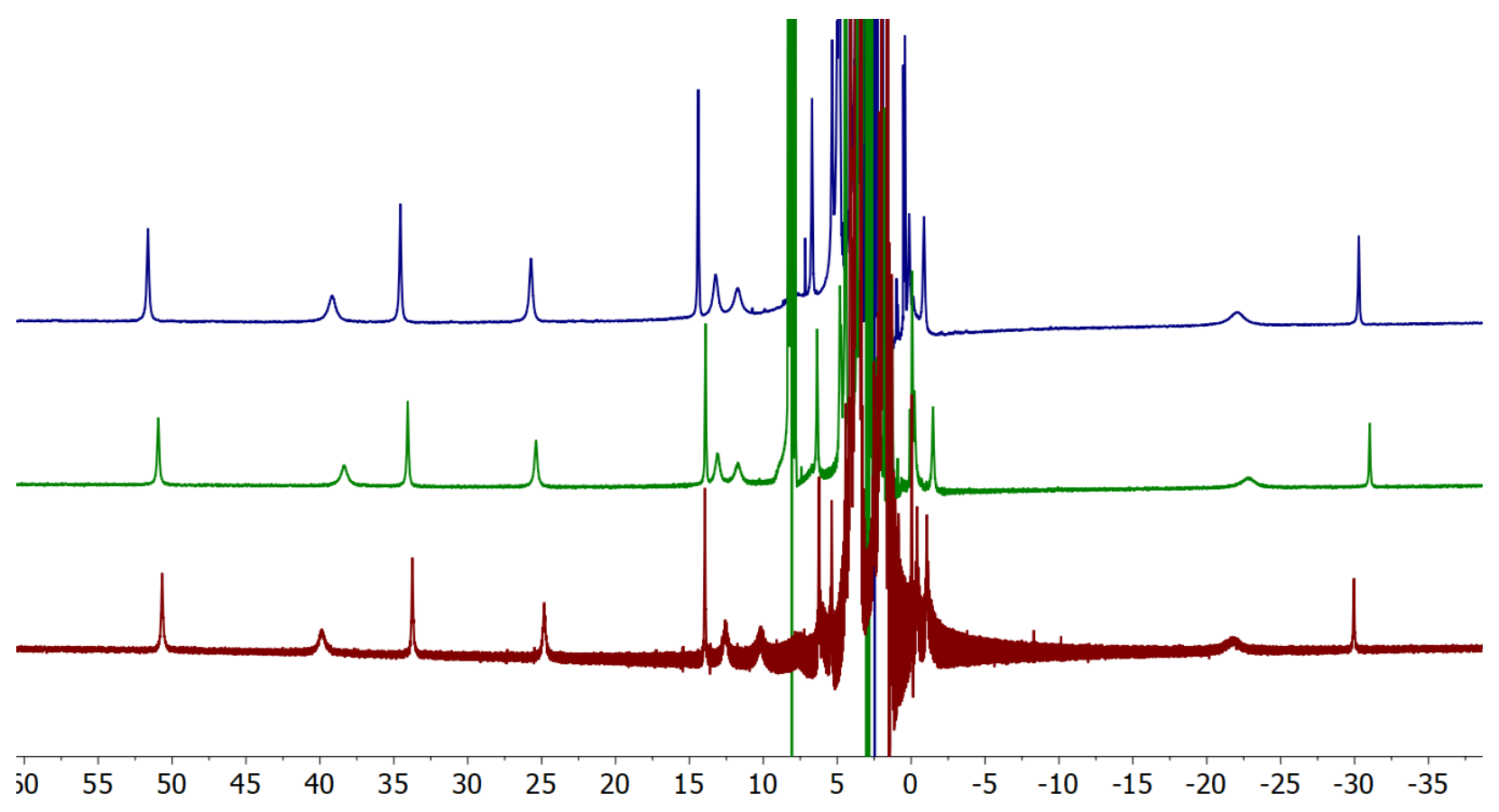

Figure S29 ${ }^{1} \mathrm{H}$ NMR spectra $\left(25^{\circ} \mathrm{C}\right)$ of $\left({ }^{\mathrm{BBN} P D P} \mathrm{P}^{\mathrm{BBu}}\right) \mathrm{Fe}\left(\mathrm{NH}_{2}\right)_{2}$ in $\mathrm{N}, \mathrm{N}$-dimethylacetamide (top), $\mathrm{N}, \mathrm{N}$ dimethylformamide (middle), and tetrahydrofuran (bottom). X-axis units in ppm. 


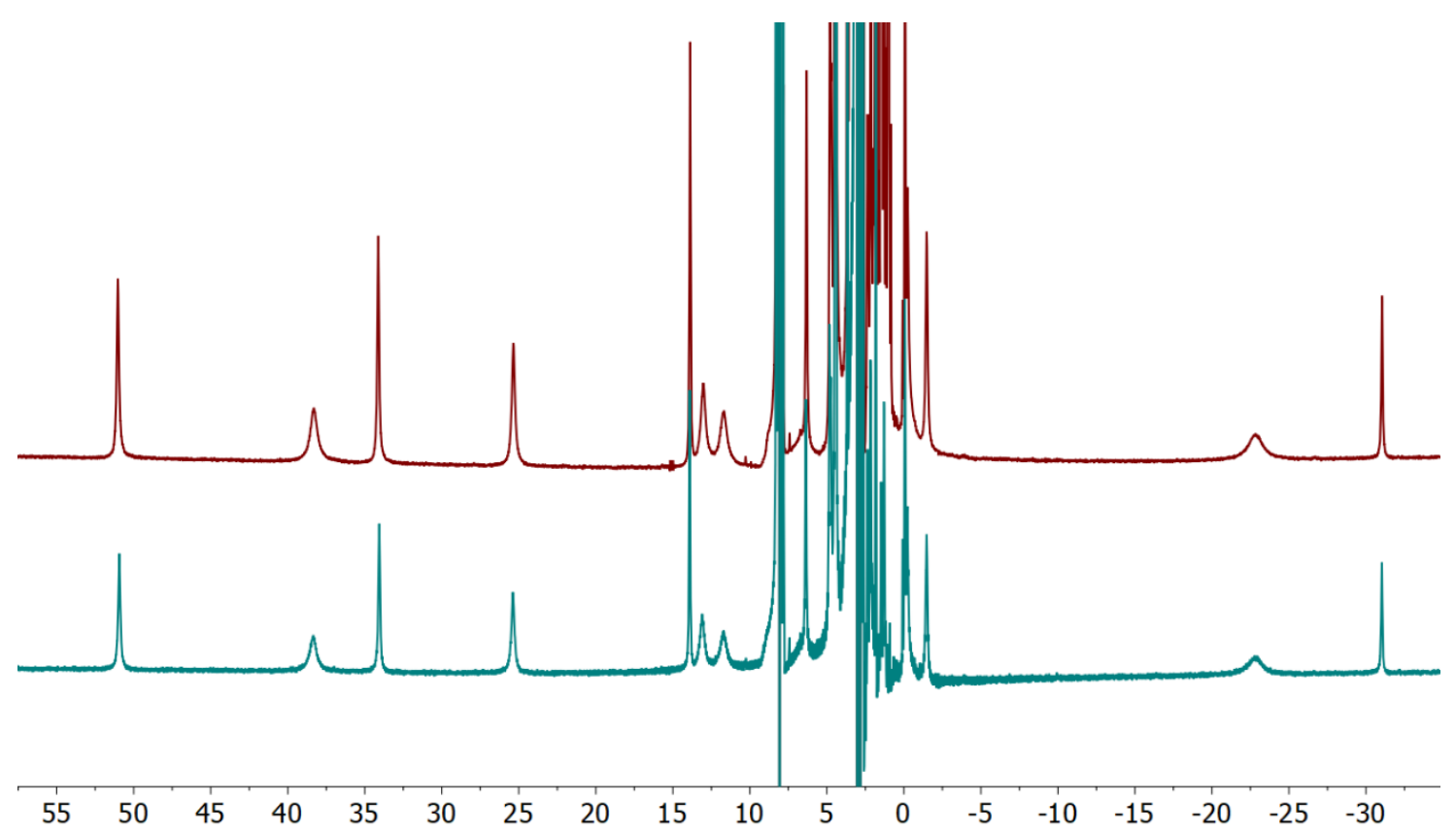

Figure S30 ${ }^{1} \mathrm{H}$ NMR spectra $\left(\mathrm{DMF}, 25{ }^{\circ} \mathrm{C}\right)$ of $\left({ }^{\mathrm{BBN} P D P}{ }^{\mathrm{tBu}}\right) \mathrm{Fe}\left(\mathrm{NH}_{2}\right)_{2}$ illustrating the stability of this complex toward free bromide. Bottom: spectrum of $\left({ }^{\mathrm{BBN} P D P}{ }^{\mathrm{BB} u}\right) \mathrm{Fe}\left(\mathrm{NH}_{2}\right)_{2}$ prior to addition of $\left[\mathrm{Bu}_{4} \mathrm{~N}\right][\mathrm{Br}]$. Top: spectrum of ( $\left.{ }^{\mathrm{BBN} P D P}{ }^{\mathrm{BBu}}\right) \mathrm{Fe}\left(\mathrm{NH}_{2}\right)_{2}$ after addition of 10 equivalents of $\left[\mathrm{Bu}_{4} \mathrm{~N}\right][\mathrm{Br}]$. X-axis units in ppm.

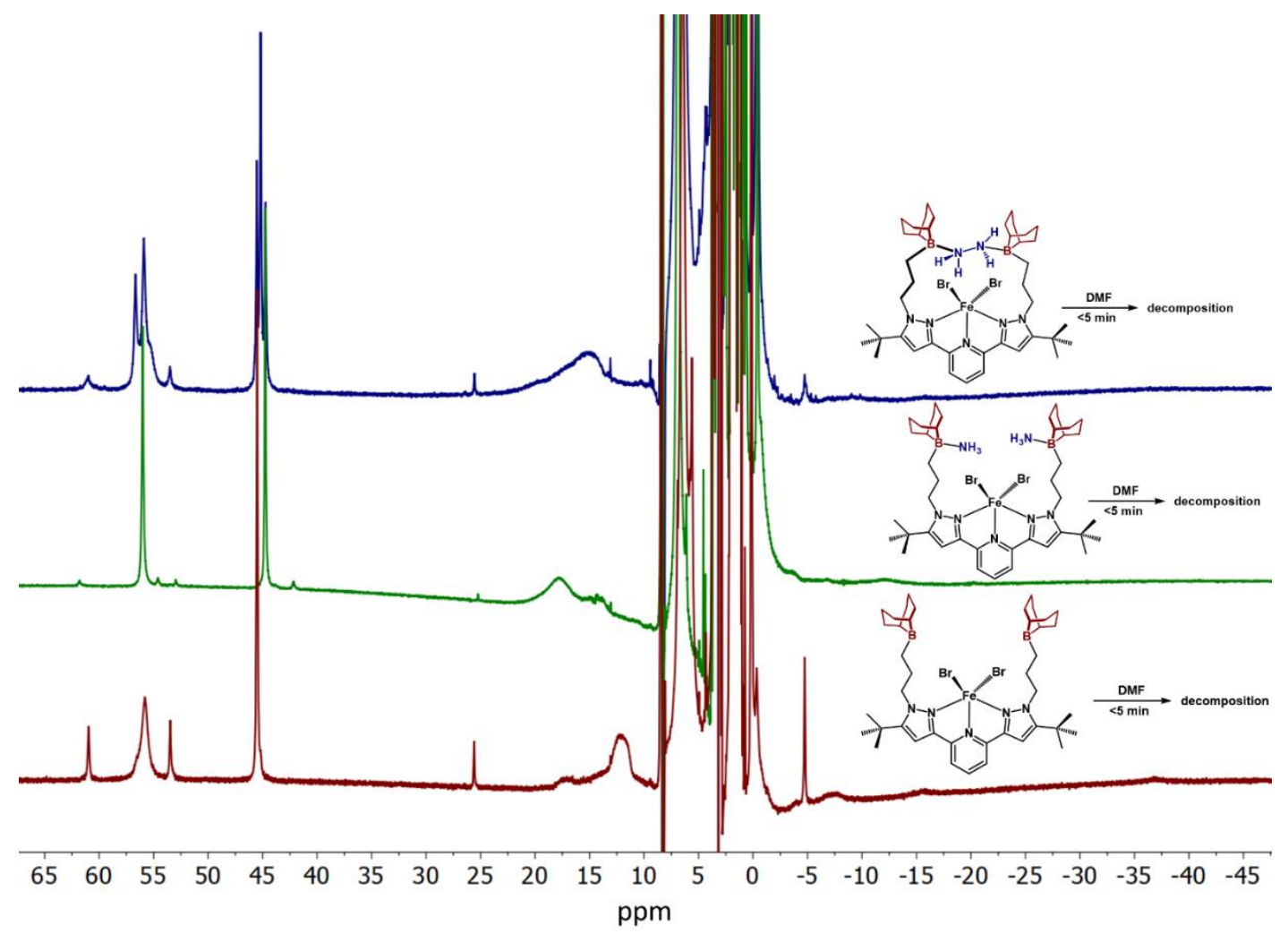

Figure S31 ${ }^{1} \mathrm{H}$ NMR spectra (DMF, $25^{\circ} \mathrm{C}$ ) highlighting the instability of ( $\left.{ }^{\mathrm{BBN}} \mathrm{PDP}^{\mathrm{tBu}}\right) \mathrm{FeBr}_{2}$ (bottom), ( $\left.{ }^{\mathrm{BBN} P D P}{ }^{\mathrm{tBu}}\right) \mathrm{FeBr}_{2}\left(\mathrm{NH}_{3}\right)_{2}$ (middle), and ( $\left.{ }^{\mathrm{BBN} P D P}{ }^{\mathrm{BBu}}\right) \mathrm{FeBr}_{2}\left(\mathrm{~N}_{2} \mathrm{H}_{4}\right)$ (top) in DMF solvent. In each case, a sample (approx. $2 \mathrm{mM}, \mathrm{DMF}$ ) was prepared in a glovebox and an ${ }^{1} \mathrm{H}$ NMR spectrum was acquired within 5 min. 


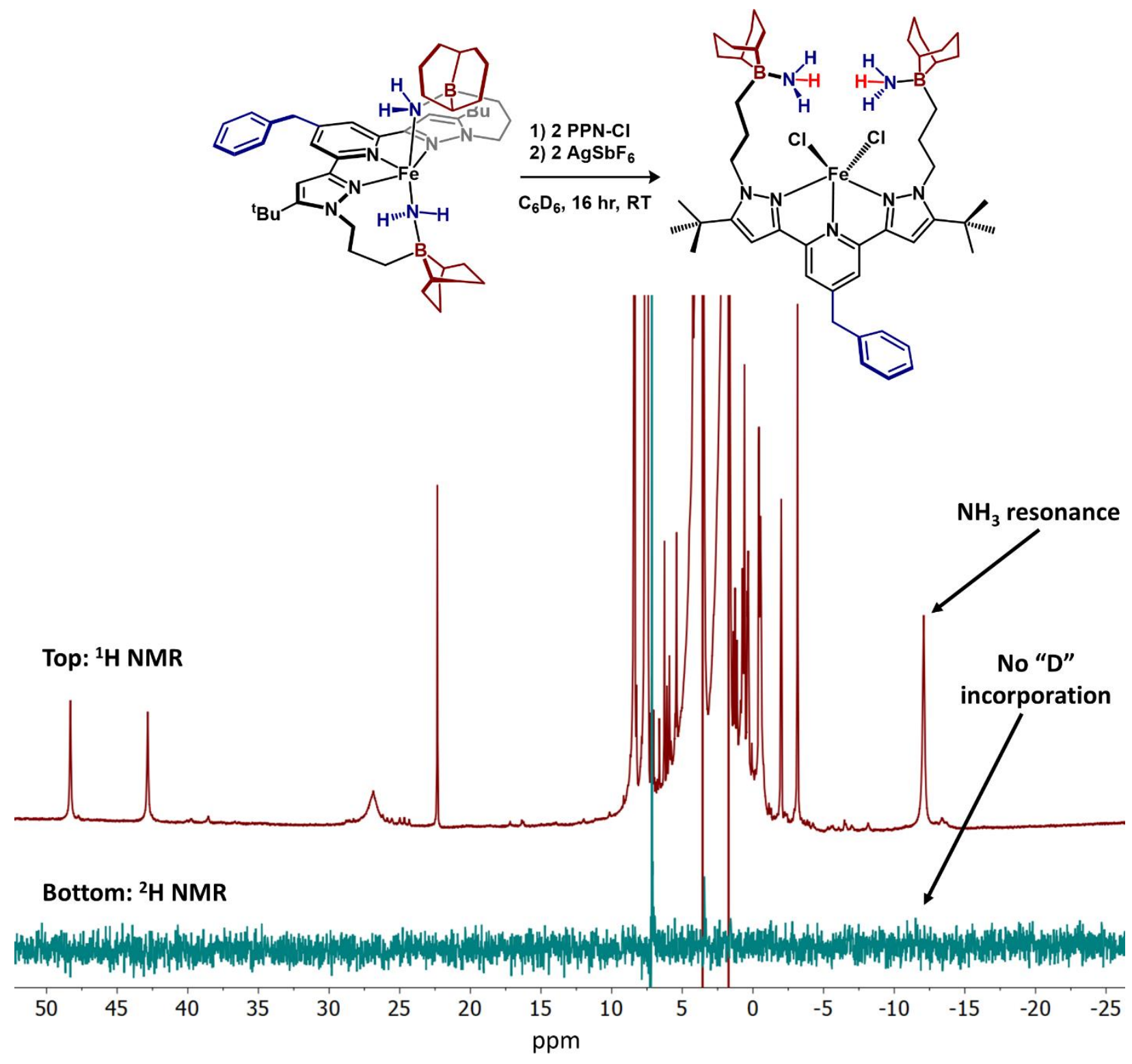

Figure S32 Crude ${ }^{1} \mathrm{H}$ NMR (top) and ${ }^{2} \mathrm{H}$ NMR (bottom) spectra of oxidation of (benzyl- ${ }^{\mathrm{BBN}} \mathrm{PDP}^{\mathrm{tBu}}$ ) Fe $\left(\mathrm{NH}_{2}\right)_{2}$ with $\mathrm{AgSbF}_{6}$ in the presence of chloride. Reactions were performed in $\mathrm{C}_{6} \mathrm{D}_{6}$ and the spectra are recorded in THF. 


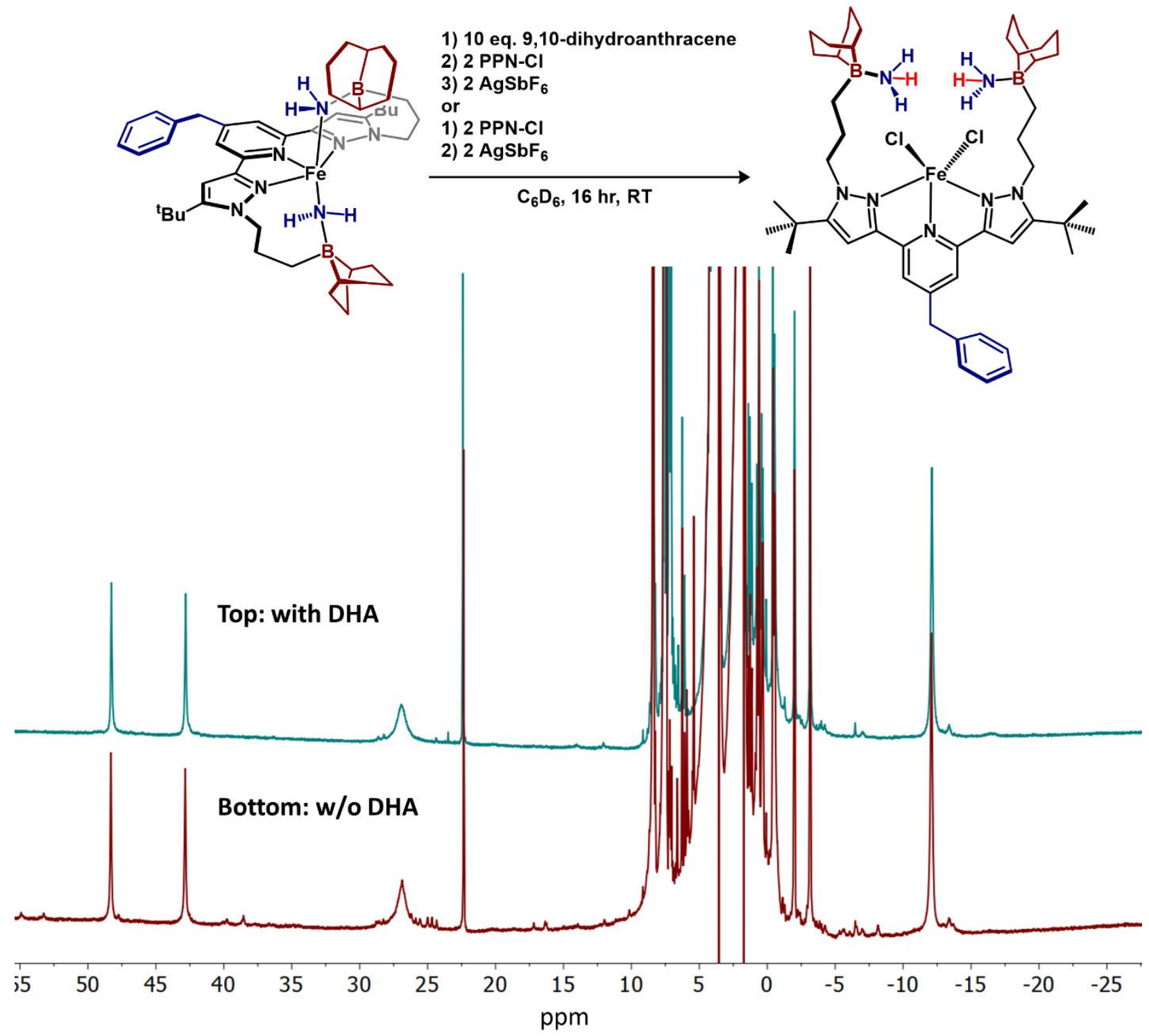

Figure S33 Crude ${ }^{1} \mathrm{H}$ NMR spectra of the role of a weak $\mathrm{H}$-atom donor, 9,10-dihydroanthracene, on the oxidation of (benzyl- $\left.{ }^{\mathrm{BBN}} \mathrm{PDP}{ }^{\mathrm{tBu}}\right) \mathrm{Fe}\left(\mathrm{NH}_{2}\right)_{2}$ with $\mathrm{AgSbF}_{6}$ in the presence of chloride. Reactions were performed in $\mathrm{C}_{6} \mathrm{D}_{6}$ and the spectra are recorded in THF. The top spectrum corresponds to the reaction in the presence of 10 equiv. DHA, the reaction corresponding to bottom spectrum did not contain DHA. 

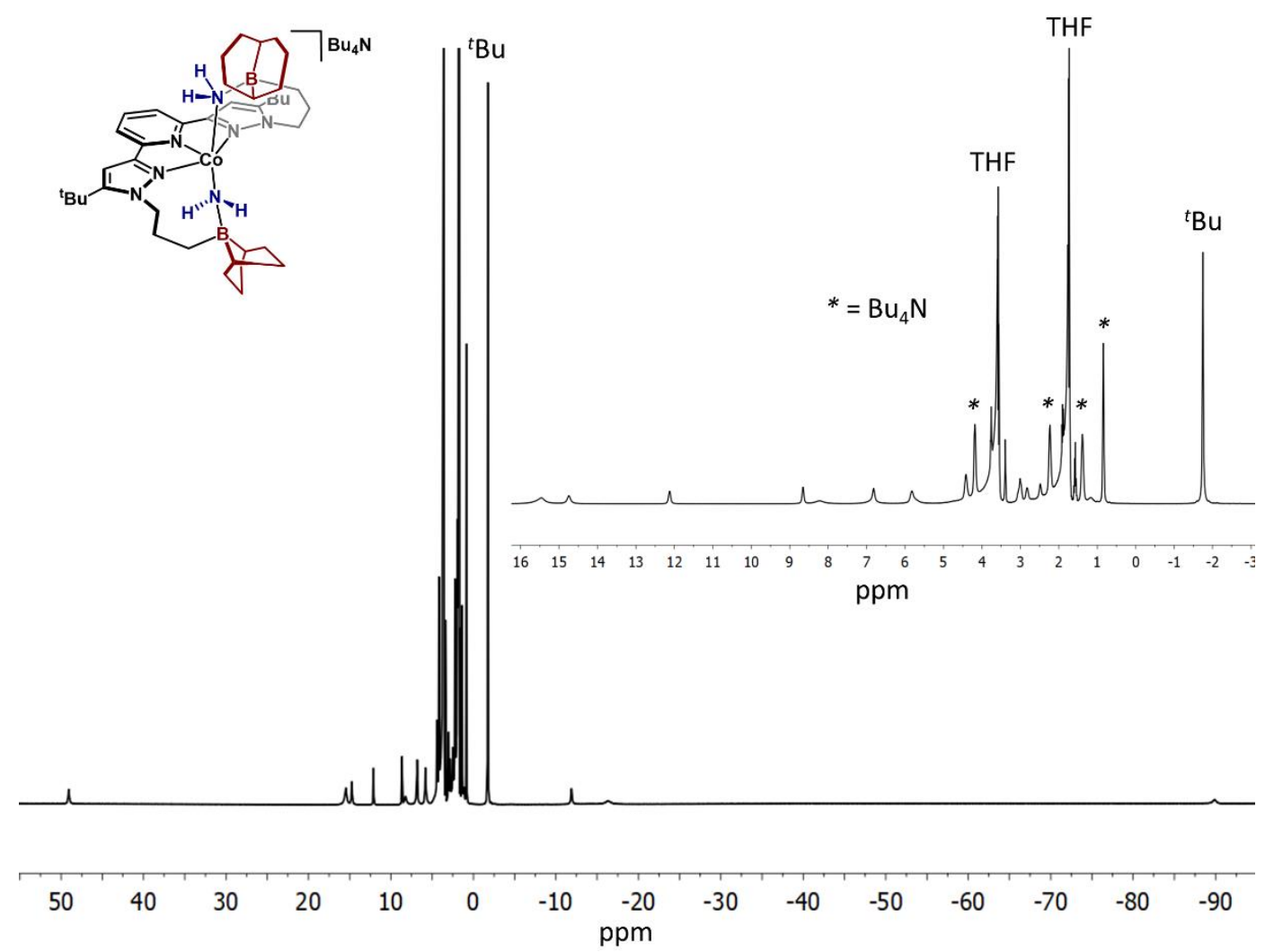

Figure S34 ${ }^{1} \mathrm{H}$ NMR spectrum $\left(\mathrm{THF}, 25^{\circ} \mathrm{C}\right)$ of $\left[\mathrm{Bu}_{4} \mathrm{~N}\right]\left[\left({ }^{\mathrm{BBN}} \mathrm{PDP}{ }^{\mathrm{tBu}}\right) \mathrm{Co}\left(\mathrm{NH}_{2}\right)_{2}\right]$.

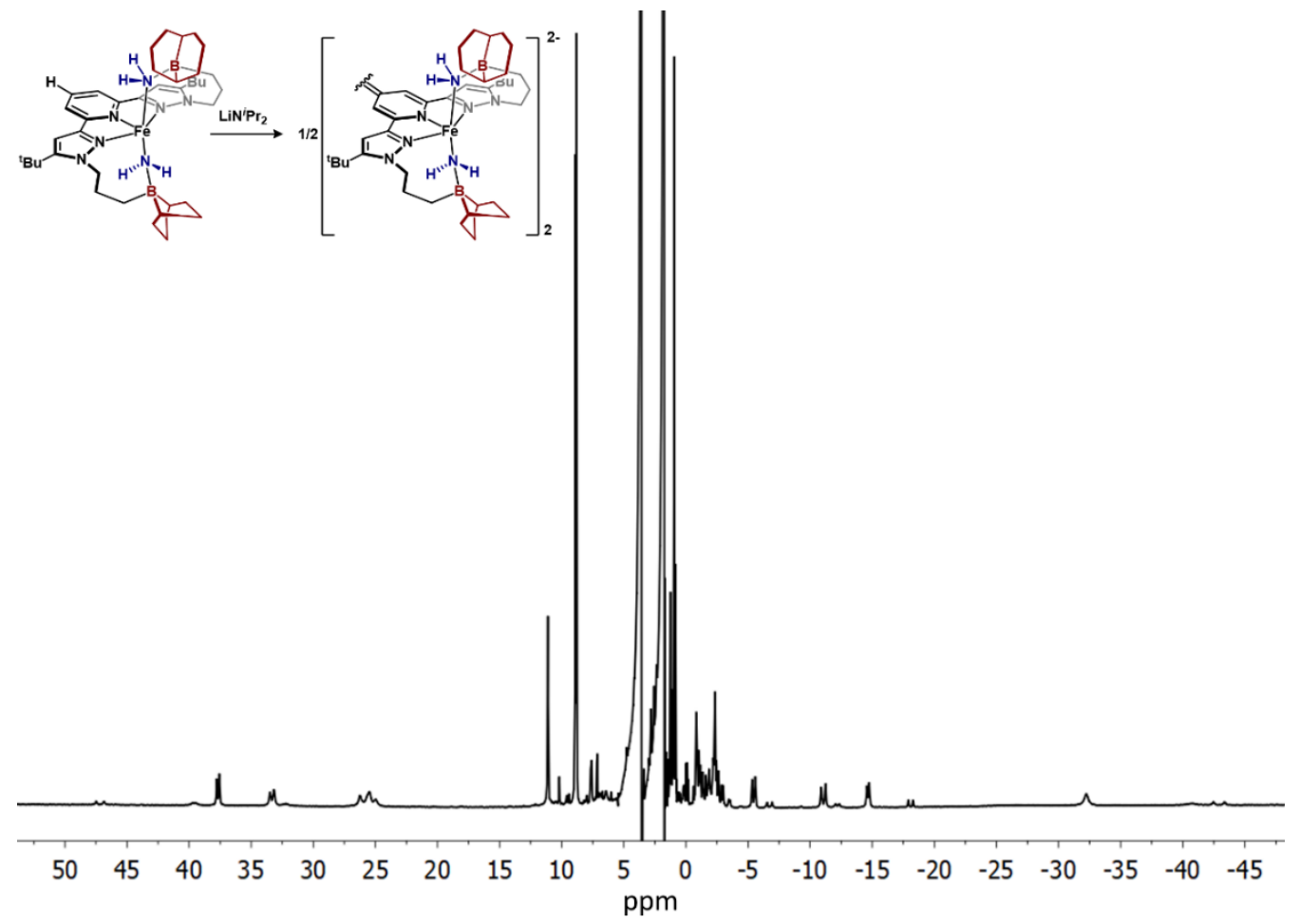

Figure S35 ${ }^{1} \mathrm{H}$ NMR spectrum (THF, ambient temperature) of crude reaction between ( $\left.{ }^{\mathrm{BBN} P D P}{ }^{\mathrm{BBu}}\right) \mathrm{Fe}\left(\mathrm{NH}_{2}\right)_{2}$ and lithium diisopropylamide. 


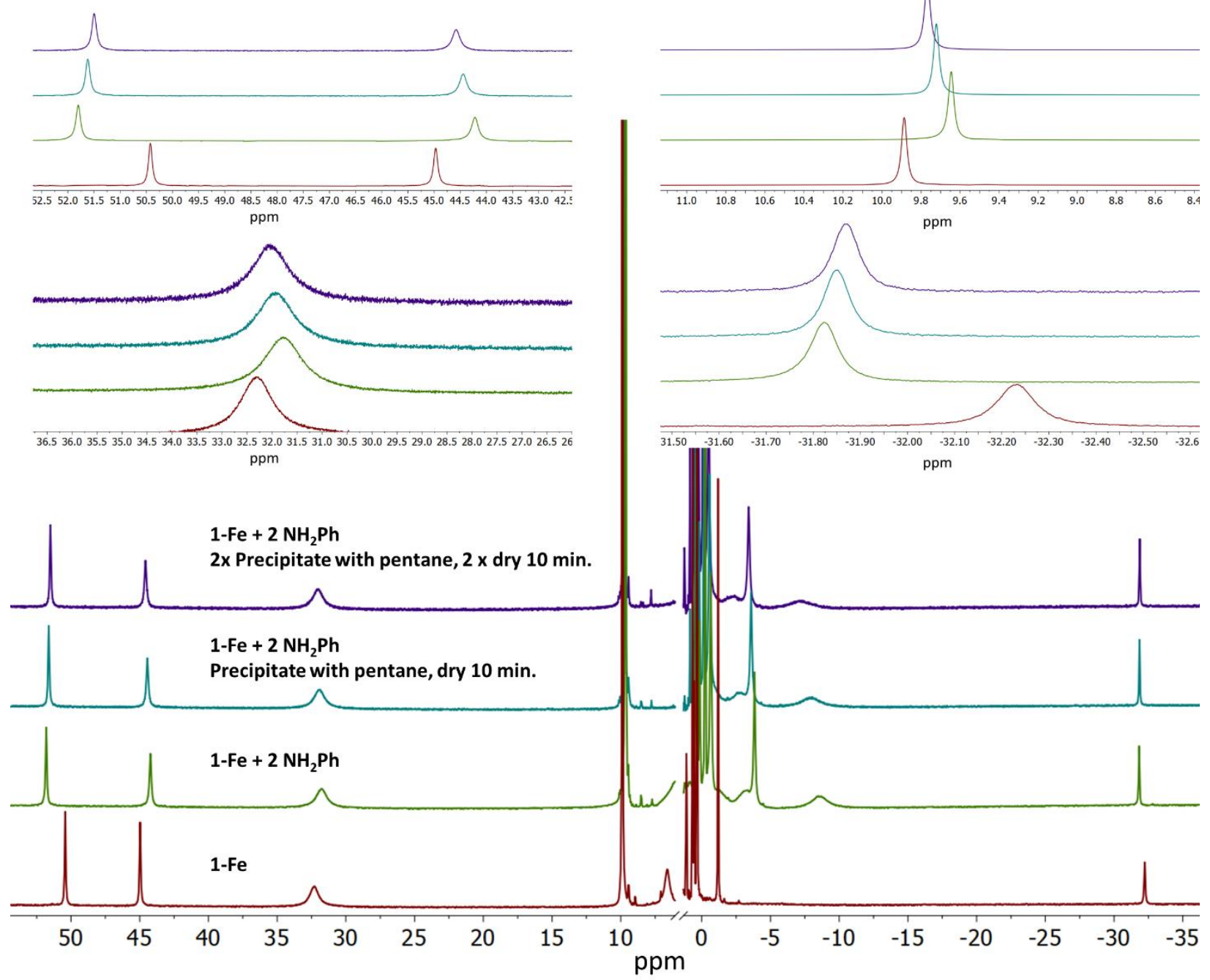

Figure S36 Overlay of ${ }^{1} \mathrm{H}$ NMR spectra recorded in THF at $25{ }^{\circ} \mathrm{C}$. Bottom (red): ( $\left.{ }^{\mathrm{BBN}} \mathrm{PDP}{ }^{\mathrm{tBu}}\right) \mathrm{FeBr}_{2}$. Middle (green): $\left({ }^{\mathrm{BBN} P D P}{ }^{\mathrm{BBu}}\right) \mathrm{FeBr}_{2}\left(\mathrm{NH}_{2} \mathrm{Ph}\right)_{2}$ generated from the addition of two equivalents $\mathrm{NH}_{2} \mathrm{Ph}$ to $\left.{ }^{\left(B B N P D P^{t B u}\right.}\right) \mathrm{FeBr}_{2}$. Middle (blue): after precipitation of the product and drying in vacuo for ten minutes. Top (purple): after a $2^{\text {nd }}$ iteration of precipitation and drying. 


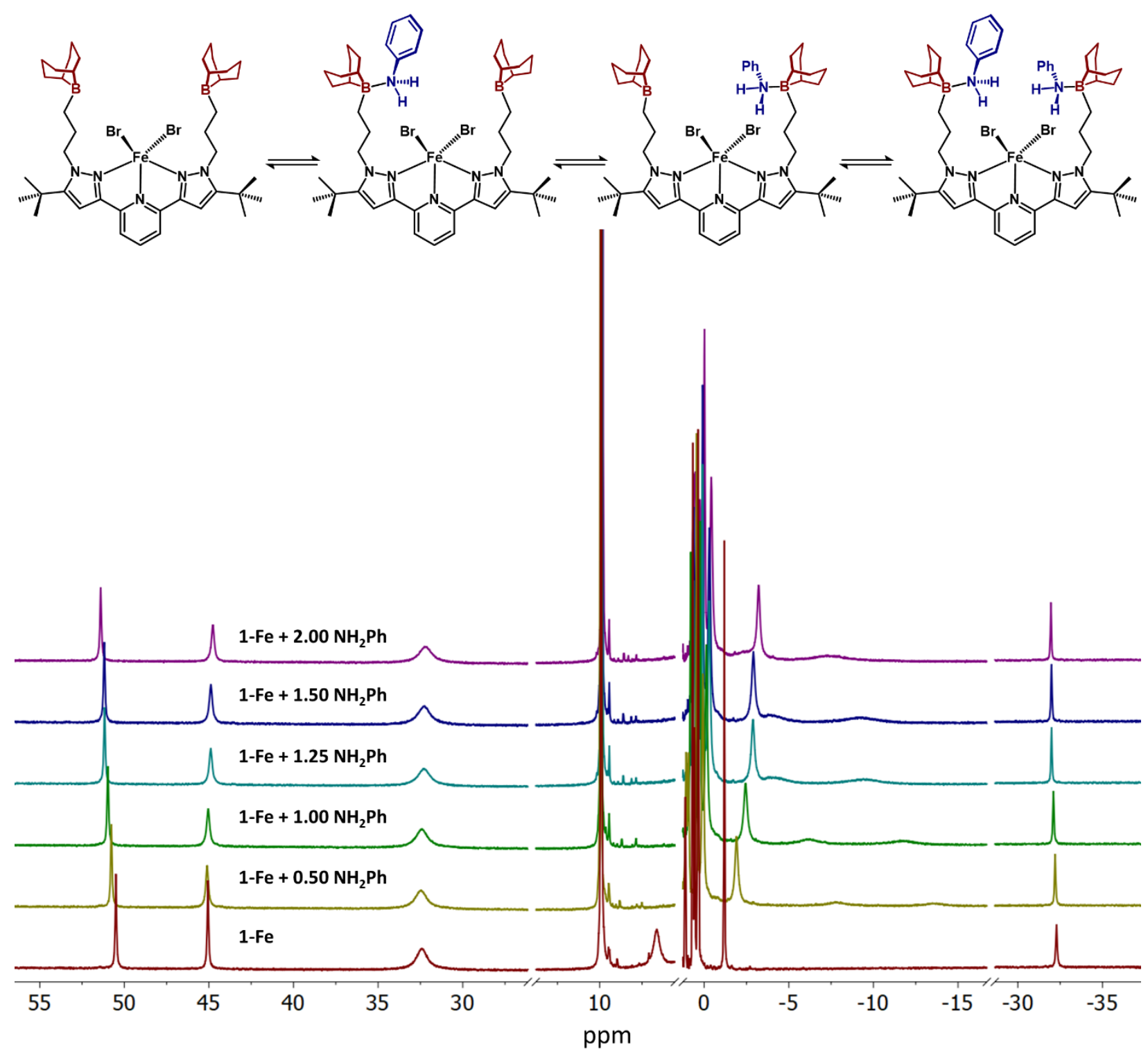

Figure S37 Overlay of ${ }^{1} \mathrm{H}$ NMR spectra of titration experiment recorded in THF at $25{ }^{\circ} \mathrm{C}$. Bottom (red): ( $\left.{ }^{\mathrm{BBN}} \mathrm{PDP}{ }^{\mathrm{BBu}}\right) \mathrm{FeBr}_{2}$. Each subsequent spectrum displays increasing amounts of $\mathrm{NH}_{2} \mathrm{Ph}$ (up to 2 equiv.; labeled in figure) highlighting the reversibility of B-N bond formation. 


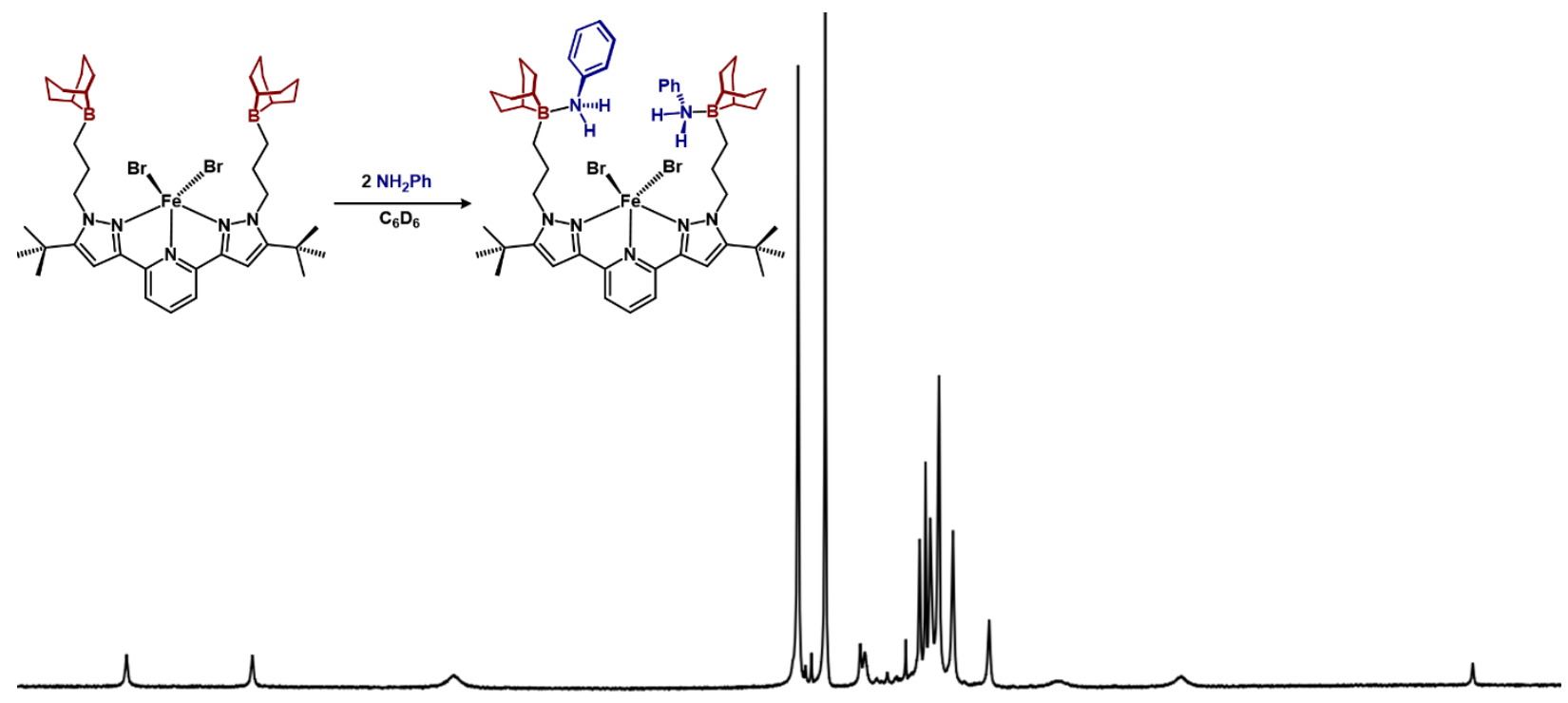

$\begin{array}{lllllllllllllllllll}55 & 50 & 45 & 40 & 35 & 30 & 25 & 20 & 15 & 10 & 5 & 0 & -5 & -10 & -15 & -20 & -25 & -30 & -35\end{array}$

Figure S38 ${ }^{1} \mathrm{H}$ NMR spectrum $\left(\mathrm{C}_{6} \mathrm{D}_{6}, 25^{\circ} \mathrm{C}\right)$ of $\left({ }^{\mathrm{BBN} P D P}{ }^{\mathrm{BBu}}\right) \mathrm{FeBr}_{2}\left(\mathrm{NH}_{2} \mathrm{Ph}\right)_{2}$.

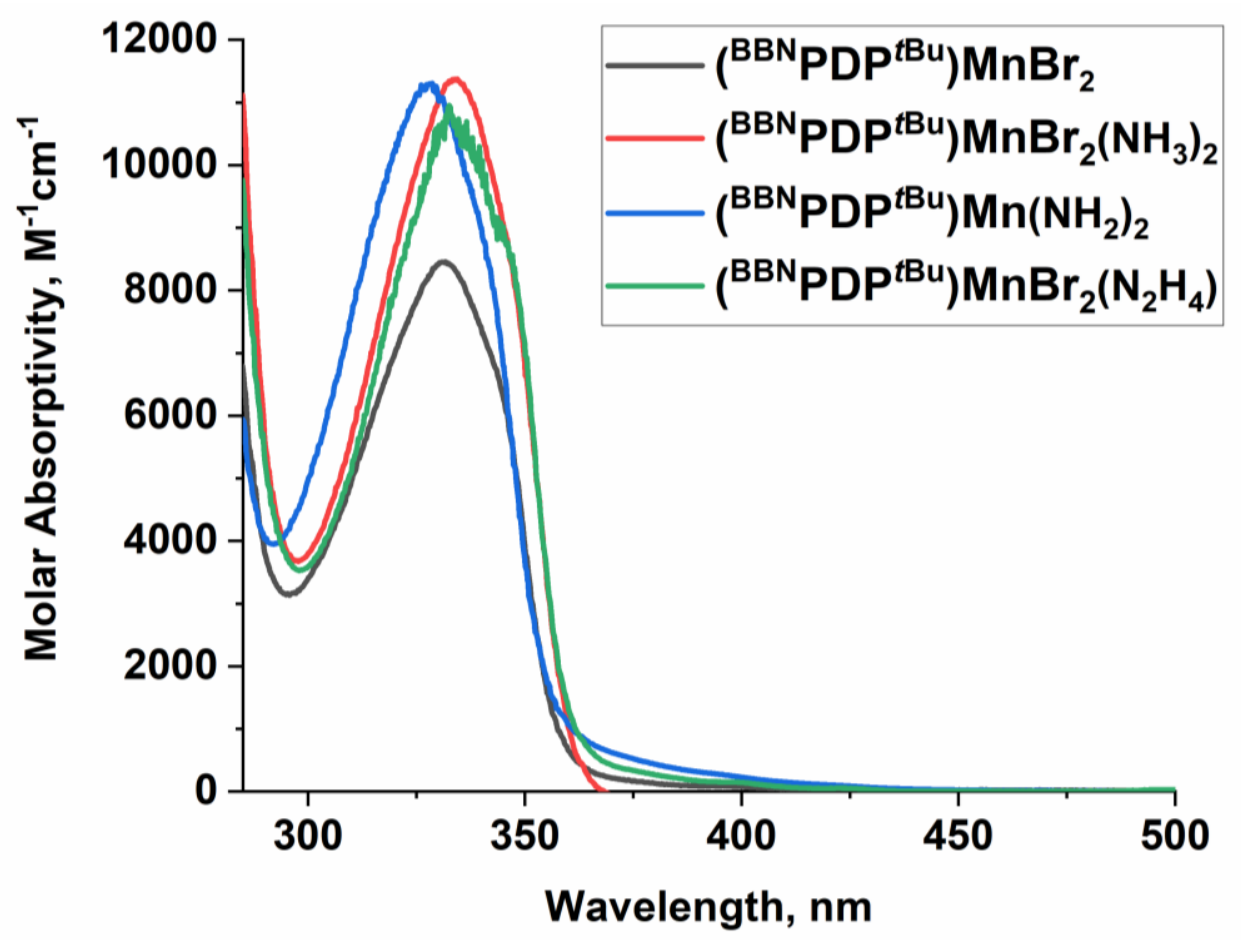

Figure S39 Electronic absorption spectra (THF, ambient temperature) of ( $\left.{ }^{\mathrm{BBN}} \mathrm{PDP}^{\mathrm{tBu}}\right) \mathrm{MnBr}_{2}$ (black),

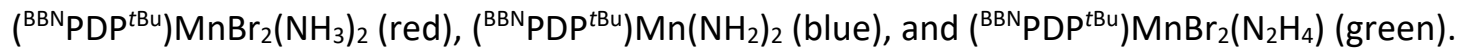




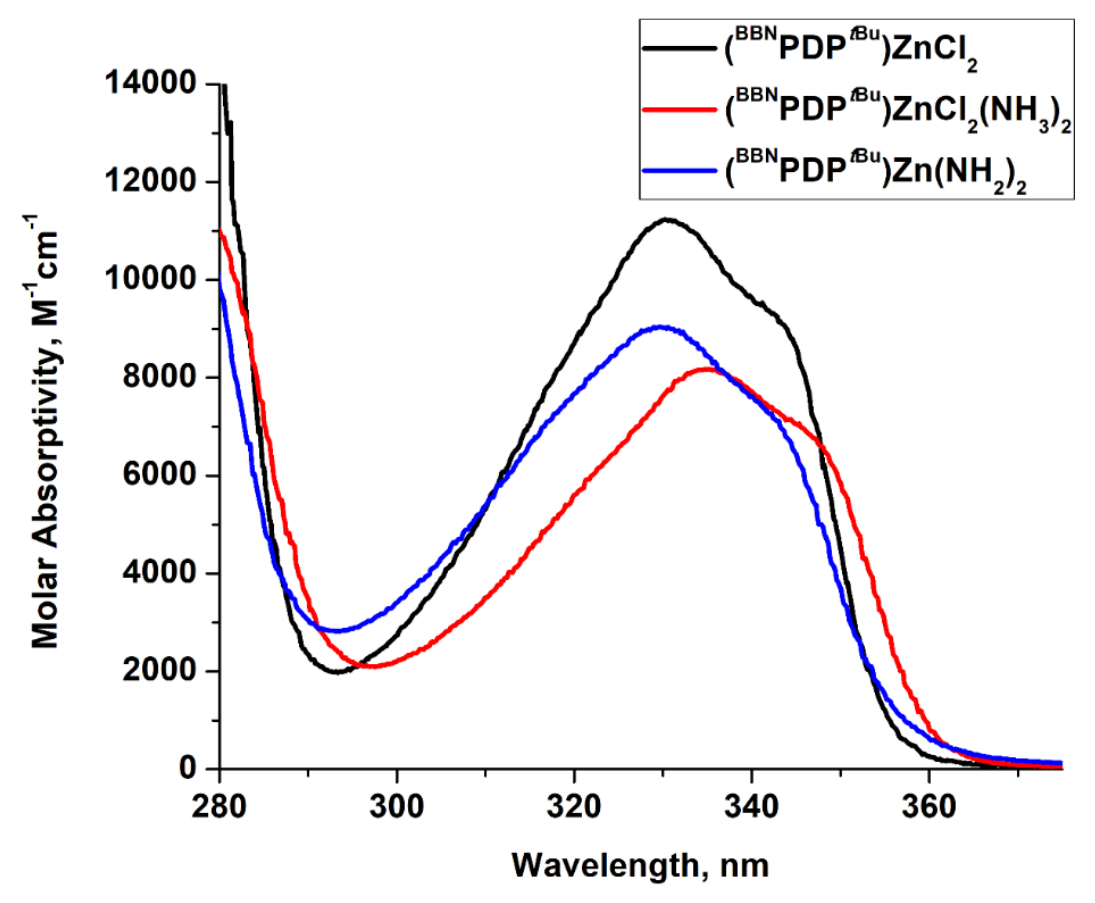

Figure $\mathbf{S 4 0}$ Electronic absorption spectra (THF, ambient temperature) of ( $\left.{ }^{\mathrm{BBN}}{ }^{\mathrm{PDP}}{ }^{\mathrm{tBu}}\right) \mathrm{ZnCl}_{2}$ (black),

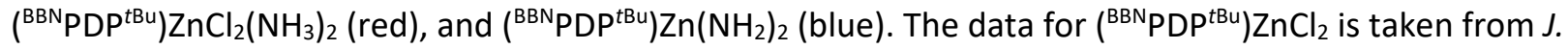
Am. Chem. Soc. 2017, 139, 18194-18197 for comparison.

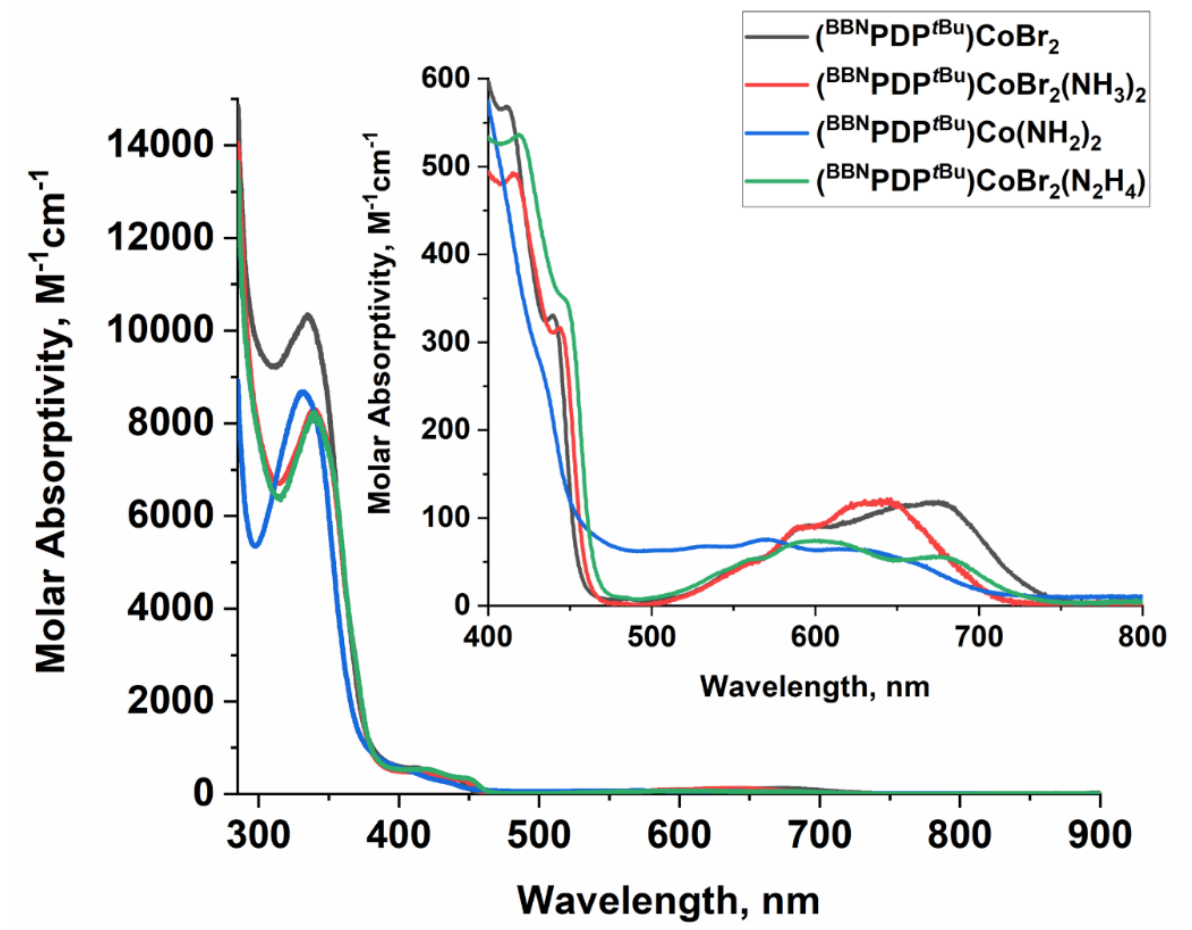

Figure S41 Electronic absorption spectra (THF, ambient temperature) of ( $\left.{ }^{\mathrm{BBN}} \mathrm{PDP}^{\mathrm{tBu}}\right) \mathrm{CoBr}_{2}$ (black), $\left({ }^{B B N P D P}{ }^{t B u}\right) \mathrm{CoBr}_{2}\left(\mathrm{NH}_{3}\right)_{2}$ (red), ( $\left.{ }^{\mathrm{BBN} P D P}{ }^{\mathrm{BBu}}\right) \mathrm{Co}\left(\mathrm{NH}_{2}\right)_{2}$ (blue), and $\left({ }^{\mathrm{BBN} P D P}{ }^{t B u}\right) \mathrm{CoBr}_{2}\left(\mathrm{~N}_{2} \mathrm{H}_{4}\right)$ (green). The inset is an expansion of the lower energy region. 


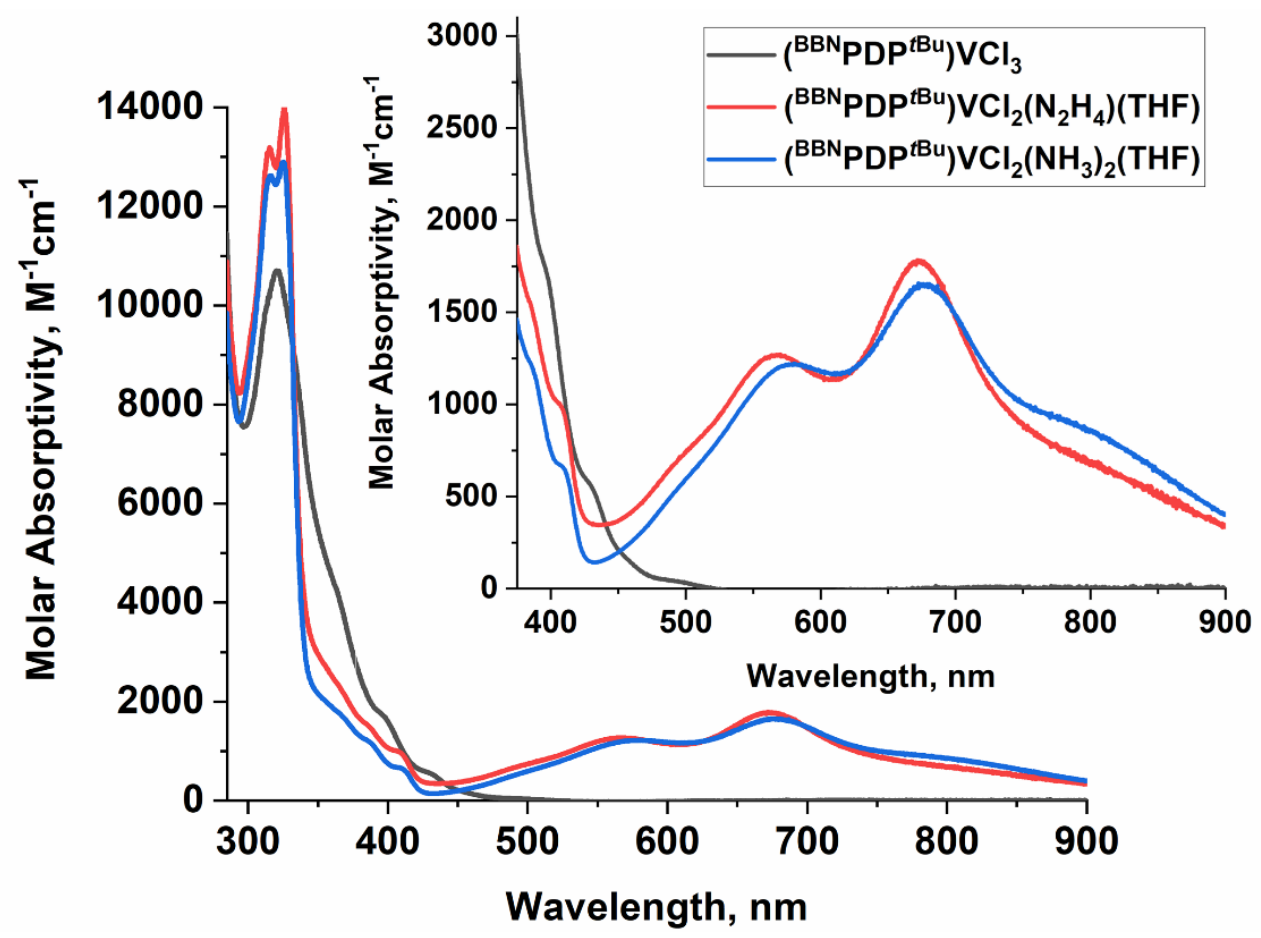

Figure S42 Electronic absorption spectra (THF, ambient temperature) of ( $\left.{ }^{\mathrm{BBN}} \mathrm{PDP}^{\mathrm{tBu}}\right) \mathrm{VCl}_{3}$ (black), $\left({ }^{\text {BBN PDP }}{ }^{\mathrm{tBu}}\right) \mathrm{VCl}_{2}\left(\mathrm{NH}_{3}\right)_{2}$ (THF) (blue), and ( $\left.{ }^{\mathrm{BBN} P D P}{ }^{\mathrm{tBu}}\right) \mathrm{VCl}_{2}\left(\mathrm{~N}_{2} \mathrm{H}_{4}\right)$ (THF) (red). The inset is an expansion of the lower energy region.

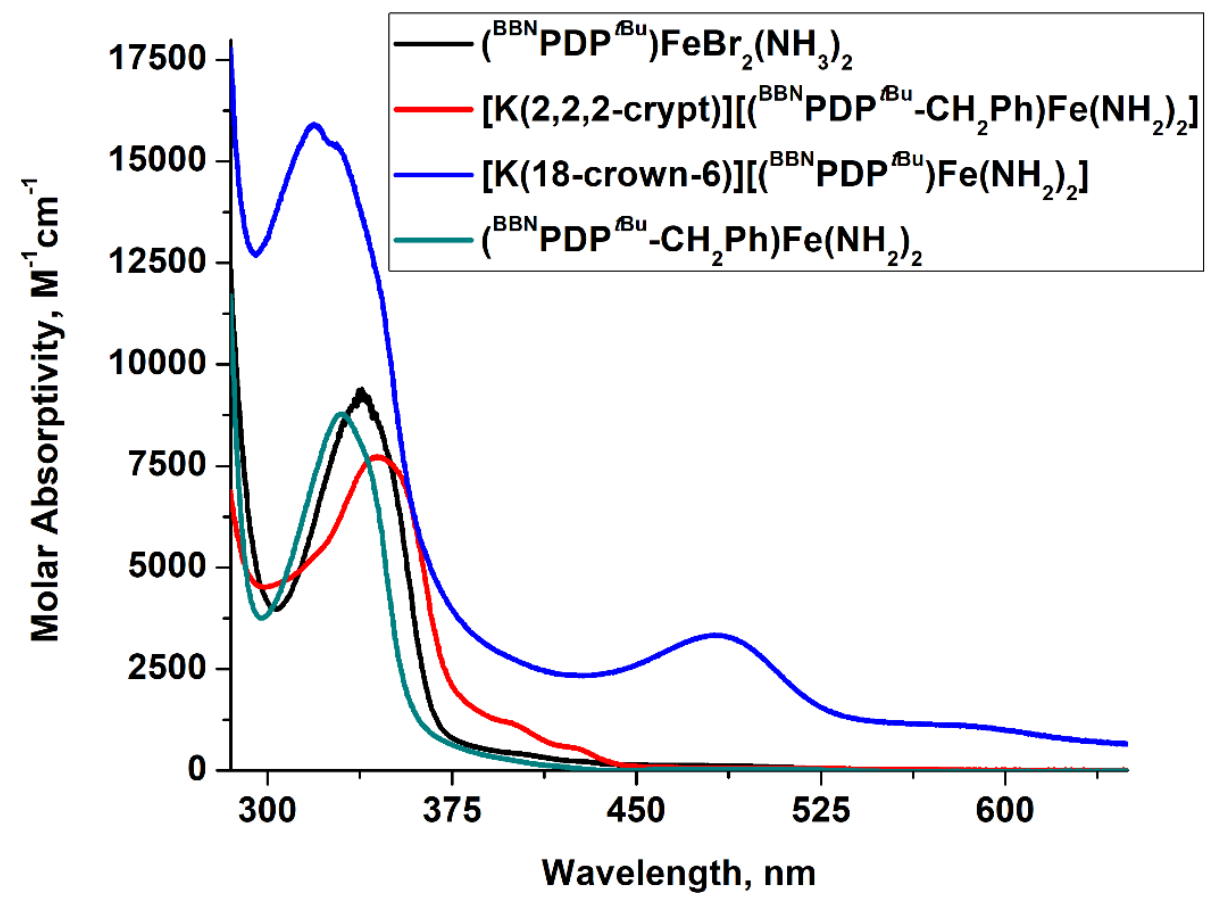

Figure S43 Electronic absorption spectra (THF, ambient temperature) of ( $\left.{ }^{\mathrm{BBN} P D P}{ }^{\mathrm{tBu}}\right) \mathrm{FeBr}_{2}\left(\mathrm{NH}_{3}\right)_{2}$ (black), $[\mathrm{K}(2,2,2-\mathrm{crypt})]\left[\left(^{\mathrm{BBN} P D P^{t B u}}-\mathrm{CH}_{2} \mathrm{Ph}\right) \mathrm{Fe}\left(\mathrm{NH}_{2}\right)_{2}\right]$ (red), [K(18-crown-6)][( $\left.\left.{ }^{\mathrm{BBN} P D P^{t B u}}\right) \mathrm{Fe}\left(\mathrm{NH}_{2}\right)_{2}\right]$ (blue), and ( $\left.{ }^{\mathrm{BBN}} \mathrm{PDP} \mathrm{P}^{\mathrm{tBu}}-\mathrm{CH}_{2} \mathrm{Ph}\right) \mathrm{Fe}\left(\mathrm{NH}_{2}\right)_{2}$ (teal). 


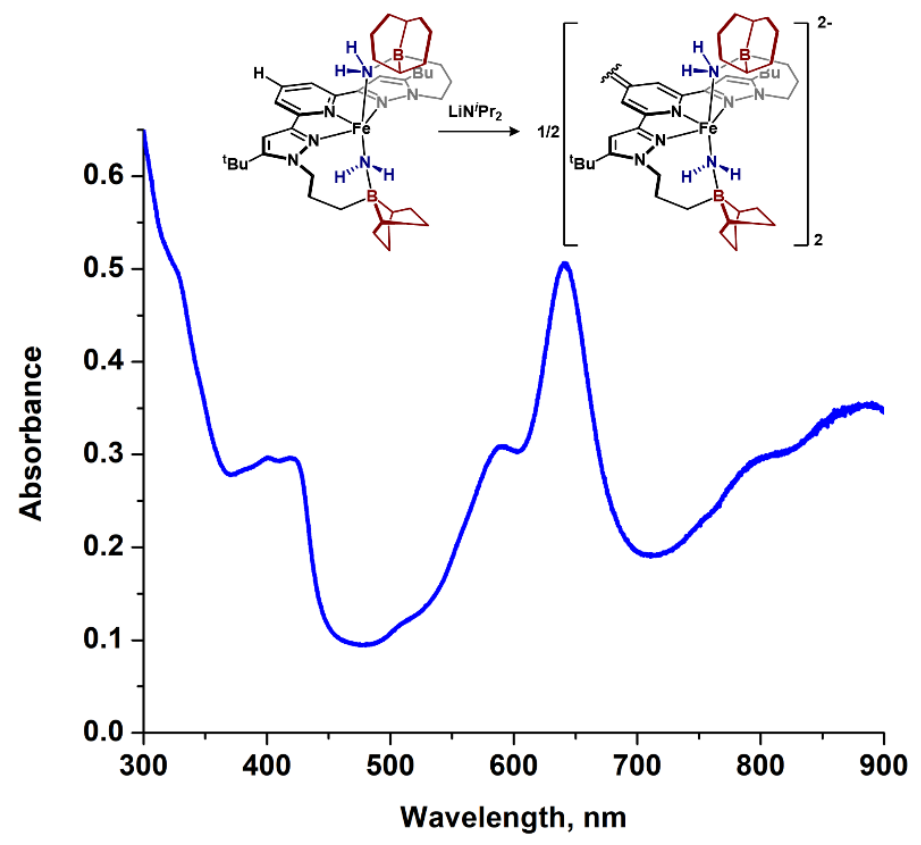

Figure S44 Electronic absorption spectra (THF, ambient temperature) of reaction between $\left({ }^{B B N} P P^{t B u}\right) F e\left(\mathrm{NH}_{2}\right)_{2}$ and lithium diisopropylamide. 

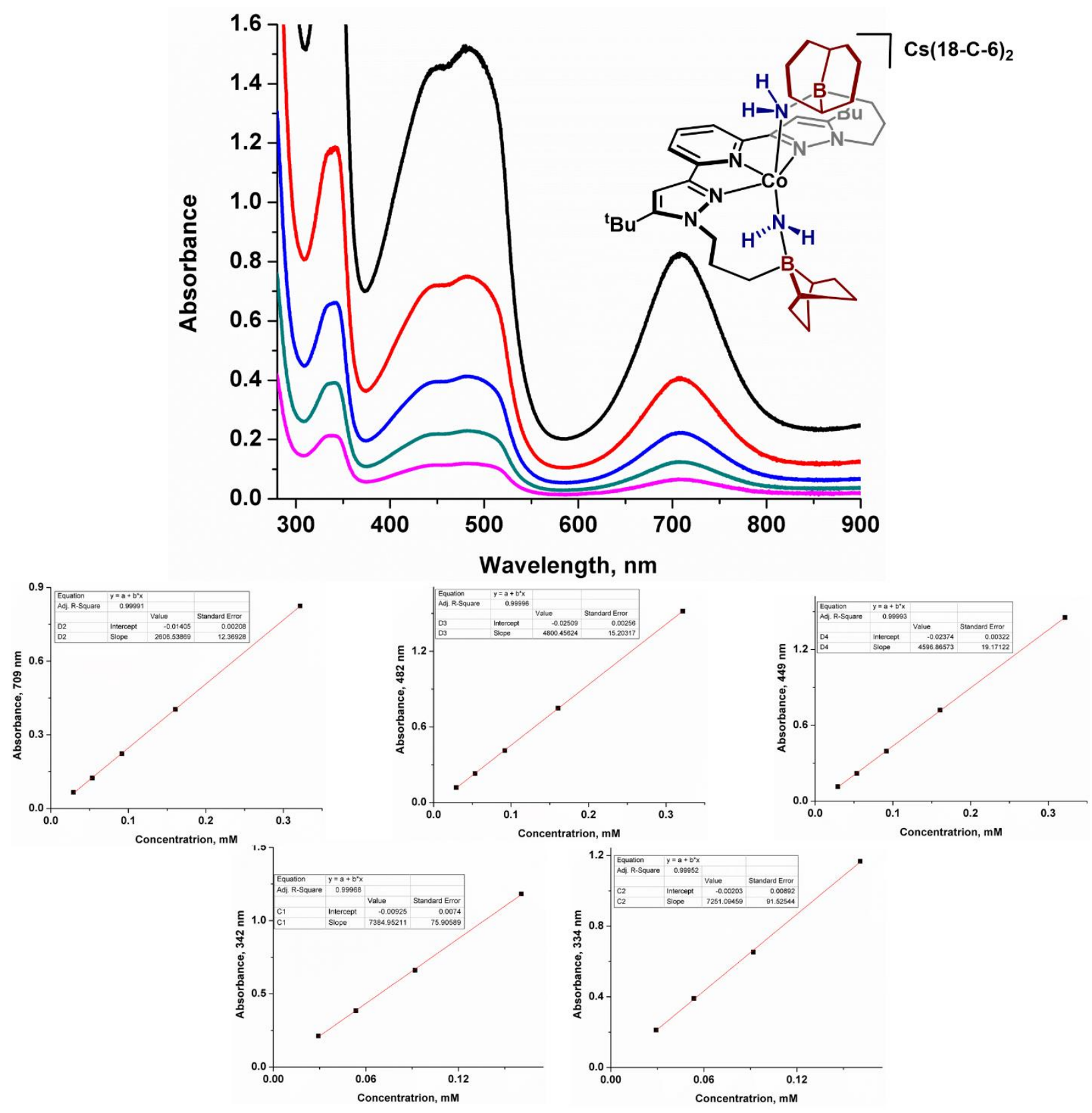

Figure S45 Beer's Law analysis of $\left[\mathrm{Cs}(18-\mathrm{crown}-6)_{2}\right]\left[\left({ }^{\mathrm{BBN}} \mathrm{PDP}{ }^{\mathrm{tBu}}\right) \mathrm{Co}\left(\mathrm{NH}_{2}\right)_{2}\right]$ in THF at ambient temperature. 

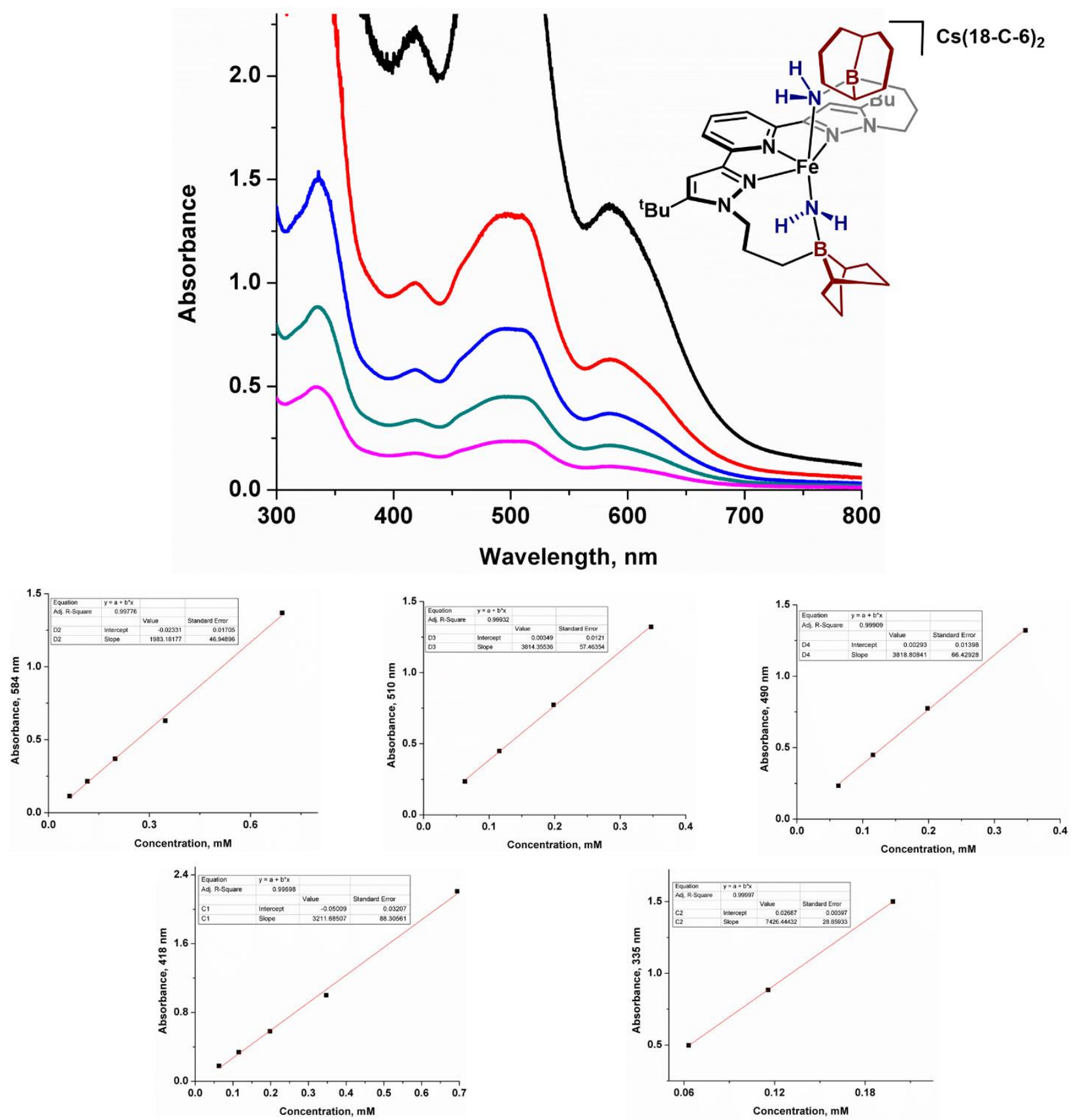

Figure S46 Beer's Law analysis of [Cs(18-crown-6 $\left.)_{2}\right]\left[\left({ }^{\mathrm{BBN} P D P}{ }^{\mathrm{tBu}}\right) \mathrm{Fe}\left(\mathrm{NH}_{2}\right)_{2}\right]$ in THF at ambient temperature. 

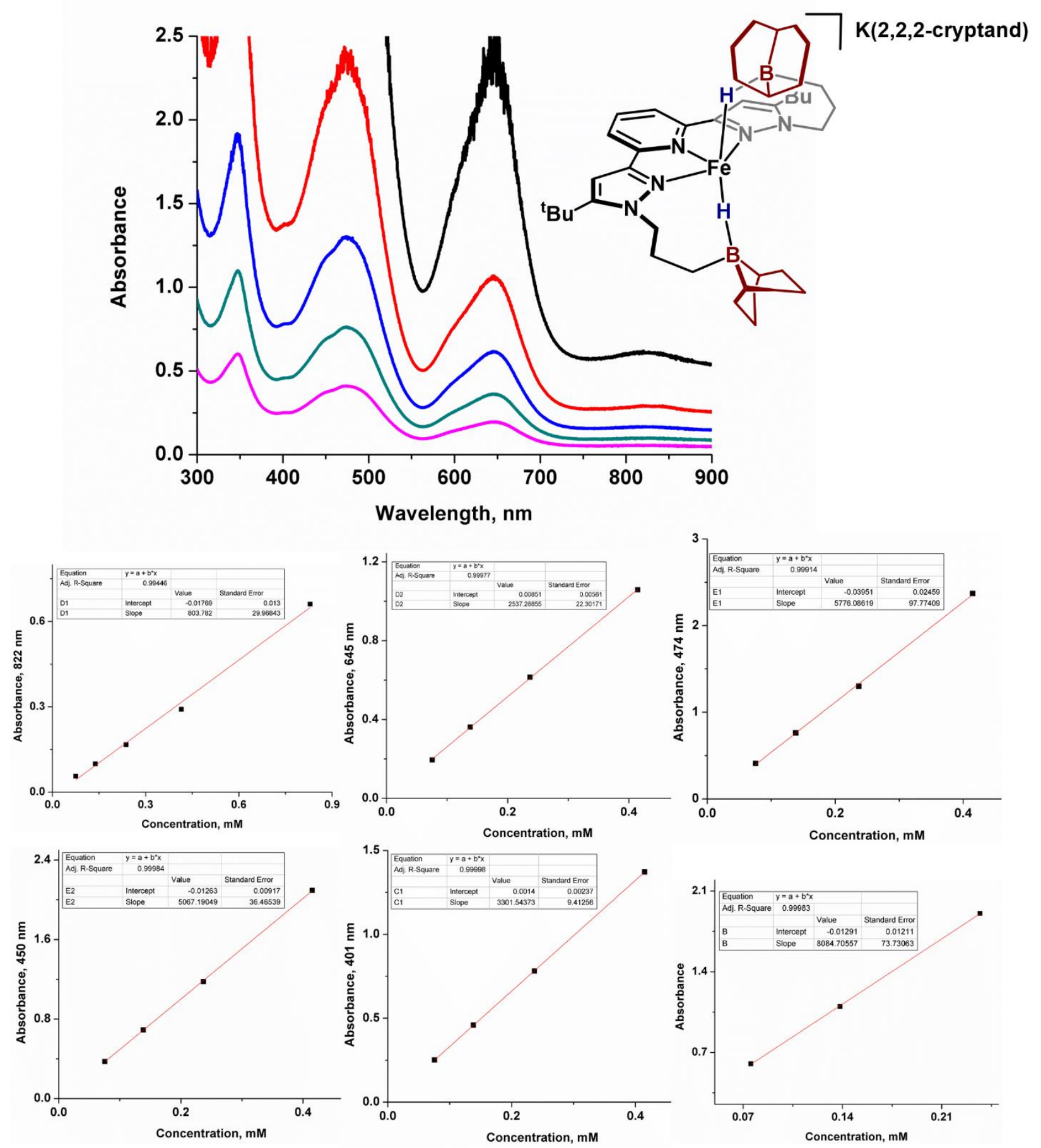

Figure S47 Beer's Law analysis of [K(2,2,2-cryptand) $]\left[\left({ }^{(B B N P D P}{ }^{t B u}\right) F e(H)_{2}\right]$ in THF at ambient temperature. 

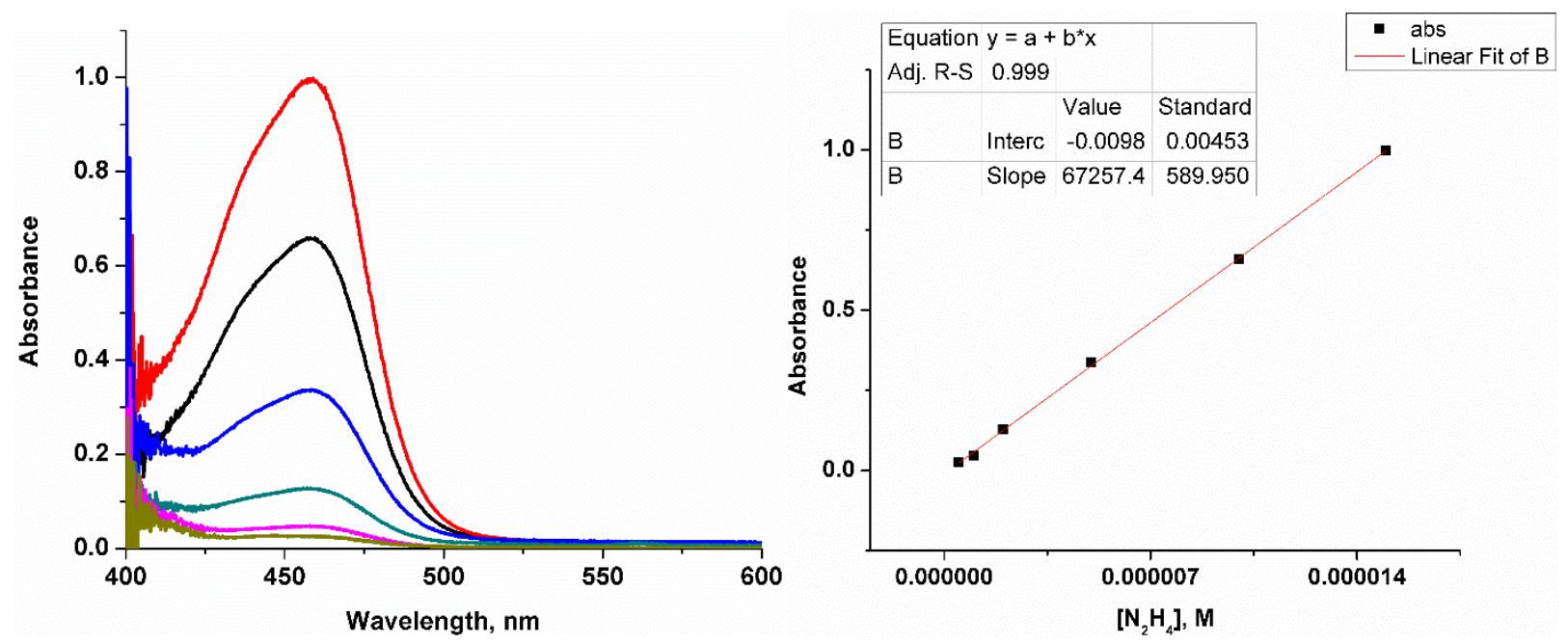

Figure S48 Calibration curve for hydrazine detection. Left: UV Vis traces. Right: Linear fit of $\left[\mathrm{N}_{2} \mathrm{H}_{4}\right]$ vs. absorbance.

Table S1. Amount of $\mathrm{N}_{2} \mathrm{H}_{4}$ detected for each set of conditions.

\begin{tabular}{|c|c|c|c|c|}
\hline Entry & Metal & Oxidant & Solvent & $\begin{array}{l}\text { Equivalents } \\
\mathrm{N}_{2} \mathrm{H}_{4} \text { detected }\end{array}$ \\
\hline 1 & $\mathrm{Fe}$ & $\mathrm{AgSbF}_{6}$ & THF & 0 \\
\hline 2 & $\mathrm{Fe}$ & $\mathrm{AgSbF}_{6}$ & DMF & 0 \\
\hline 3 & $\mathrm{Fe}$ & {$\left[\mathrm{Cp}_{2} \mathrm{Fe}\right]\left[\mathrm{BPh} \mathrm{h}_{4}\right]$} & THF & 0 \\
\hline 4 & $\mathrm{Fe}$ & {$\left[\mathrm{Cp}_{2} \mathrm{Fe}\right]\left[\mathrm{BPh}_{4}\right]$} & DMF & 0 \\
\hline 5 & $\mathrm{Zn}$ & $\mathrm{AgSbF}_{6}$ & DMF & 0 \\
\hline 6 & $\mathrm{Zn}$ & {$\left[\mathrm{Cp}_{2} \mathrm{Fe}\right]\left[\mathrm{BPh}{ }_{4}\right]$} & DMF & 0 \\
\hline 7 & Co & {$\left[\mathrm{Cp}_{2} \mathrm{Fe}\right]\left[\mathrm{BPh}_{4}\right]$} & DMF & 0 \\
\hline 8 & $\mathrm{Mn}$ & {$\left[\mathrm{Cp}_{2} \mathrm{Fe}\right]\left[\mathrm{BPh}_{4}\right]$} & DMF & 0 \\
\hline 9 & $\mathrm{Fe}$ & -- & THF & 0.604 \\
\hline 10 & $\mathrm{Fe}$ & -- & DMF & 0.735 \\
\hline
\end{tabular}

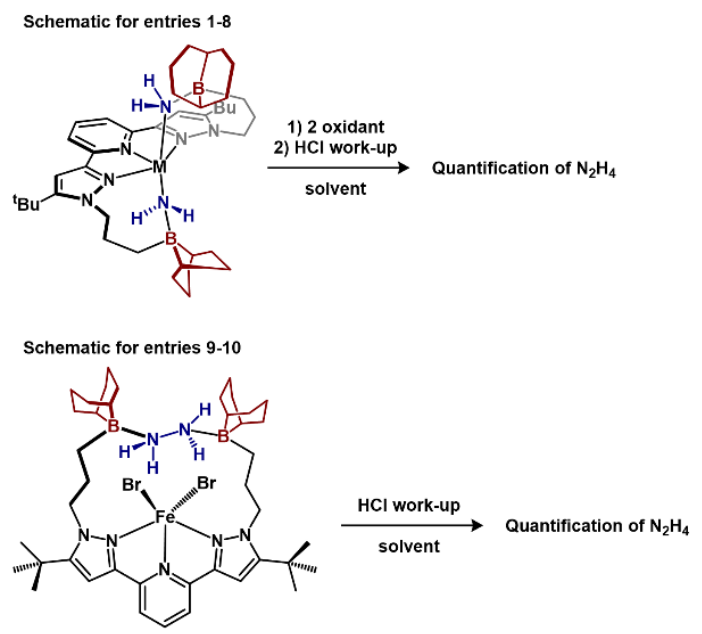

All hydrazine quantification methods were performed following literature procedure. ${ }^{11}$ All chemicals and solvents for the analysis were purchased from commercial vendors and used as received. 


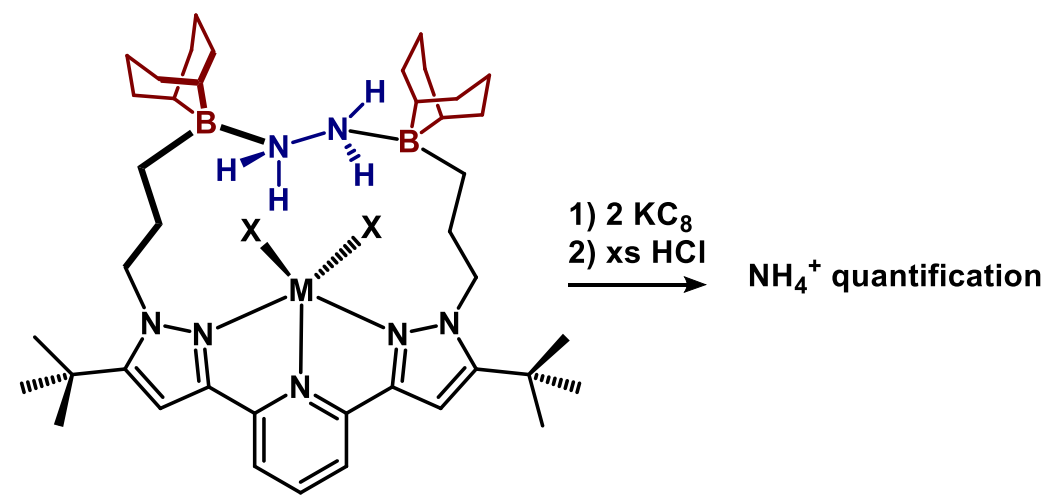

\begin{tabular}{lllllll}
\multicolumn{7}{c}{$\begin{array}{c}\text { Percent } \mathrm{NH}_{4}{ }^{+} \text {detected } \\
\text { Trial Number }\end{array}$} \\
M & 1 & 2 & 3 & 4 & 5 & Average NH$_{4}{ }^{*}$ detected (equivalents) \\
\hline V & 67.09 & 62.77 & 70.25 & - & -- & $1.334+/-0.061$ \\
Mn & 21.79 & 19.32 & 19.85 & 24.04 & -- & $0.425+/-0.037$ \\
$\mathrm{Fe}$ & & & & & & $1.743+/-0.038$ (Previously Reported) \\
$\mathrm{Co}$ & 82.97 & 81.78 & 84.39 & 83.22 & 82.78 & $1.661+/-0.019$ \\
$\mathrm{Zn}$ & & & & & & $0.280+/-0.024$ (Previously Reported) \\
no metal & & & & & & $0.420+/-0.027$ (Previously Reported) \\
\hline
\end{tabular}

*Maximum $100 \%$ assumes $2 \mathrm{NH}_{4}{ }^{+}$per equivalent metal precursor

** Maximum 2 equiv. possible

Details:

All digestion studies are adapted from the procedure of Schrock et al. ${ }^{12}$

Previous results from: J. Am. Chem. Soc. 2017, 139, 18194-18197

General procedure: In a $20 \mathrm{~mL}$ scintillation vial, the metal precursor $(0.019-0.024 \mathrm{mmol}$; sample varied for each run) was dissolved in $5.000 \mathrm{~mL}$ THF and frozen. Upon thawing, potassium graphite (2 equiv.) was added and the reaction stirred $60 \mathrm{~min}$. The vial was removed from the glovebox, and $\mathrm{HCl}(4.0 \mathrm{M}$ in dioxane, 50 equivalents) was added. The mixture was stirred $30 \mathrm{~min}$ and volatiles were removed in vacuo. The material was dissolved in ca. $1.0 \mathrm{~mL}$ dmso- $d_{6}$ and a known quantity of NaBAr${ }_{24}$ (dmso- $d_{6}$ stock solution) was added as internal standard. The solution was filtered, diluted by a factor of 10 , and an ${ }^{1} \mathrm{H}$ NMR spectrum obtained. 


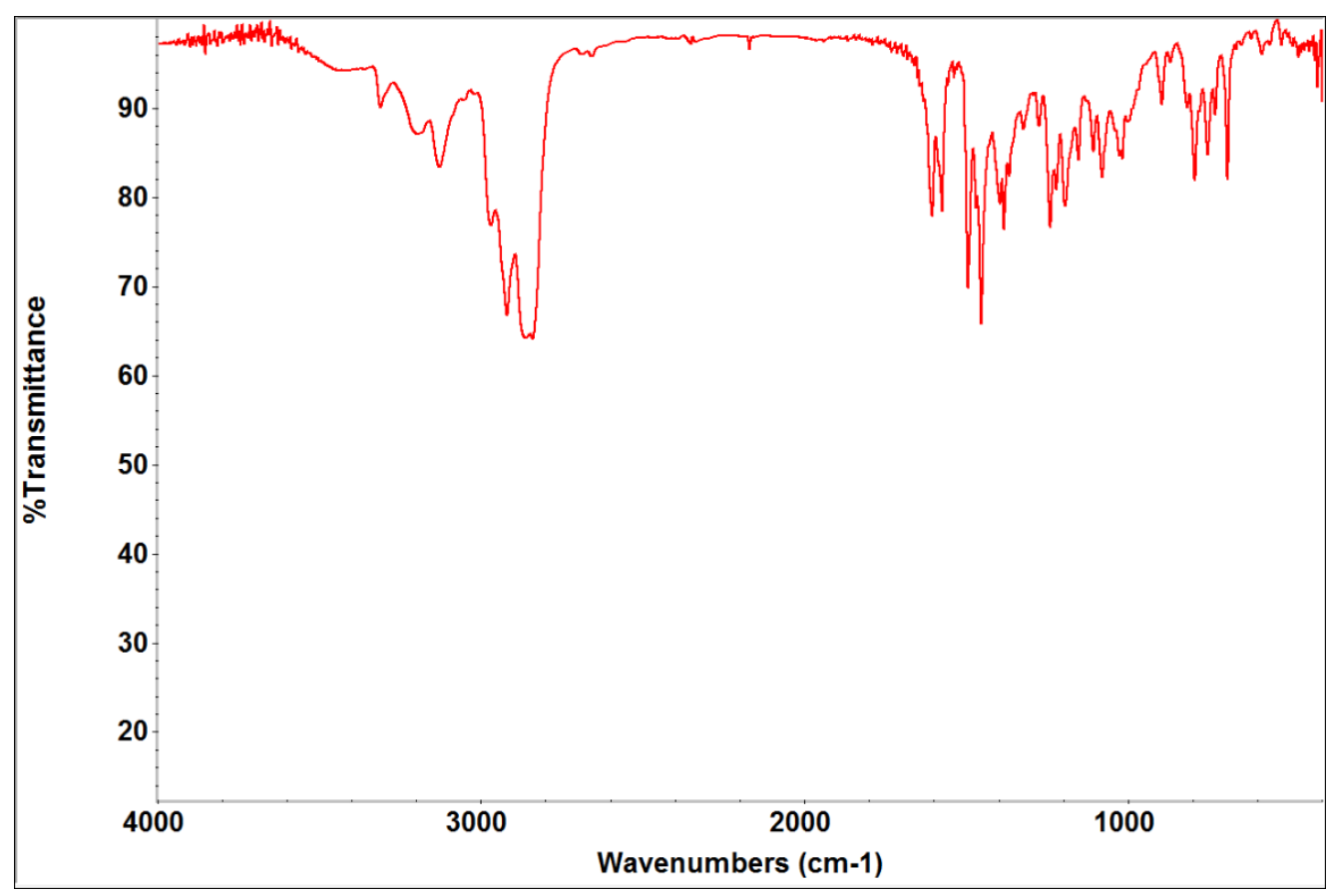

Figure S49 Infrared spectra ( $\mathrm{KBr}$, ambient temperature) of ( $\left.{ }^{\mathrm{BBN} P D P}{ }^{t \mathrm{Bu}}\right) \mathrm{FeBr}_{2}\left(\mathrm{NH}_{2} \mathrm{Ph}\right)_{2}$.

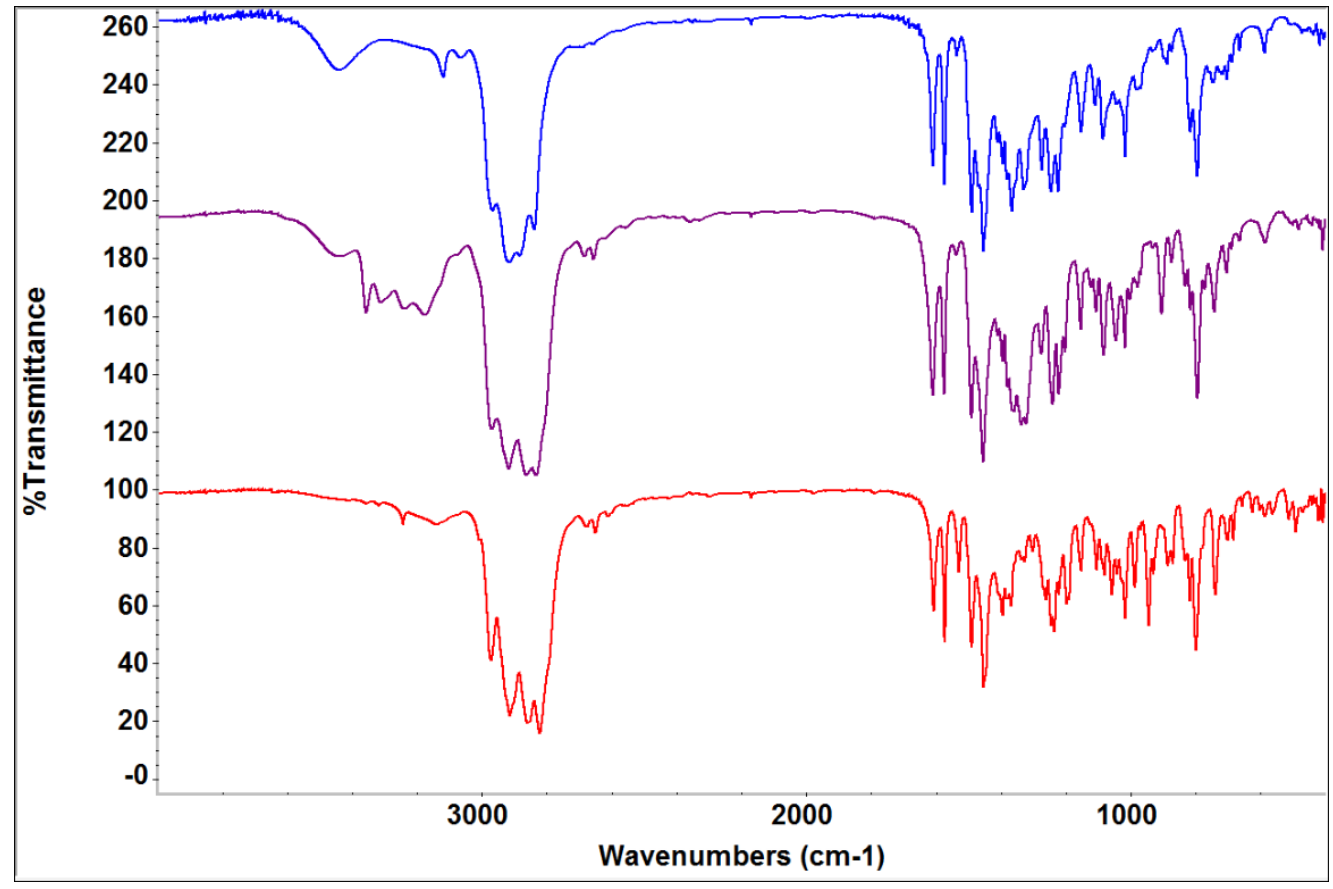

Figure S50 Infrared spectra ( $\mathrm{KBr}$, ambient temperature) of ( $\left.{ }^{\mathrm{BBN} P D P^{t B u}}\right) \mathrm{MnBr}_{2}$ (blue, top), ( $\left.{ }^{B B N P D P}{ }^{t B u}\right) \mathrm{MnBr}_{2}\left(\mathrm{NH}_{3}\right)_{2}$ (purple, middle), and ( $\left.{ }^{B B N} P D P{ }^{t B u}\right) M n\left(N_{2}\right)_{2}$ (red, bottom). 


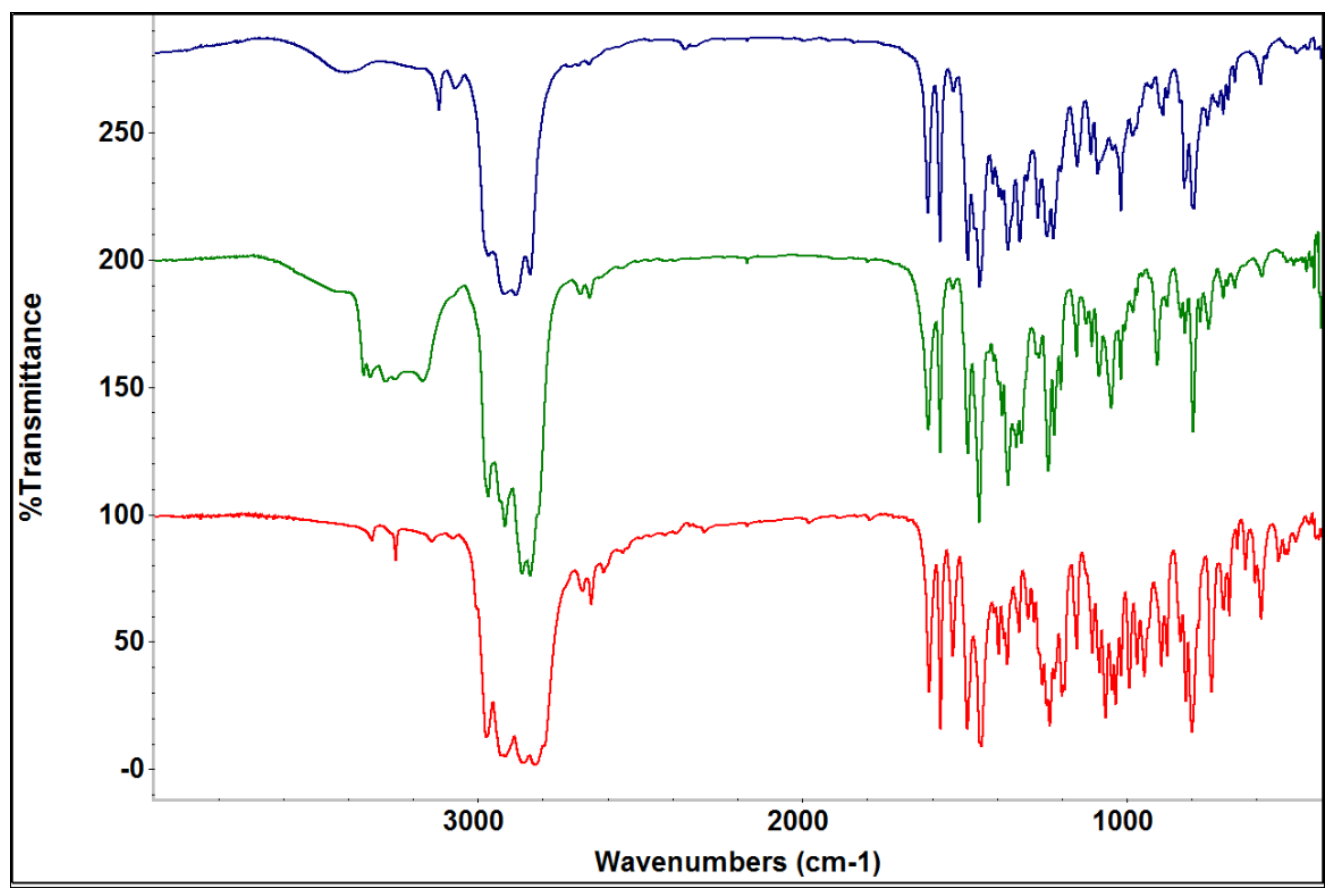

Figure S51 Infrared spectra ( $\mathrm{KBr}$, ambient temperature) of ( $\left.{ }^{\mathrm{BBN} P D P}{ }^{\mathrm{tBu}}\right) \mathrm{ZnCl}_{2}$ (blue, top), ( $\left.{ }^{\mathrm{BBN} P D P}{ }^{\mathrm{BBu}}\right) \mathrm{ZnCl}_{2}\left(\mathrm{NH}_{3}\right)_{2}$ (green, middle), and ( $\left.{ }^{\mathrm{BBN} P D P} \mathrm{P}^{\mathrm{BBu}}\right) \mathrm{Zn}\left(\mathrm{NH}_{2}\right)_{2}$ (red, bottom). The data for

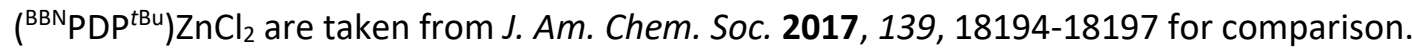

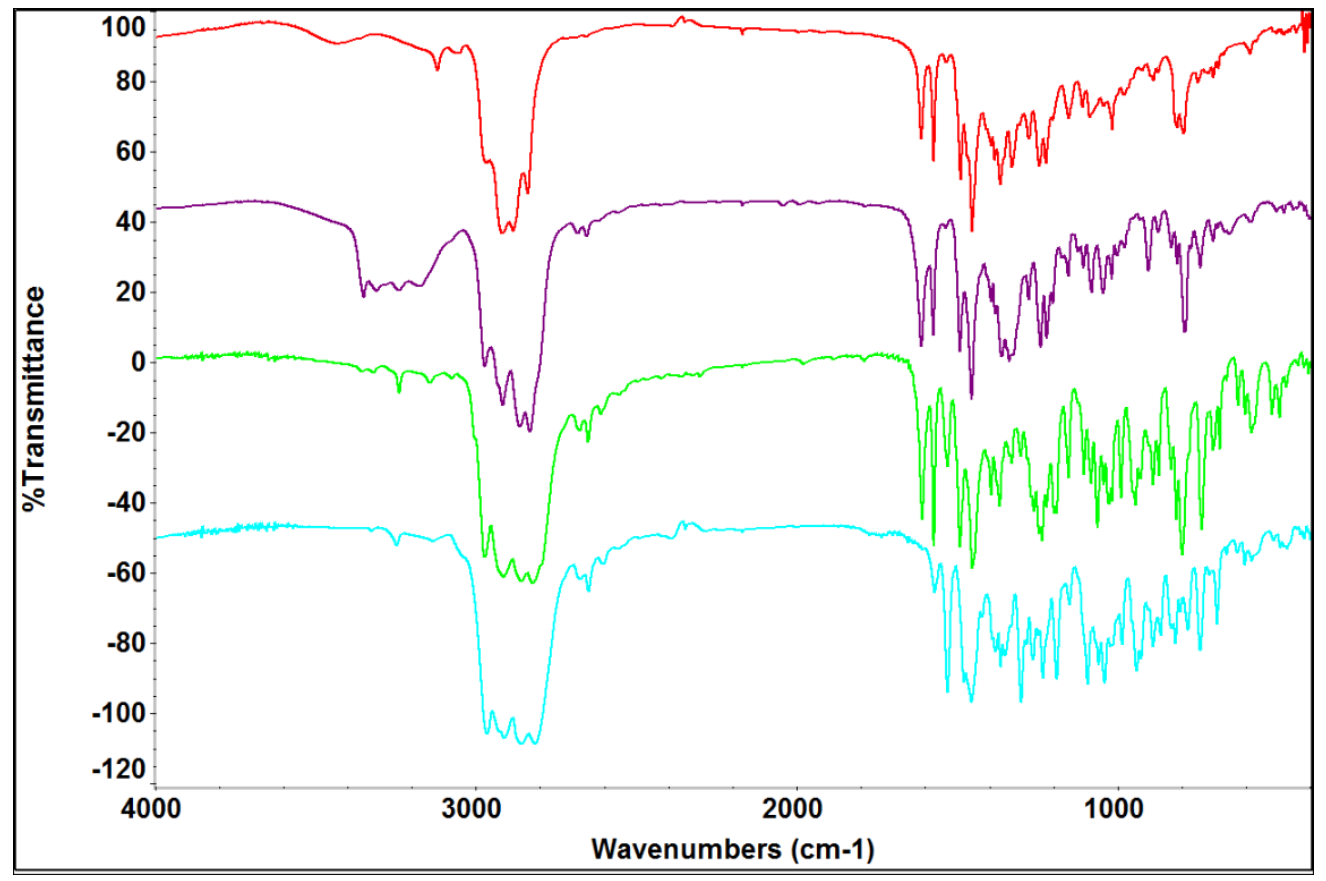

Figure S52 Infrared spectra ( $\mathrm{KBr}$, ambient temperature) of ( $\left.{ }^{\mathrm{BBN} P D P}{ }^{\mathrm{BBu}}\right) \mathrm{CoBr}_{2}$ (red, top), $\left({ }^{\mathrm{BBN} P D P} \mathrm{P}^{\mathrm{tBu}}\right) \mathrm{COBr}_{2}\left(\mathrm{NH}_{3}\right)_{2}$ (purple, middle), ( $\left.{ }^{\mathrm{BBN}} \mathrm{PDP}{ }^{\mathrm{BBu}}\right) \mathrm{Co}\left(\mathrm{NH}_{2}\right)_{2}$ (green, middle), and $\left[\mathrm{Bu}_{4} \mathrm{~N}\right]\left[\left({ }^{\mathrm{BBN} P D P}{ }^{\mathrm{BB} u}\right) \mathrm{Co}\left(\mathrm{NH}_{2}\right)_{2}\right]$ (teal, bottom). 


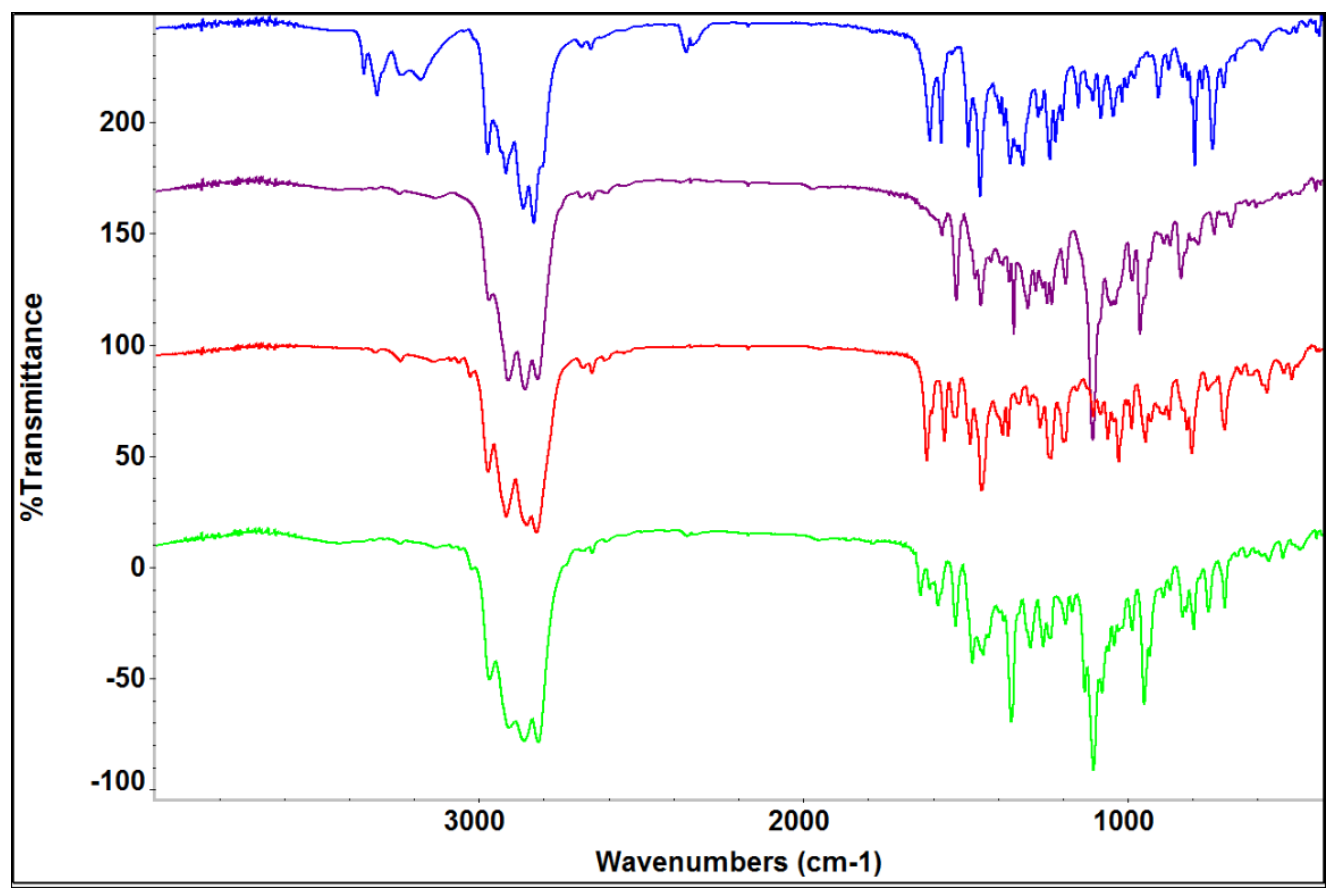

Figure S53 Infrared spectra ( $\mathrm{KBr}$, ambient temperature) of ( $\left.{ }^{\mathrm{BBN} P D P}{ }^{\mathrm{tBu}}\right) \mathrm{FeBr}_{2}\left(\mathrm{NH}_{3}\right)_{2}$ (blue, top), [K(2,2,2crypt)][(benzyl-- $\left.{ }^{\mathrm{BN}} \mathrm{PDP}{ }^{\mathrm{tBu}}\right) \mathrm{Fe}\left(\mathrm{NH}_{2}\right)_{2}$ ] (purple, middle), (benzyl- $\left.{ }^{\mathrm{BBN}} \mathrm{PDP}{ }^{\mathrm{tBu}}\right) \mathrm{Fe}\left(\mathrm{NH}_{2}\right)_{2}$ (middle, red) and $[\mathrm{K}(2,2,2$-crypt $)]\left[\left(^{\mathrm{BBN}} \mathrm{PDP} \mathrm{tBu}^{\mathrm{Bu}}\right) \mathrm{Fe}\left(\mathrm{NH}_{2}\right)_{2}\right]$ (green, bottom).

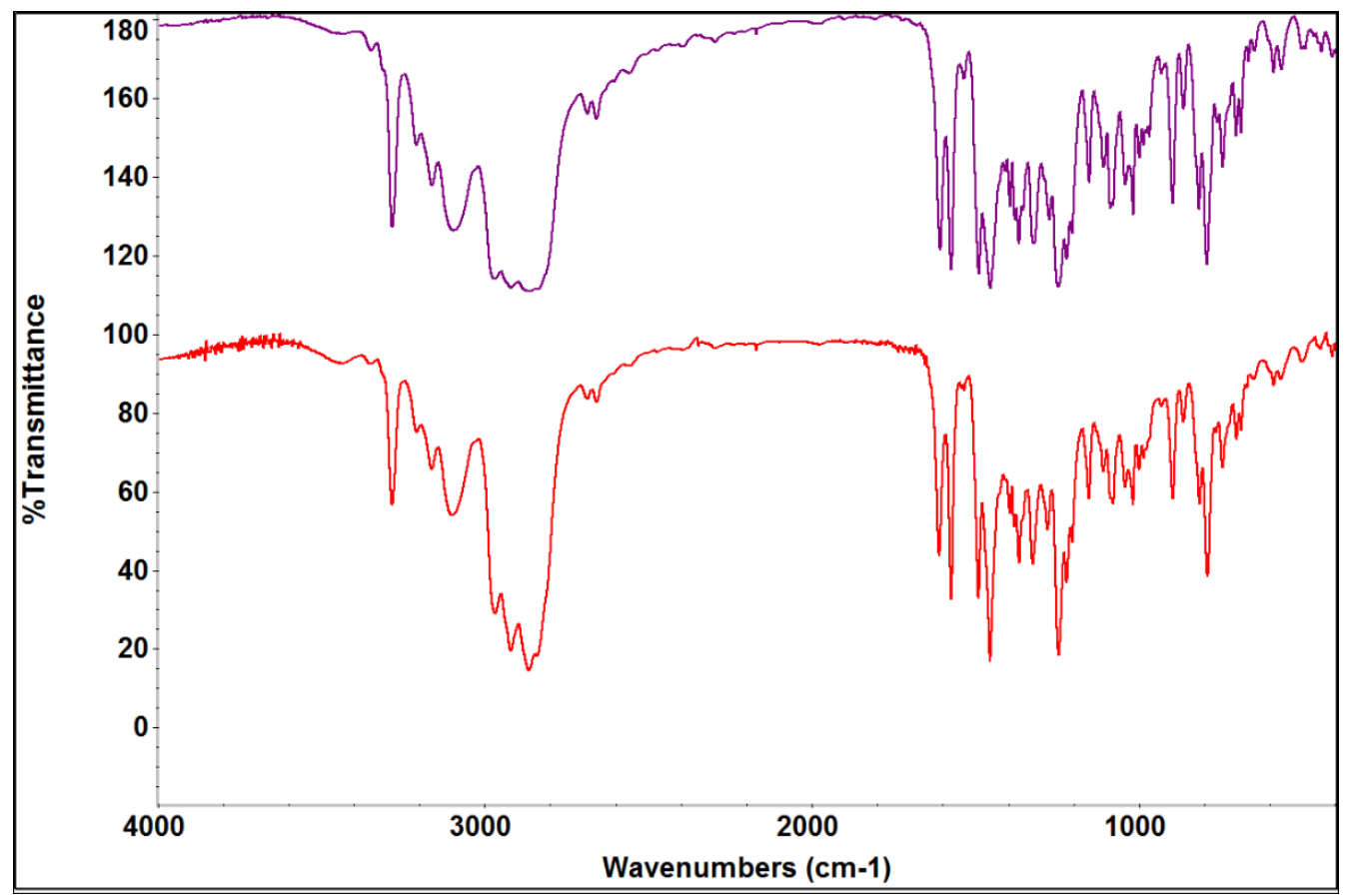

Figure S54 Infrared spectra ( $\mathrm{KBr}$, ambient temperature) of ( $\left.{ }^{\mathrm{BBN} P D P}{ }^{\mathrm{BBu}}\right) \mathrm{MnBr}_{2}\left(\mathrm{~N}_{2} \mathrm{H}_{4}\right)$ (purple, top) and $\left({ }^{\mathrm{BBN} P D P} \mathrm{PBu}^{\mathrm{BB}}\right) \mathrm{CoBr}_{2}\left(\mathrm{~N}_{2} \mathrm{H}_{4}\right)$ (red, bottom). 


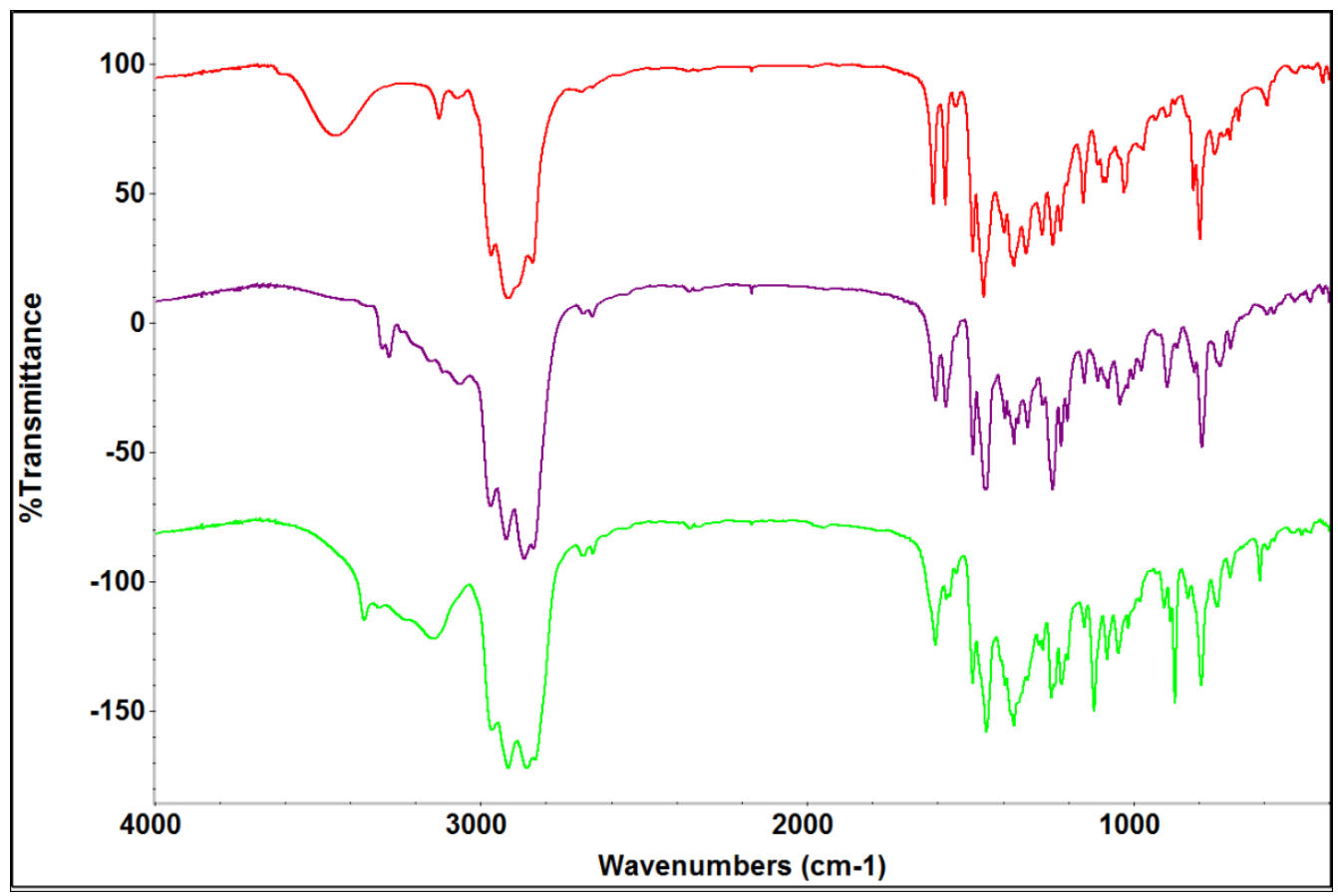

Figure S55 Infrared spectra ( $\mathrm{KBr}$, ambient temperature) of ( $\left.{ }^{\mathrm{BBN}} \mathrm{PDP}^{\mathrm{tBu}}\right) \mathrm{VCl}_{3}$ (red, top),

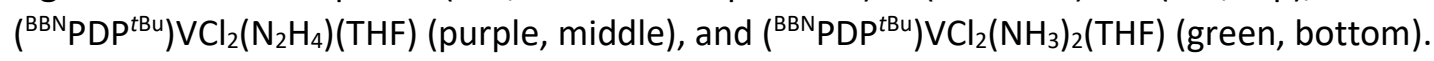

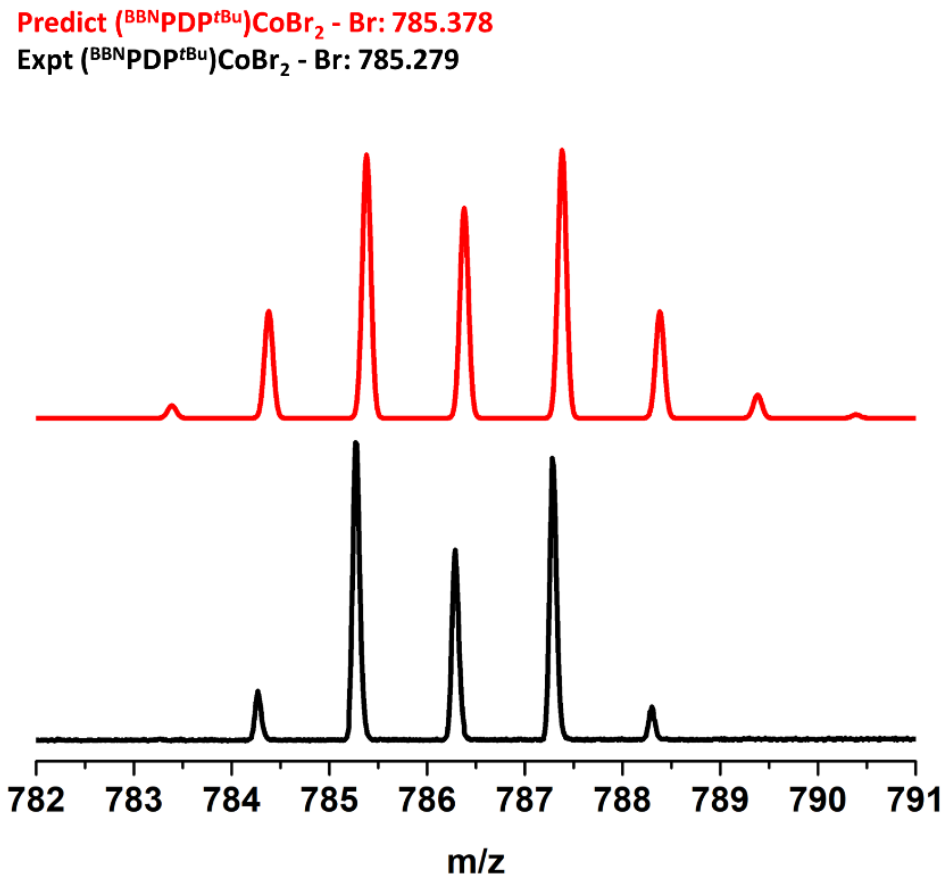

Figure S56 MALDI-TOF spectrum of ( $\left.{ }^{\mathrm{BBN}} \mathrm{PDP}^{\mathrm{BB}}\right) \mathrm{CoBr}_{2}$ (bottom, black) obtained in an anthracene matrix and the predicted isotopic pattern (top, red). 
Predict ( ${ }^{B B N}$ PDP $\left.{ }^{\mathrm{B} u}\right) \mathrm{MnBr}_{2}-\mathrm{Br}: 781.383$

Expt ( $\left.{ }^{B B N P D P}{ }^{B u}\right) \mathrm{MnBr}_{2}-\mathrm{Br}: 781.236$

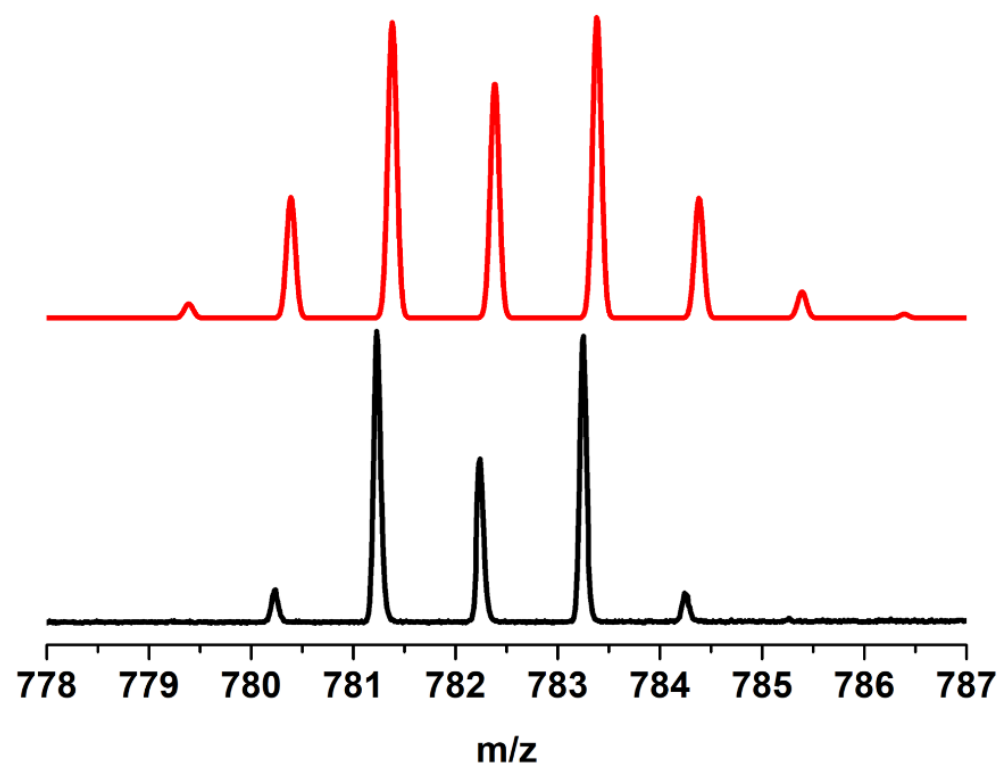

Figure S57 MALDI-TOF spectrum of ( $\left.{ }^{\mathrm{BBN}} \mathrm{PDP}{ }^{\mathrm{tBu}}\right) \mathrm{MnBr}_{2}$ (bottom, black) obtained in an anthracene matrix and the predicted isotopic pattern (top, red).

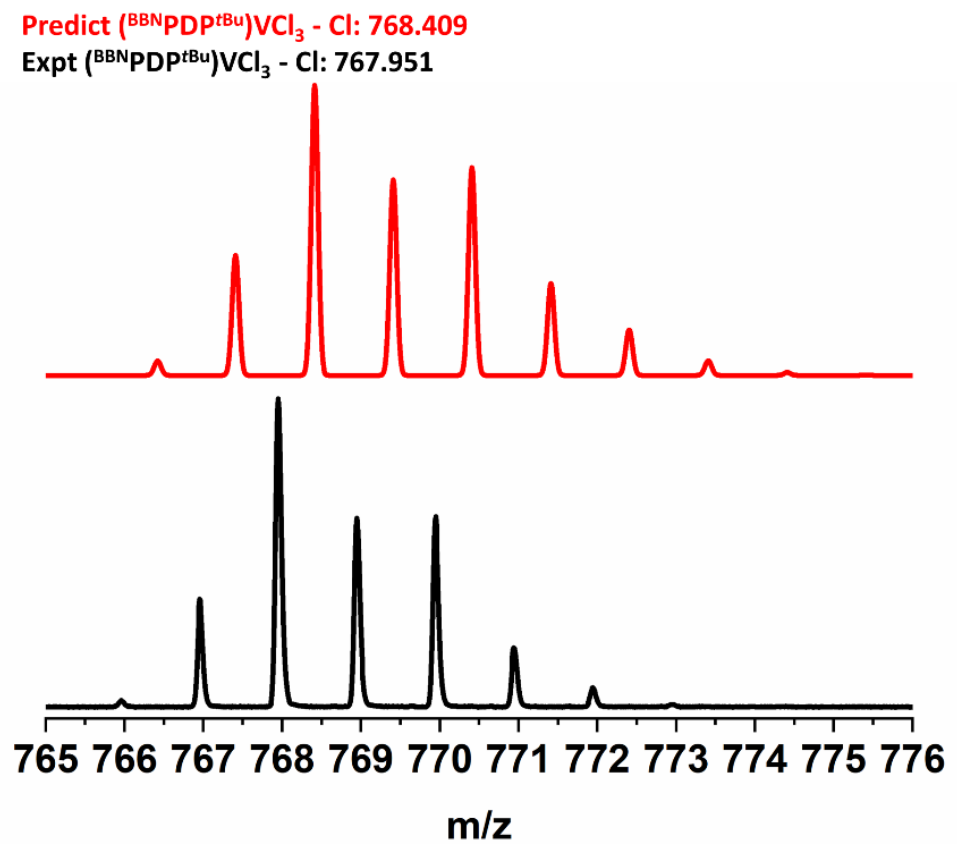

Figure S58 MALDI-TOF spectrum of ( $\left.{ }^{\mathrm{BBN}} \mathrm{PDP}^{\mathrm{tBu}}\right) \mathrm{VCl}_{3}$ (bottom, black) obtained in an anthracene matrix and the predicted isotopic pattern (top, red). 


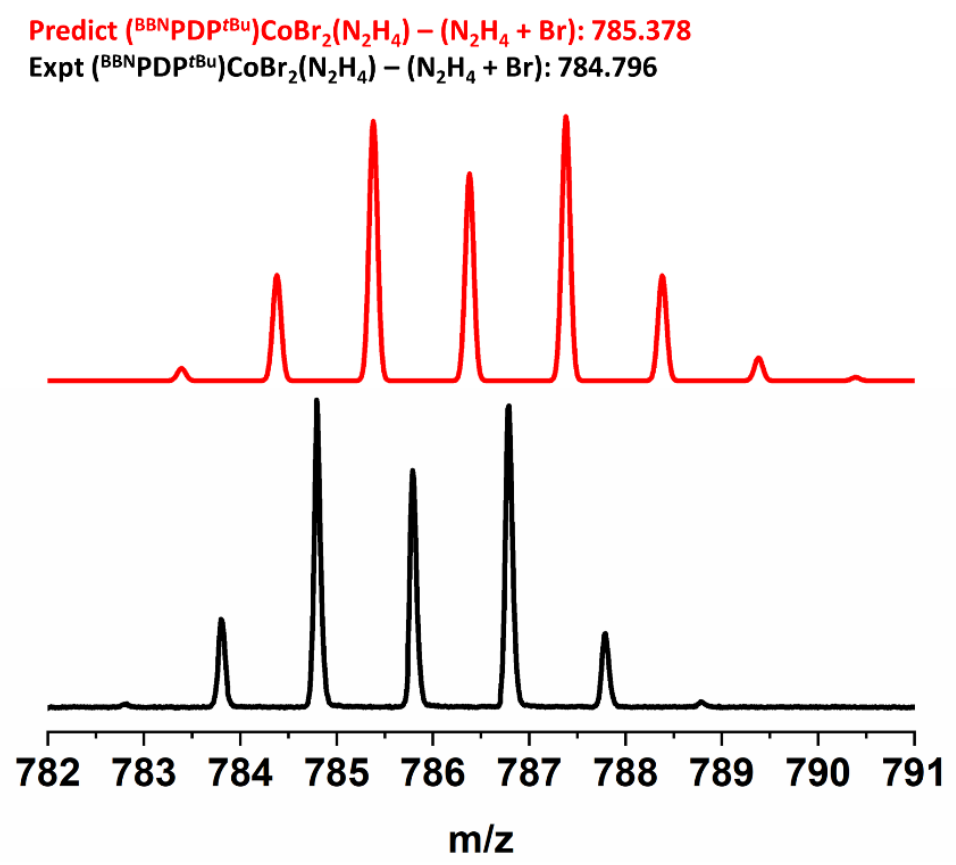

Figure S59 MALDI-TOF spectrum of ( $\left.{ }^{B B N P D P}{ }^{\text {BBu}}\right) \mathrm{CoBr}_{2}\left(\mathrm{~N}_{2} \mathrm{H}_{4}\right)$ (bottom, black) obtained in an anthracene matrix and the predicted isotopic pattern (top, red).

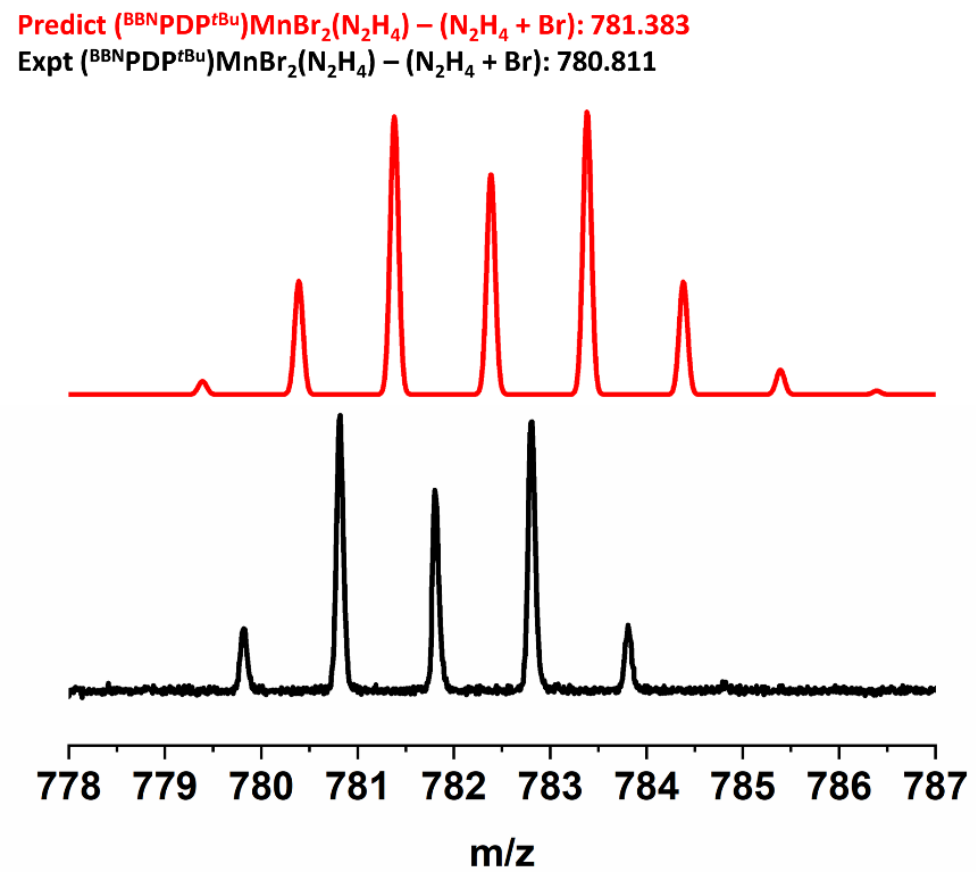

Figure S60 MALDI-TOF spectrum of ( $\left.{ }^{\mathrm{BBN} P D P}{ }^{\mathrm{BBu}}\right) \mathrm{MnBr}_{2}\left(\mathrm{~N}_{2} \mathrm{H}_{4}\right)$ (bottom, black) obtained in an anthracene matrix and the predicted isotopic pattern (top, red). 


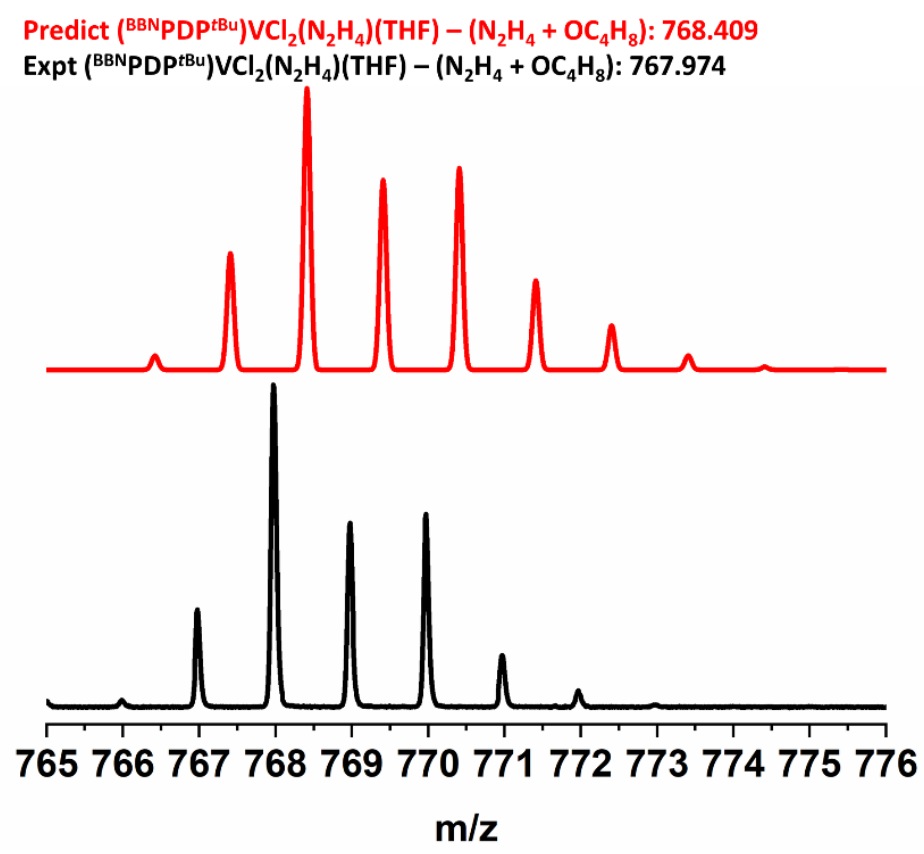

Figure S61 MALDI-TOF spectrum of ( $\left.{ }^{\mathrm{BBN} P D P}{ }^{\mathrm{BBu}}\right) \mathrm{VCl}_{2}\left(\mathrm{~N}_{2} \mathrm{H}_{4}\right)(\mathrm{THF})$ (bottom, black) obtained in an anthracene matrix and the predicted isotopic pattern (top, red).

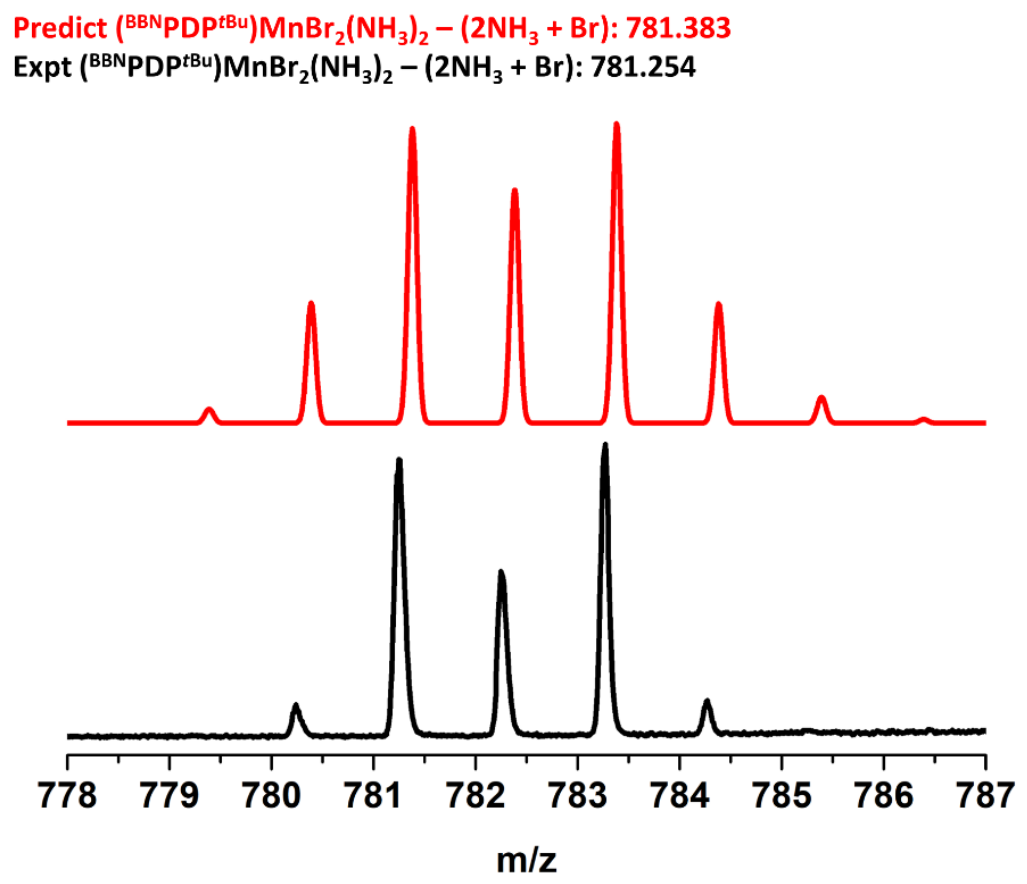

Figure S62 MALDI-TOF spectrum of ( $\left.{ }^{\mathrm{BBN} P D P}{ }^{\mathrm{BBu}}\right) \mathrm{MnBr}_{2}\left(\mathrm{NH}_{3}\right)_{2}$ (bottom, black) obtained in an anthracene matrix and the predicted isotopic pattern (top, red). 
Predict (BBNPDPtBu) $\mathrm{ZnCl}_{2}\left(\mathrm{NH}_{3}\right)_{2}-\left(2 \mathrm{NH}_{3}+\mathrm{Cl}\right): 746.425$

Expt ( $\left.{ }^{\mathrm{BBN} P D P}{ }^{\mathrm{Bu}}\right) \mathrm{ZnCl}_{2}\left(\mathrm{NH}_{3}\right)_{2}-\left(2 \mathrm{NH}_{3}+\mathrm{Cl}\right): 746.314$

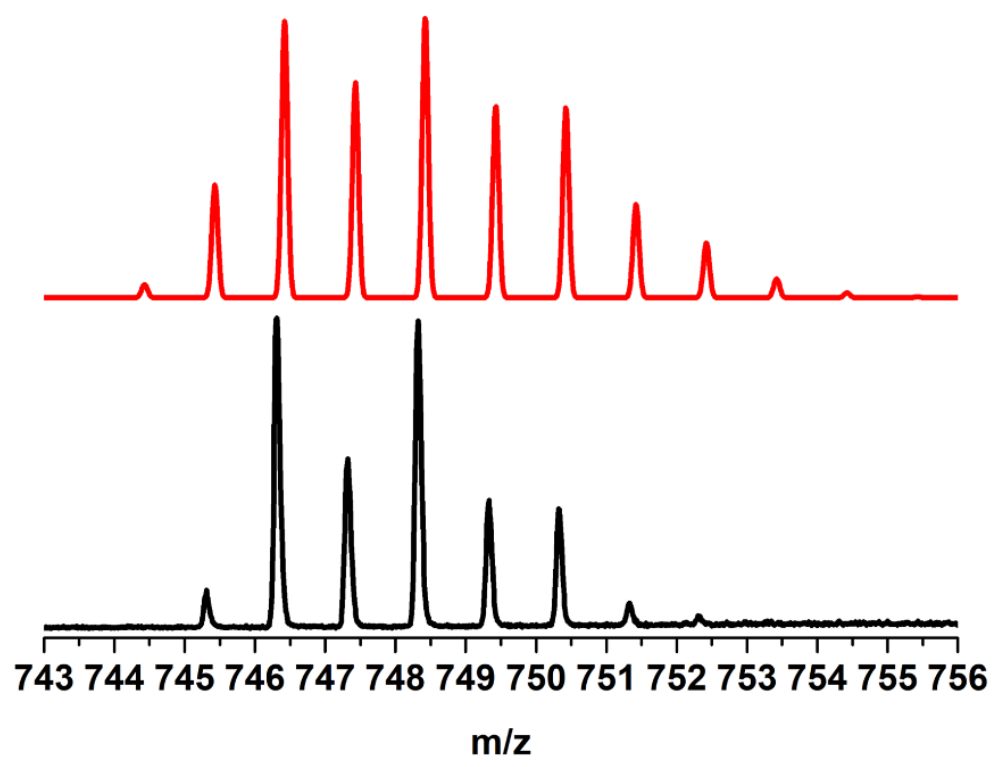

Figure S63 MALDI-TOF spectrum of ( $\left.{ }^{\mathrm{BBN} P D P}{ }^{\mathrm{tBu}}\right) \mathrm{ZnCl}_{2}\left(\mathrm{NH}_{3}\right)_{2}$ (bottom, black) obtained in an anthracene matrix and the predicted isotopic pattern (top, red).

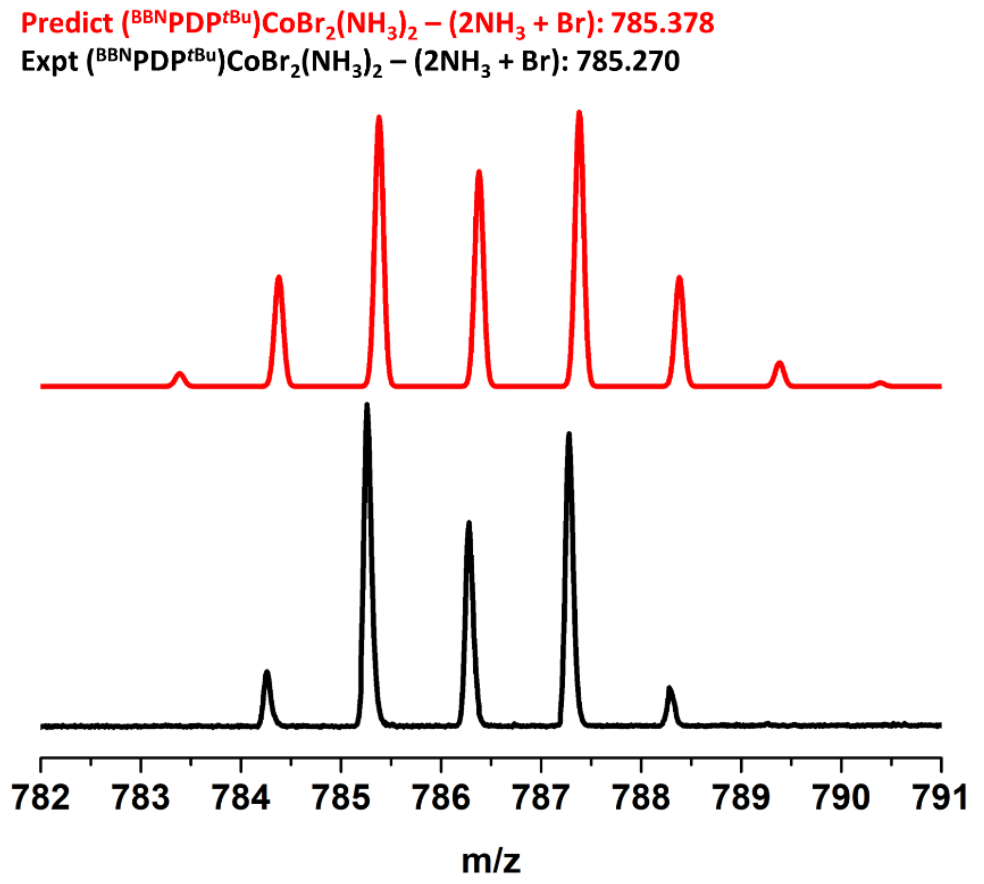

Figure S64 MALDI-TOF spectrum of ( $\left.{ }^{\mathrm{BBN} P D P}{ }^{\mathrm{BBu}}\right) \mathrm{CoBr}_{2}\left(\mathrm{NH}_{3}\right)_{2}$ (bottom, black) obtained in an anthracene matrix and the predicted isotopic pattern (top, red). 


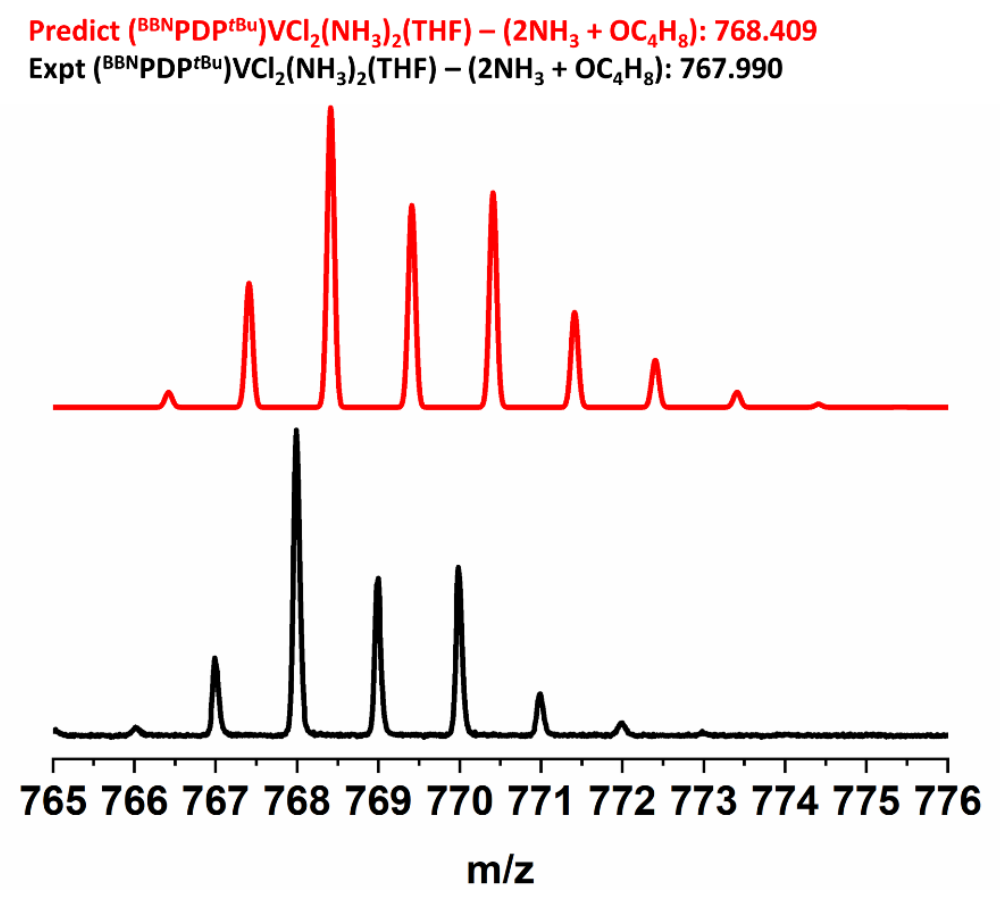

Figure $\mathrm{S} 65 \mathrm{MALDI}-\mathrm{TOF}$ spectrum of $\left({ }^{\mathrm{BBN} P D P}{ }^{\mathrm{tBu}}\right) \mathrm{VCl}_{2}\left(\mathrm{NH}_{3}\right)_{2}$ (THF) (bottom, black) obtained in an anthracene matrix and the predicted isotopic pattern (top, red).

Predict (BBNPDPtBu) $\mathrm{FeBr}_{2}\left(\mathrm{NH}_{3}\right)_{2}-\left(2 \mathrm{NH}_{3}\right): 863.248$

Expt ( $\left.{ }^{\mathrm{BBN} P D P}{ }^{\mathrm{BBu}}\right) \mathrm{FeBr}_{2}\left(\mathrm{NH}_{3}\right)_{2}-\left(2 \mathrm{NH}_{3}\right): 863.346$

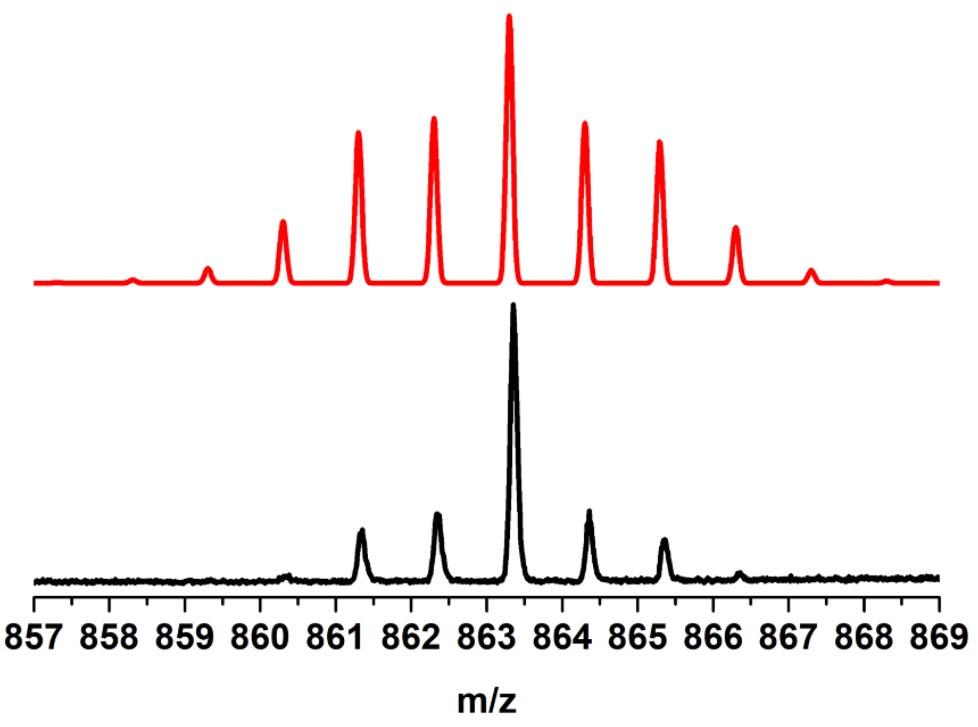

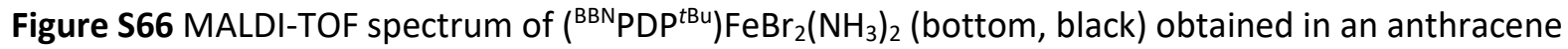
matrix and the predicted isotopic pattern (top, red). 


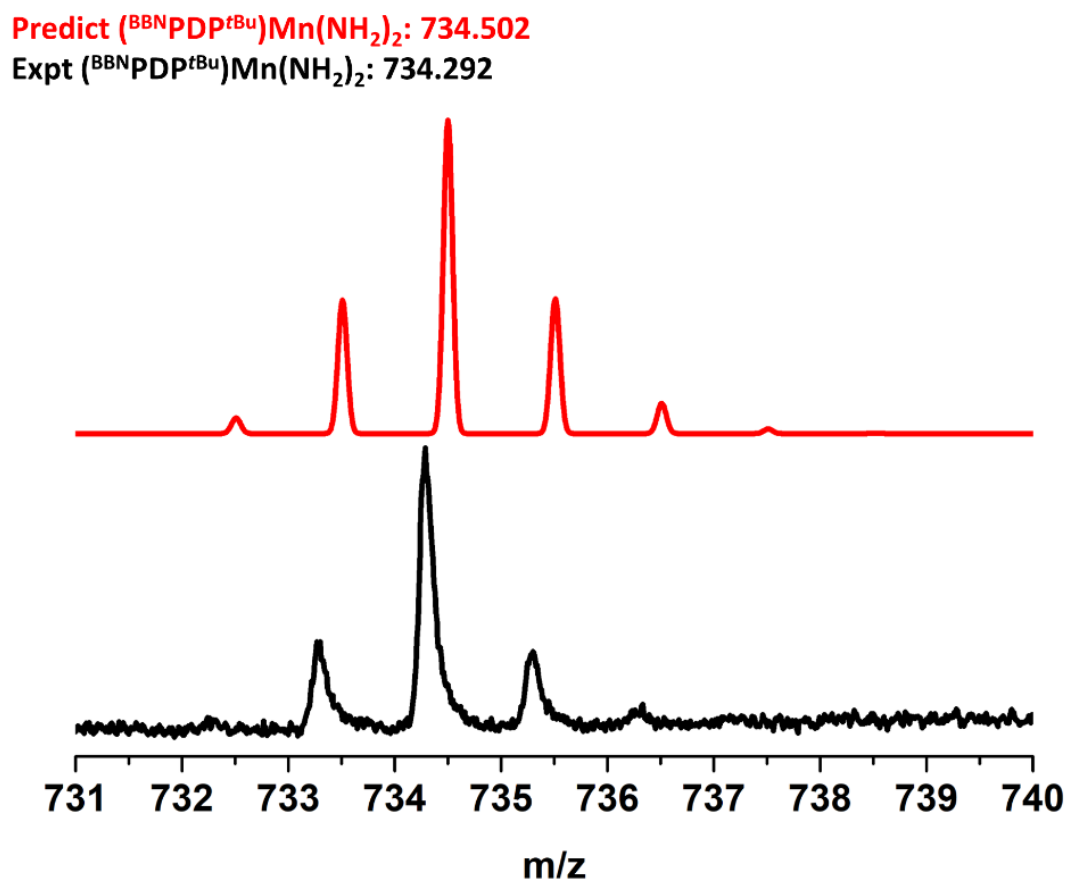

Figure S67 MALDI-TOF spectrum of ( $\left.{ }^{\mathrm{BBN} P D P}{ }^{\mathrm{BBu}}\right) \mathrm{Mn}\left(\mathrm{NH}_{2}\right)_{2}$ (bottom, black) obtained in an anthracene matrix and the predicted isotopic pattern (top, red).

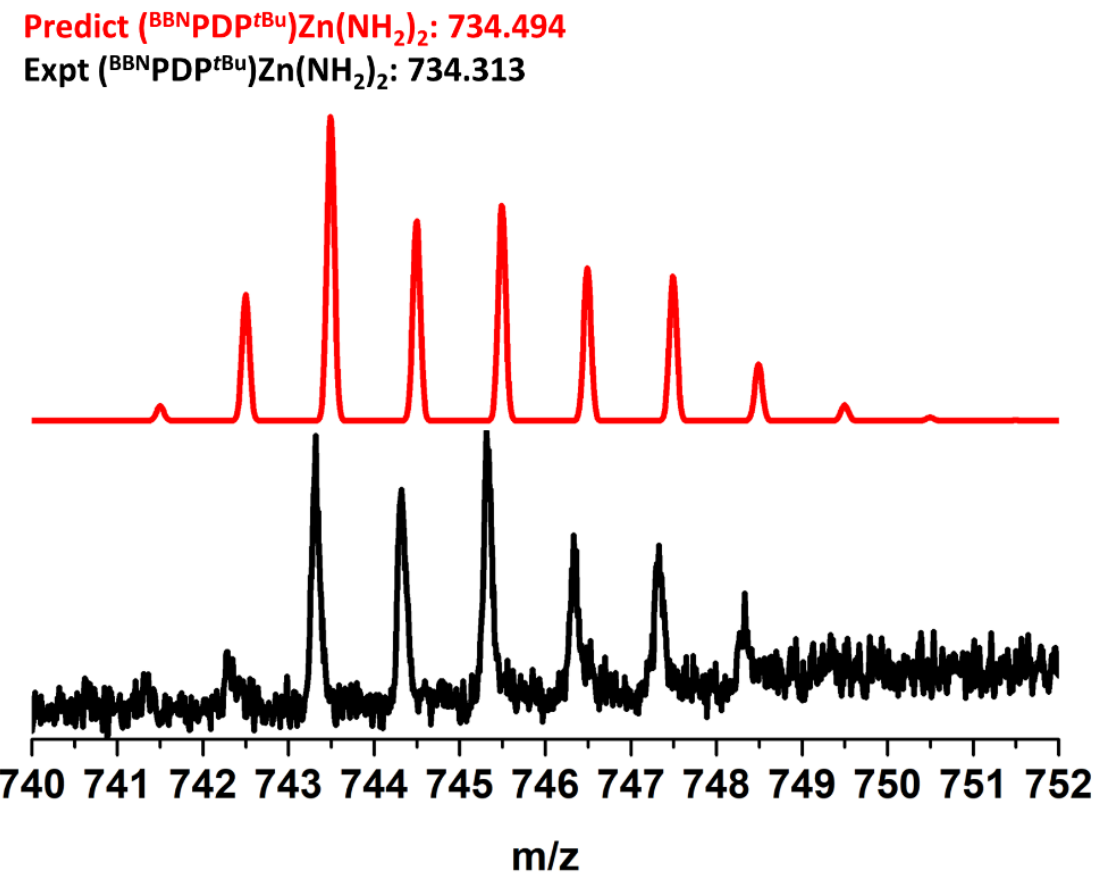

Figure S68 MALDI-TOF spectrum of ( $\left.{ }^{\mathrm{BBN}} \mathrm{PDP}{ }^{\mathrm{BBu}}\right) \mathrm{Zn}\left(\mathrm{NH}_{2}\right)_{2}$ (bottom, black) obtained in an anthracene matrix and the predicted isotopic pattern (top, red). 


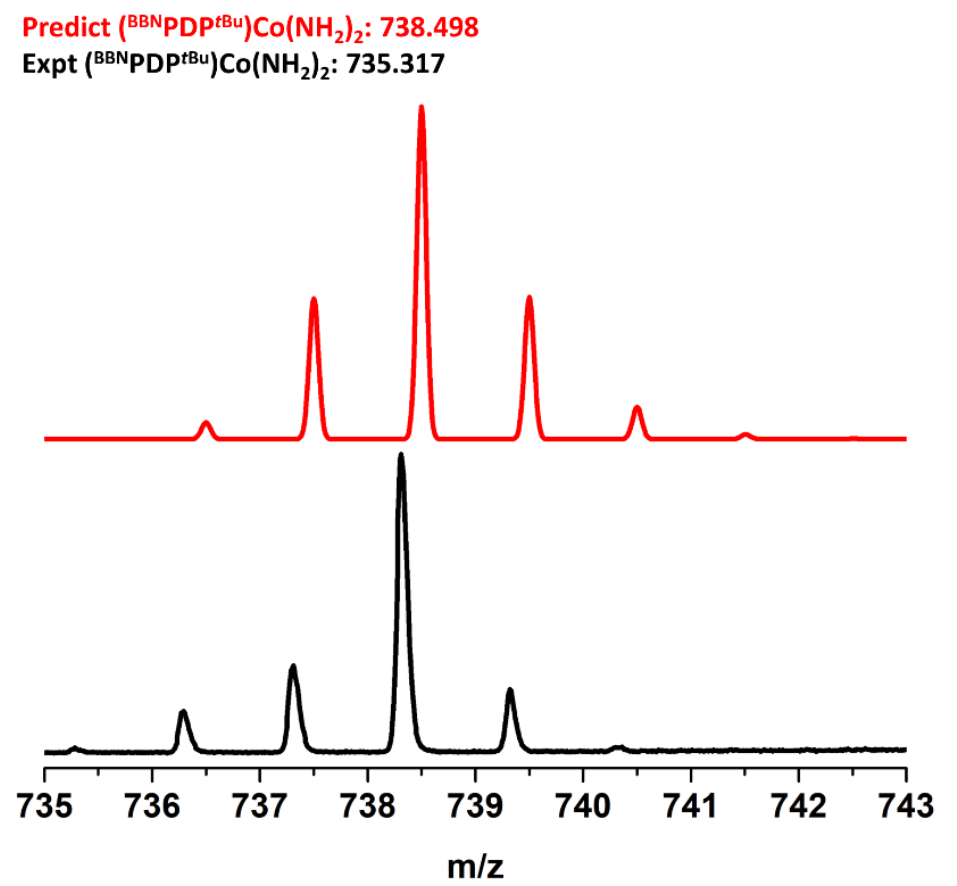

Figure S69 MALDI-TOF spectrum of $\left({ }^{\mathrm{BBN}} \mathrm{PDP}{ }^{\mathrm{tBu}}\right) \mathrm{Co}\left(\mathrm{NH}_{2}\right)_{2}$ (bottom, black) obtained in an anthracene matrix and the predicted isotopic pattern (top, red).

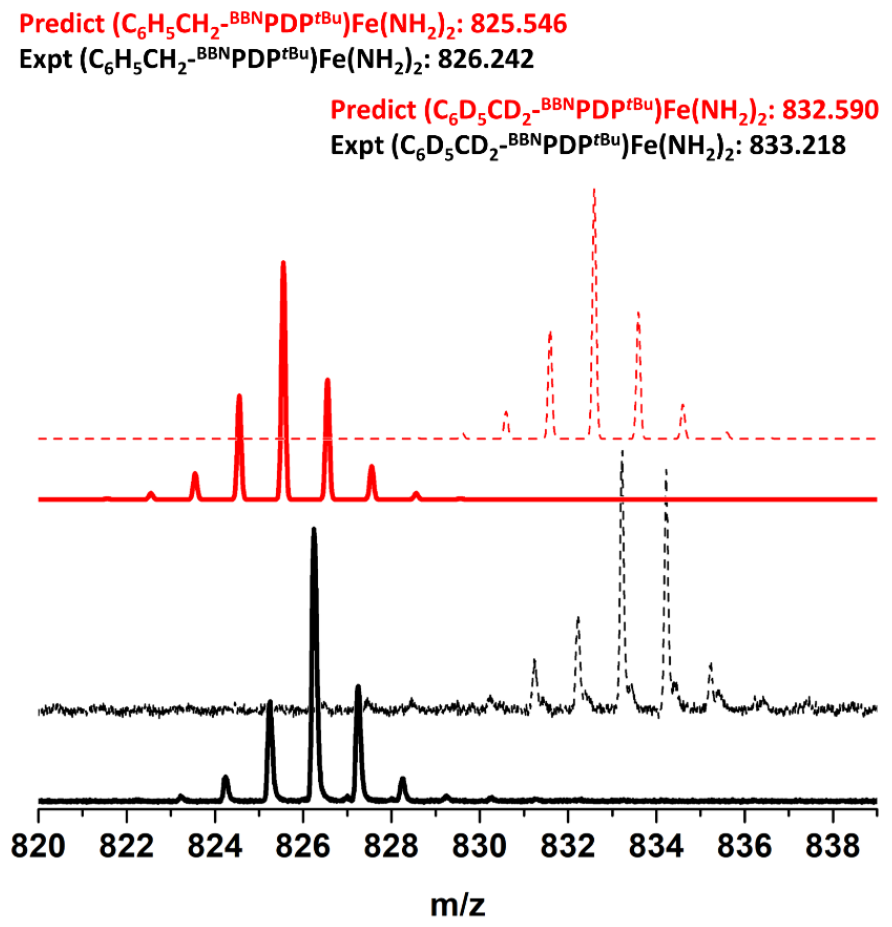

Figure S70 MALDI-TOF spectrum of (benzyl-- $\left.{ }^{\mathrm{BBN}} \mathrm{PDP}{ }^{\mathrm{tBu}}\right) \mathrm{Fe}\left(\mathrm{NH}_{2}\right)_{2}$ (bottom, black) obtained in an anthracene matrix and the predicted isotopic pattern (top, red). The dashed-line spectra are for the deuterated analogue, (benzyl- $\left.d_{7^{-}}{ }^{\mathrm{BBN}} \mathrm{PDP}{ }^{\mathrm{tBu}}\right) \mathrm{Fe}\left(\mathrm{NH}_{2}\right)_{2}$. 

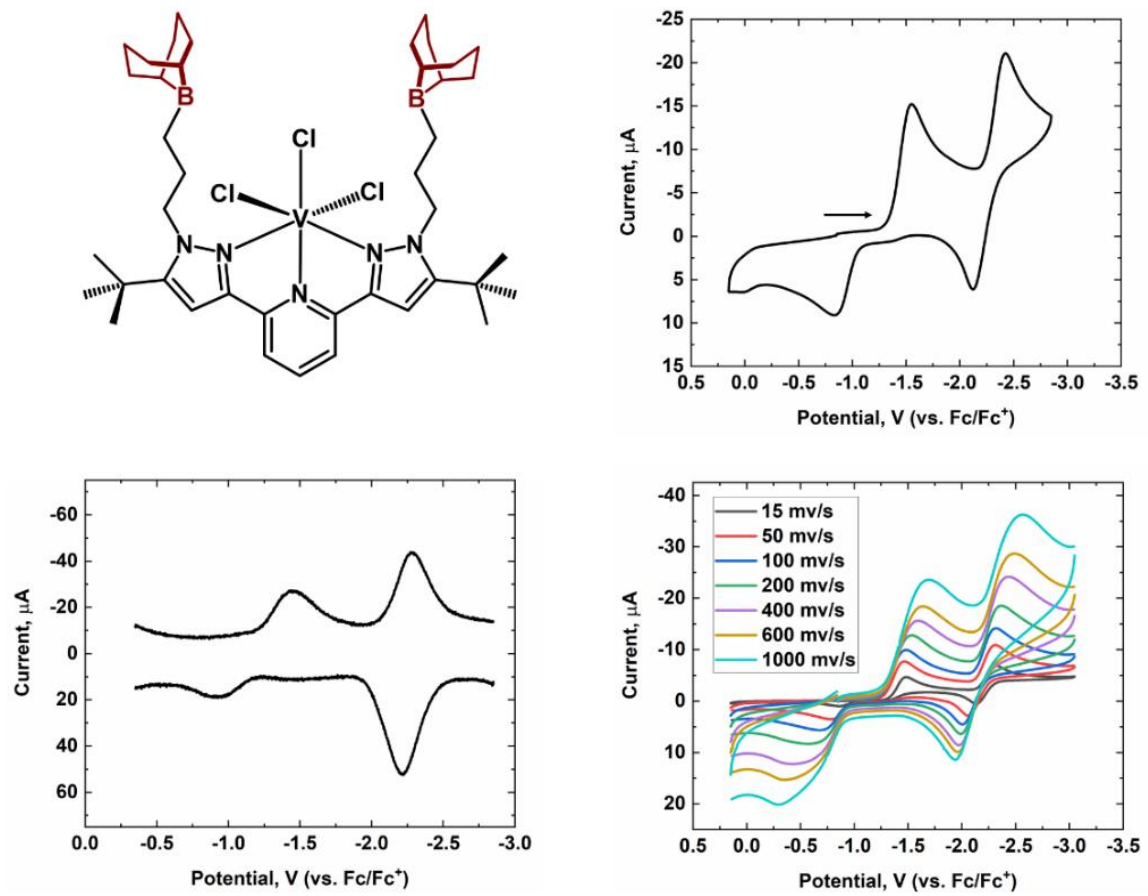

Figure S71 Electrochemical data for $\left({ }^{\mathrm{BBN}} \mathrm{PDP}^{\mathrm{BBu}}\right) \mathrm{VCl}_{3}(0.8 \mathrm{mM})$ recorded in THF with $0.2 \mathrm{M}\left[\mathrm{Bu}_{4} \mathrm{~N}\right]\left[\mathrm{PF}_{6}\right]$. Working electrode: glassy carbon; counter electrode: platinum; reference electrode: silver wire. Top: full scan. Bottom: square wave voltammogram and scan rate dependence.
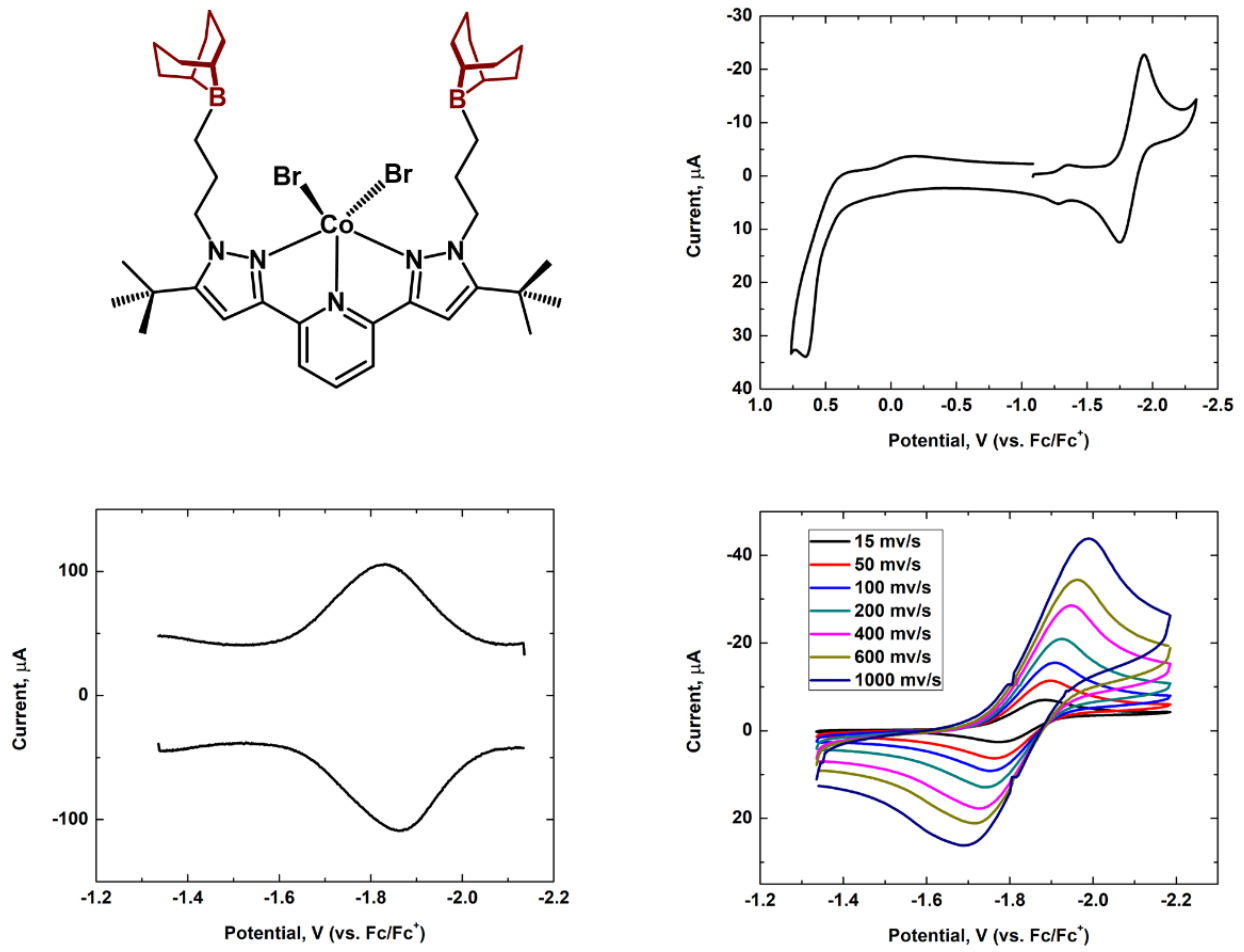

Figure S72 Electrochemical data for ( $\left.{ }^{\mathrm{BBN} P D P^{t B u}}\right) \mathrm{CoBr}_{2}(0.8 \mathrm{mM})$ recorded in THF with $0.2 \mathrm{M}\left[\mathrm{Bu}_{4} \mathrm{~N}\right]\left[\mathrm{PF}_{6}\right]$. Working electrode: glassy carbon; counter electrode: platinum; reference electrode: silver wire. Top: full scan. Bottom: square wave voltammogram and scan rate dependence. 

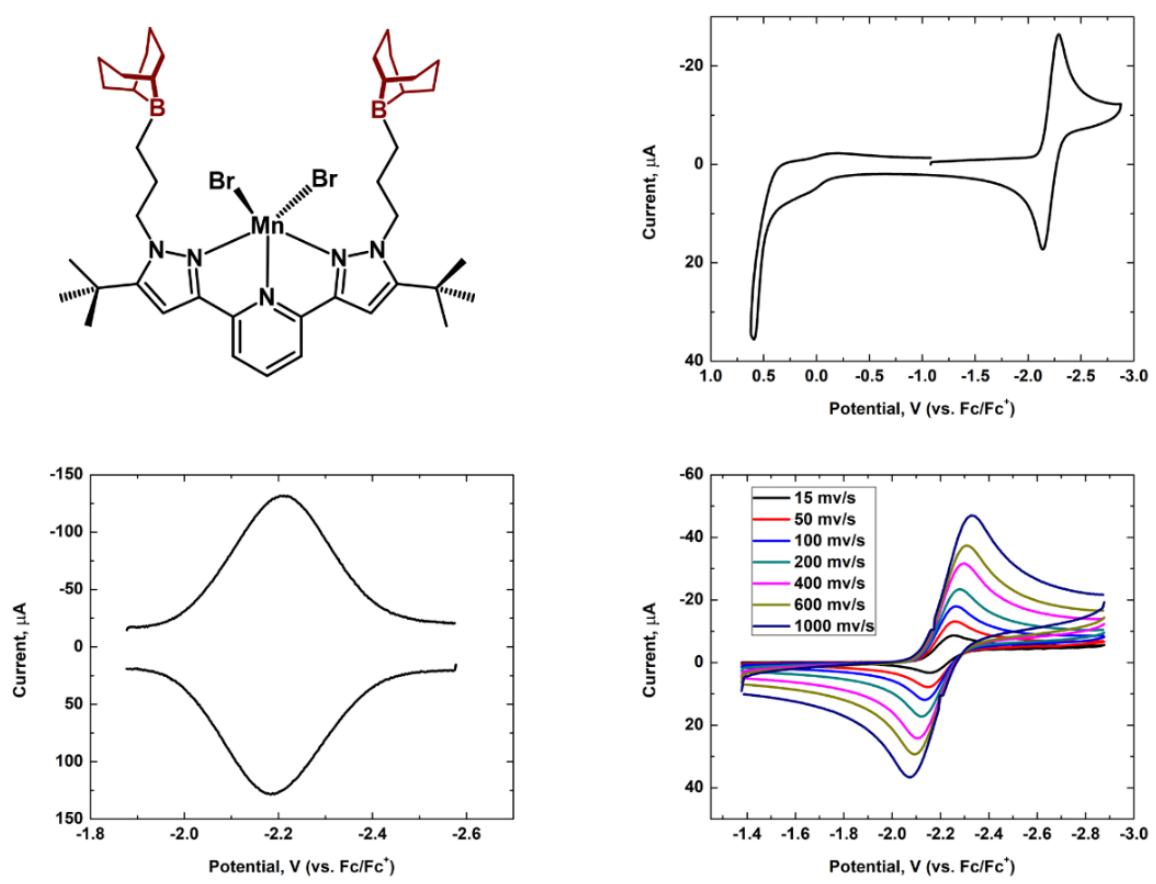

Figure S73 Electrochemical data for $\left({ }^{\mathrm{BBN}} \mathrm{PDP}^{\mathrm{BBu}}\right) \mathrm{MnBr}_{2}(0.8 \mathrm{mM})$ recorded in THF with $0.2 \mathrm{M}\left[\mathrm{Bu}_{4} \mathrm{~N}\right]\left[\mathrm{PF}_{6}\right]$. Working electrode: glassy carbon; counter electrode: platinum; reference electrode: silver wire. Top: full scan. Bottom: square wave voltammogram and scan rate dependence.
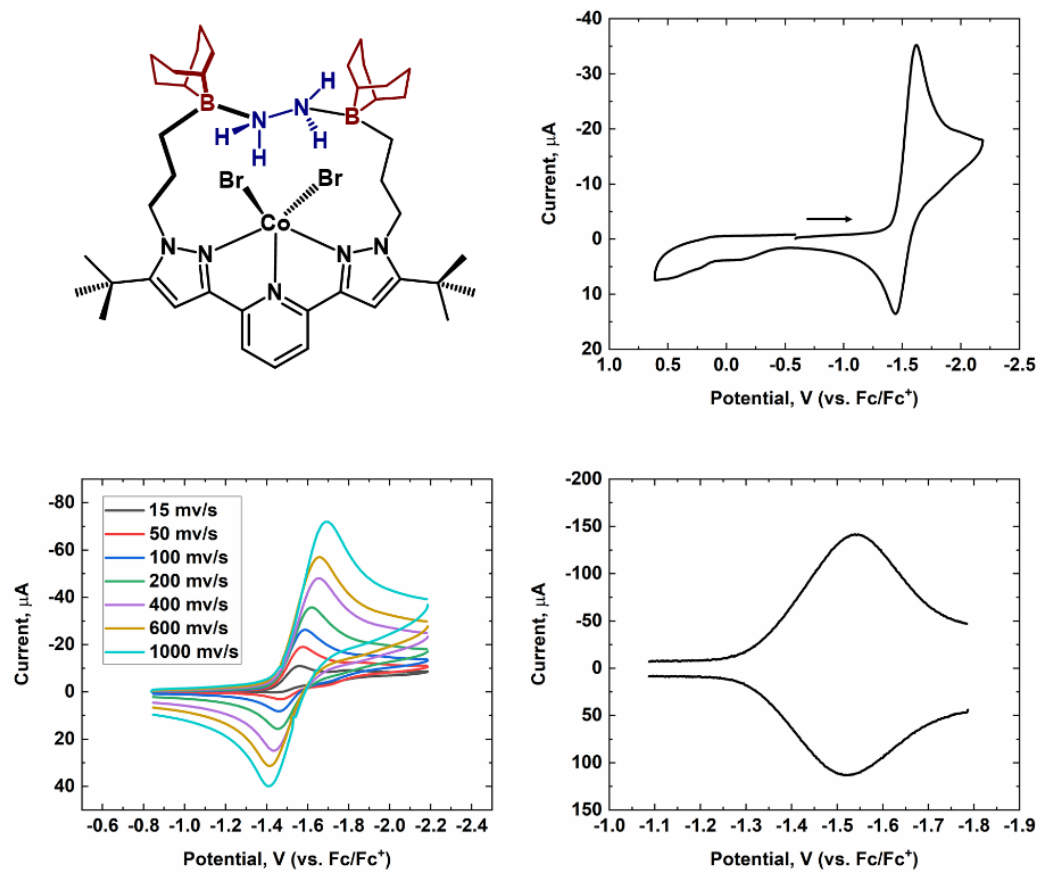

Figure S74 Electrochemical data for ( $\left.{ }^{\mathrm{BBN} P D P}{ }^{t B u}\right) \mathrm{CoBr}_{2}\left(\mathrm{~N}_{2} \mathrm{H}_{4}\right)(0.7 \mathrm{mM})$ recorded in THF with $0.2 \mathrm{M}$ $\left[\mathrm{Bu}_{4} \mathrm{~N}\right]\left[\mathrm{PF}_{6}\right]$. Working electrode: glassy carbon; counter electrode: platinum; reference electrode: silver wire. Top: full scan. Bottom: square wave voltammogram and scan rate dependence. 

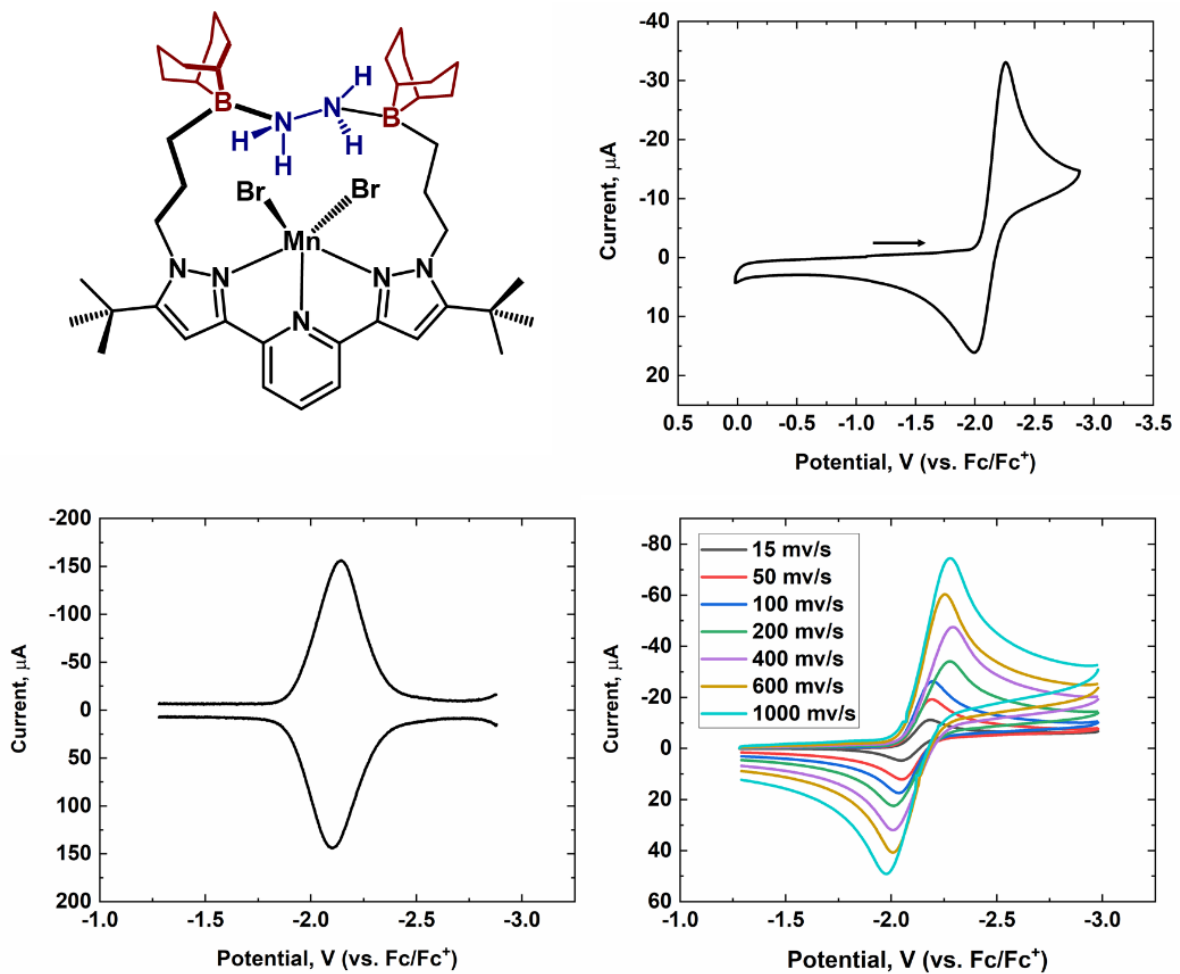

Figure S75 Electrochemical data for ( $\left.{ }^{\mathrm{BBN}} \mathrm{PDP}{ }^{\mathrm{tBu}}\right) \mathrm{MnBr}_{2}\left(\mathrm{~N}_{2} \mathrm{H}_{4}\right)(0.7 \mathrm{mM})$ recorded in THF with $0.2 \mathrm{M}$ $\left[\mathrm{Bu}_{4} \mathrm{~N}\right]\left[\mathrm{PF}_{6}\right]$. Working electrode: glassy carbon; counter electrode: platinum; reference electrode: silver wire. Top: full scan. Bottom: square wave voltammogram and scan rate dependence.
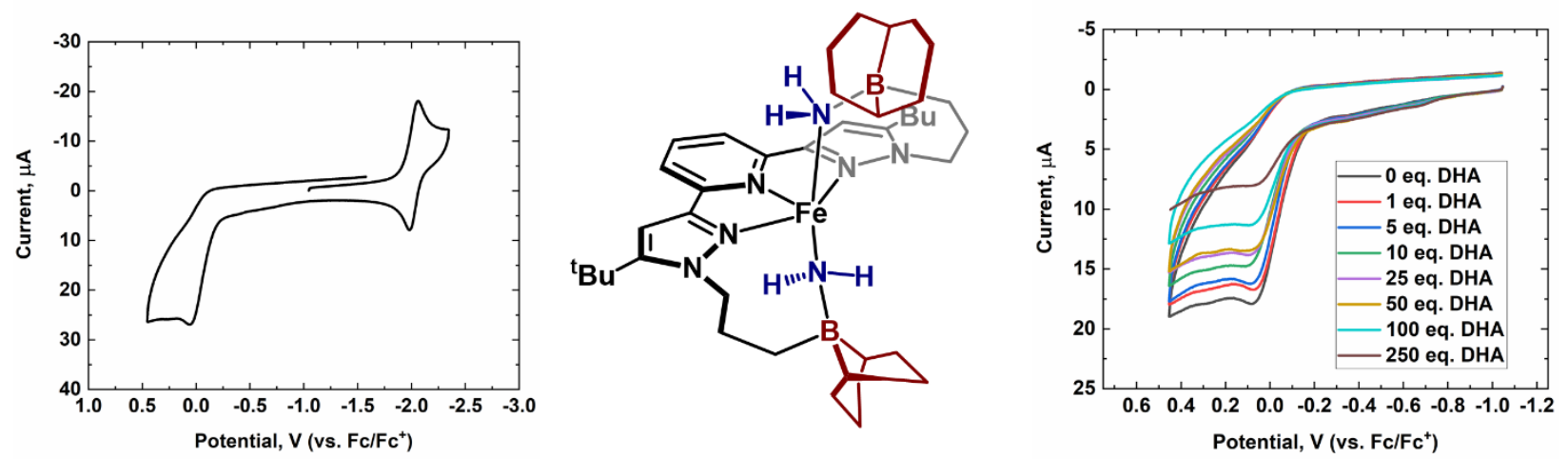

Figure S76 Electrochemical data for $\left({ }^{\mathrm{BBN} P D P}{ }^{\mathrm{BB}}\right) \mathrm{Fe}\left(\mathrm{NH}_{2}\right)_{2}(1.0 \mathrm{mM})$ recorded in DMF with $0.1 \mathrm{M}$ $\left[\mathrm{Bu}_{4} \mathrm{~N}\right]\left[\mathrm{PF}_{6}\right]$. Working electrode: glassy carbon; counter electrode: platinum; reference electrode: $\mathrm{Ag} / \mathrm{AgNO}_{3}$. Left: full scan. Right: dependence of 9,10-dihydroanthracene on oxidative wave. 

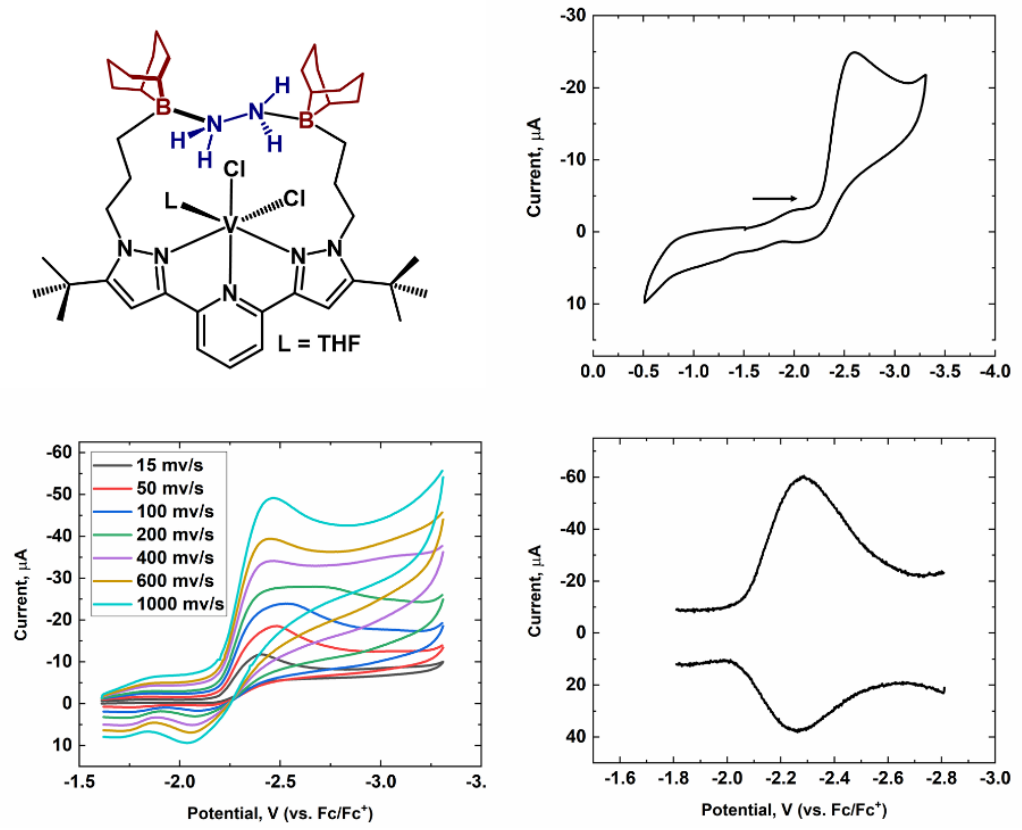

Figure S77 Electrochemical data for ( $\left.{ }^{\mathrm{BBN} P D P}{ }^{\mathrm{tBu}}\right) \mathrm{VCl}_{2}\left(\mathrm{~N}_{2} \mathrm{H}_{4}\right)(\mathrm{THF})(0.8 \mathrm{mM})$ recorded in THF with $0.2 \mathrm{M}$ $\left[\mathrm{Bu}_{4} \mathrm{~N}\right]\left[\mathrm{PF}_{6}\right]$. Working electrode: glassy carbon; counter electrode: platinum; reference electrode: silver wire. Top: full scan. Bottom: scan rate dependence and square wave voltammogram.
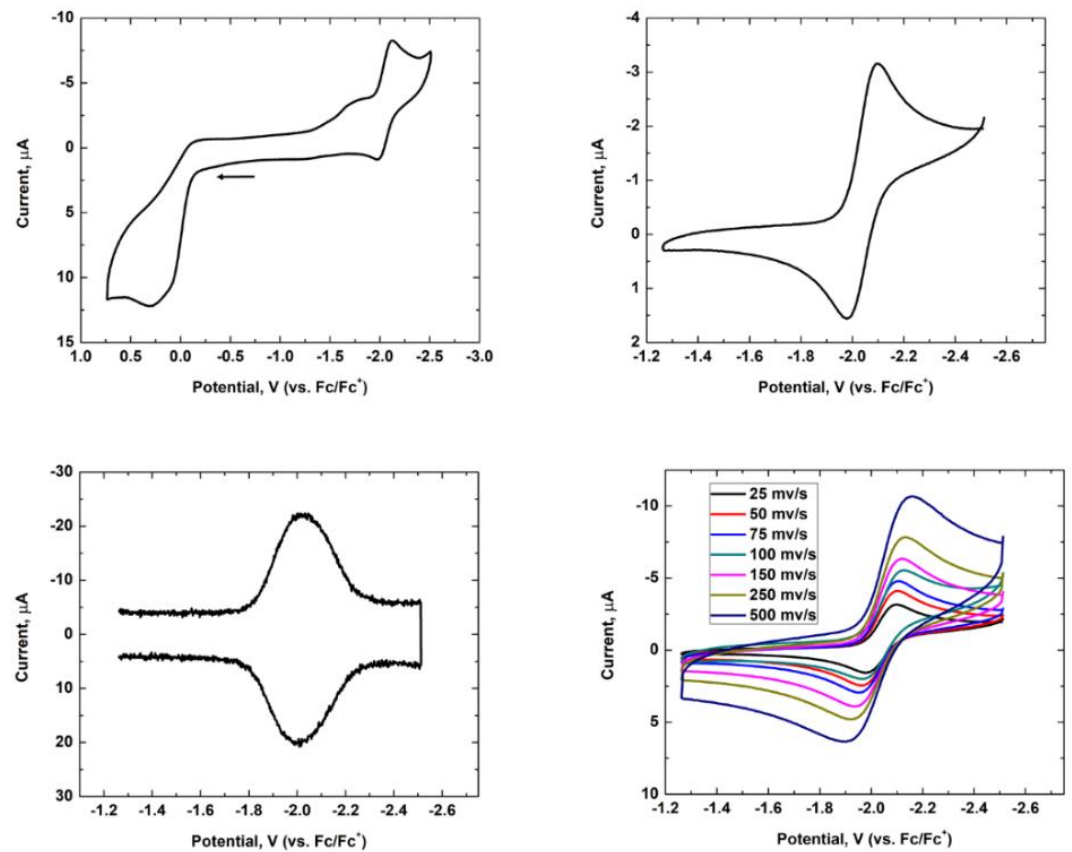

Figure S78 Electrochemical data for $\left({ }^{\mathrm{BBN} P D P}{ }^{\mathrm{BBu}}\right) \mathrm{Co}\left(\mathrm{NH}_{2}\right)_{2}(0.5 \mathrm{mM})$ recorded in THF with $0.2 \mathrm{M}$ $\left[\mathrm{Bu}_{4} \mathrm{~N}\right]\left[\mathrm{PF}_{6}\right]$. Working electrode: glassy carbon; counter electrode: platinum; reference electrode: silver wire. Top: full scan and isolated reduction wave. Bottom: square wave voltammogram and scan rate dependence. 

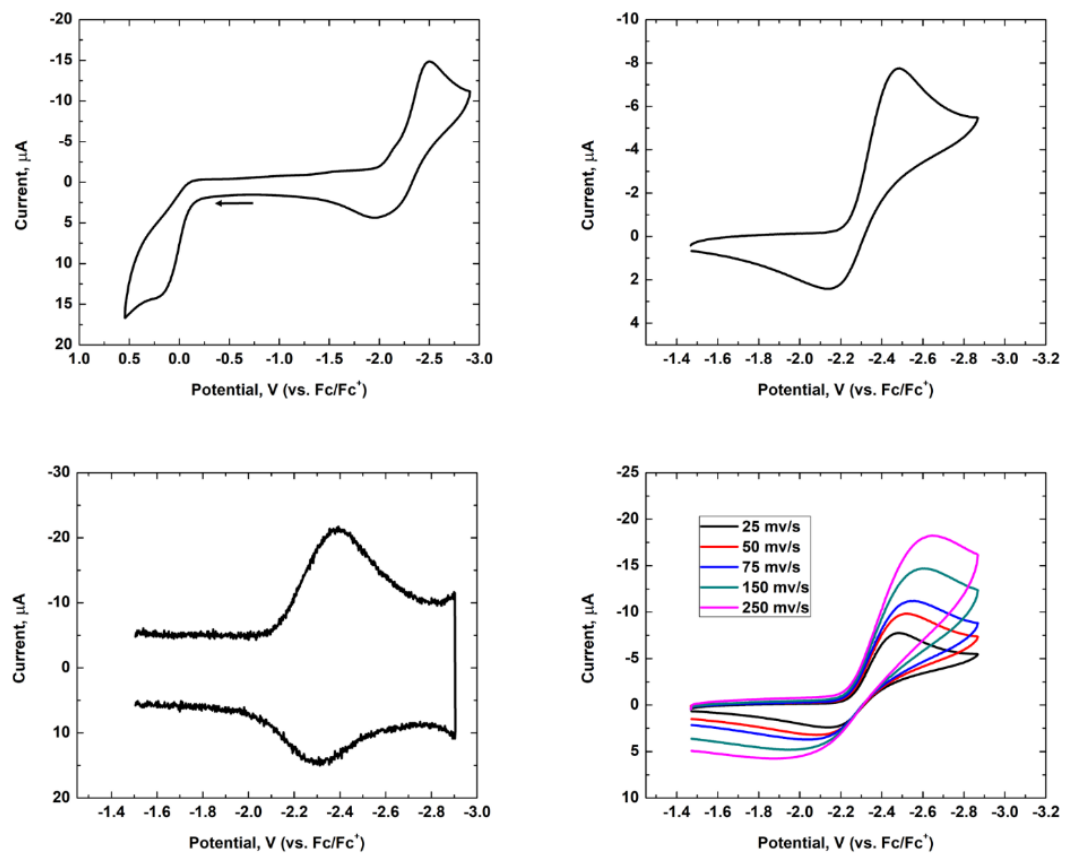

Figure S79 Electrochemical data for $\left({ }^{\mathrm{BBN} P D P}{ }^{\mathrm{BBu}}\right) \mathrm{Mn}\left(\mathrm{NH}_{2}\right)_{2}(1.0 \mathrm{mM})$ recorded in THF with $0.2 \mathrm{M}$ $\left[\mathrm{Bu}_{4} \mathrm{~N}\right]\left[\mathrm{PF}_{6}\right]$. Working electrode: glassy carbon; counter electrode: platinum; reference electrode: silver wire. Top: full scan and isolated reduction wave. Bottom: square wave voltammogram and scan rate dependence. 

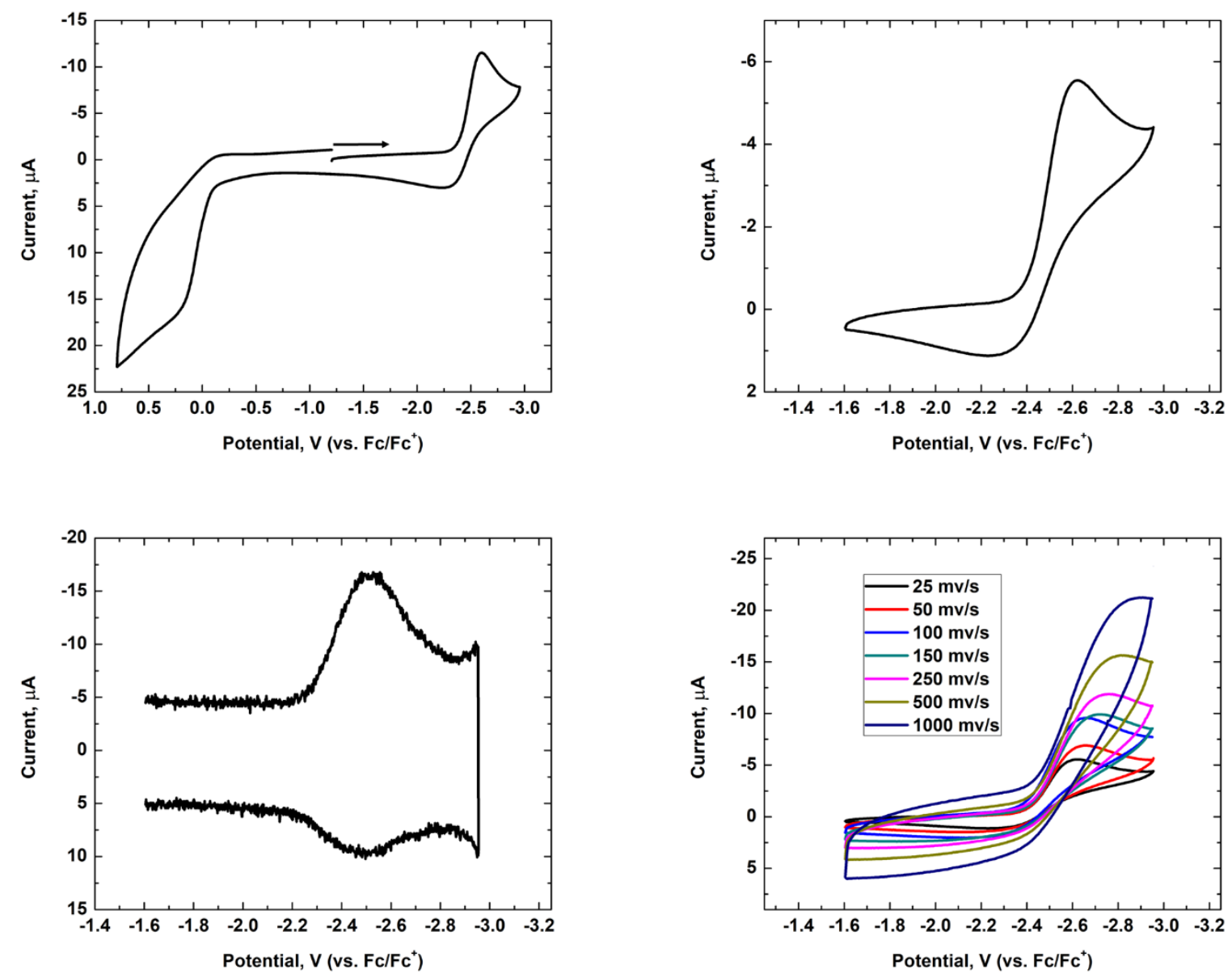

Figure $\mathbf{S 8 0}$ Electrochemical data for $\left({ }^{\mathrm{BBN}} \mathrm{PDP}{ }^{\mathrm{tBu}}\right) \mathrm{Zn}\left(\mathrm{NH}_{2}\right)_{2}(0.5 \mathrm{mM})$ recorded in THF with $0.2 \mathrm{M}$ $\left[\mathrm{Bu}_{4} \mathrm{~N}\right]\left[\mathrm{PF}_{6}\right]$. Working electrode: glassy carbon; counter electrode: platinum; reference electrode: silver wire. Top: full scan and isolated reduction wave. Bottom: square wave voltammogram and scan rate dependence.
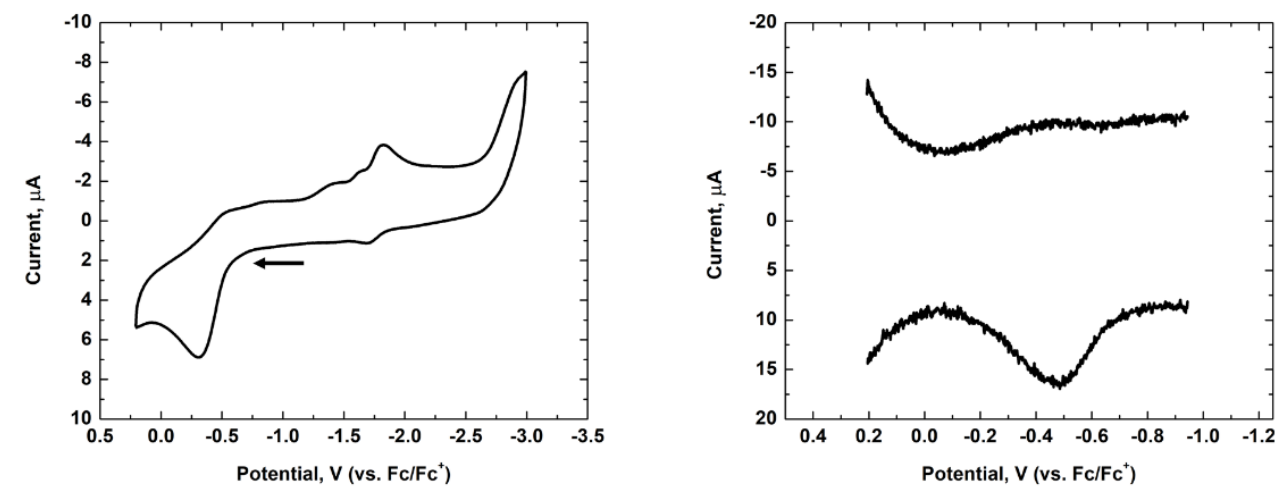

Figure S81 Electrochemical data for [K(2,2,2-crypt)][(benzyl-BBNPDP $\left.\left.{ }^{\mathrm{BBu}}\right) \mathrm{Fe}\left(\mathrm{NH}_{2}\right)_{2}\right](0.5 \mathrm{mM})$ recorded in THF with $0.2 \mathrm{M}\left[\mathrm{Bu}_{4} \mathrm{~N}\right]\left[\mathrm{PF}_{6}\right]$. Working electrode: glassy carbon; counter electrode: platinum; reference electrode: silver wire. Left: full scan; right: square wave voltammogram. 

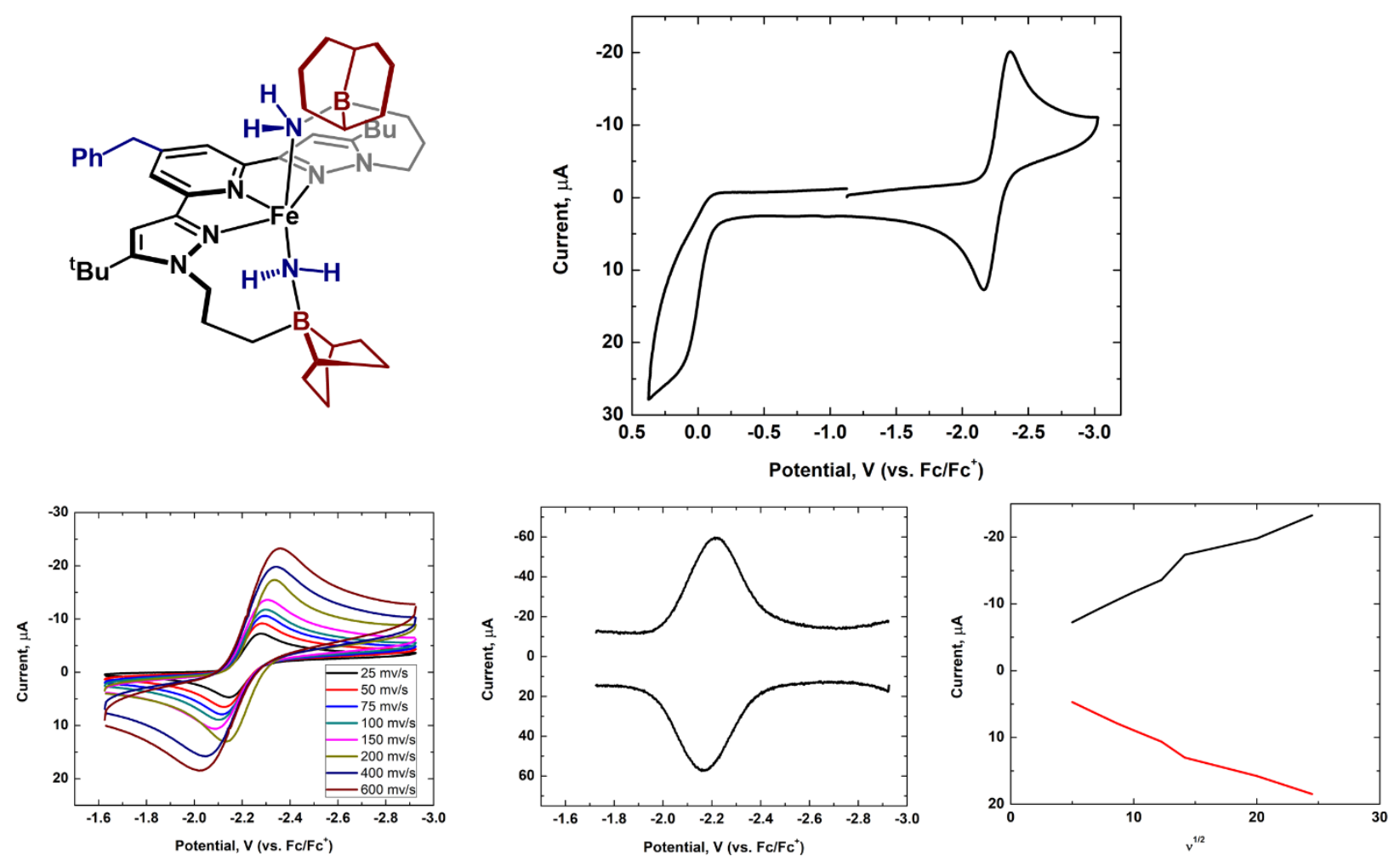

Figure S82 Electrochemical data for (benzyl- $\left.{ }^{B B N} P D P{ }^{t B u}\right) F e\left(N_{2}\right)_{2}(0.8 \mathrm{mM})$ recorded in THF with $0.2 \mathrm{M}$ $\left[\mathrm{Bu}_{4} \mathrm{~N}\right]\left[\mathrm{PF}_{6}\right]$. Working electrode: glassy carbon; counter electrode: platinum; reference electrode: silver wire. Top: full scan. Bottom: scan rate dependence of reductive wave, square wave voltammogram, and plot of $v^{1 / 2}$ vs current.

Table S2 Electrochemical properties assessed by square wave voltammetry (vs. $\mathrm{Fc} / \mathrm{Fc}^{+}$).

\begin{tabular}{|l|c|c|c|c|c|}
\hline & $\mathbf{1 - V}$ & $\mathbf{1 - M n}$ & $\mathbf{1 - \mathbf { F e } ^ { \mathrm { a } }}$ & $\mathbf{1 - C o}$ & $\mathbf{1 - \mathbf { Z n } ^ { \mathrm { a } }}$ \\
\hline $\mathbf{E}_{\text {oxidation }}(\mathbf{V})$ & -- & 0.52 & 0.09 & 0.58 & 0.92 \\
\hline $\mathbf{E}_{\text {reduction }}(\mathbf{V})$ & $-1.45 /-2.25$ & -2.20 & -2.14 & -1.85 & -2.36 \\
\hline
\end{tabular}

a Data from J. Am. Chem. Soc. 2017, 139, 18194-18197 for comparison

Table S3 Electrochemical properties assessed by square wave voltammetry (vs. Fc/Fc ${ }^{+}$).

\begin{tabular}{|l|c|c|c|c|c|}
\hline & $\mathbf{2 - V}$ & $\mathbf{2 - M n}$ & $\mathbf{2 - F e}$ & $\mathbf{2 - C o}$ & $\mathbf{2 - Z n ^ { \mathbf { a } }}$ \\
\hline$E_{\text {reduction }}$ (V) & -2.28 & -2.12 & -1.96 & -1.53 & -2.04 \\
\hline
\end{tabular}

${ }^{a}$ Data from J. Am. Chem. Soc. 2017, 139, 18194-18197 for comparison

Table S4 Electrochemical properties assessed by square wave voltammetry (vs. $\mathrm{Fc} / \mathrm{Fc}^{+}$).

\begin{tabular}{|l|c|c|c|c|c|c|}
\hline & 4-Mn & 4-Fe $^{\mathbf{a}}$ & 4-Co & 4-Zn & $\mathbf{6}$ & $\mathbf{7}$ \\
\hline $\mathbf{E}_{\text {oxidation }}$ (V) & 0.09 & $0.16 /-0.02$ & 0.04 & 0.11 & -0.49 & -- \\
\hline $\mathbf{E}_{\text {reduction }}$ (V) & -2.32 & -2.12 & -2.02 & -2.50 & -- & -2.19 \\
\hline
\end{tabular}

${ }^{a}$ Data from J. Am. Chem. Soc. 2017, 139, 18194-18197 for comparison 
Complex: $\left({ }^{\mathrm{BBN}} \mathrm{PDP}{ }^{\mathrm{BBu}}\right) \mathrm{VCl}_{3}$

Local name: jk1222

CCDC 1993628

Table S5 Crystallographic experimental parameters for $\left({ }^{\mathrm{BBN}} \mathrm{PDP}^{\mathrm{tBu}}\right) \mathrm{VCl}_{3}$.

\begin{tabular}{|c|c|}
\hline \multicolumn{2}{|l|}{ Crystal data } \\
\hline Chemical formula & $\mathrm{C}_{41} \mathrm{H}_{63} \mathrm{~B}_{2} \mathrm{Cl}_{3} \mathrm{~N}_{5} \mathrm{~V}$ \\
\hline Mr & 804.87 \\
\hline Crystal system, space group & Monoclinic, $P 2_{1} / c$ \\
\hline Temperature (K) & 150 \\
\hline$a, b, c(\AA)$ & 18.0437 (5), $23.0342(6), 10.3990$ (3) \\
\hline$\beta\left(^{\circ}\right)$ & $100.1786(13)$ \\
\hline$V\left(\AA^{3}\right)$ & $4254.0(2)$ \\
\hline$Z$ & 4 \\
\hline Radiation type & $\mathrm{Cu} K \alpha$ \\
\hline$\mu\left(\mathrm{mm}^{-1}\right)$ & 3.94 \\
\hline Crystal size $(\mathrm{mm})$ & $0.32 \times 0.07 \times 0.05$ \\
\hline \multicolumn{2}{|l|}{ Data collection } \\
\hline Diffractometer & Bruker AXS D8 Quest CMOS diffractometer \\
\hline Absorption correction & $\begin{array}{l}\text { Multi-scan SADABS 2016/2: Krause, L., Herbst-Irmer, R., Sheldrick } \\
\text { G.M. \& Stalke D., J. Appl. Cryst. } 48 \text { (2015) 3-10 }\end{array}$ \\
\hline$T_{\min }, T_{\max }$ & $0.591,0.754$ \\
\hline $\begin{array}{l}\text { No. of measured, independent and } \\
\text { observed }[I>2 s(I)] \text { reflections }\end{array}$ & $41146,9000,7330$ \\
\hline$R_{\text {int }}$ & 0.063 \\
\hline$(\sin \theta / \lambda)_{\max }\left(\AA^{-1}\right)$ & 0.639 \\
\hline \multicolumn{2}{|l|}{ Refinement } \\
\hline$R\left[F^{2}>2 \sigma\left(F^{2}\right)\right], w R\left(F^{2}\right), S$ & $0.057,0.158,1.05$ \\
\hline No. of reflections & 9000 \\
\hline No. of parameters & 676 \\
\hline No. of restraints & 969 \\
\hline $\mathrm{H}$-atom treatment & $\mathrm{H}$-atom parameters constrained \\
\hline$\Delta \rho_{\max }, \Delta \rho_{\min }\left(\mathrm{e} \AA^{-3}\right)$ & $0.62,-0.47$ \\
\hline
\end{tabular}

Computer programs: Apex3 v2016.9-0 (Bruker, 2016), SAINT V8.37A (Bruker, 2016), SHELXS97

(Sheldrick, 2008), SHELXL2016/6 (Sheldrick, 2015, 2016), SHELXLE Rev714 (Hübschle et al., 2011). 


\section{Refinement details:}

One of the 9-BBN units is disordered. It was refined as distributed over three different orientations. Their geometries were restrained to be similar to that of the not disordered equivalent unit, and $\mathrm{U}^{\mathrm{ij}}$ components of ADPs were restrained to be similar for disordered atoms closer to each other than $2.0 \AA$. A mild anti-bumping restraint was applied to keep minor moiety $\mathrm{H}$ atoms from approaching other $\mathrm{H}$ atoms too closely. Subject to these conditions the occupancy rates refined to $0.659(3), 0.180(3)$, and $0.161(2)$.

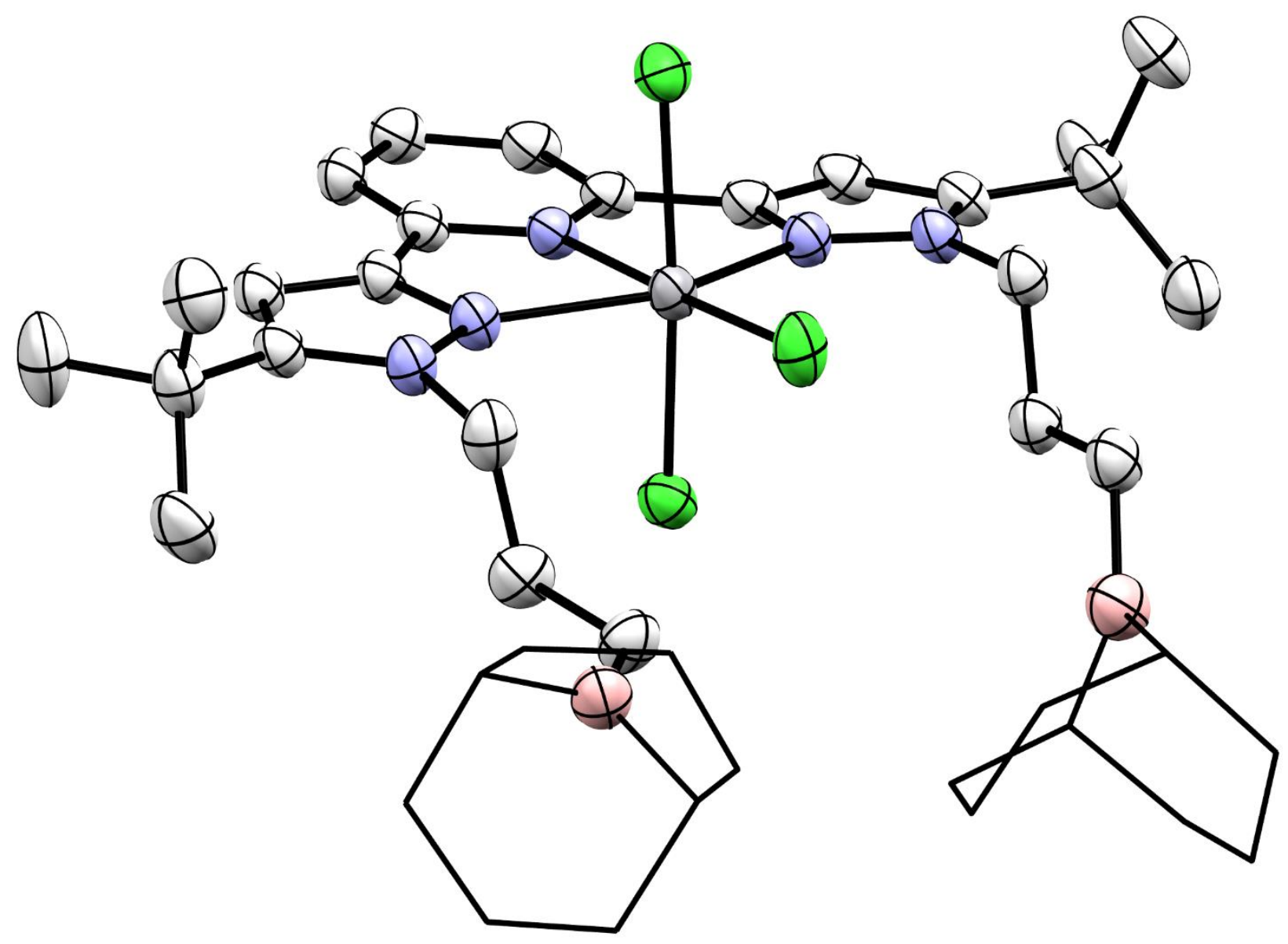

Figure S83 Molecular structure of ( $\left.{ }^{\mathrm{BBN}} \mathrm{PDP}^{\mathrm{tBu}}\right) \mathrm{VCl}_{3}$ displayed with $50 \%$ probability ellipsoids. Hydrogen atoms not attached to heteroatoms and disorder are omitted for clarity. The 9-BBN substituents are displayed in wireframe for clarity. 
Complex: ( $\left.{ }^{\mathrm{BBN} P D P}{ }^{t \mathrm{Bu}}\right) \mathrm{CoBr}_{2}\left(\mathrm{~N}_{2} \mathrm{H}_{4}\right)$

Local name: jk5231

CCDC 1993626

Table S6 Crystallographic experimental parameters for ( $\left.{ }^{\mathrm{BBN} P D P}{ }^{\mathrm{BBu}}\right) \mathrm{CoBr}_{2}\left(\mathrm{~N}_{2} \mathrm{H}_{4}\right)$.

\begin{tabular}{|c|c|}
\hline \multicolumn{2}{|l|}{ Crystal data } \\
\hline Chemical formula & $\mathrm{C}_{41} \mathrm{H}_{67} \mathrm{~B}_{2} \mathrm{Br}_{2} \mathrm{CoN}_{7} \cdot \mathrm{CH}_{2} \mathrm{Cl}_{2}$ \\
\hline$M r$ & 983.31 \\
\hline Crystal system, space group & Monoclinic, $P 2_{1} / c$ \\
\hline Temperature (K) & 150 \\
\hline$a, b, c(\AA)$ & 17.5351 (3), $13.0586(3), 21.0879$ (4) \\
\hline$\beta\left(^{\circ}\right)$ & $108.2710(8)$ \\
\hline$V\left(\AA^{3}\right)$ & $4585.34(16)$ \\
\hline$Z$ & 4 \\
\hline Radiation type & $\mathrm{Cu} K \alpha$ \\
\hline$\mu\left(\mathrm{mm}^{-1}\right)$ & 6.33 \\
\hline Crystal size $(\mathrm{mm})$ & $0.22 \times 0.13 \times 0.08$ \\
\hline \multicolumn{2}{|l|}{ Data collection } \\
\hline Diffractometer & $\begin{array}{l}\text { Bruker AXS D8 Quest CMOS diffractometer with Photonll charge- } \\
\text { integrating pixel array detector (CPAD) }\end{array}$ \\
\hline Absorption correction & $\begin{array}{l}\text { Multi-scan SADABS 2016/2: Krause, L., Herbst-Irmer, R., Sheldrick G.M } \\
\text { \& Stalke D., J. Appl. Cryst. } 48 \text { (2015) 3-10 }\end{array}$ \\
\hline$T_{\min }, T_{\max }$ & $0.334,0.754$ \\
\hline $\begin{array}{l}\text { No. of measured, independent and } \\
\text { observed }[I>2 \mathrm{~s}(I)] \text { reflections }\end{array}$ & $33730,9573,8036$ \\
\hline$R_{\text {int }}$ & 0.046 \\
\hline$(\sin \theta / \lambda)_{\max }\left(\AA^{-1}\right)$ & 0.640 \\
\hline \multicolumn{2}{|l|}{ Refinement } \\
\hline$R\left[F^{2}>2 \sigma\left(F^{2}\right)\right], w R\left(F^{2}\right), S$ & $0.046,0.132,1.09$ \\
\hline No. of reflections & 9573 \\
\hline No. of parameters & 567 \\
\hline No. of restraints & 140 \\
\hline $\mathrm{H}$-atom treatment & $\mathrm{H}$-atom parameters constrained \\
\hline$\Delta \rho_{\max }, \Delta \rho_{\min }\left(\mathrm{e} \AA^{-3}\right)$ & $2.13,-1.35$ \\
\hline
\end{tabular}

Computer programs: Apex3 v2018.1-0 (Bruker, 2018), SAINT V8.38A (Bruker, 2016), SHELXS97

(Sheldrick, 2008), SHELXL2018/3 (Sheldrick, 2015, 2018), SHELXLE Rev1030 (Hübschle et al., 2011). 


\section{Refinement details:}

One of the propylene chains and a solvate methylene chloride molecule were refined as disordered. The major and minor moieties were each restrained to have similar geometries. $\mathrm{U}^{\mathrm{ij}}$ components of ADPs for disordered atoms closer to each other than $2.0 \AA \AA$ were restrained to be similar. Subject to these conditions the occupancy ratio refined to $0.876(6)$ to $0.124(6)$ for the propylene chain, and to $0.807(8)$ to $0.193(8)$ for the solvate molecule.

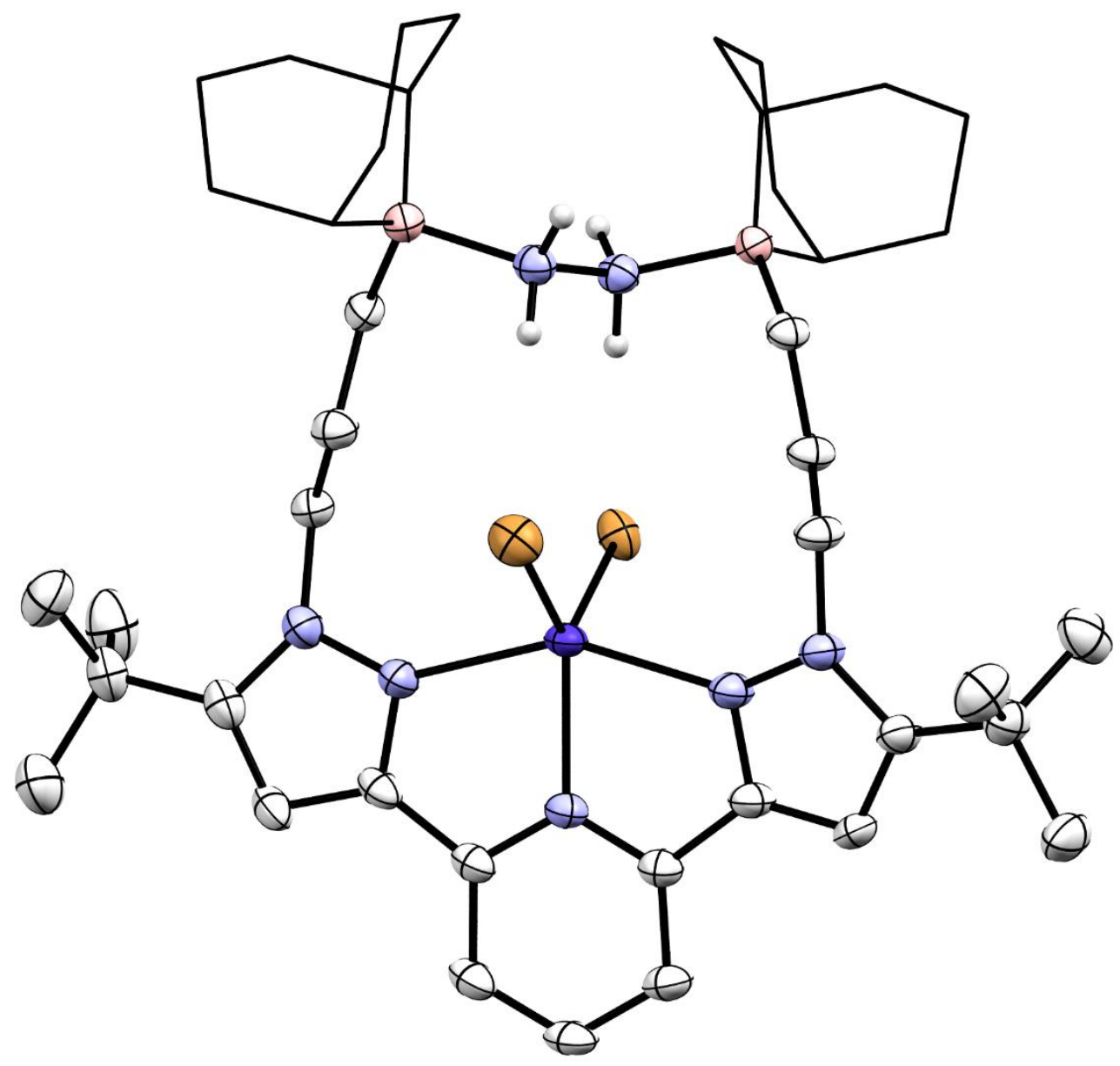

Figure S84 Molecular structure of ( $\left.{ }^{\mathrm{BBN} P D P}{ }^{t \mathrm{Bu}}\right) \mathrm{CoBr}_{2}\left(\mathrm{~N}_{2} \mathrm{H}_{4}\right)$ displayed with $50 \%$ probability ellipsoids. Hydrogen atoms not attached to heteroatoms, disorder and solvate molecules are omitted for clarity. The 9-BBN substituents are displayed in wireframe for clarity. 
Complex: ( $\left.{ }^{\mathrm{BBN} P D P^{t B u}}\right) \mathrm{MnBr}_{2}\left(\mathrm{~N}_{2} \mathrm{H}_{4}\right)$

Local name: jk5240

CCDC 1993625

Table S7 Crystallographic experimental parameters for ( $\left.{ }^{\mathrm{BBN} P D P}{ }^{\mathrm{BBu}}\right) \mathrm{MnBr}_{2}\left(\mathrm{~N}_{2} \mathrm{H}_{4}\right)$.

\begin{tabular}{|c|c|}
\hline \multicolumn{2}{|l|}{ Crystal data } \\
\hline Chemical formula & $\mathrm{C}_{41} \mathrm{H}_{67} \mathrm{~B}_{2} \mathrm{Br}_{2} \mathrm{MnN}_{7} \cdot \mathrm{CH}_{2} \mathrm{Cl}_{2}$ \\
\hline$M r$ & 979.32 \\
\hline Crystal system, space group & Monoclinic, $P 2{ }_{1} / c$ \\
\hline Temperature (K) & 150 \\
\hline$a, b, c(\AA)$ & $17.4574(10), 13.1941(8), 21.1151$ (12) \\
\hline$\beta\left({ }^{\circ}\right)$ & $108.006(2)$ \\
\hline$V\left(\AA^{3}\right)$ & $4625.4(5)$ \\
\hline$Z$ & 4 \\
\hline Radiation type & $\mathrm{Cu} K \alpha$ \\
\hline$\mu\left(\mathrm{mm}^{-1}\right)$ & 5.69 \\
\hline Crystal size (mm) & $0.13 \times 0.11 \times 0.01$ \\
\hline \multicolumn{2}{|l|}{ Data collection } \\
\hline Diffractometer & $\begin{array}{l}\text { Bruker AXS D8 Quest CMOS diffractometer with Photonll charge- } \\
\text { integrating pixel array detector (CPAD) }\end{array}$ \\
\hline Absorption correction & $\begin{array}{l}\text { Multi-scan TWINABS 2012/1: Krause, L., Herbst-Irmer, R., Sheldrick } \\
\text { G.M. \& Stalke D. (2015). J. Appl. Cryst. } 48 \text { 3-10. }\end{array}$ \\
\hline$T_{\min }, T_{\max }$ & $0.496,0.754$ \\
\hline $\begin{array}{l}\text { No. of measured, independent and } \\
\text { observed }[I>2 s(I)] \text { reflections }\end{array}$ & $60172,9682,8003$ \\
\hline$R_{\text {int }}$ & 0.073 \\
\hline$(\sin \theta / \lambda)_{\max }\left(\AA^{-1}\right)$ & 0.641 \\
\hline \multicolumn{2}{|l|}{ Refinement } \\
\hline$R\left[F^{2}>2 \sigma\left(F^{2}\right)\right], w R\left(F^{2}\right), S$ & $0.049,0.132,1.07$ \\
\hline No. of reflections & 9682 \\
\hline No. of parameters & 511 \\
\hline $\mathrm{H}$-atom treatment & $\mathrm{H}$-atom parameters constrained \\
\hline$\Delta \rho_{\max }, \Delta \rho_{\min }\left(\mathrm{e} \AA^{-3}\right)$ & $1.09,-1.02$ \\
\hline
\end{tabular}

Computer programs: Apex3 v2018.7-2 (Bruker, 2018), SAINT V8.38A (Bruker, 2018), SHELXS97

(Sheldrick, 2008), SHELXL2018/3 (Sheldrick, 2015, 2018), SHELXLE Rev1030 (Hübschle et al., 2011). 


\section{Refinement details:}

Crystals were small and heavily intergrown in clusters of platelets. The least intergrown and still sufficiently sized crystal that could be isolated was found to be two-component.

The orientation matrices for the two components were identified using the program Cell_Now, with the two components being related by no obvious twin operation (a 5.4 degree rotation around real axis 0.0061 .000 0.043). The two components were integrated using Saint and corrected for absorption using twinabs, resulting in the following statistics:

27892 data (8483 unique) involve domain 1 only, mean I/sigma 10.7

27823 data (8491 unique) involve domain 2 only, mean I/sigma 7.7

4535 data (2181 unique) involve 2 domains, mean I/sigma 13.4

The exact transformation matrix identified by the integration program was found to be:

$0.964410 .00678-0.08309$

$-0.003760 .999990 .00027$

$0.12144-0.002381 .02643$

The structure was solved using direct methods with only the non-overlapping reflections of both components. The structure was refined against the same data (i.e., not as a twin but using the HKLF 4 routine).

The $R_{\text {int }}$ value given is for all reflections and is based on agreement between observed single and composite intensities and those calculated from refined unique intensities and twin fractions (TWINABS (Sheldrick, 2012)).

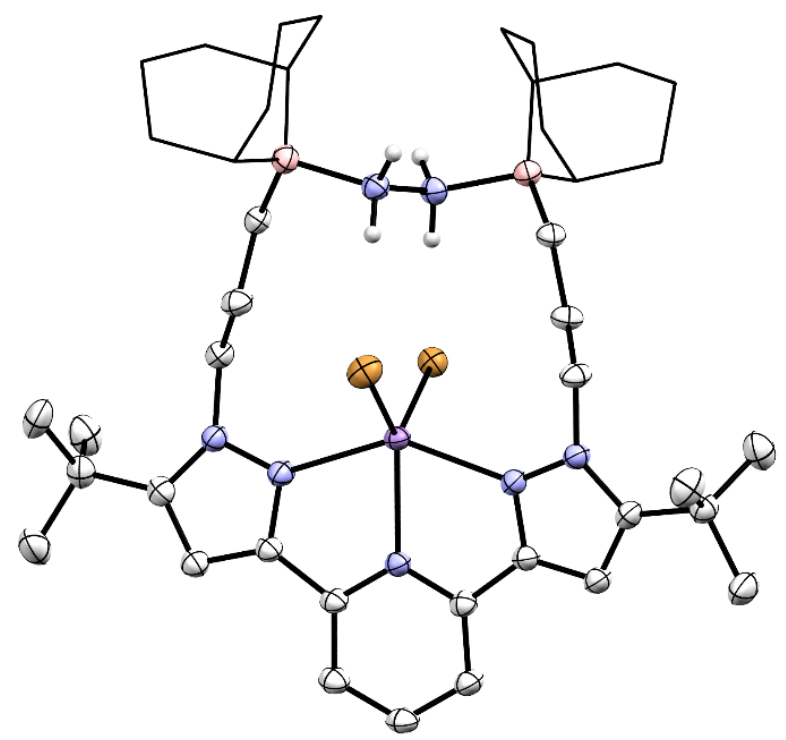

Figure S85 Molecular structure of ( $\left.{ }^{\mathrm{BBN} P D P}{ }^{t B u}\right) \mathrm{MnBr}_{2}\left(\mathrm{~N}_{2} \mathrm{H}_{4}\right)$ displayed with 50\% probability ellipsoids. Hydrogen atoms not attached to heteroatoms and solvate molecules are omitted for clarity. The 9-BBN substituents are displayed in wireframe for clarity. 
Complex: ( $\left.{ }^{B B N P D P t B u}\right) V_{C l}\left(\mathrm{~N}_{2} \mathrm{H}_{4}\right)(\mathrm{THF})$

Local name: jk5233

CCDC 1993629

Table S8 Crystallographic experimental parameters for ( ${ }^{\left.\mathrm{BBN} P D P^{\mathrm{Bu}}\right)} \mathrm{VCl}_{2}\left(\mathrm{~N}_{2} \mathrm{H}_{4}\right)(\mathrm{THF})$.

\begin{tabular}{|c|c|}
\hline \multicolumn{2}{|l|}{ Crystal data } \\
\hline Chemical formula & $\mathrm{C}_{45} \mathrm{H}_{75} \mathrm{~B}_{2} \mathrm{Cl}_{2} \mathrm{~N}_{7} \mathrm{OV} \cdot \mathrm{C}_{4} \mathrm{H}_{8} \mathrm{O}$ \\
\hline$M r$ & 945.68 \\
\hline Crystal system, space group & Triclinic, $P \overline{1}$ \\
\hline Temperature (K) & 150 \\
\hline$a, b, c(\AA)$ & $12.1569(8), 13.9602(10), 16.4539$ (12) \\
\hline$\alpha, \beta, \gamma\left({ }^{\circ}\right)$ & $67.728(2), 82.212(3), 89.849$ (3) \\
\hline$V\left(\AA^{3}\right)$ & 2556.7 (3) \\
\hline$Z$ & 2 \\
\hline Radiation type & 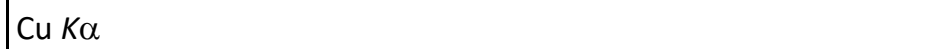 \\
\hline$\mu\left(\mathrm{mm}^{-1}\right)$ & 2.92 \\
\hline Crystal size (mm) & $0.23 \times 0.15 \times 0.14$ \\
\hline \multicolumn{2}{|l|}{ Data collection } \\
\hline Diffractometer & $\begin{array}{l}\text { Bruker AXS D8 Quest CMOS diffractometer with Photonll charge- } \\
\text { integrating pixel array detector (CPAD) }\end{array}$ \\
\hline Absorption correction & $\begin{array}{l}\text { Multi-scan SADABS 2016/2: Krause, L., Herbst-Irmer, R., Sheldrick } \\
\text { G.M. \& Stalke D., J. Appl. Cryst. } 48 \text { (2015) 3-10 }\end{array}$ \\
\hline$T_{\min }, T_{\max }$ & $0.564,0.754$ \\
\hline $\begin{array}{l}\text { No. of measured, independent and } \\
\text { observed }[I>2 s(I)] \text { reflections }\end{array}$ & $29016,10315,9472$ \\
\hline$R_{\text {int }}$ & 0.035 \\
\hline$(\sin \theta / \lambda)_{\max }\left(\AA^{-1}\right)$ & 0.640 \\
\hline \multicolumn{2}{|l|}{ Refinement } \\
\hline$R\left[F^{2}>2 \sigma\left(F^{2}\right)\right], w R\left(F^{2}\right), S$ & $0.041,0.112,1.11$ \\
\hline No. of reflections & 10315 \\
\hline No. of parameters & 574 \\
\hline $\mathrm{H}$-atom treatment & $\mathrm{H}$-atom parameters constrained \\
\hline$\Delta \rho_{\max }, \Delta \rho_{\min }\left(\mathrm{e} \AA^{-3}\right)$ & $0.36,-0.37$ \\
\hline
\end{tabular}

Computer programs: Apex3 v2018.7-2 (Bruker, 2018), SAINT V8.38A (Bruker, 2018), SHELXS97

(Sheldrick, 2008), SHELXL2018/3 (Sheldrick, 2015, 2018), SHELXLE Rev1030 (Hübschle et al., 2011). 


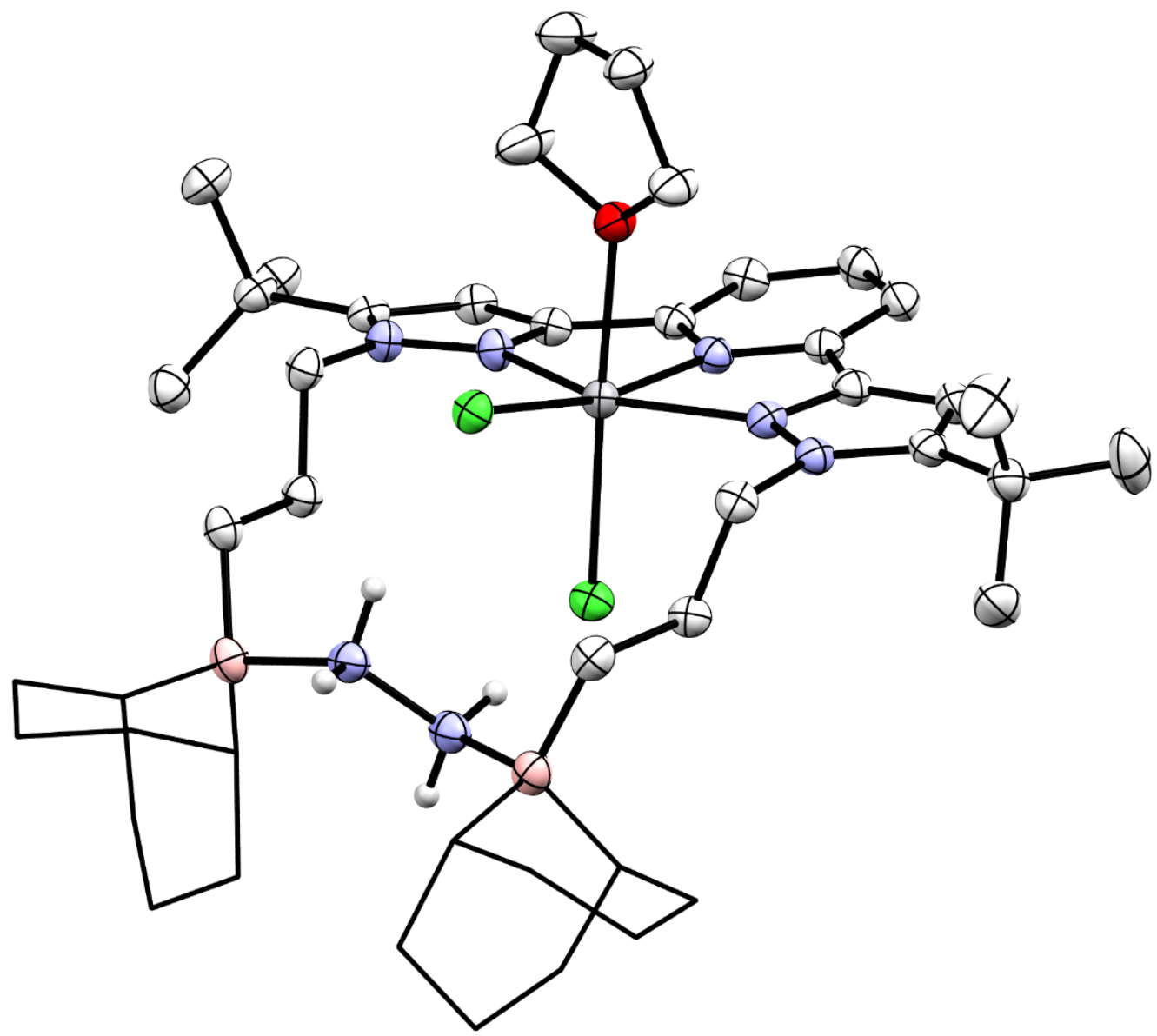

Figure S86 Molecular structure of ( $\left.{ }^{B B N P D P}{ }^{\mathrm{BBu}}\right) \mathrm{VCl}_{2}\left(\mathrm{~N}_{2} \mathrm{H}_{4}\right)(\mathrm{THF})$ displayed with $50 \%$ probability ellipsoids. Hydrogen atoms not attached to heteroatoms and solvate molecules are omitted for clarity. The 9-BBN substituents are displayed in wireframe for clarity. 
Complex: ( $\left.{ }^{B B N} \mathrm{PDP}{ }^{\mathrm{BBu}}\right) \mathrm{MnBr}_{2}\left(\mathrm{~N}_{2} \mathrm{H}_{4}\right)_{2}$

Local name: jk5232

CCDC 1993633

Table S9 Crystallographic experimental parameters for ( $\left.{ }^{\mathrm{BBN} P D P} \mathrm{P}^{t \mathrm{Bu}}\right) \mathrm{MnBr}_{2}\left(\mathrm{~N}_{2} \mathrm{H}_{4}\right)_{2}$.

\begin{tabular}{|c|c|}
\hline \multicolumn{2}{|l|}{ Crystal data } \\
\hline Chemical formula & $\mathrm{C}_{41} \mathrm{H}_{71} \mathrm{~B}_{2} \mathrm{Br}_{2} \mathrm{MnN}_{9} \cdot \mathrm{CH}_{2} \mathrm{Cl}_{2}$ \\
\hline$M r$ & 1011.37 \\
\hline Crystal system, space group & Triclinic, $P \overline{1}$ \\
\hline Temperature (K) & 150 \\
\hline$a, b, c(\AA)$ & $16.0123(15), 16.2797$ (19), 20.0984 (15) \\
\hline$\alpha, \beta, \gamma\left({ }^{\circ}\right)$ & $68.720(3), 78.005(5), 84.267(3)$ \\
\hline$V\left(\AA^{3}\right)$ & $4773.6(8)$ \\
\hline$Z$ & 4 \\
\hline Radiation type & Mo $K \alpha$ \\
\hline$\mu\left(\mathrm{mm}^{-1}\right)$ & 2.10 \\
\hline Crystal size (mm) & $0.25 \times 0.13 \times 0.04$ \\
\hline \multicolumn{2}{|l|}{ Data collection } \\
\hline Diffractometer & Bruker AXS D8 Quest CMOS diffractometer \\
\hline Absorption correction & $\begin{array}{l}\text { Multi-scan SADABS 2016/2: Krause, L., Herbst-Irmer, R., Sheldrick } \\
\text { G.M. \& Stalke D., J. Appl. Cryst. } 48 \text { (2015) 3-10 }\end{array}$ \\
\hline$T_{\min }, T_{\max }$ & $0.637,0.746$ \\
\hline $\begin{array}{l}\text { No. of measured, independent and } \\
\text { observed }[I>2 s(I)] \text { reflections }\end{array}$ & $58162,22240,12126$ \\
\hline$R$ int & 0.083 \\
\hline$(\sin \theta / \lambda)_{\max }\left(\AA^{-1}\right)$ & 0.668 \\
\hline \multicolumn{2}{|l|}{ Refinement } \\
\hline$R\left[F^{2}>2 \sigma\left(F^{2}\right)\right], w R\left(F^{2}\right), S$ & $0.068,0.171,1.02$ \\
\hline No. of reflections & 22240 \\
\hline No. of parameters & 1242 \\
\hline No. of restraints & 597 \\
\hline \multirow[t]{2}{*}{$\mathrm{H}$-atom treatment } & $\begin{array}{l}\mathrm{H} \text { atoms treated by a mixture of independent and constrained } \\
\text { refinement }\end{array}$ \\
\hline & $w=1 /\left[\mathrm{s}^{2}\left(F_{\mathrm{o}}^{2}\right)+(0.053 P)^{2}+12.9714 P\right]$ where $P=\left(F_{\mathrm{o}}^{2}+2 F_{\mathrm{c}}^{2}\right) / 3$ \\
\hline$\Delta \rho_{\max }, \Delta \rho_{\min }\left(\mathrm{e} \AA^{-3}\right)$ & $2.06,-2.34$ \\
\hline
\end{tabular}

Computer programs: Apex3 v2018.1-0 (Bruker, 2018), SAINT V8.38A (Bruker, 2016), SHELXS97 (Sheldrick, 2008),

SHELXL2018/3 (Sheldrick, 2015, 2018), SHELXLE Rev1030 (Hübschle et al., 2011). 


\section{Refinement details:}

One of the ligand side chains and a solvate methylene chloride molecule were refined as disordered. The major and minor moieties were each restrained to have similar geometries. $U^{\mathrm{ij}}$ components of ADPs for disordered atoms closer to each other than $2.0 \AA$ were restrained to be similar. Subject to these conditions the occupancy ratio refined to $0.543(4)$ to $0.457(4)$ for the side chain, and to $0.657(8)$ to $0.343(8)$ for the solvate molecule.

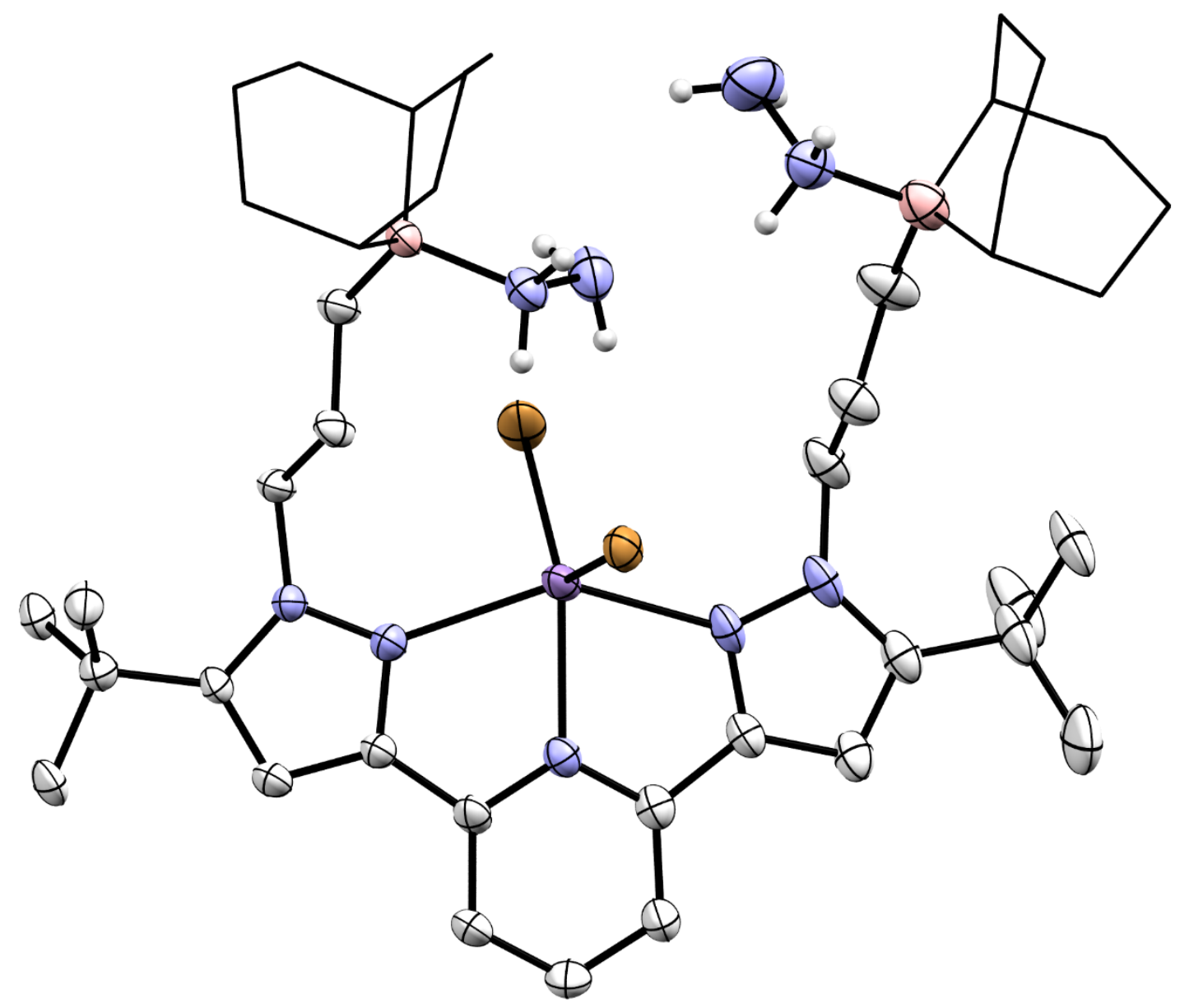

Figure S87 Molecular structure of ( $\left.{ }^{\mathrm{BBN} P D P}{ }^{\mathrm{BB} u}\right) \mathrm{MnBr}_{2}\left(\mathrm{~N}_{2} \mathrm{H}_{4}\right)$ displayed with $50 \%$ probability ellipsoids. Hydrogen atoms not attached to heteroatoms, disorder and solvate molecules are omitted for clarity. The 9-BBN substituents are displayed in wireframe for clarity. A second asymmetric molecule in the unit cell is not shown. 
Complex: ( $\left.{ }^{\mathrm{BBN} P D P^{t B u}}\right) \mathrm{MnBr}_{2}\left(\mathrm{NH}_{3}\right)_{2}$

Local name: jk313

CCDC 1993639

Table S10 Crystallographic experimental parameters for ( $\left.{ }^{\mathrm{BBN}} \mathrm{PDP}^{\mathrm{tBu}}\right) \mathrm{MnBr}_{2}\left(\mathrm{NH}_{3}\right)_{2}$.

\begin{tabular}{|c|c|}
\hline \multicolumn{2}{|l|}{ Crystal data } \\
\hline Chemical formula & $\mathrm{C}_{41} \mathrm{H}_{69} \mathrm{~B}_{2} \mathrm{Br}_{2} \mathrm{MnN} \mathrm{N}_{7} \cdot 1.414\left(\mathrm{CH}_{2} \mathrm{Cl}_{2}\right)$ \\
\hline$M r$ & 1016.64 \\
\hline Crystal system, space group & Monoclinic, $P 2_{1} / n$ \\
\hline Temperature (K) & 150 \\
\hline$a, b, c(\AA)$ & 16.7053 (7), 25.2417 (11), 24.7261 (11) \\
\hline$\beta\left(^{\circ}\right)$ & $109.3671(15)$ \\
\hline$V\left(\AA^{3}\right)$ & 9836.3 (7) \\
\hline$Z$ & 8 \\
\hline Radiation type & Mo $K \alpha$ \\
\hline$\mu\left(\mathrm{mm}^{-1}\right)$ & 2.08 \\
\hline Crystal size $(\mathrm{mm})$ & $0.45 \times 0.42 \times 0.32$ \\
\hline \multicolumn{2}{|l|}{ Data collection } \\
\hline Diffractometer & Bruker AXS D8 Quest CMOS diffractometer \\
\hline Absorption correction & $\begin{array}{l}\text { Multi-scan SADABS 2016/2: Krause, L., Herbst-Irmer, R., Sheldrick } \\
\text { G.M. \& Stalke D., J. Appl. Cryst. } 48 \text { (2015) 3-10 }\end{array}$ \\
\hline$T_{\min }, T_{\max }$ & $0.655,0.746$ \\
\hline $\begin{array}{l}\text { No. of measured, independent and } \\
\text { observed }[I>2 \mathrm{~s}(I)] \text { reflections }\end{array}$ & $124551,27673,21612$ \\
\hline$R_{\text {int }}$ & 0.048 \\
\hline$(\sin \theta / \lambda)_{\max }\left(\AA^{-1}\right)$ & 0.715 \\
\hline \multicolumn{2}{|l|}{ Refinement } \\
\hline$R\left[F^{2}>2 \sigma\left(F^{2}\right)\right], w R\left(F^{2}\right), S$ & $0.047,0.120,1.06$ \\
\hline No. of reflections & 27673 \\
\hline No. of parameters & 1195 \\
\hline No. of restraints & 436 \\
\hline \multirow[t]{2}{*}{$\mathrm{H}$-atom treatment } & $\mathrm{H}$-atom parameters constrained \\
\hline & $\begin{array}{l}w=1 /\left[\mathrm{s}^{2}\left(F_{\mathrm{o}}^{2}\right)+(0.0413 P)^{2}+18.3033 P\right] \\
\text { where } P=\left(F_{\mathrm{o}}^{2}+2 F_{\mathrm{c}}^{2}\right) / 3\end{array}$ \\
\hline$\Delta \rho_{\max }, \Delta \rho_{\min }\left(\mathrm{e} \AA^{-3}\right)$ & $0.82,-0.91$ \\
\hline
\end{tabular}

Computer programs: Apex3 v2016.9-0 (Bruker, 2016), SAINT V8.37A (Bruker, 2016), SHELXS97

(Sheldrick, 2008), SHELXL2017/1 (Sheldrick, 2015, 2017), SHELXLE Rev859 (Hübschle et al., 2011). 


\section{Refinement details:}

The structure was solved by isomorphous replacement from its iron counterpart. The solvent model was slightly changed.

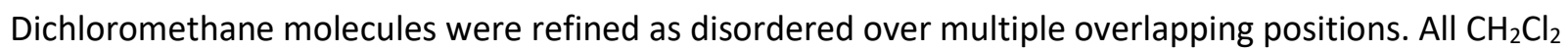
moieties were restrained to have similar geometries, and $\mathrm{U}^{\mathrm{ij}}$ components were restrained to be similar for atoms closer to each other than $2.0 \AA$. No attempts were made to sum up the occupancy of overlapping molecules to unity and sites are slightly deficient in total molecule count (indicating possible disorder with diffuse other solvent molecules (pentane was used in the crystallizations)). A slight antibumping restraint was applied to avoid close $\mathrm{H}$... H contacts to disordered solvate molecules. Actual occupancies for all moieties are $0.59(2), 0.33(2), 0.632(4), 0.635(8), 0.027(3), 0.272(7), 0.299(4)$ and 0.044(3).

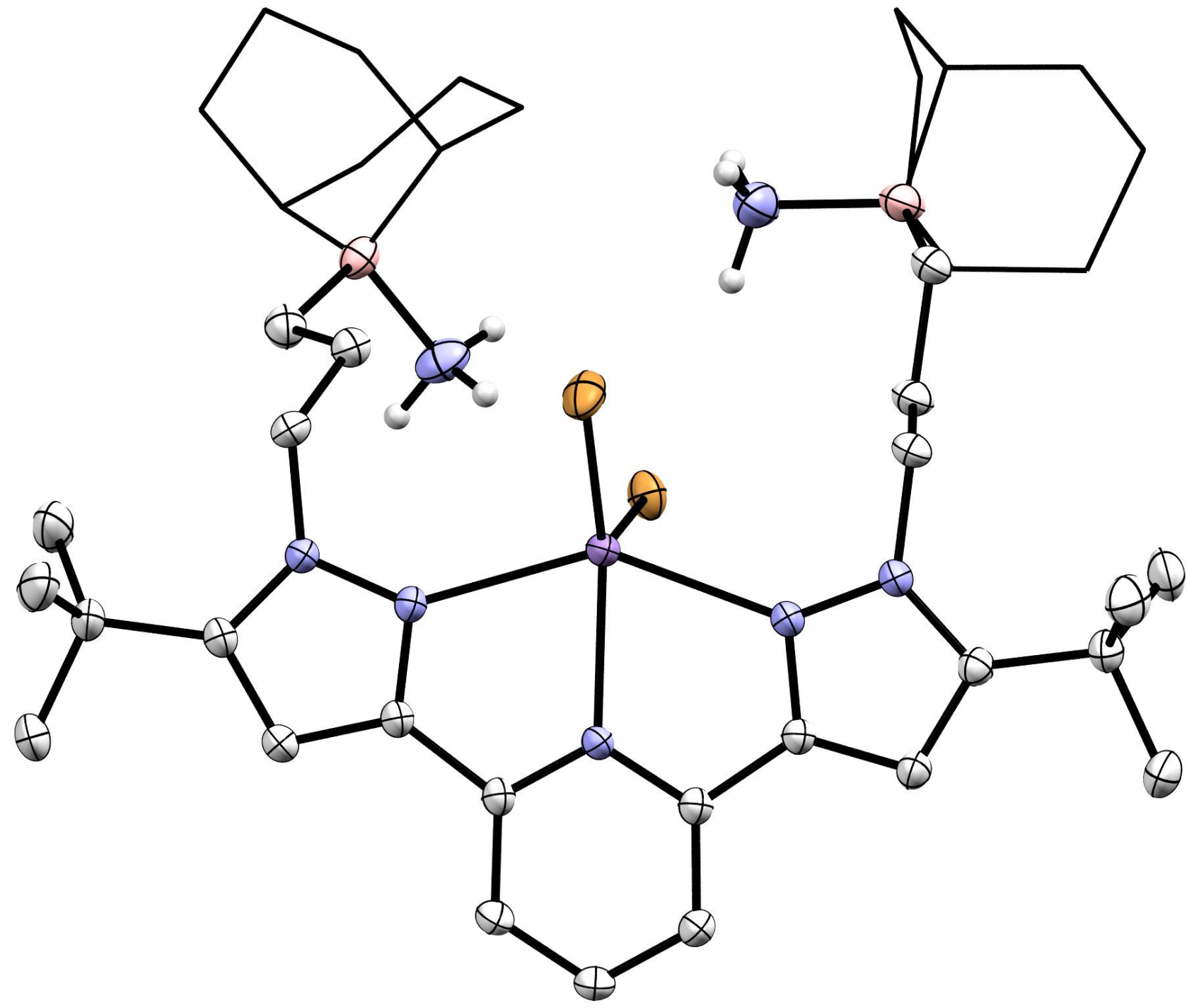

Figure $\mathbf{S 8 8}$ Molecular structure of ( $\left.{ }^{\mathrm{BBN} P D P}{ }^{\mathrm{tBu}}\right) \mathrm{MnBr}_{2}\left(\mathrm{NH}_{3}\right)_{2}$ displayed with $50 \%$ probability ellipsoids. Hydrogen atoms not attached to heteroatoms and solvate molecules are omitted for clarity. The 9-BBN substituents are displayed in wireframe for clarity. 
Complex: ( $\left.{ }^{\mathrm{BBN} P D P}{ }^{t \mathrm{Bu}}\right) \mathrm{CoBr}_{2}\left(\mathrm{NH}_{3}\right)_{2}$

Local name: jk314

CCDC 1993638

Table S11 Crystallographic experimental parameters for ( $\left.{ }^{\mathrm{BBN} P D P}{ }^{\mathrm{BB} u}\right) \mathrm{CoBr}_{2}\left(\mathrm{NH}_{3}\right)_{2}$.

\begin{tabular}{|c|c|}
\hline \multicolumn{2}{|l|}{ Crystal data } \\
\hline Chemical formula & $\mathrm{C}_{41} \mathrm{H}_{69} \mathrm{~B}_{2} \mathrm{Br}_{2} \mathrm{CoN}_{7} \cdot 1.225\left(\mathrm{CH}_{2} \mathrm{Cl}_{2}\right)$ \\
\hline$M r$ & 1004.64 \\
\hline Crystal system, space group & Monoclinic, $P 2_{1} / n$ \\
\hline Temperature (K) & 150 \\
\hline$a, b, c(\AA)$ & 16.7106 (8), $25.2218(15), 24.5911(14)$ \\
\hline$\beta\left(^{\circ}\right)$ & $109.6080(19)$ \\
\hline$V\left(\AA^{3}\right)$ & $9763.4(9)$ \\
\hline$Z$ & 8 \\
\hline Radiation type & Mo $K \alpha$ \\
\hline$\mu\left(\mathrm{mm}^{-1}\right)$ & 2.16 \\
\hline Crystal size (mm) & $0.55 \times 0.53 \times 0.45$ \\
\hline \multicolumn{2}{|l|}{ Data collection } \\
\hline Diffractometer & Bruker AXS D8 Quest CMOS diffractometer \\
\hline Absorption correction & $\begin{array}{l}\text { Multi-scan SADABS 2016/2: Krause, L., Herbst-Irmer, R., Sheldrick } \\
\text { G.M. \& Stalke D., J. Appl. Cryst. } 48 \text { (2015) 3-10 }\end{array}$ \\
\hline$T_{\min }, T_{\max }$ & $0.511,0.747$ \\
\hline $\begin{array}{l}\text { No. of measured, independent and } \\
\text { observed }[I>2 s(I)] \text { reflections }\end{array}$ & 120591, 29262, 21276 \\
\hline$R_{\text {int }}$ & 0.040 \\
\hline$(\sin \theta / \lambda)_{\max }\left(\AA^{-1}\right)$ & 0.735 \\
\hline \multicolumn{2}{|l|}{ Refinement } \\
\hline$R\left[F^{2}>2 \sigma\left(F^{2}\right)\right], w R\left(F^{2}\right), S$ & $0.047,0.137,1.04$ \\
\hline No. of reflections & 29262 \\
\hline No. of parameters & 1167 \\
\hline No. of restraints & 309 \\
\hline \multirow[t]{2}{*}{$\mathrm{H}$-atom treatment } & $\mathrm{H}$-atom parameters constrained \\
\hline & $\begin{array}{l}w=1 /\left[\mathrm{s}^{2}\left(F_{\mathrm{o}}^{2}\right)+(0.0675 P)^{2}+11.1268 P\right] \\
\text { where } P=\left(F_{\mathrm{o}}^{2}+2 F_{\mathrm{c}}^{2}\right) / 3\end{array}$ \\
\hline$\Delta \rho_{\max }, \Delta \rho_{\min }\left(\mathrm{e} \AA^{-3}\right)$ & $1.46,-1.17$ \\
\hline
\end{tabular}

Computer programs: Apex3 v2016.9-0 (Bruker, 2016), SAINT V8.37A (Bruker, 2016), SHELXS97

(Sheldrick, 2008), SHELXL2017/1 (Sheldrick, 2015, 2017), SHELXLE Rev859 (Hübschle et al., 2011). 


\section{Refinement details:}

The structure was solved by isomorphous replacement from its iron counterpart. The solvent model was slightly changed.

Dichloromethane molecules were refined as disordered over multiple overlapping positions. All $\mathrm{CH}_{2} \mathrm{Cl}_{2}$ moieties were restrained to have similar geometries, and $\mathrm{U}^{\mathrm{ij}}$ components were restrained to be similar for atoms closer to each other than $2.0 \AA$. No attempts were made to sum up the occupancy of overlapping molecules to unity and sites are slightly deficient in total molecule count (indicating possible disorder with diffuse other solvent molecules (pentane was used in the crystallizations)). Actual occupancies for all moieties are 0.57(2), 0.21(2), 0.581(12), 0.497(14), 0.130(4), 0.327(14) and 0.136(12).

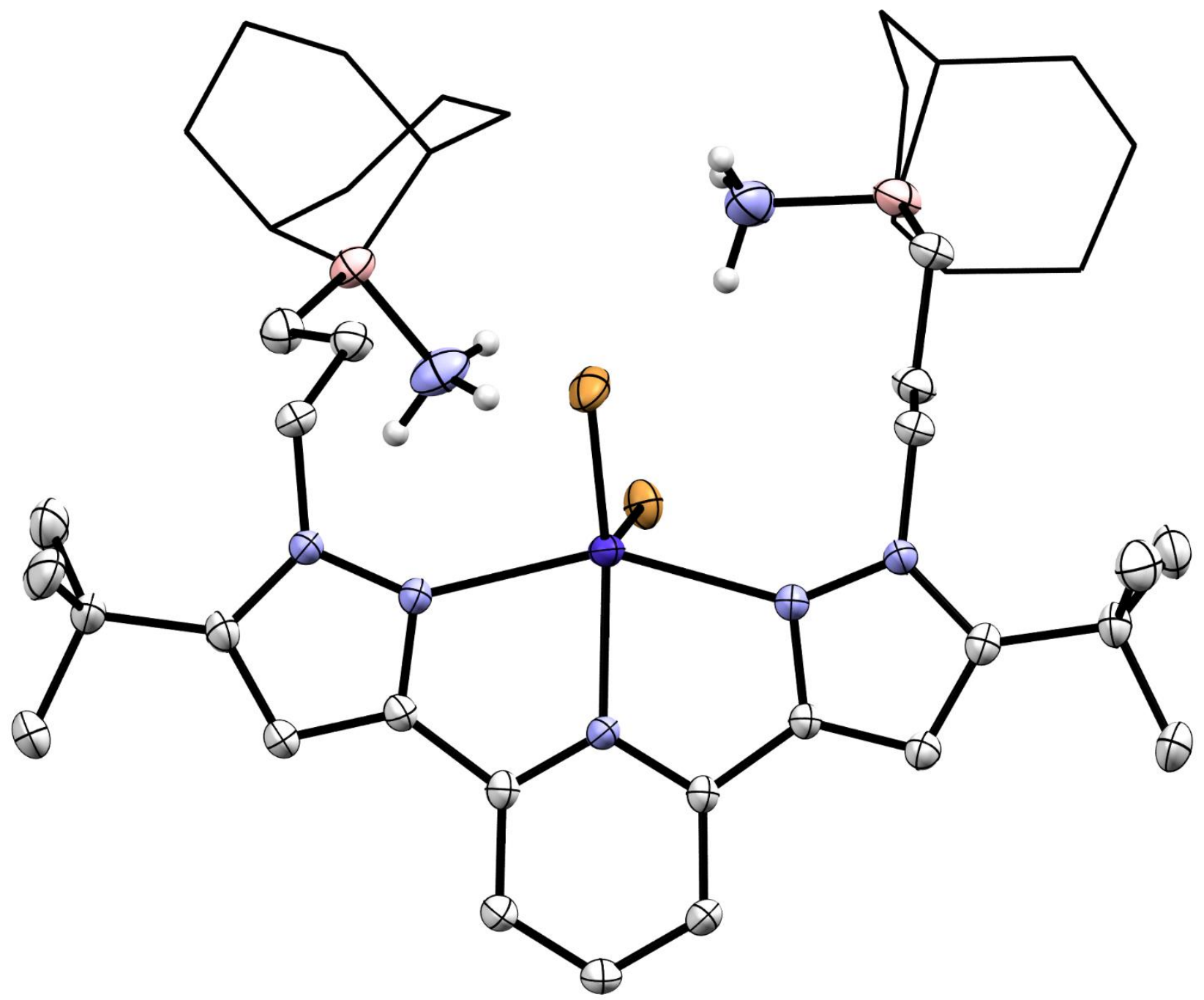

Figure S89 Molecular structure of ( $\left.{ }^{\mathrm{BBN}} \mathrm{PDP}{ }^{\mathrm{tBu}}\right) \mathrm{CoBr}_{2}\left(\mathrm{NH}_{3}\right)_{2}$ displayed with $50 \%$ probability ellipsoids. Hydrogen atoms not attached to heteroatoms and solvate molecules are omitted for clarity. The 9-BBN substituents are displayed in wireframe for clarity. 
Complex: ( $\left.{ }^{\mathrm{BBN} P D P}{ }^{\mathrm{tBu}}\right) \mathrm{FeBr}_{2}\left(\mathrm{NH}_{3}\right)_{2}$

Local name: jk268

CCDC 1993640

Table S12 Crystallographic experimental parameters for ( $\left.{ }^{\mathrm{BBN} P D P}{ }^{\mathrm{BB} u}\right) \mathrm{FeBr}_{2}\left(\mathrm{NH}_{3}\right)_{2}$.

\begin{tabular}{|c|c|}
\hline \multicolumn{2}{|l|}{ Crystal data } \\
\hline Chemical formula & $\mathrm{C}_{41} \mathrm{H}_{69} \mathrm{~B}_{2} \mathrm{Br}_{2} \mathrm{FeN}_{7} \cdot 1.299\left(\mathrm{CH}_{2} \mathrm{Cl}_{2}\right)$ \\
\hline$M r$ & 1007.67 \\
\hline Crystal system, space group & Monoclinic, $P 2_{1} / n$ \\
\hline Temperature (K) & 150 \\
\hline$a, b, c(\AA)$ & 16.7015 (6), $25.2466(10), 24.6426(10)$ \\
\hline$\beta\left({ }^{\circ}\right)$ & $109.6890(14)$ \\
\hline$V\left(\AA^{3}\right)$ & $9783.2(7)$ \\
\hline$Z$ & 8 \\
\hline Radiation type & Mo $K \alpha$ \\
\hline$\mu\left(\mathrm{mm}^{-1}\right)$ & 2.12 \\
\hline Crystal size (mm) & $0.46 \times 0.31 \times 0.30$ \\
\hline \multicolumn{2}{|l|}{ Data collection } \\
\hline Diffractometer & Bruker AXS D8 Quest CMOS diffractometer \\
\hline Absorption correction & $\begin{array}{l}\text { Multi-scan SADABS 2016/2: Krause, L., Herbst-Irmer, R., Sheldrick G.M. } \\
\text { \& Stalke D., J. Appl. Cryst. } 48 \text { (2015) 3-10 }\end{array}$ \\
\hline$T_{\min }, T_{\max }$ & $0.577,0.747$ \\
\hline $\begin{array}{l}\text { No. of measured, independent and } \\
\text { observed }[I>2 s(I)] \text { reflections }\end{array}$ & $132227,34707,22034$ \\
\hline$R_{\text {int }}$ & 0.053 \\
\hline$(\sin \theta / \lambda)_{\max }\left(\AA^{-1}\right)$ & 0.771 \\
\hline \multicolumn{2}{|l|}{ Refinement } \\
\hline$R\left[F^{2}>2 \sigma\left(F^{2}\right)\right], w R\left(F^{2}\right), S$ & $0.050,0.156,1.03$ \\
\hline No. of reflections & 34707 \\
\hline No. of parameters & 1157 \\
\hline No. of restraints & 292 \\
\hline $\mathrm{H}$-atom treatment & $\mathrm{H}$-atom parameters constrained \\
\hline$\Delta \rho_{\max }, \Delta \rho_{\min }\left(\mathrm{e} \AA^{-3}\right)$ & $1.31,-1.20$ \\
\hline
\end{tabular}

Computer programs: Apex3 v2016.9-0 (Bruker, 2016), SAINT V8.37A (Bruker, 2016), SHELXS97

(Sheldrick, 2008), SHELXL2016/6 (Sheldrick, 2015, 2016), SHELXLE Rev714 (Hübschle et al., 2011). 


\section{Refinement details:}

Dichloromethane molecules were refined as disordered over multiple overlapping positions. All $\mathrm{CH}_{2} \mathrm{Cl}_{2}$ moieties were restrained to have similar geometries, and $\mathrm{U}^{\mathrm{ij}}$ components were restrained to be similar for atoms closer to each other than $2.0 \AA$. No attempts were made to sum up the occupancy of overlapping molecules to unity and sites are slightly deficient in total molecule count (indicating possible disorder with diffuse other solvent molecules (pentane was used in the crystallizations)). Actual occupancies for all moieties are $0.55(2), 0.30(2), 0.677(4), 0.528(13), 0.068(4), 0.306(13)$ and $0.171(4)$.

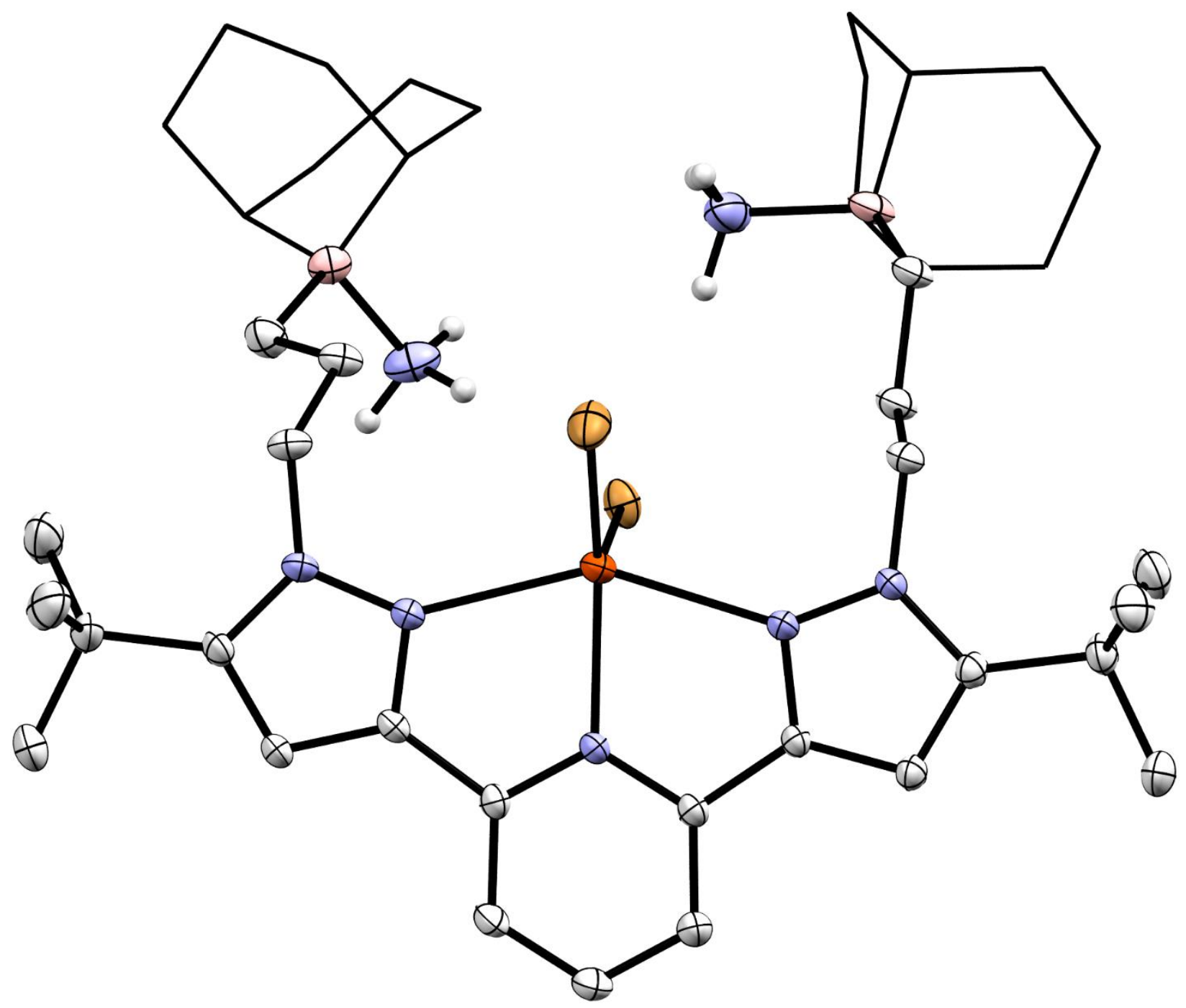

Figure $\mathbf{S 9 0}$ Molecular structure of $\left({ }^{\mathrm{BBN} P D P}{ }^{\mathrm{BBu}}\right) \mathrm{FeBr}_{2}\left(\mathrm{NH}_{3}\right)_{2}$ displayed with $50 \%$ probability ellipsoids. Hydrogen atoms not attached to heteroatoms and solvate molecules are omitted for clarity. The 9-BBN substituents are displayed in wireframe for clarity. 
Complex: $\left({ }^{\left.\mathrm{BBN} P D P^{\mathrm{BBu}}\right)} \mathrm{ZnCl}_{2}\left(\mathrm{NH}_{3}\right)_{2}\right.$

Local name: jk2134

CCDC 1993631

Table S13 Crystallographic experimental parameters for ( $\left.{ }^{\mathrm{BBN} P D P}{ }^{\mathrm{BB} u}\right) \mathrm{ZnCl}_{2}\left(\mathrm{NH}_{3}\right)_{2}$.

\begin{tabular}{|c|c|}
\hline \multicolumn{2}{|l|}{ Crystal data } \\
\hline Chemical formula & $\mathrm{C}_{41} \mathrm{H}_{69} \mathrm{~B}_{2} \mathrm{Cl}_{2} \mathrm{~N} 7 \mathrm{Zn} \cdot[+$ solvent $]$ \\
\hline Mr & 817.92 \\
\hline Crystal system, space group & Monoclinic, C2/c \\
\hline Temperature (K) & 150 \\
\hline$a, b, c(\AA)$ & 16.8056 (7), $25.1343(12), 24.2367$ (12) \\
\hline$\beta\left(^{\circ}\right)$ & $109.772(2)$ \\
\hline$V\left(\AA^{3}\right)$ & $9634.0(8)$ \\
\hline$z$ & 8 \\
\hline Radiation type & Cu $K \alpha$ \\
\hline$\mu\left(\mathrm{mm}^{-1}\right)$ & 1.98 \\
\hline Crystal size (mm) & $0.15 \times 0.14 \times 0.09$ \\
\hline \multicolumn{2}{|l|}{ Data collection } \\
\hline Diffractometer & Bruker AXS D8 Quest CMOS diffractometer \\
\hline Absorption correction & $\begin{array}{l}\text { Multi-scan SADABS 2016/2: Krause, L., Herbst-Irmer, R., Sheldrick } \\
\text { G.M. \& Stalke D., J. Appl. Cryst. } 48 \text { (2015) 3-10 }\end{array}$ \\
\hline$T_{\min }, T_{\max }$ & $0.570,0.754$ \\
\hline $\begin{array}{l}\text { No. of measured, independent and } \\
\text { observed }[I>2 s(I)] \text { reflections }\end{array}$ & $25172,9359,8385$ \\
\hline$R_{\text {int }}$ & 0.035 \\
\hline$(\sin \theta / \lambda)_{\max }\left(\AA^{-1}\right)$ & 0.638 \\
\hline \multicolumn{2}{|l|}{ Refinement } \\
\hline$R\left[F^{2}>2 \sigma\left(F^{2}\right)\right], w R\left(F^{2}\right), S$ & $0.036,0.095,1.05$ \\
\hline No. of reflections & 9359 \\
\hline No. of parameters & 678 \\
\hline No. of restraints & 738 \\
\hline $\mathrm{H}$-atom treatment & H-atom parameters constrained \\
\hline$\Delta \rho_{\max }, \Delta \rho_{\min }\left(\mathrm{e} \AA^{-3}\right)$ & $0.42,-0.37$ \\
\hline
\end{tabular}

Computer programs: Apex3 v2016.9-0 (Bruker, 2016), SAINT V8.37A (Bruker, 2016), SHELXS97

(Sheldrick, 2008), SHELXL2017/1 (Sheldrick, 2017), SHELXLE Rev859 (Hübschle et al., 2011). 


\section{Refinement details:}

The structure contains 4 independent solvent accessible voids of $1442 \AA^{3}$ combined. The residual electron density peaks, originating mostly from heavily disordered pentane molecules, were not arranged in an interpretable pattern. The cif and fcf files were thus corrected for using reverse Fourier transform methods using the SQUEEZE routine (P. van der Sluis, A.L. Spek Acta Cryst. 1990, A46, 194201) as implemented in the program Platon. The resultant files were used in the further refinement. (The FAB file with details of the SQUEEZE results is appended to the cif file). The SQUEEZE procedure corrected for 367 electrons within the solvent accessible voids.

Both ligand side arms are disordered, with equal occupancy ratio (the disorder of side arm 2 is causing the less pronounced disorder of side arm 1). All disordered moieties were restrained to have similar geometries. $U^{i j}$ components of ADPs for disordered atoms closer to each other than $2.0 \AA$ were restrained to be similar. Subject to these conditions the occupancy ratio refined to $0.517(3)$ to $0.517(3)$.

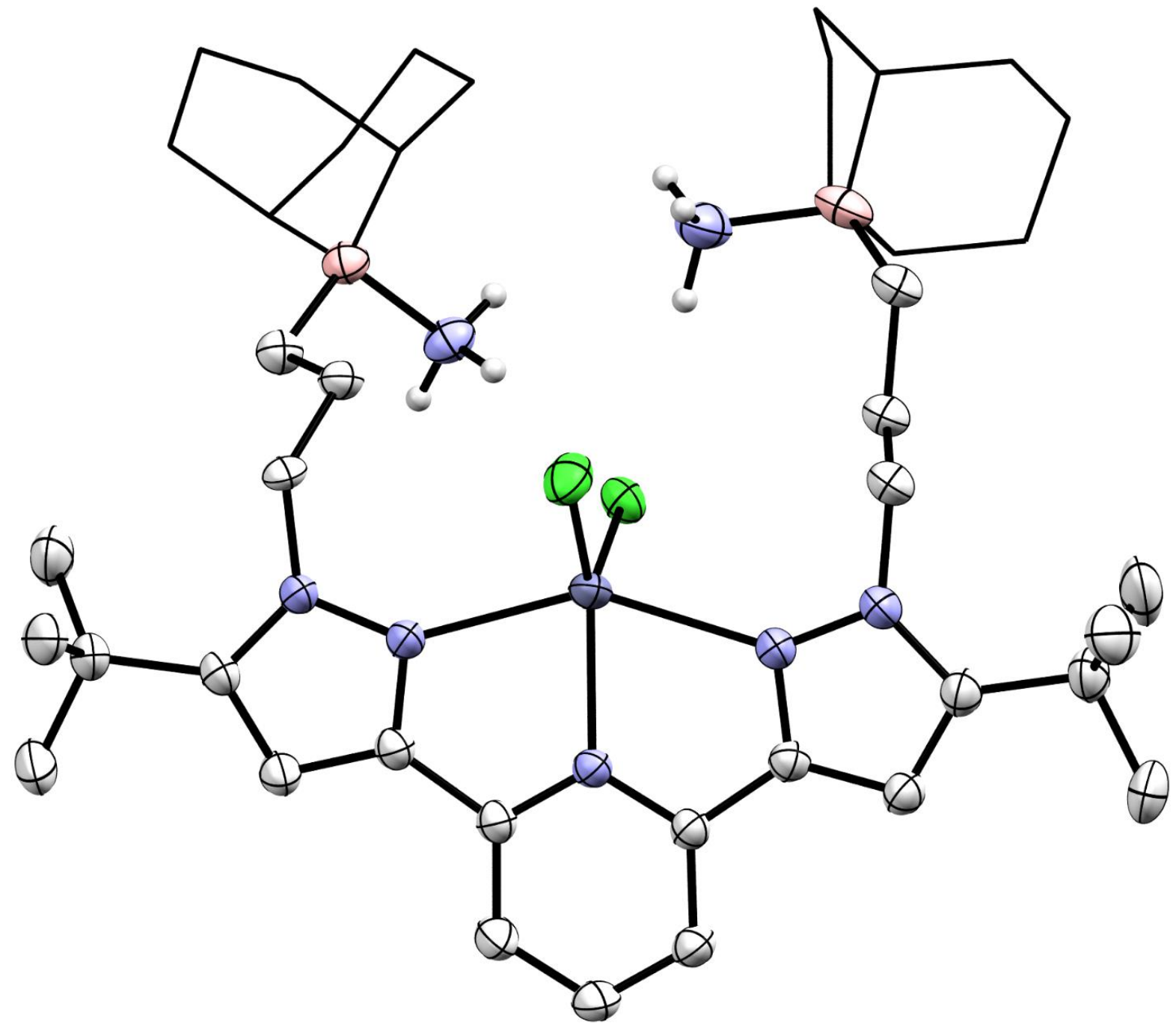

Figure S91 Molecular structure of ( $\left.{ }^{\mathrm{BBN} P D P}{ }^{\mathrm{tBu}}\right) \mathrm{ZnCl}_{2}\left(\mathrm{NH}_{3}\right)_{2}$ displayed with $50 \%$ probability ellipsoids. Hydrogen atoms not attached to heteroatoms and disorder are omitted for clarity. The 9-BBN substituents are displayed in wireframe for clarity. 
Complex: $\left({ }^{\mathrm{BBN} P D P}{ }^{\mathrm{BBu}}\right) \mathrm{VCl}_{2}\left(\mathrm{NH}_{3}\right)_{2}(\mathrm{THF})$

Local name: jk4283

CCDC 1993632

Table S14 Crystallographic experimental parameters for ( $\left.{ }^{\mathrm{BBN} P D P}{ }^{\mathrm{tBu}}\right) \mathrm{VCl}_{2}\left(\mathrm{NH}_{3}\right)_{2}(\mathrm{THF})$.

\begin{tabular}{|c|c|}
\hline \multicolumn{2}{|l|}{ Crystal data } \\
\hline Chemical formula & $\mathrm{C}_{45} \mathrm{H}_{77} \mathrm{~B}_{2} \mathrm{Cl}_{2} \mathrm{~N} 7 \mathrm{OV} \cdot[+$ solvent $]$ \\
\hline$M r$ & 875.59 \\
\hline Crystal system, space group & Monoclinic, $C 2 / c$ \\
\hline Temperature (K) & 150 \\
\hline$a, b, c(\AA)$ & $29.7239(13), 17.7320(7), 21.4883(10)$ \\
\hline$\beta\left({ }^{\circ}\right)$ & $94.4563(18)$ \\
\hline$V\left(\AA^{3}\right)$ & $11291.5(8)$ \\
\hline$Z$ & 8 \\
\hline Radiation type & $\mathrm{Cu} K \alpha$ \\
\hline$\mu\left(\mathrm{mm}^{-1}\right)$ & 2.60 \\
\hline Crystal size (mm) & $0.32 \times 0.30 \times 0.11$ \\
\hline \multicolumn{2}{|l|}{ Data collection } \\
\hline Diffractometer & $\begin{array}{l}\text { Bruker AXS D8 Quest CMOS diffractometer with Photonll charge- } \\
\text { integrating pixel array detector (CPAD) }\end{array}$ \\
\hline Absorption correction & $\begin{array}{l}\text { Multi-scan SADABS 2016/2: Krause, L., Herbst-Irmer, R., Sheldrick } \\
\text { G.M. \& Stalke D., J. Appl. Cryst. } 48 \text { (2015) 3-10 }\end{array}$ \\
\hline$T_{\min }, T_{\max }$ & $0.558,0.753$ \\
\hline $\begin{array}{l}\text { No. of measured, independent and } \\
\text { observed }[I>2 \mathrm{~s}(I)] \text { reflections }\end{array}$ & $31606,10591,9590$ \\
\hline$R_{\text {int }}$ & 0.034 \\
\hline$(\sin \theta / \lambda)_{\max }\left(\AA^{-1}\right)$ & 0.610 \\
\hline \multicolumn{2}{|l|}{ Refinement } \\
\hline$R\left[F^{2}>2 \sigma\left(F^{2}\right)\right], w R\left(F^{2}\right), S$ & $0.049,0.145,1.07$ \\
\hline No. of reflections & 10591 \\
\hline No. of parameters & 568 \\
\hline No. of restraints & 160 \\
\hline \multirow[t]{2}{*}{$\mathrm{H}$-atom treatment } & $\mathrm{H}$-atom parameters constrained \\
\hline & $w=1 /\left[\mathrm{s}^{2}\left(F_{\mathrm{o}}{ }^{2}\right)+(0.0812 P)^{2}+11.9475 P\right]$ where $P=\left(F_{\mathrm{o}}{ }^{2}+2 F_{\mathrm{c}}{ }^{2}\right) / 3$ \\
\hline$\Delta \rho_{\max }, \Delta \rho_{\min }\left(\mathrm{e} \AA^{-3}\right)$ & $0.79,-0.35$ \\
\hline
\end{tabular}

Computer programs: Apex3 v2018.7-2 (Bruker, 2017), SAINT V8.38A (Bruker, 2016), SHELXS97 (Sheldrick, 2008),

SHELXL2018/3 (Sheldrick, 2015, 2018), SHELXLE Rev946 (Hübschle et al., 2011). 


\section{Refinement details:}

The carbon atoms of a vanadium coordinated THF molecule were refined as disordered over two sites. The two disordered moieties were restrained to have similar geometries. $\mathrm{U}^{\mathrm{ij}}$ components of ADPs for disordered atoms closer to each other than $2.0 \AA$ Aere restrained to be similar. Indications for very minor additional disorder were ignored. Subject to these conditions the occupancy ratio refined to $0.519(8)$ to $0.481(8)$.

The structure contains 4 independent solvent accessible voids of $2508 \AA^{3}$ combined. The residual electron density peaks were arranged in a pattern indicating extensive multiple disorder of diethyl ether molecules.

The disorder was beyond what can be reasonably unambiguously refined and the structure factors were instead augmented via reverse Fourier transform methods using the SQUEEZE routine (P. van der Sluis, A.L. Spek Acta Cryst. 1990, A46, 194-201) as implemented in the program Platon. The resultant FAB file containing the structure factor contribution from the electron content of the void space was used together with the original hkl file in the further refinement. (The FAB file with details of the SQUEEZE results is appended to this cif file). The SQUEEZE procedure corrected for 596 electrons within the solvent accessible voids, equal to ca. 14 diethyl ether molecules per unit cell.

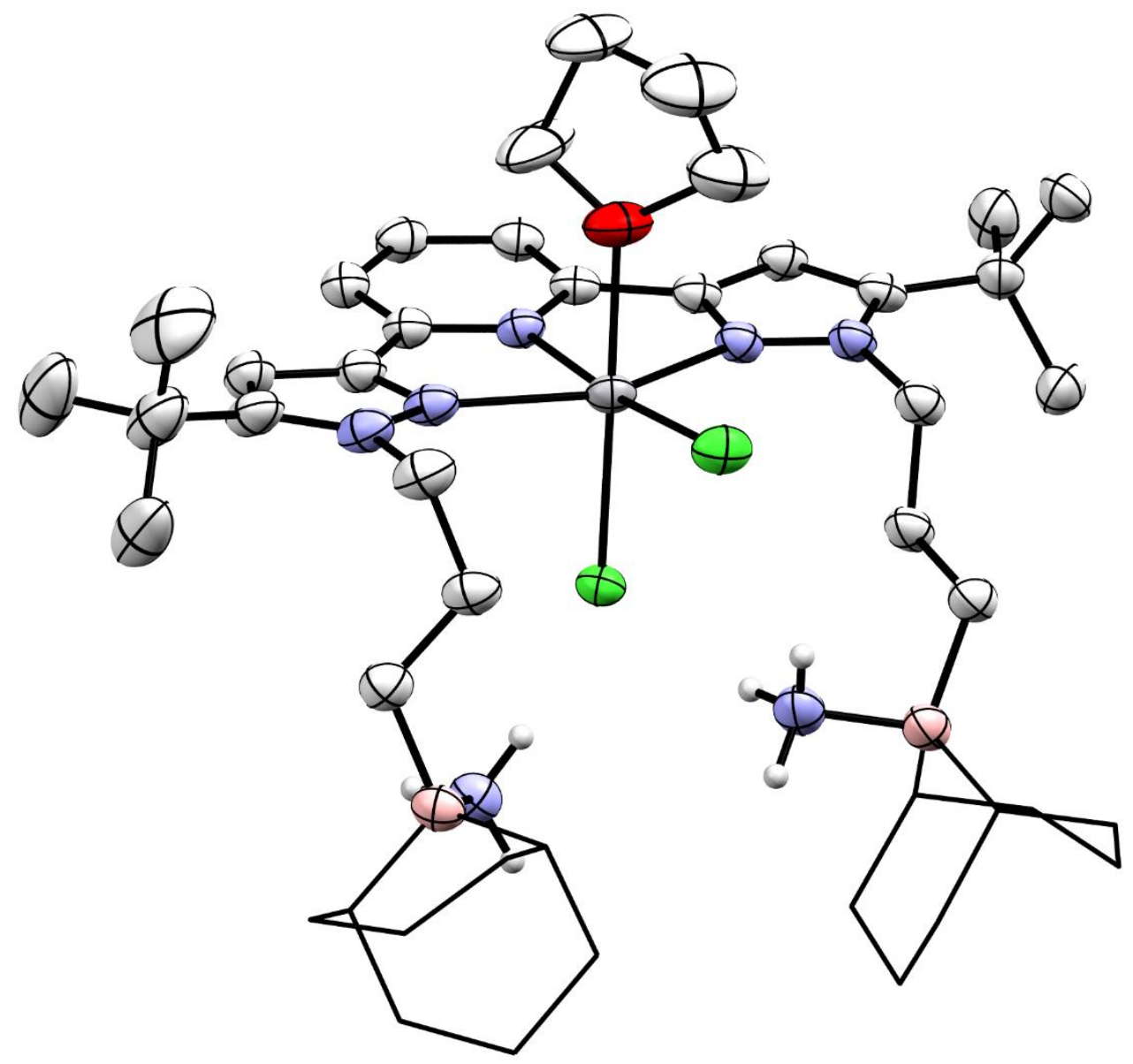

Figure $\mathbf{S 9 2}$ Molecular structure of ( $\left.{ }^{\mathrm{BBN} P D P}{ }^{\mathrm{tBu}}\right) \mathrm{VCl}_{2}\left(\mathrm{NH}_{3}\right)_{2}(\mathrm{THF})$ displayed with $50 \%$ probability ellipsoids. Hydrogen atoms not attached to heteroatoms and disorder are omitted for clarity. The 9-BBN substituents are displayed in wireframe for clarity. 
Complex: ( $\left.{ }^{\mathrm{BBN}} \mathrm{PDP}{ }^{\mathrm{tBu}}\right) \mathrm{FeBr}_{2}\left(\mathrm{NH}_{2} \mathrm{Ph}\right)_{2}$

Local name: jk1240

CCDC 1993627

Table S15 Crystallographic experimental parameters for ( $\left.{ }^{\mathrm{BBN}} \mathrm{PDP}^{\mathrm{tBu}}\right) \mathrm{FeBr}_{2}\left(\mathrm{NH}_{2} \mathrm{Ph}\right)_{2}$.

\begin{tabular}{|c|c|}
\hline \multicolumn{2}{|l|}{ Crystal data } \\
\hline Chemical formula & $\mathrm{C}_{53} \mathrm{H}_{77} \mathrm{~B}_{2} \mathrm{Br}_{2} \mathrm{FeN}_{7} \cdot 2.5\left(\mathrm{C}_{6} \mathrm{H}_{6}\right)$ \\
\hline Mr & 1244.77 \\
\hline Crystal system, space group & Triclinic, $P \overline{1}$ \\
\hline Temperature (K) & 85 \\
\hline$a, b, c(\AA)$ & 11.0983 (2), $16.7421(4), 17.3032$ (4) \\
\hline$\alpha, \beta, \gamma\left({ }^{\circ}\right)$ & $86.765(2), 89.057(2), 88.029(2)$ \\
\hline$V\left(\AA^{3}\right)$ & $3207.73(12)$ \\
\hline$z$ & 2 \\
\hline Radiation type & $\mathrm{Cu} K \alpha$ \\
\hline$\mu\left(\mathrm{mm}^{-1}\right)$ & 3.68 \\
\hline Crystal size (mm) & $0.16 \times 0.05 \times 0.01$ \\
\hline \multicolumn{2}{|l|}{ Data collection } \\
\hline Diffractometer & Abstract diffractometer \\
\hline Absorption correction & $\begin{array}{l}\text { Multi-scan CrysAlis PRO 1.171.38.41 (Rigaku Oxford Diffraction, } \\
\text { 2015) Empirical absorption correction using spherical harmonics, } \\
\text { implemented in SCALE3 ABSPACK scaling algorithm. }\end{array}$ \\
\hline$T_{\min }, T_{\max }$ & $0.543,1.000$ \\
\hline $\begin{array}{l}\text { No. of measured, independent and } \\
\text { observed }[I>2 \mathrm{~s}(I)] \text { reflections }\end{array}$ & $49085,49085,42477$ \\
\hline$R_{\text {int }}$ & 0.048 \\
\hline$(\sin \theta / \lambda)_{\max }\left(\AA^{-1}\right)$ & 0.607 \\
\hline \multicolumn{2}{|l|}{ Refinement } \\
\hline$R\left[F^{2}>2 \sigma\left(F^{2}\right)\right], w R\left(F^{2}\right), S$ & $0.058,0.180,1.07$ \\
\hline No. of reflections & 49085 \\
\hline No. of parameters & 728 \\
\hline \multirow[t]{2}{*}{$\mathrm{H}$-atom treatment } & H-atom parameters constrained \\
\hline & $\begin{array}{l}w=1 /\left[\mathrm{s}^{2}\left(F_{\mathrm{o}}^{2}\right)+(0.0439 P)^{2}+15.6875 P\right] \\
\text { where } P=\left(F_{\mathrm{o}}^{2}+2 F_{\mathrm{c}}^{2}\right) / 3\end{array}$ \\
\hline$\Delta \rho_{\max }, \Delta \rho_{\min }\left(\mathrm{e} \AA^{-3}\right)$ & $1.55,-1.05$ \\
\hline
\end{tabular}

Computer programs: CrysAlis PRO 1.171.38.41 (Rigaku OD, 2015), SHELXS97 (Sheldrick, 2008), SHELXL2014/7 (Sheldrick, 2014). 


\section{Refinement details:}

Refined as a 2-component twin. The crystal under investigation was found to be non-merohedrically twinned. Rigaku programs compatible with the diffractometer (twinsolve) gave unsatisfactory results. With no usable data set obtainable through simultaneous integration of both twin domains, the data were instead handled as if not twinned, with only the major domain integrated, and converted into an hklf 5 type format hkl file after integration using the "Make HKLF5 File" routine as implemented in WinGX.

The twin law matrix was obtained using the program ROTAX as implemented in WinGX and corresponds to a 180 degree rotation around the 100 direct lattice direction, the twin matrix was:

$1 \quad 0 \quad 0$

$0.104-10$

$0.052 \quad 0-1$

The Overlap R1 and R2 values in the "Make HKLF5 File" routine used were both 0.08, i.e. reflections with a discriminator function less or equal to overlap radius of 0.08 were counted overlapped, all others as single. The discriminator function used was the "delta function on index non-integrality". No reflections were omitted.

The structure was solved using direct methods with all reflections of component 1 . The structure was refined using the hklf 5 routine with all reflections of component 1 (including the overlapping ones) as obtained from WinGX, resulting in a BASF value of 0.41625 . No Rint value is obtainable for the hklf 5 type file using the WinGX routine.

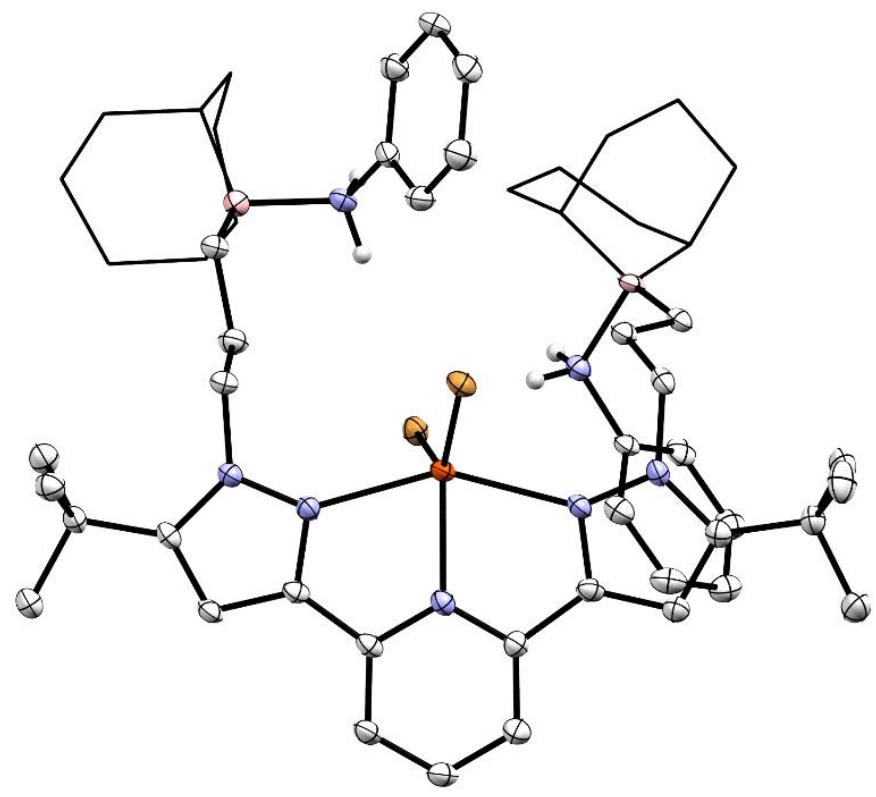

Figure $\mathbf{S 9 3}$ Molecular structure of ( $\left.{ }^{\mathrm{BBN} P D P}{ }^{\mathrm{tBu}}\right) \mathrm{FeBr}_{2}\left(\mathrm{NH}_{2} \mathrm{Ph}\right)_{2}$ displayed with $50 \%$ probability ellipsoids. Hydrogen atoms not attached to heteroatoms and solvate molecules are omitted for clarity. The 9-BBN substituents are displayed in wireframe for clarity. Select distances $(\AA)$ : $\mathrm{Fe}-\mathrm{Br}=2.4934(10), 2.4304(9)$; Fe- $\mathrm{N}_{\mathrm{pyr}}=2.095(5) ; \mathrm{Fe}-\mathrm{N}_{\mathrm{pz}}=2.261(5), 2.245(5) ; \mathrm{B}-\mathrm{N}=1.710(7), 1.694(8)$. 
Complex: $\left({ }^{\mathrm{BBN} P D P}{ }^{\mathrm{tBu}}\right) \mathrm{Mn}\left(\mathrm{NH}_{2}\right)_{2}$

Local name: jk320b

CCDC 1993635

Table S16 Crystallographic experimental parameters for ( $\left.{ }^{\mathrm{BBN}} \mathrm{PDP} \mathrm{P}^{\mathrm{tBu}}\right) \mathrm{Mn}\left(\mathrm{NH}_{2}\right)_{2}$.

\begin{tabular}{|c|c|}
\hline \multicolumn{2}{|l|}{ Crystal data } \\
\hline Chemical formula & $\mathrm{C}_{41} \mathrm{H}_{67} \mathrm{~B}_{2} \mathrm{MnN}_{7} \cdot \mathrm{CH}_{2} \mathrm{Cl}_{2}$ \\
\hline$M r$ & 819.50 \\
\hline Crystal system, space group & Tetragonal, $P_{4} 2{ }_{1} 2$ \\
\hline Temperature (K) & 150 \\
\hline$a, c(\AA ̊)$ & $16.2567(7), 16.5068(8)$ \\
\hline$V\left(\AA^{3}\right)$ & $4362.4(4)$ \\
\hline$z$ & 4 \\
\hline Radiation type & Mo $K \alpha$ \\
\hline$\mu\left(\mathrm{mm}^{-1}\right)$ & 0.46 \\
\hline Crystal size $(\mathrm{mm})$ & $0.16 \times 0.15 \times 0.11$ \\
\hline \multicolumn{2}{|l|}{ Data collection } \\
\hline Diffractometer & Bruker AXS D8 Quest CMOS diffractometer \\
\hline Absorption correction & $\begin{array}{l}\text { Multi-scan SADABS 2016/2: Krause, L., Herbst-Irmer, R., } \\
\text { Sheldrick G.M. \& Stalke D., J. Appl. Cryst. } 48 \text { (2015) 3-10 }\end{array}$ \\
\hline$T_{\min }, T_{\max }$ & $0.645,0.746$ \\
\hline $\begin{array}{l}\text { No. of measured, independent and } \\
\text { observed }[I>2 \mathrm{~s}(I)] \text { reflections }\end{array}$ & $22066,5973,5426$ \\
\hline Rint & 0.050 \\
\hline$(\sin \theta / \lambda)_{\max }\left(\AA^{-1}\right)$ & 0.714 \\
\hline \multicolumn{2}{|l|}{ Refinement } \\
\hline$R\left[F^{2}>2 \sigma\left(F^{2}\right)\right], w R\left(F^{2}\right), S$ & $0.038,0.088,1.06$ \\
\hline No. of reflections & 5973 \\
\hline No. of parameters & 261 \\
\hline No. of restraints & 25 \\
\hline $\mathrm{H}$-atom treatment & $\mathrm{H}$-atom parameters constrained \\
\hline$\Delta \rho_{\max }, \Delta \rho_{\min }\left(\mathrm{e} \AA^{-3}\right)$ & $0.29,-0.40$ \\
\hline Absolute structure & $\begin{array}{l}\text { Flack } x \text { determined using } 2041 \text { quotients }[(I+)-(I-)] /[(I+)+(I-)] \\
\text { (Parsons, Flack and Wagner, Acta Cryst. B69 (2013) 249-259). }\end{array}$ \\
\hline Absolute structure parameter & $-0.009(7)$ \\
\hline
\end{tabular}

Computer programs: Apex3 v2016.9-0 (Bruker, 2016), SAINT V8.37A (Bruker, 2016), SHELXS97

(Sheldrick, 2008), SHELXL2017/1 (Sheldrick, 2015, 2017), SHELXLE Rev859 (Hübschle et al., 2011). 


\section{Refinement details:}

A methylene chloride molecule is located on a twofold axis, and disordered by flipping around the $\mathrm{Cl}$...Cl axis. The two $\mathrm{C}-\mathrm{Cl}$ distances were restrained to be similar, the ADPs of the two $\mathrm{C}$ atoms constrained to be identical, and $\mathrm{U}^{\mathrm{ij}}$ components of ADPs for disordered atoms closer to each other than $2.0 \AA$ were restrained to be similar. Subject to these conditions the occupancy ratio refined to $0.647(8)$ to $0.353(8)$.

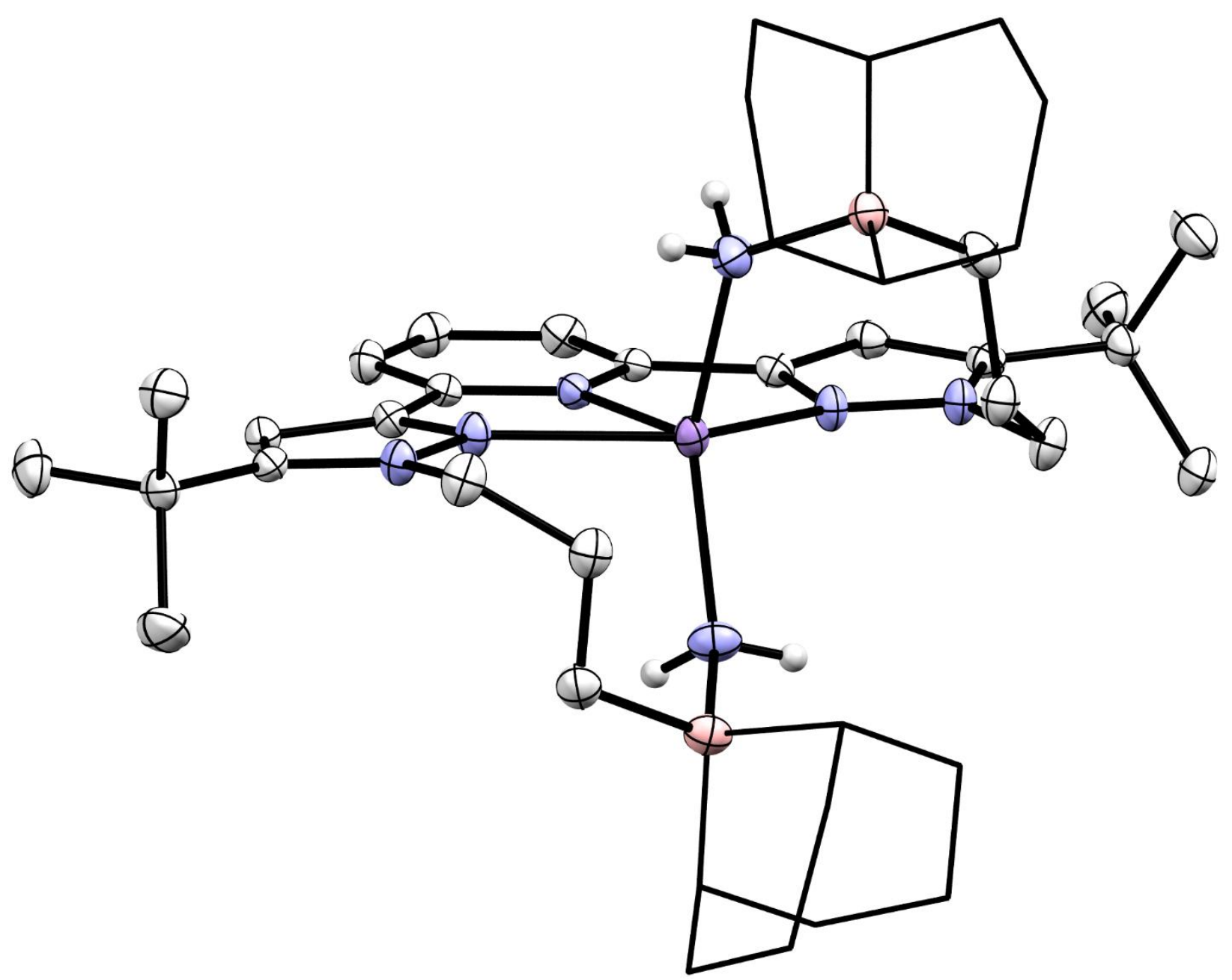

Figure $\mathbf{S 9 4}$ Molecular structure of ( $\left.{ }^{\mathrm{BBN} P D P}{ }^{\mathrm{Bu}}\right) \mathrm{Mn}\left(\mathrm{NH}_{2}\right)_{2}$ displayed with $50 \%$ probability ellipsoids. Hydrogen atoms not attached to heteroatoms and solvate molecules are omitted for clarity. The 9-BBN substituents are displayed in wireframe for clarity. 
Complex: ( $\left.{ }^{\mathrm{BBN} P D P^{\mathrm{BBu}}}\right) \mathrm{Co}\left(\mathrm{NH}_{2}\right)_{2}$

Local name: jk315

CCDC 1993641

Table S17 Crystallographic experimental parameters for $\left({ }^{\mathrm{BBN}} \mathrm{PDP}{ }^{\mathrm{tBu}}\right) \mathrm{Co}\left(\mathrm{NH}_{2}\right)_{2}$.

\begin{tabular}{|c|c|}
\hline \multicolumn{2}{|l|}{ Crystal data } \\
\hline Chemical formula & $\mathrm{C}_{41} \mathrm{H}_{67} \mathrm{~B}_{2} \mathrm{CoN}_{7} \cdot \mathrm{CH}_{2} \mathrm{Cl}_{2}$ \\
\hline Mr & 823.49 \\
\hline Crystal system, space group & Tetragonal, $P 4_{1} 2{ }_{1} 2$ \\
\hline Temperature (K) & 150 \\
\hline$a, c(\AA ̊)$ & $16.1338(7), 16.6024(7)$ \\
\hline$V\left(\AA^{3}\right)$ & $4321.6(4)$ \\
\hline$Z$ & 4 \\
\hline Radiation type & Mo $K \alpha$ \\
\hline$\mu\left(\mathrm{mm}^{-1}\right)$ & 0.56 \\
\hline Crystal size $(\mathrm{mm})$ & $0.10 \times 0.10 \times 0.10$ \\
\hline \multicolumn{2}{|l|}{ Data collection } \\
\hline Diffractometer & Bruker AXS D8 Quest CMOS diffractometer \\
\hline Absorption correction & $\begin{array}{l}\text { Multi-scan SADABS 2016/2: Krause, L., Herbst-Irmer, R., Sheldrick } \\
\text { G.M. \& Stalke D., J. Appl. Cryst. } 48 \text { (2015) 3-10 }\end{array}$ \\
\hline$T_{\min }, T_{\max }$ & $0.719,0.746$ \\
\hline $\begin{array}{l}\text { No. of measured, independent and } \\
\text { observed }[I>2 s(I)] \text { reflections }\end{array}$ & $211675,6925,6436$ \\
\hline Rint & 0.036 \\
\hline$(\sin \theta / \lambda)_{\max }\left(\AA^{-1}\right)$ & 0.726 \\
\hline \multicolumn{2}{|l|}{ Refinement } \\
\hline$R\left[F^{2}>2 \sigma\left(F^{2}\right)\right], w R\left(F^{2}\right), S$ & $0.034,0.095,1.09$ \\
\hline No. of reflections & 6925 \\
\hline No. of parameters & 278 \\
\hline No. of restraints & 39 \\
\hline $\mathrm{H}$-atom treatment & $\mathrm{H}$-atom parameters constrained \\
\hline$\Delta \rho_{\max }, \Delta \rho_{\min }\left(\mathrm{e} \AA^{-3}\right)$ & $0.45,-0.42$ \\
\hline Absolute structure & $\begin{array}{l}\text { Flack } x \text { determined using } 2709 \text { quotients }[(I+)-(I-)] /[(I+)+(I-)] \\
\text { (Parsons, Flack and Wagner, Acta Cryst. B69 (2013) 249-259). }\end{array}$ \\
\hline Absolute structure parameter & $-0.0158(18)$ \\
\hline
\end{tabular}

Computer programs: Apex3 v2016.9-0 (Bruker, 2016), SAINT V8.37A (Bruker, 2016), SHELXS97

(Sheldrick, 2008), SHELXL2017/1 (Sheldrick, 2015, 2017), SHELXLE Rev853 (Hübschle et al., 2011). 


\section{Refinement details:}

A methylene chloride molecule is disordered over three positions. One position is located on a two fold axis, with the $\mathrm{C}$ atom of one moiety located exactly on the axis, the $\mathrm{C}$ atom of the other slightly offset, creating two symmetry equivalent moieties. The $\mathrm{C}-\mathrm{Cl}$ distances of all three moieties were restrained to be similar, and the $U^{i j}$ components of ADPs of disordered atoms closer to each other than $2.0 \AA$ to be similar to each other. Occupancy rates refined to $0.463(10)$ and two times $0.269(5)$.

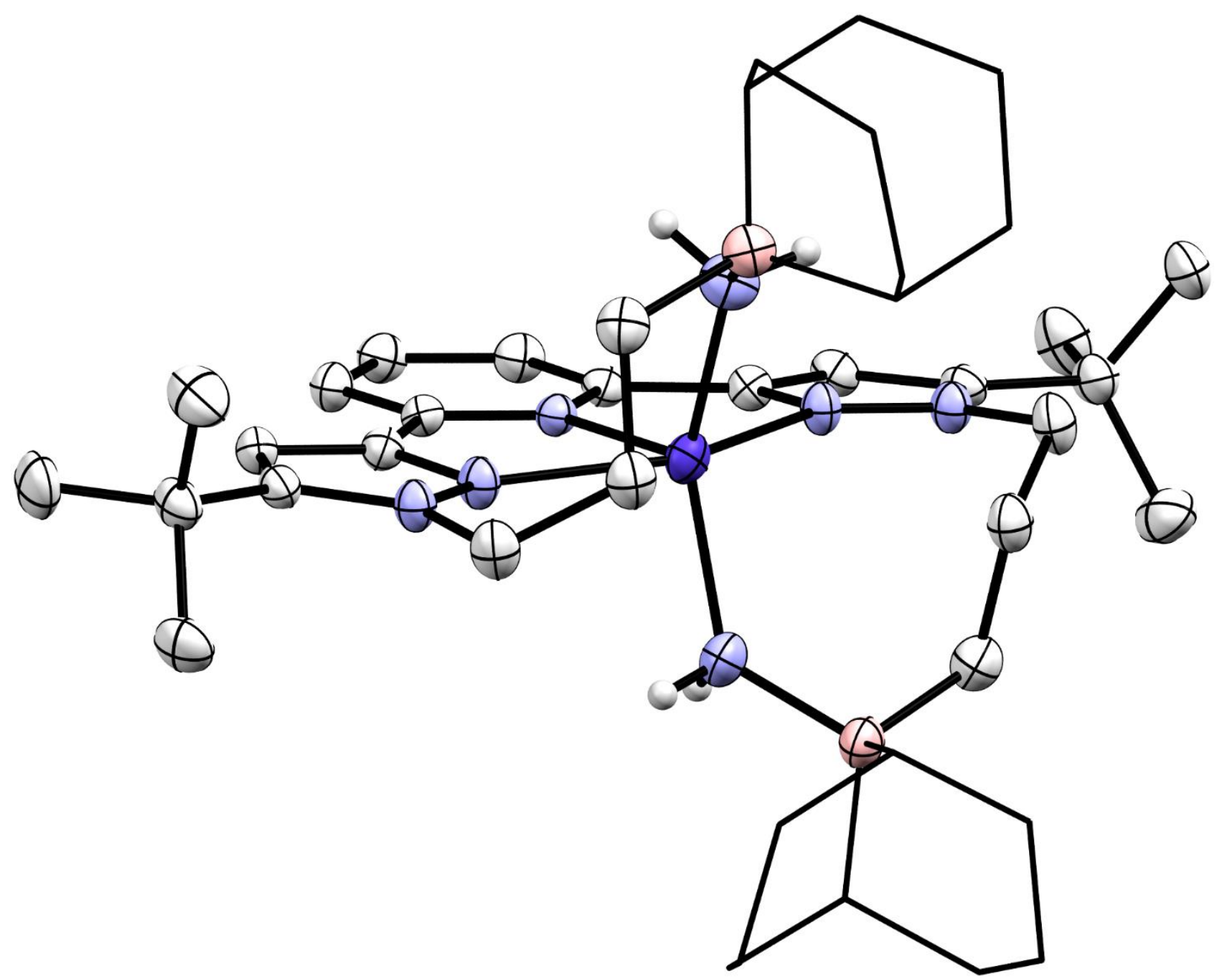

Figure $\mathbf{S 9 5}$ Molecular structure of $\left({ }^{\mathrm{BBN} P D P}{ }^{\mathrm{BB} u}\right) \mathrm{Co}\left(\mathrm{NH}_{2}\right)_{2}$ displayed with $50 \%$ probability ellipsoids.

Hydrogen atoms not attached to heteroatoms and solvate molecules are omitted for clarity. The 9-BBN substituents are displayed in wireframe for clarity. 
Complex: ( $\left.\left.{ }^{\mathrm{BBN} P D P^{t B u}}\right) \mathrm{ZnNH}_{2}\right)_{2}$

Local name: jk2151

CCDC 1993634

Table S18 Crystallographic experimental parameters for ( $\left.{ }^{\mathrm{BBN} P D P}{ }^{\mathrm{BB} u}\right) \mathrm{Zn}\left(\mathrm{NH}_{2}\right)_{2}$.

\begin{tabular}{|c|c|}
\hline \multicolumn{2}{|l|}{ Crystal data } \\
\hline Chemical formula & $\mathrm{C}_{41} \mathrm{H}_{67} \mathrm{~B}_{2} \mathrm{~N} 7 \mathrm{Zn} \cdot \mathrm{CH}_{2} \mathrm{Cl}_{2}$ \\
\hline Mr & 829.93 \\
\hline Crystal system, space group & Tetragonal, $P 4_{3} 2{ }_{1} 2$ \\
\hline Temperature (K) & 150 \\
\hline$a, c(\AA ̊)$ & $16.1659(3), 16.5883(4)$ \\
\hline$V\left(\AA^{3}\right)$ & 4335.12 (19) \\
\hline$z$ & 4 \\
\hline Radiation type & $\mathrm{Cu} K \alpha$ \\
\hline$\mu\left(\mathrm{mm}^{-1}\right)$ & 2.20 \\
\hline Crystal size (mm) & $0.18 \times 0.16 \times 0.09$ \\
\hline \multicolumn{2}{|l|}{ Data collection } \\
\hline Diffractometer & Bruker AXS D8 Quest CMOS diffractometer \\
\hline Absorption correction & $\begin{array}{l}\text { Multi-scan SADABS 2016/2: Krause, L., Herbst-Irmer, R., } \\
\text { Sheldrick G.M. \& Stalke D., J. Appl. Cryst. } 48 \text { (2015) 3-10 }\end{array}$ \\
\hline$T_{\min }, T_{\max }$ & $0.486,0.754$ \\
\hline $\begin{array}{l}\text { No. of measured, independent and } \\
\text { observed }[l>2 \mathrm{~s}(I)] \text { reflections }\end{array}$ & $12187,4413,4279$ \\
\hline$R_{\text {int }}$ & 0.041 \\
\hline$(\sin \theta / \lambda)_{\max }\left(\AA^{-1}\right)$ & 0.638 \\
\hline \multicolumn{2}{|l|}{ Refinement } \\
\hline$R\left[F^{2}>2 \sigma\left(F^{2}\right)\right], w R\left(F^{2}\right), S$ & $0.038,0.099,1.02$ \\
\hline No. of reflections & 4413 \\
\hline No. of parameters & 264 \\
\hline No. of restraints & 19 \\
\hline $\mathrm{H}$-atom treatment & $\mathrm{H}$-atom parameters constrained \\
\hline$\Delta \rho_{\max }, \Delta \rho_{\min }\left(\mathrm{e} \AA^{-3}\right)$ & $0.98,-0.37$ \\
\hline Absolute structure & $\begin{array}{l}\text { Flack } x \text { determined using } 1725 \text { quotients }[(I+)-(I-)] /[(I+)+(I-)] \\
\text { (Parsons, Flack and Wagner, Acta Cryst. B69 (2013) 249-259). }\end{array}$ \\
\hline Absolute structure parameter & $0.005(9)$ \\
\hline
\end{tabular}

Computer programs: Apex3 v2016.9-0 (Bruker, 2016), SAINT V8.37A (Bruker, 2016), SHELXS97

(Sheldrick, 2008), SHELXL2017/1 (Sheldrick, 2017), SHELXLE Rev859 (Hübschle et al., 2011). 


\section{Refinement details:}

A methylene chloride molecule is disordered over two orientations (both moieties are located on a twofold rotation axis). The $\mathrm{C}-\mathrm{Cl}$ distances were restrained to be similar, and $\mathrm{U}^{\mathrm{ij}}$ components of ADPs for disordered atoms closer to each other than $2.0 \AA \AA$ were restrained to be similar. Subject to these conditions the occupancy ratio refined to 0.460 (14) to $0.540(14)$.

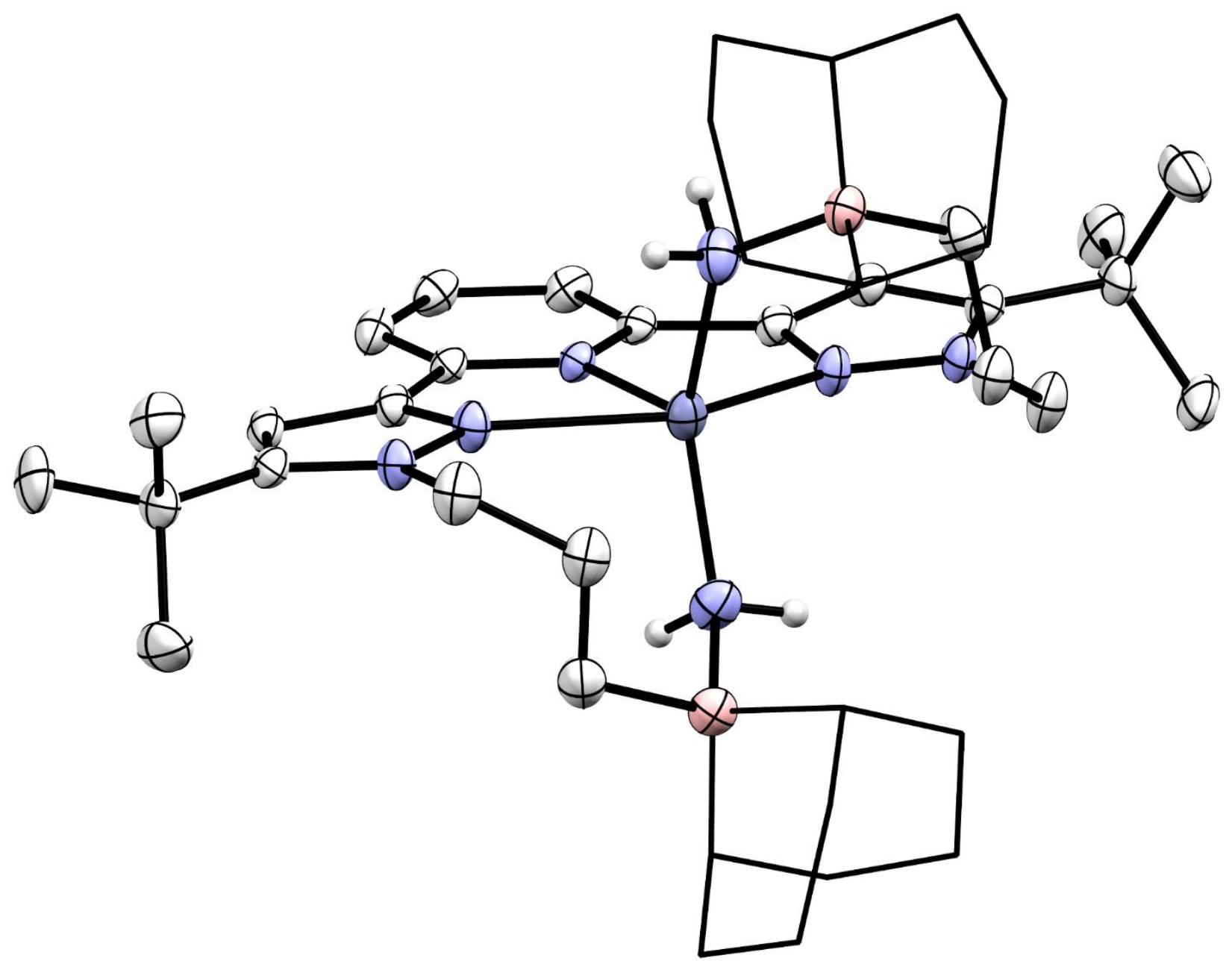

Figure S96 Molecular structure of ${ }^{\left.\mathrm{BBN} P D P^{\mathrm{BB}}\right)} \mathrm{Zn}\left(\mathrm{NH}_{2}\right)_{2}$ displayed with $50 \%$ probability ellipsoids. Hydrogen atoms not attached to heteroatoms and solvate molecules are omitted for clarity. The 9-BBN substituents are displayed in wireframe for clarity. 
Complex: [Cs(18-crown-6) $\left.)_{2}\right]\left[\left({ }^{\mathrm{BBN} P D P}{ }^{\mathrm{tBu}}\right) \mathrm{Fe}\left(\mathrm{NH}_{2}\right)_{2}\right]$

Local name: jk425A

CCDC 1993636

Table S19 Crystallographic experimental parameters for [Cs(18-crown-6 $\left.)_{2}\right]\left[\left({ }^{B B N} P D P{ }^{t B u}\right) \mathrm{Fe}\left(\mathrm{NH}_{2}\right)_{2}\right]$.

\begin{tabular}{|c|c|}
\hline \multicolumn{2}{|l|}{ Crystal data } \\
\hline Chemical formula & $\mathrm{C}_{41} \mathrm{H}_{67} \mathrm{~B}_{2} \mathrm{FeNN}_{7} \cdot \mathrm{C}_{24} \mathrm{H}_{48} \mathrm{CsO}_{12}$ \\
\hline Mr & 1397.01 \\
\hline Crystal system, space group & Hexagonal, $P 6522$ \\
\hline Temperature (K) & 150 \\
\hline$a, c(\AA ̊)$ & $14.2169(2), 60.3681(11)$ \\
\hline$V\left(\AA^{3}\right)$ & $10566.9(4)$ \\
\hline$Z$ & 6 \\
\hline Radiation type & $\mathrm{Cu} K \alpha$ \\
\hline$\mu\left(\mathrm{mm}^{-1}\right)$ & 6.18 \\
\hline Crystal size $(\mathrm{mm})$ & $0.18 \times 0.15 \times 0.05$ \\
\hline \multicolumn{2}{|l|}{ Data collection } \\
\hline Diffractometer & Bruker AXS D8 Quest CMOS diffractometer \\
\hline Absorption correction & $\begin{array}{l}\text { Multi-scan SADABS 2016/2: Krause, L., Herbst-Irmer, R., Sheldrick } \\
\text { G.M. \& Stalke D., J. Appl. Cryst. } 48 \text { (2015) 3-10 }\end{array}$ \\
\hline$T_{\min }, T_{\max }$ & $0.258,0.473$ \\
\hline $\begin{array}{l}\text { No. of measured, independent and } \\
\text { observed }[I>2 \mathrm{~s}(I)] \text { reflections }\end{array}$ & $18459,7075,6604$ \\
\hline Rint & 0.030 \\
\hline$(\sin \theta / \lambda)_{\max }\left(\AA^{-1}\right)$ & 0.640 \\
\hline \multicolumn{2}{|l|}{ Refinement } \\
\hline$R\left[F^{2}>2 \sigma\left(F^{2}\right)\right], w R\left(F^{2}\right), S$ & $0.037,0.095,1.05$ \\
\hline No. of reflections & 7075 \\
\hline No. of parameters & 514 \\
\hline No. of restraints & 356 \\
\hline $\mathrm{H}$-atom treatment & $\mathrm{H}$-atom parameters constrained \\
\hline$\Delta \rho_{\max }, \Delta \rho_{\min }\left(\mathrm{e} \AA^{-3}\right)$ & $1.05,-0.46$ \\
\hline Absolute structure & $\begin{array}{l}\text { Flack } x \text { determined using } 2380 \text { quotients }[(I+)-(I-)] /[(I+)+(I-)] \\
\text { (Parsons, Flack and Wagner, Acta Cryst. B69 (2013) 249-259). }\end{array}$ \\
\hline Absolute structure parameter & $-0.008(2)$ \\
\hline
\end{tabular}

Computer programs: Apex3 v2017.3-0 (Bruker, 2016), SAINT V8.37A (Bruker, 2016), SHELXS97

(Sheldrick, 2008), SHELXL2018/3 (Sheldrick, 2015, 2018), SHELXLE Rev909 (Hübschle et al., 2011). 


\section{Refinement details:}

The borane fragment and surrounding atoms are disordered, with differing directions of rotation that the fragment wraps around the iron center. The two disordered moieties were restrained to have similar geometries. Major and minor atoms $\mathrm{C} 11$ were restrained to be coplanar with the adjacent to the $\mathrm{N}_{2} \mathrm{C}_{3}$ ring. $\mathrm{U}^{\mathrm{ij}}$ components of ADPs for disordered atoms closer to each other than $2.0 \AA$ were restrained to be similar. Subject to these conditions the occupancy ratio refined to $0.821(4)$ to $0.179(4)$.

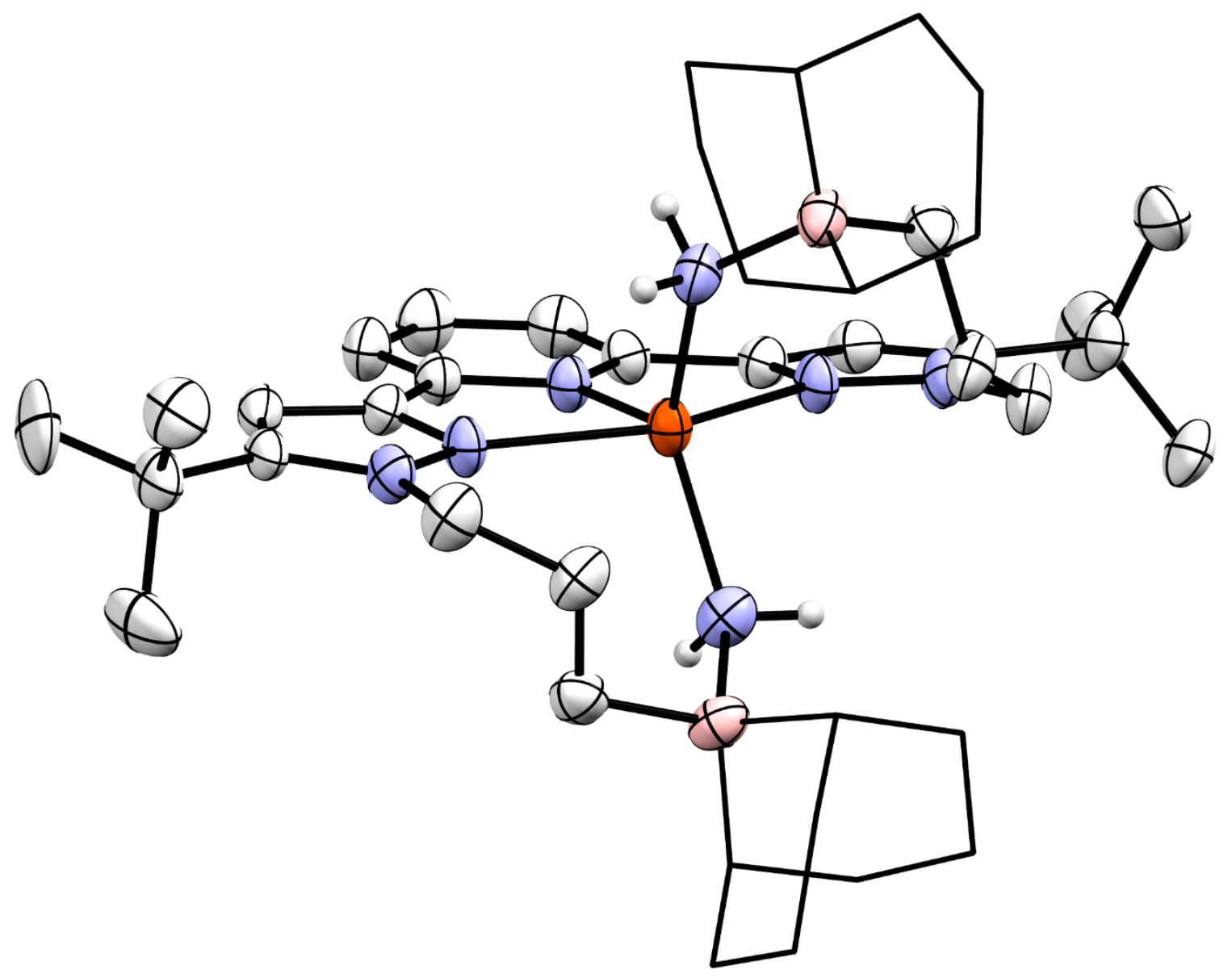

Figure S97 Molecular structure of [Cs(18-crown-6 $\left.)_{2}\right]\left[\left({ }^{\mathrm{BBN} P D P}{ }^{\mathrm{Bu} u}\right) \mathrm{Fe}\left(\mathrm{NH}_{2}\right)_{2}\right]$ displayed with $50 \%$ probability ellipsoids. Hydrogen atoms not attached to heteroatoms and disorder are omitted for clarity. The cationic portion of the molecule is omitted for clarity. The 9-BBN substituents are displayed in wireframe for clarity. 
Complex: [K(2,2,2-cryptand)][(benzyl- $\left.\left.{ }^{B B N} P D P{ }^{t B u}\right) \mathrm{Fe}\left(\mathrm{NH}_{2}\right)_{2}\right]$

Local name: jk4181

CCDC 1993643

Table S20 Crystallographic experimental parameters for [K(2,2,2-cryptand)][(benzyl- $\left.\left.{ }^{\mathrm{BBN}}{ }^{\mathrm{PDP}}{ }^{\mathrm{tBu}}\right) \mathrm{Fe}\left(\mathrm{NH}_{2}\right)_{2}\right]$.

\begin{tabular}{|c|c|}
\hline \multicolumn{2}{|l|}{ Crystal data } \\
\hline Chemical formula & $\mathrm{C}_{48} \mathrm{H}_{74} \mathrm{~B}_{2} \mathrm{FeN}_{7} \cdot \mathrm{C}_{18} \mathrm{H}_{36} \mathrm{KN}_{2} \mathrm{O}_{6} \cdot 2\left(\mathrm{C}_{4} \mathrm{H}_{8} \mathrm{O}\right)$ \\
\hline$M r$ & 1386.40 \\
\hline Crystal system, space group & Monoclinic, $P 2_{1} / n$ \\
\hline Temperature (K) & 150 \\
\hline$a, b, c(\AA)$ & 14.0218 (8), 26.5291 (14), 20.8174 (11) \\
\hline$\beta\left({ }^{\circ}\right)$ & $94.111(3)$ \\
\hline$V\left(\AA^{3}\right)$ & $7723.9(7)$ \\
\hline$Z$ & 4 \\
\hline Radiation type & Mo Ka \\
\hline$\mu\left(\mathrm{mm}^{-1}\right)$ & 0.31 \\
\hline Crystal size $(\mathrm{mm})$ & $0.50 \times 0.30 \times 0.03$ \\
\hline \multicolumn{2}{|l|}{ Data collection } \\
\hline Diffractometer & Bruker AXS APEXII Kappa CCD diffractometer \\
\hline Absorption correction & $\begin{array}{l}\text { Multi-scan SADABS 2016/2: Krause, L., Herbst-Irmer, R., Sheldrick } \\
\text { G.M. \& Stalke D., J. Appl. Cryst. } 48 \text { (2015) 3-10 }\end{array}$ \\
\hline$T_{\min }, T_{\max }$ & $0.194,0.274$ \\
\hline $\begin{array}{l}\text { No. of measured, independent and } \\
\text { observed }[I>2 s(I)] \text { reflections }\end{array}$ & $192200,19154,8488$ \\
\hline$R_{\text {int }}$ & 0.170 \\
\hline$(\sin \theta / \lambda)_{\max }\left(\AA^{-1}\right)$ & 0.667 \\
\hline \multicolumn{2}{|l|}{ Refinement } \\
\hline$R\left[F^{2}>2 \sigma\left(F^{2}\right)\right], w R\left(F^{2}\right), S$ & $0.096,0.289,1.02$ \\
\hline No. of reflections & 19154 \\
\hline No. of parameters & 1379 \\
\hline No. of restraints & 2002 \\
\hline \multirow[t]{2}{*}{$\mathrm{H}$-atom treatment } & $\mathrm{H}$-atom parameters constrained \\
\hline & $w=1 /\left[\mathrm{s}^{2}\left(F_{\mathrm{o}}^{2}\right)+(0.1081 P)^{2}+11.0884 P\right]$ where $P=\left(F_{\mathrm{o}}^{2}+2 F_{\mathrm{c}}^{2}\right) / 3$ \\
\hline$\Delta \rho_{\max }, \Delta \rho_{\min }\left(\mathrm{e} \AA^{-3}\right)$ & $0.47,-0.36$ \\
\hline
\end{tabular}

Computer programs: Apex3 v2017.3-0 (Bruker, 2016), SAINT V8.38A (Bruker, 2016), SHELXS97

(Sheldrick, 2008), SHELXL2018/3 (Sheldrick, 2015, 2018), SHELXLE Rev937 (Hübschle et al., 2011). 


\section{Refinement details:}

A benzyl group bound to the pyridyl backbone of the ligand is disordered by rotation. The disorder extends to the borane fragment of a neighboring molecule. For both the pyridyl/benzyl as well as the disordered borane unit the major and minor moieties were restrained to have similar geometries. The minor benzyl moiety and the minor pyridyl moiety were restrained to be close to planar. $\mathrm{U}^{\mathrm{ij}}$ components of ADPs for disordered atoms closer to each other than $2.0 \AA$ were restrained to be similar. Subject to these conditions the occupancy ratio refined to $0.834(5)$ to $0.166(5)$.

The K(2,2,2-crypt) cation was refined as disordered over two orientations, distinguished by folding of some of the ethyl-ether units. The potassium ion was excluded from the disorder. The two disordered moieties were restrained to have similar geometries. $\mathrm{U}^{\mathrm{ij}}$ components of ADPs for disordered atoms closer to each other than $2.0 \AA$ were restrained to be similar. Subject to these conditions the occupancy ratio refined to $0.878(5)$ to $0.122(5)$.

Both THF solvate molecules were refined as disordered over each two orientations. All THF moieties were restrained to have similar geometries. $U^{\mathrm{ij}}$ components of ADPs for disordered atoms closer to each other than $2.0 \AA$ A were restrained to be similar. Subject to these conditions the occupancy ratio refined to $0.279(14)$ to $0.721(14)$ (THF of O7) and $0.674(8)$ to $0.326(8)$ (THF of O8).

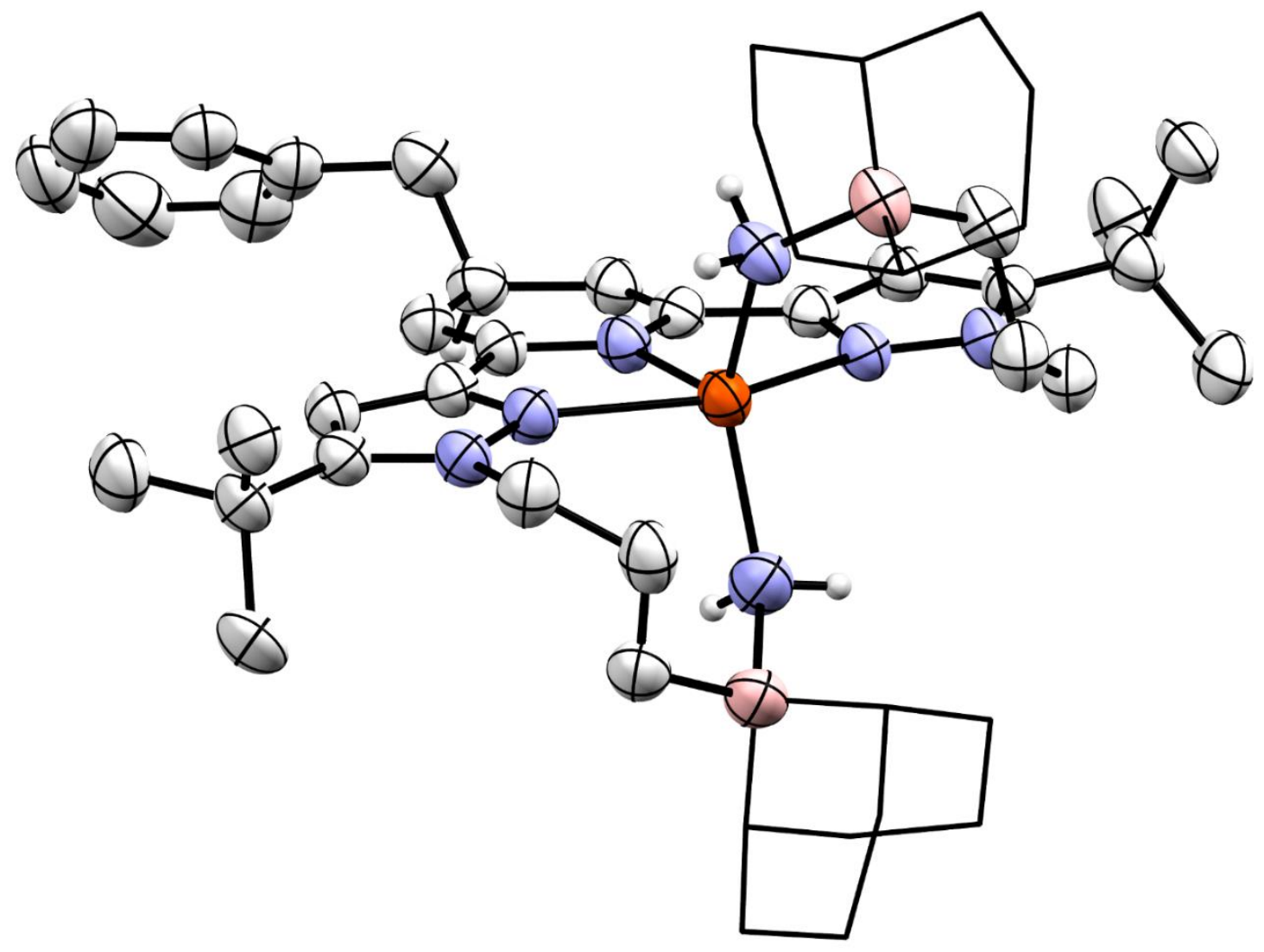

Figure S98 Molecular structure of $\left[\mathrm{K}\left(2,2,2\right.\right.$-cryptand)][(benzyl- $\left.\left.{ }^{\mathrm{BBN}} \mathrm{PDP}{ }^{\mathrm{tBu}}\right) \mathrm{Fe}\left(\mathrm{NH}_{2}\right)_{2}\right]$ displayed with $50 \%$ probability ellipsoids. Most hydrogen atoms not attached to heteroatoms as well as solvate molecules and disorder are omitted for clarity. The cationic portion of the molecule is omitted for clarity. The 9BBN substituents are displayed in wireframe for clarity. 
Complex: $\left[\mathrm{Cs}(18-\text { crown-6) })_{2}\right]\left[\left({ }^{\mathrm{BBN} P D P} \mathrm{P}^{\mathrm{tBu}}\right) \mathrm{Co}\left(\mathrm{NH}_{2}\right)_{2}\right]$

Local name: jk565

CCDC 1993637

Table S21 Crystallographic experimental parameters for [Cs(18-crown-6 $\left.)_{2}\right]\left[\left({ }^{B B N} P^{2 B P}\right) \mathrm{Co}\left(\mathrm{NH}_{2}\right)_{2}\right]$.

\begin{tabular}{|c|c|}
\hline \multicolumn{2}{|l|}{ Crystal data } \\
\hline Chemical formula & $\mathrm{C}_{41} \mathrm{H}_{67} \mathrm{~B}_{2} \mathrm{CoN}_{7} \cdot \mathrm{C}_{24} \mathrm{H}_{48} \mathrm{CsO}_{12}$ \\
\hline$M r$ & 1400.09 \\
\hline Crystal system, space group & Hexagonal, $P 6_{1} 22$ \\
\hline Temperature (K) & 150 \\
\hline$a, c(\AA)$ & $14.1962(10), 60.572(5)$ \\
\hline$V\left(\AA^{3}\right)$ & $10571.8(17)$ \\
\hline$Z$ & 6 \\
\hline Radiation type & $\mathrm{Cu} K \alpha$ \\
\hline$\mu\left(\mathrm{mm}^{-1}\right)$ & 6.37 \\
\hline Crystal size (mm) & $0.12 \times 0.11 \times 0.03$ \\
\hline \multicolumn{2}{|l|}{ Data collection } \\
\hline Diffractometer & $\begin{array}{l}\text { Bruker AXS D8 Quest CMOS diffractometer with Photonll charge- } \\
\text { integrating pixel array detector (CPAD) }\end{array}$ \\
\hline Absorption correction & $\begin{array}{l}\text { Multi-scan SADABS 2016/2: Krause, L., Herbst-Irmer, R., Sheldrick G.M. } \\
\text { \& Stalke D., J. Appl. Cryst. } 48 \text { (2015) 3-10 }\end{array}$ \\
\hline$T_{\min }, T_{\max }$ & $0.456,0.754$ \\
\hline $\begin{array}{l}\text { No. of measured, independent and } \\
\text { observed }[I>2 s(I)] \text { reflections }\end{array}$ & $36938,7507,6496$ \\
\hline$R_{\text {int }}$ & 0.072 \\
\hline$(\sin \theta / \lambda)_{\max }\left(\AA^{-1}\right)$ & 0.637 \\
\hline \multicolumn{2}{|l|}{ Refinement } \\
\hline$R\left[F^{2}>2 \sigma\left(F^{2}\right)\right], w R\left(F^{2}\right), S$ & $0.042,0.112,1.06$ \\
\hline No. of reflections & 7507 \\
\hline No. of parameters & 514 \\
\hline No. of restraints & 356 \\
\hline $\mathrm{H}$-atom treatment & H-atom parameters constrained \\
\hline$\Delta \rho_{\max }, \Delta \rho_{\min }\left(\mathrm{e} \AA^{-3}\right)$ & $0.39,-1.24$ \\
\hline Absolute structure & $\begin{array}{l}\text { Flack x determined using } 2253 \text { quotients }[(I+)-(I-)] /[(I+)+(I-)] \text { (Parsons, } \\
\text { Flack and Wagner, Acta Cryst. B69 (2013) 249-259). }\end{array}$ \\
\hline Absolute structure parameter & $-0.004(3)$ \\
\hline
\end{tabular}

Computer programs: Apex3 v2017.3-0 (Bruker, 2017), SAINT V8.38A (Bruker, 2016), SHELXS97 (Sheldrick, 2008), SHELXL2018/3 (Sheldrick, 2015, 2018), SHELXLE Rev1030 (Hübschle et al., 2011). 


\section{Refinement details:}

The structure was solved by isomorphous replacement from its Fe counterpart, plus changing to its enantiomorph space group ( $P 6_{5} 22$ for the iron crystal, $P 6_{1} 22$ for the cobalt crystal).

The borolene fragment and surrounding atoms are disordered, with differing directions of rotation that the fragment wraps around the cobalt center. The two disordered moieties were restrained to have similar geometries. Major and minor atoms C11 were restrained to be coplanar with the adjacent to the $\mathrm{N}_{2} \mathrm{C}_{3}$ ring. $\mathrm{U}^{\mathrm{ij}}$ components of ADPs for disordered atoms closer to each other than $2.0 \AA$ were restrained to be similar. Subject to these conditions the occupancy ratio refined to $0.856(4)$ to $0.144(4)$.

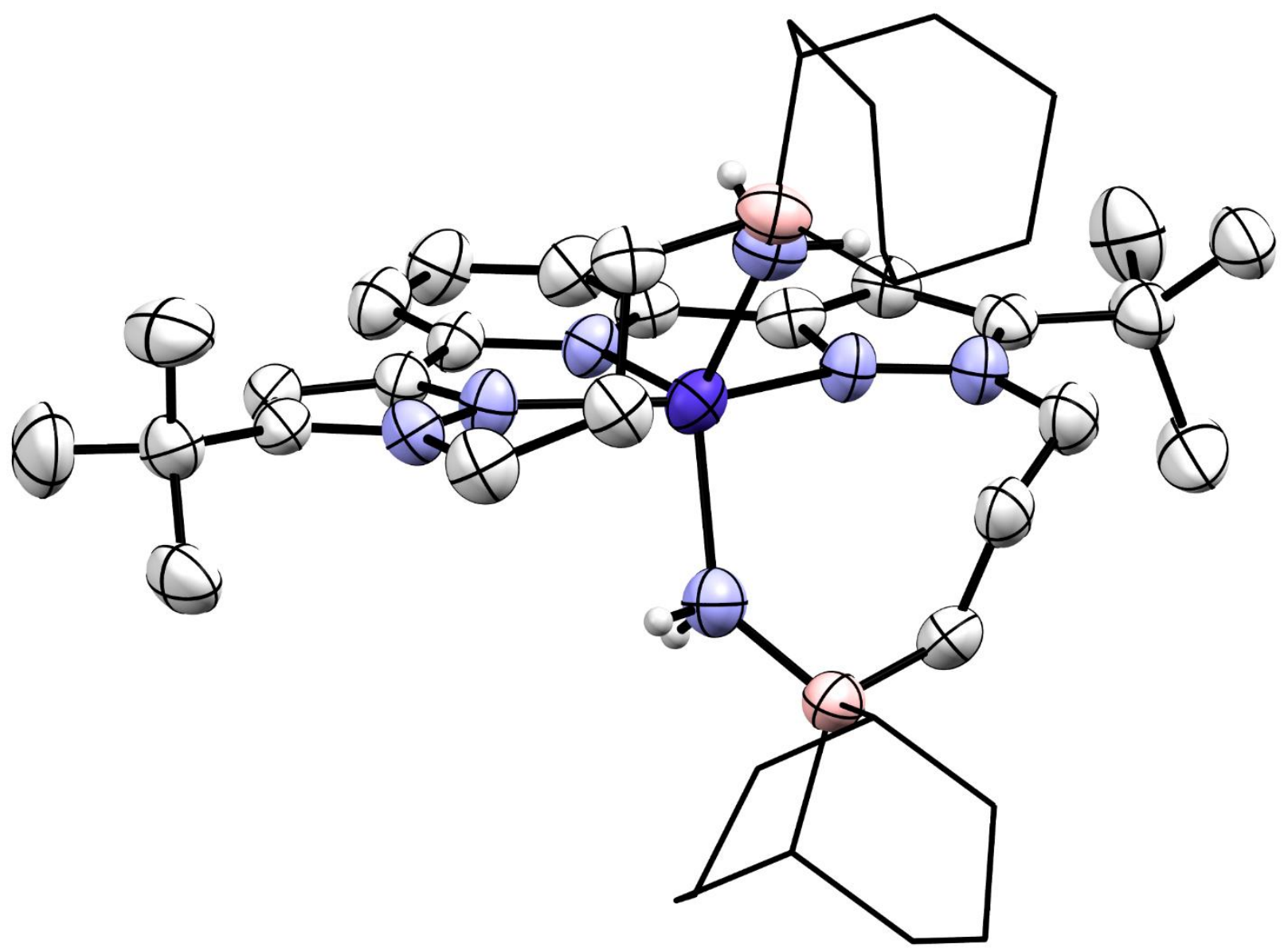

Figure S99 Molecular structure of [Cs(18-crown-6 $\left.)_{2}\right]\left[\left({ }^{B B N} \mathrm{PDP}{ }^{\mathrm{BBu}}\right) \mathrm{Co}\left(\mathrm{NH}_{2}\right)_{2}\right]$ displayed with $50 \%$ probability ellipsoids. Hydrogen atoms not attached to heteroatoms are omitted for clarity. The cationic portion of the molecule and disorder are omitted for clarity. The 9-BBN substituents are displayed in wireframe for clarity. 
Complex: (benzyl- $\left.{ }^{\mathrm{BBN}} \mathrm{PDP}{ }^{\mathrm{BBu}}\right) \mathrm{Fe}\left(\mathrm{NH}_{2}\right)_{2}$

Local name: jk572

CCDC 1993642

Table S22 Crystallographic experimental parameters for (benzyl- $\left.{ }^{\mathrm{BBN}} \mathrm{PDP}{ }^{\mathrm{tBu}}\right) \mathrm{Fe}\left(\mathrm{NH}_{2}\right)_{2}$.

\begin{tabular}{|c|c|}
\hline \multicolumn{2}{|l|}{ Crystal data } \\
\hline Chemical formula & $\mathrm{C}_{48} \mathrm{H}_{73} \mathrm{~B}_{2} \mathrm{FeN} \cdot 2\left(\mathrm{C}_{7} \mathrm{H}_{8}\right)$ \\
\hline Mr & 1009.87 \\
\hline Crystal system, space group & Triclinic, $P \overline{1}$ \\
\hline Temperature (K) & 150 \\
\hline$a, b, c(\AA)$ & 12.5395 (7), $13.9281(8), 17.4233(10)$ \\
\hline$\alpha, \beta, \gamma\left({ }^{\circ}\right)$ & $75.712(2), 80.915(2), 78.172(2)$ \\
\hline$V\left(\AA^{3}\right)$ & $2867.7(3)$ \\
\hline$z$ & 2 \\
\hline Radiation type & Mo $K \alpha$ \\
\hline$\mu\left(\mathrm{mm}^{-1}\right)$ & 0.31 \\
\hline Crystal size $(\mathrm{mm})$ & $0.38 \times 0.21 \times 0.08$ \\
\hline \multicolumn{2}{|l|}{ Data collection } \\
\hline Diffractometer & Bruker AXS D8 Quest CMOS diffractometer \\
\hline Absorption correction & $\begin{array}{l}\text { Multi-scan SADABS 2016/2: Krause, L., Herbst-Irmer, R., Sheldrick } \\
\text { G.M. \& Stalke D., J. Appl. Cryst. } 48 \text { (2015) 3-10 }\end{array}$ \\
\hline$T_{\min }, T_{\max }$ & $0.677,0.747$ \\
\hline $\begin{array}{l}\text { No. of measured, independent and } \\
\text { observed }[I>2 \mathrm{~s}(I)] \text { reflections }\end{array}$ & $190895,21957,18296$ \\
\hline$R_{\text {int }}$ & 0.035 \\
\hline$(\sin \theta / \lambda)_{\max }\left(\AA^{-1}\right)$ & 0.773 \\
\hline \multicolumn{2}{|l|}{ Refinement } \\
\hline$R\left[F^{2}>2 \sigma\left(F^{2}\right)\right], w R\left(F^{2}\right), S$ & $0.043,0.113,1.06$ \\
\hline No. of reflections & 21957 \\
\hline No. of parameters & 669 \\
\hline No. of restraints & 4 \\
\hline $\mathrm{H}$-atom treatment & $\begin{array}{l}H \text { atoms treated by a mixture of independent and constrained } \\
\text { refinement }\end{array}$ \\
\hline$\Delta \rho_{\max }, \Delta \rho_{\min }\left(\mathrm{e} \AA^{-3}\right)$ & $0.61,-0.53$ \\
\hline
\end{tabular}

Computer programs: Apex3 v2018.1-0 (Bruker, 2018), SAINT V8.38A (Bruker, 2016), SHELXS97

(Sheldrick, 2008), SHELXL2018/3 (Sheldrick, 2015, 2018), SHELXLE Rev946 (Hübschle et al., 2011). 


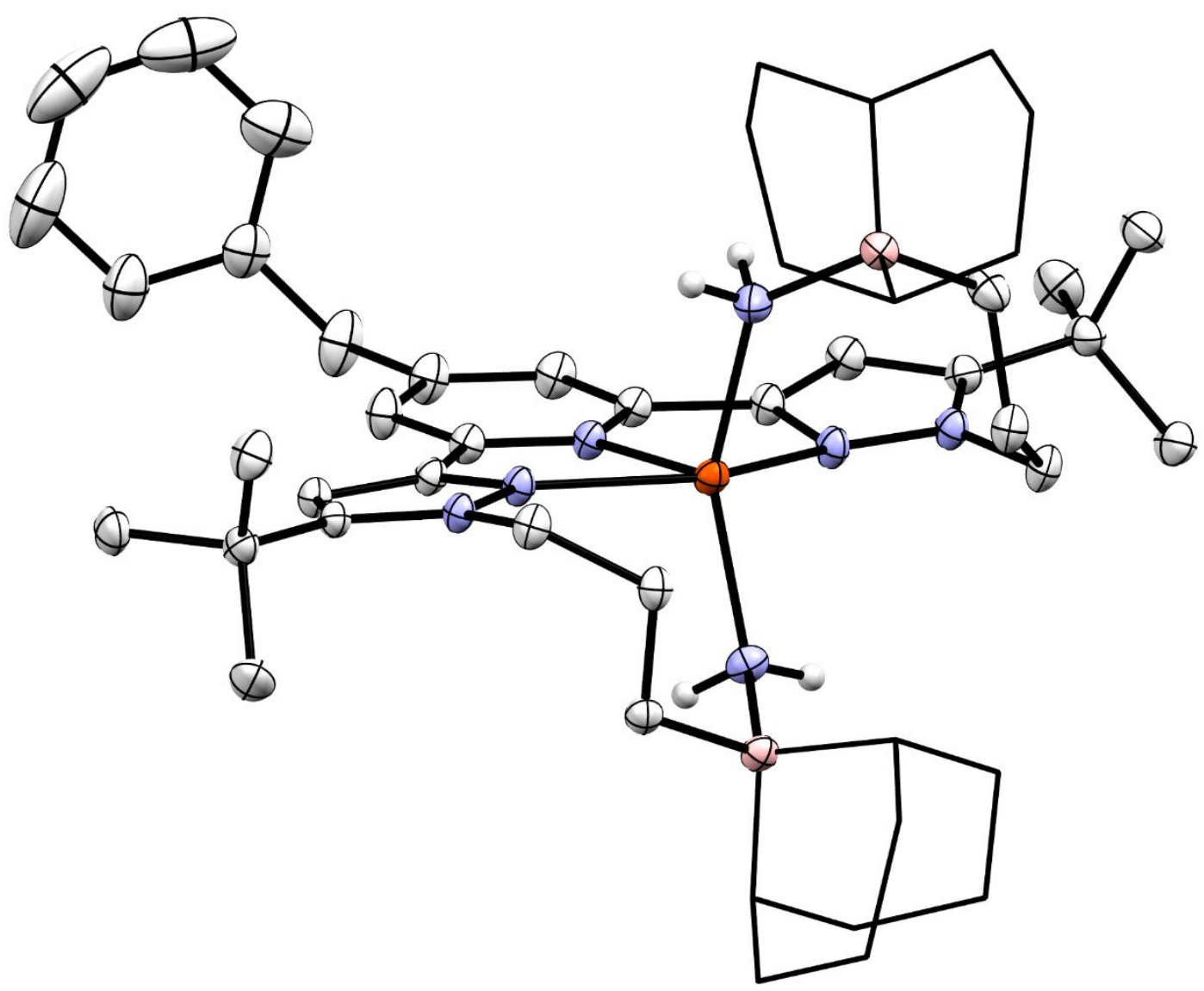

Figure $\mathbf{S 1 0 0}$ Molecular structure of (benzyl- $\left.{ }^{B B N} P D P{ }^{t B u}\right) \mathrm{Fe}\left(\mathrm{NH}_{2}\right)_{2}$ displayed with $50 \%$ probability ellipsoids. Hydrogen atoms not attached to heteroatoms as well as solvate molecules are omitted for clarity. The 9BBN substituents are displayed in wireframe for clarity. 
Complex: $\left[\left({ }^{\mathrm{BBN} P D P} \mathrm{PBu}^{\mathrm{Bu}}\right) \mathrm{Fe}\left(\mathrm{NH}_{2}\right)_{2}\right]_{2}[\mathrm{Li}(\mathrm{THF})(12-\mathrm{crown}-4)]_{2}$

Local name: jk5100

CCDC 1993630

Table S23 Crystallographic experimental parameters for $\left[\left({ }^{\mathrm{BBN} P D P}{ }^{\mathrm{BBu}}\right) \mathrm{Fe}\left(\mathrm{NH}_{2}\right)_{2}\right]_{2}[\mathrm{Li}(\mathrm{THF})(12-\mathrm{crown}-4)]_{2}$.

\begin{tabular}{|c|c|}
\hline \multicolumn{2}{|l|}{ Crystal data } \\
\hline Chemical formula & $\mathrm{C}_{82} \mathrm{H}_{132} \mathrm{~B}_{4} \mathrm{Fe}_{2} \mathrm{~N}_{14} \cdot 2\left(\mathrm{C}_{12} \mathrm{H}_{24} \mathrm{LiO}_{5}\right) \cdot 16\left(\mathrm{C}_{4} \mathrm{H}_{8} \mathrm{O}\right)$ \\
\hline$M r$ & 3133.11 \\
\hline Crystal system, space group & Triclinic, $P \overline{1}$ \\
\hline Temperature (K) & 150 \\
\hline$a, b, c(\AA)$ & $15.6959(10), 17.5755(13), 19.4829$ (12) \\
\hline$\alpha, \beta, \gamma\left({ }^{\circ}\right)$ & $112.269(2), 103.330(3), 104.655$ (3) \\
\hline$V\left(\AA^{3}\right)$ & $4480.7(5)$ \\
\hline$Z$ & 1 \\
\hline Radiation type & Mo $K \alpha$ \\
\hline$\mu\left(\mathrm{mm}^{-1}\right)$ & 0.23 \\
\hline Crystal size (mm) & $0.22 \times 0.18 \times 0.12$ \\
\hline \multicolumn{2}{|l|}{ Data collection } \\
\hline Diffractometer & Bruker AXS D8 Quest CMOS diffractometer \\
\hline Absorption correction & $\begin{array}{l}\text { Multi-scan SADABS 2016/2: Krause, L., Herbst-Irmer, R., Sheldrick } \\
\text { G.M. \& Stalke D., J. Appl. Cryst. } 48 \text { (2015) 3-10 }\end{array}$ \\
\hline$T_{\min }, T_{\max }$ & $0.677,0.746$ \\
\hline $\begin{array}{l}\text { No. of measured, independent and } \\
\text { observed }[I>2 s(I)] \text { reflections }\end{array}$ & $70232,21556,15771$ \\
\hline$R_{\text {int }}$ & 0.050 \\
\hline$(\sin \theta / \lambda)_{\max }\left(\AA^{-1}\right)$ & 0.668 \\
\hline \multicolumn{2}{|l|}{ Refinement } \\
\hline$R\left[F^{2}>2 \sigma\left(F^{2}\right)\right], w R\left(F^{2}\right), S$ & $0.073,0.231,1.06$ \\
\hline No. of reflections & 21556 \\
\hline No. of parameters & 1881 \\
\hline No. of restraints & 6340 \\
\hline $\mathrm{H}$-atom treatment & $\mathrm{H}$-atom parameters constrained \\
\hline$\Delta \rho_{\max }, \Delta \rho_{\min }\left(\mathrm{e} \AA^{-3}\right)$ & $0.90,-0.52$ \\
\hline
\end{tabular}

Computer programs: Apex3 v2018.1-0 (Bruker, 2018), SAINT V8.38A (Bruker, 2016), SHELXS97

(Sheldrick, 2008), SHELXL2018/3 (Sheldrick, 2015, 2018), SHELXLE Rev946 (Hübschle et al., 2011). 


\section{Refinement details:}

The 12-crown-4 molecule and the associated lithium cations and coordinated THF molecule were refined as three-fold disordered. Seven other THF molecules were refined as two-fold disordered in general positions, and two in a 1:1 ratio around inversion centers $(013,014)$. The three disordered $12-$ crown-4 molecule moieties were restrained to have similar geometries. All disordered THF moieties were restrained to have similar geometries.

The ligand side arms of the main anion are disordered by their sense of rotation around the iron atom. The two disordered moieties were restrained to have similar geometries. $\mathrm{U}^{\mathrm{ij}}$ components of ADPs for disordered atoms closer to each other than $2.0 \AA$ A were restrained to be similar. Subject to these conditions the occupancy ratio refined to $0.9123(19)$ to $0.0877(19)$ for the ligand side arms (shared with the THF molecule of O11). The 12-crown-4 Li / THF disorder refined to an occupancy ratio of $0.513(3)$ to $0.358(3)$ to $0.129(2)$. Occupancies for the major THF moieties refined to $0.734(9)$ for the molecule of 06 , to $0.631(12$ ) for the molecule of 07 , to $0.620(14)$ for the molecule of 08 , to $0.808(6)$ for the molecule of 09, to $0.598(11)$ for the molecule of 010 , to $0.9123(19)$ for the molecule of 011 , to $0.656(7)$ for the molecule of 012 , to $0.734(9)$.

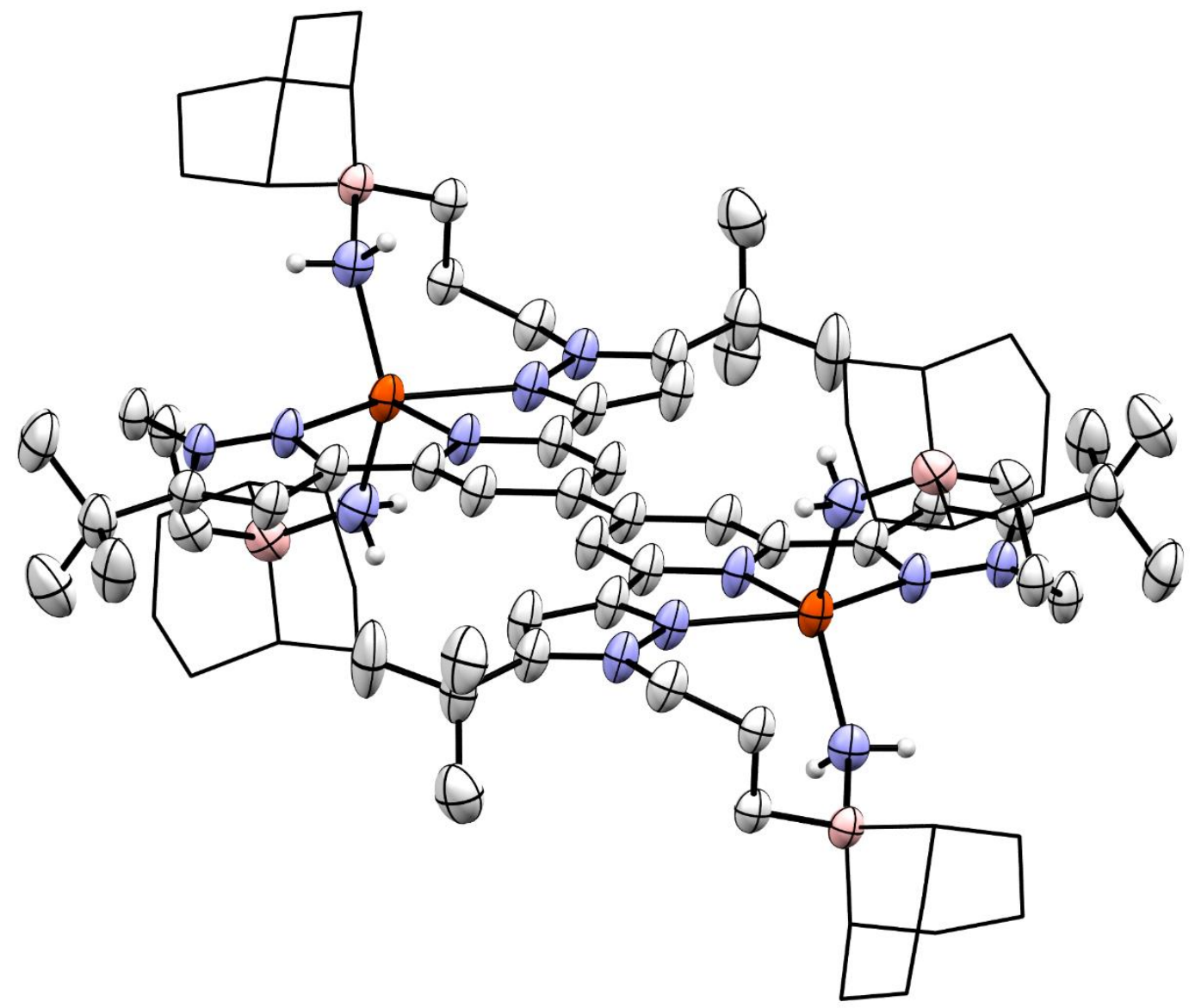

Figure S101 Molecular structure of [( $\left.\left.{ }^{\mathrm{BBN} P D P} \mathrm{P}^{\mathrm{BB} u}\right) \mathrm{Fe}\left(\mathrm{NH}_{2}\right)_{2}\right]_{2}[\mathrm{Li}(\mathrm{THF})(12-\mathrm{crown}-4)]_{2}$ displayed with $50 \%$ probability ellipsoids. Hydrogen atoms not attached to heteroatoms, minor disordered moieties and cocrystallized solvent molecules are omitted for clarity. The cationic portion of the molecule is omitted for clarity. The 9-BBN substituents are displayed in wireframe for clarity. 


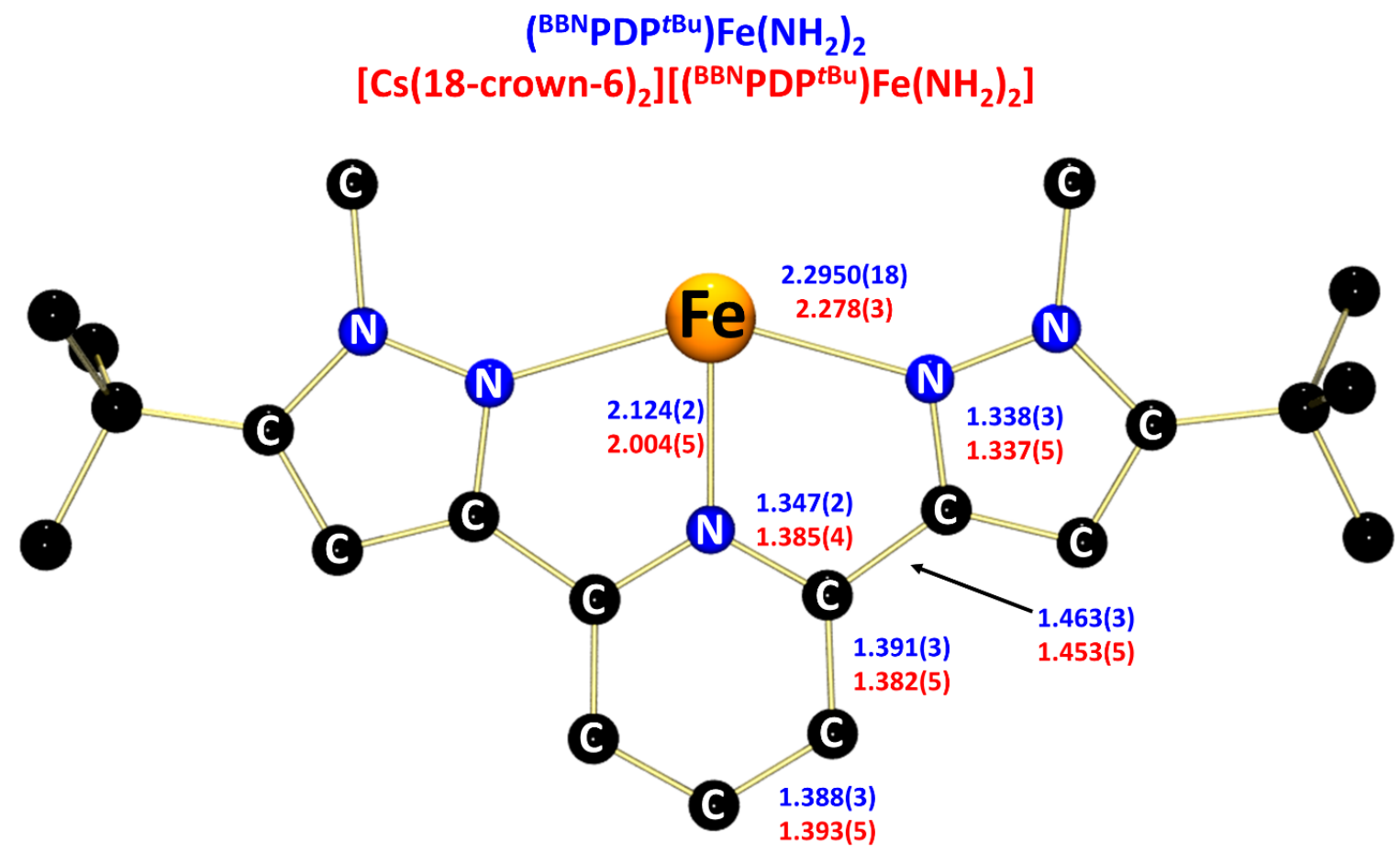

Figure S102 Comparison of experimental intraligand bond distances for ( $\left.{ }^{\mathrm{BBN} P D P}{ }^{\mathrm{BBu}}\right) \mathrm{Fe}\left(\mathrm{NH}_{2}\right)_{2}$ (blue) and $\left[\mathrm{Cs}(18-\mathrm{crown}-6)_{2}\right]\left[\left({ }^{\mathrm{BBN}} \mathrm{PDP}{ }^{\mathrm{tBu}}\right) \mathrm{Fe}\left(\mathrm{NH}_{2}\right)_{2}\right]$ (red).

\section{(BBNPDPtBu$) \mathrm{Co}\left(\mathrm{NH}_{2}\right)_{2}$ [Cs(18-crown-6) $\left.)_{2}\right]\left[\left({ }^{B B N} P D^{t B u}\right) \mathrm{Co}\left(\mathrm{NH}_{2}\right)_{2}\right]$}

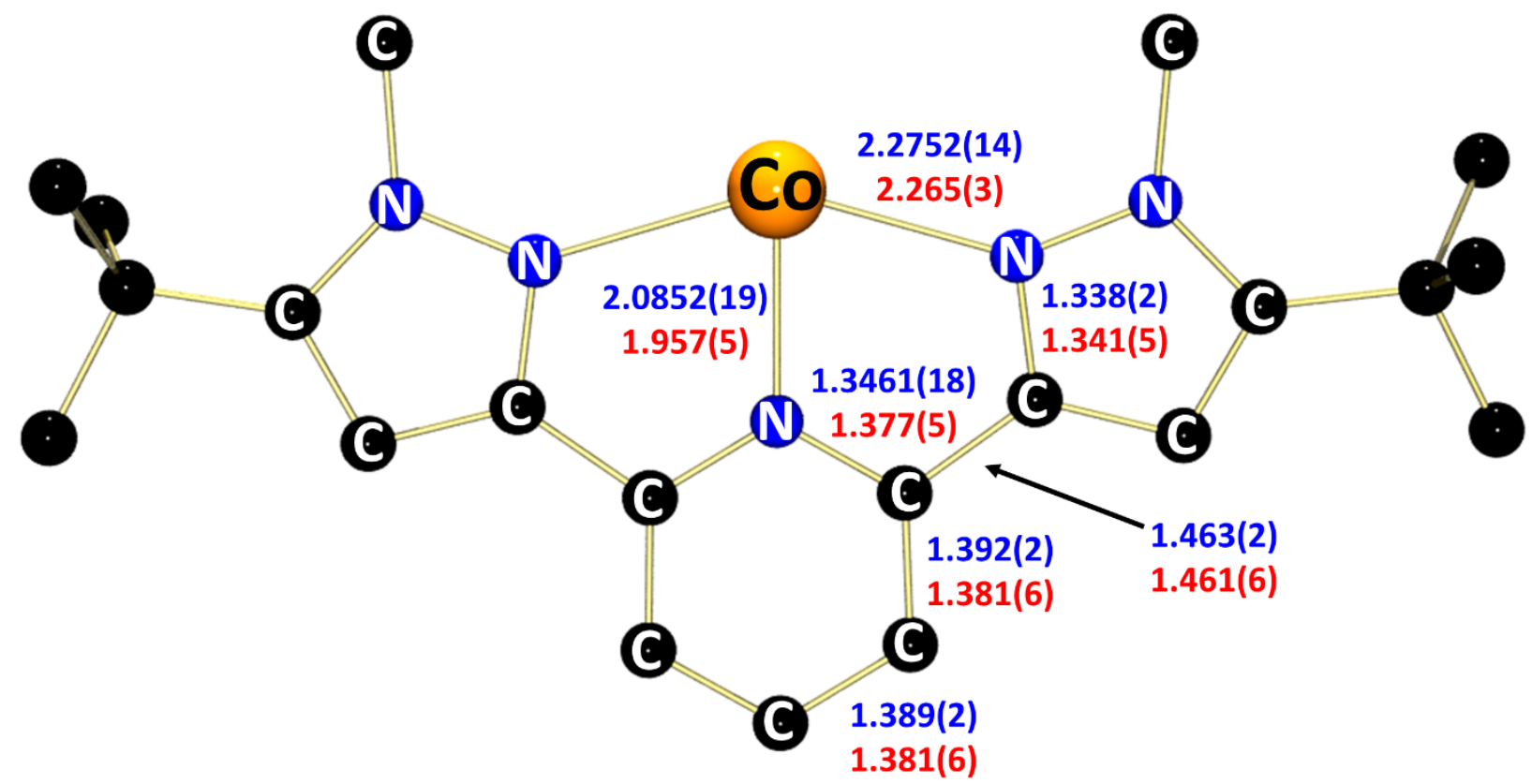

Figure S103 Comparison of experimental intraligand bond distances for ( $\left.{ }^{\mathrm{BBN} P D P}{ }^{\mathrm{BBu}}\right) \mathrm{Co}\left(\mathrm{NH}_{2}\right)_{2}($ blue) and [Cs(18-crown-6) $\left.)_{2}\right]\left[\left({ }^{\text {BBN PDP }}{ }^{\text {Bu }}\right) \mathrm{Co}\left(\mathrm{NH}_{2}\right)_{2}\right]$ (red). 


\section{( $\left.{ }^{\mathrm{BBN} P D P}{ }^{\mathrm{Bu}}\right) \mathrm{VCl}_{3}$ ( $\left.{ }^{B B N P D P t B u}\right) \mathrm{VCl}_{2}\left(\mathrm{~N}_{2} \mathrm{H}_{4}\right)(\mathrm{THF})$}

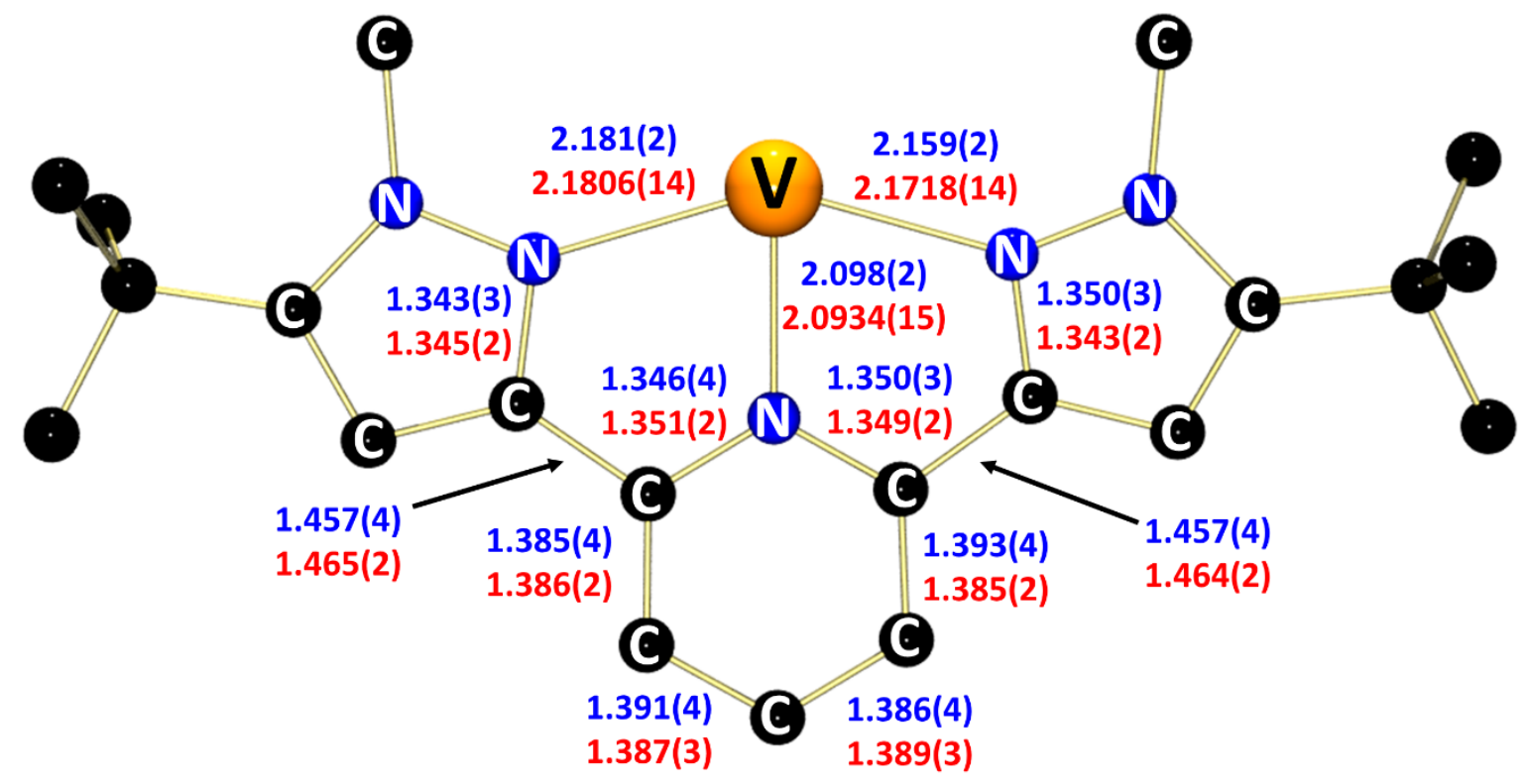

Figure S104 Comparison of experimental intraligand bond distances for ( $\left.{ }^{\mathrm{BBN}} \mathrm{PDP}^{\mathrm{tBu}}\right) \mathrm{VCl}_{3}$ (blue) and $\left({ }^{\mathrm{BBN} P D P}{ }^{\mathrm{tBu}}\right) \mathrm{VCl}_{2}\left(\mathrm{~N}_{2} \mathrm{H}_{4}\right)$ (THF) (red).

Table S24 Experimental bond distances for ammonia-borane complexes. ${ }^{a}$

\begin{tabular}{|c|c|c|c|c|}
\hline & $\left({ }^{B B N}{ }^{2} P^{t B u}\right) \mathrm{MnBr}_{2}\left(\mathrm{NH}_{3}\right)_{2}$ & $\left({ }^{\mathrm{BBN}} \mathrm{PDP}^{t \mathrm{Bu}}\right) \mathrm{FeBr}_{2}\left(\mathrm{NH}_{3}\right)_{2}$ & $\left({ }^{\mathrm{BBN}} \mathrm{PDP}^{t \mathrm{Bu}}\right) \mathrm{CoBr}_{2}\left(\mathrm{NH}_{3}\right)_{2}$ & $\left({ }^{\mathrm{BBN}} \mathrm{PDP}^{\mathrm{tBu}}\right) \mathrm{ZnCl}_{2}\left(\mathrm{NH}_{3}\right)_{2}$ \\
\hline M-X1 & $2.5180(4)$ & $2.4772(4)$ & $2.4370(4)$ & $2.2805(4)$ \\
\hline$M-X 2$ & $2.4872(4)$ & $2.4189(5)$ & $2.4062(4)$ & $2.2314(5)$ \\
\hline$M-N_{\text {pyrazole }}$ & $2.3094(19)$ & $2.2608(19)$ & $2.2388(19)$ & $2.3019(14)$ \\
\hline$M-N_{\text {pyrazole }}$ & $2.3106(19)$ & $2.2776(19)$ & $2.2403(19)$ & $2.2635(13)$ \\
\hline$M-N_{\text {pyridine }}$ & 2.1994(19) & $2.0972(19)$ & $2.0581(19)$ & $2.0884(13)$ \\
\hline $\mathrm{B} 1-\mathrm{NH}_{3}$ & $1.658(4)$ & $1.660(4)$ & $1.666(4)$ & $1.660(3)$ \\
\hline B2-NH ${ }_{3}$ & $1.630(3)$ & $1.633(4)$ & $1.632(4)$ & $1.638(6)$ \\
\hline$\Sigma B_{1 \alpha}$ & $320.2(2)$ & $320.3(2)$ & $320.2(2)$ & $328.4(6)$ \\
\hline$\Sigma B_{2 \alpha}$ & $318.9(2)$ & $319.2(2)$ & $318.9(2)$ & $319.6(4)$ \\
\hline$\tau_{5}$ & 0.11 & 0.18 & 0.21 & 0.24 \\
\hline
\end{tabular}

${ }^{\mathrm{a}}$ The $\mathrm{Mn}, \mathrm{Fe}$, and Co species crystallize with two independent molecules per unit cell. Data is reported for those of molecule 1. See cif for data of other independent molecule. 
Table S25 Experimental bond distances for hydrazine captured divalent compounds.

\begin{tabular}{lccccc} 
& $(\mathbf{L}) \mathbf{V C l}_{2}\left(\mathbf{N}_{2} \mathrm{H}_{4}\right)(\mathrm{THF})$ & $(\mathbf{L}) \mathrm{MnBr}_{2}\left(\mathbf{N}_{2} \mathrm{H}_{4}\right)$ & $(\mathbf{L}) \mathrm{FeBr}_{2}\left(\mathrm{~N}_{2} \mathrm{H}_{4}\right)^{\mathrm{a}}$ & $(\mathbf{L}) \mathrm{CoBr}_{2}\left(\mathbf{N}_{2} \mathrm{H}_{4}\right)$ & $(\mathbf{L}) \mathbf{Z n C l}_{2}\left(\mathrm{~N}_{2} \mathrm{H}_{4}\right)^{\mathrm{a}}$ \\
\hline $\mathbf{M}-\mathbf{X 1}$ & $2.4715(5)$ & $2.5387(6)$ & $2.4879(3)$ & $2.4523(6)$ & $2.2708(7)$ \\
$\mathbf{M}-\mathbf{X 2}$ & $2.4799(5)$ & $2.5219(5)$ & $2.4618(3)$ & $2.4324(6)$ & $2.2815(7)$ \\
$\mathbf{M}-\mathbf{N}_{\text {pyrazole }}$ & $2.1806(14)$ & $2.272(3)$ & $2.2331(13)$ & $2.197(3)$ & $2.264(2)$ \\
$\mathbf{M}-\mathbf{N}_{\text {pyrazole }}$ & $2.1718(14)$ & $2.275(3)$ & $2.2279(13)$ & $2.208(3)$ & $2.304(2)$ \\
$\mathbf{M}-\mathbf{N}_{\text {pyridine }}$ & $2.0934(15)$ & $2.190(2)$ & $2.0983(13)$ & $2.048(2)$ & $2.087(2)$ \\
$\mathbf{B 1 - N}$ & $1.759(2)$ & $1.688(5)$ & $1.697(2)$ & $1.697(4)$ & $1.696(4)$ \\
$\mathbf{B 2 - N}$ & $1.705(3)$ & $1.694(5)$ & $1.698(2)$ & $1.699(4)$ & $1.682(4)$ \\
$\mathbf{N}-\mathbf{N}$ & $1.478(2)$ & $1.479(3)$ & $1.4681(17)$ & $1.463(3)$ & $1.475(3)$ \\
$\mathbf{\Sigma} \mathbf{B}_{1 \alpha}$ & $323.01(15)$ & $320.6(3)$ & $318.88(12)$ & $318.3(2)$ & $321.1(2)$ \\
$\mathbf{\Sigma} \mathbf{B}_{2 \alpha}$ & $321.27(15)$ & $319.2(3)$ & $319.96(13)$ & $319.0(3)$ & $321.4(2)$ \\
$\tau_{5}$ & -- & 0.34 & 0.42 & 0.44 & 0.39 \\
\hline
\end{tabular}

a Data from J. Am. Chem. Soc. 2017, 139, 18194-18197 for comparison

Table S26 Experimental bond distance for vanadium compounds.

\begin{tabular}{|c|c|c|c|}
\hline & $\left({ }^{\mathrm{BBN}} \mathrm{PDP}^{\mathrm{tBu}}\right) \mathrm{VCl}_{3}$ & (L) $\mathrm{VCl}_{2}\left(\mathrm{~N}_{2} \mathrm{H}_{4}\right)$ (THF) & $(\mathrm{L}) \mathrm{VCl}_{2}\left(\mathrm{NH}_{3}\right)_{2}$ (THF) \\
\hline V-Cl1 & $2.3359(8)$ & $2.4715(5)$ & $2.4714(6)$ \\
\hline $\mathrm{V}-\mathrm{Cl} 2$ & $2.3041(7)$ & $2.4799(5)$ & $2.4737(6)$ \\
\hline $\mathrm{V}-\mathrm{Cl} 3$ & $2.3492(8)$ & -- & -- \\
\hline M-N $N_{\text {pyrazole }}$ & $2.181(2)$ & $2.1806(14)$ & $2.2241(19)$ \\
\hline M-N pyrazole & $2.159(2)$ & $2.1718(14)$ & $2.2127(18)$ \\
\hline M-N $N_{\text {pyridine }}$ & $2.098(2)$ & $2.0934(15)$ & $2.1007(17)$ \\
\hline B1-N & -- & $1.759(2)$ & $1.640(3)$ \\
\hline B2-N & -- & $1.705(3)$ & $1.665(3)$ \\
\hline$\Sigma B_{1 \alpha}$ & $359.8(6)$ & $323.01(15)$ & $320.82(18)$ \\
\hline$\Sigma B_{2 \alpha}$ & $359.8(3)$ & $321.27(15)$ & $320.13(18)$ \\
\hline
\end{tabular}

Table S27 Experimental bond distances for amido-borane complexes.

\begin{tabular}{|c|c|c|c|c|c|}
\hline & $\left({ }^{B B N P D P}{ }^{t B u}\right) \mathrm{Mn}\left(\mathrm{NH}_{2}\right)_{2}$ & $\left({ }^{B B N} P D P{ }^{t B u}\right) F e\left(N_{2}\right)_{2}{ }^{a}$ & $\left({ }^{B B N} P D P P^{t B u}\right) \mathrm{Co}\left(\mathrm{NH}_{2}\right)_{2}$ & $\left({ }^{B B N} P D P^{t B u}\right) Z n\left(N_{2}\right)_{2}$ & {$\left[\left({ }^{\mathrm{BBN} P D P}{ }^{t \mathrm{Bu}}\right) \mathrm{Co}\left(\mathrm{NH}_{2}\right)_{2}\right]^{1-}$} \\
\hline $\mathrm{M}-\mathrm{NH}_{2}$ & $2.1364(17)$ & $2.075(2)$ & $2.0411(17)$ & $2.006(2)$ & $2.066(7)$ \\
\hline$M-N_{\text {pyrazole }}$ & $2.3345(16)$ & $2.2950(18)$ & $2.2752(14)$ & $2.331(2)$ & $2.265(3)$ \\
\hline$M-N_{\text {pyridine }}$ & $2.240(2)$ & $2.124(2)$ & $2.0852(19)$ & $2.141(3)$ & $1.957(5)$ \\
\hline B- $\mathrm{NH}_{2}$ & $1.625(3)$ & $1.633(3)$ & $1.633(3)$ & $1.619(4)$ & $1.622(9)$ \\
\hline$\Sigma \mathbf{B}_{\alpha}$ & $325.65(18)$ & $325.6(2)$ & $326.09(16)$ & $325.9(2)$ & $325.8(5)$ \\
\hline$\tau_{5}$ & 0.07 & 0.02 & 0.08 & 0.03 & 0.36 \\
\hline
\end{tabular}

${ }^{a}$ Data from J. Am. Chem. Soc. 2017, 139, 18194-18197 for comparison 
Table S28 Experimental bond distances for Fe amido-borane complexes.

\begin{tabular}{|c|c|c|c|c|c|}
\hline & $\left(^{B B N P D P t B u}\right) \mathrm{Fe}\left(\mathrm{NH}_{2}\right)_{2}{ }^{\mathrm{a}}$ & {$\left[\left(^{B B N P D P}{ }^{t B u}\right) F e\left(\mathrm{NH}_{2}\right)_{2}\right]^{1-}$} & {$\left[(\text { benzyl-BBNPDPtBu}) \mathrm{Fe}\left(\mathrm{NH}_{2}\right)_{2}\right]^{1-}$} & (benzyl-BBNPDP $\left.{ }^{\mathrm{tBu}}\right) \mathrm{Fe}\left(\mathrm{NH}_{2}\right)_{2}$ & {$\left[\left({ }^{\left.B B N P D P^{\mathrm{Bu}}\right)}\right) \mathrm{Fe}\left(\mathrm{NH}_{2}\right)_{2}\right]_{2^{2-}}$} \\
\hline Fe-N $N_{\text {pyridine }}$ & $2.124(2)$ & $2.004(5)$ & $2.021(4)$ & $2.1194(8)$ & $2.0266(15)$ \\
\hline B1-NH 2 & $1.633(3)$ & $1.616(9)$ & $1.614(7) / 1.632(11)$ & $1.6341(15) / 1.6275(13)$ & $1.600(3) / 1.627(3)$ \\
\hline$\Sigma \mathbf{B}_{\alpha}$ & $325.6(2)$ & $325.0(5)$ & $325.3(4) / 323.4(8)$ & $325.45(9) / 325.77(8)$ & $325.7(2) / 325.85(19)$ \\
\hline$\tau_{5}$ & 0.02 & 0.31 & 0.19 & 0.02 & 0.20 \\
\hline
\end{tabular}

${ }^{a}$ Data from J. Am. Chem. Soc. 2017, 139, 18194-18197 for comparison

\section{References}

${ }^{1}$ Chakraborty, S.; Chattopadhyay, J.; Guo, W.; Billups, W. E., Functionalization of Potassium Graphite. Angew. Chem. Int. Ed. 2007, 46, 4486-4488.

2 Johnson, S. A.; Kiernicki, J. J.; Fanwick, P. E.; Bart, S. C., New Benzylpotassium Reagents and Their Utility for the Synthesis of Homoleptic Uranium(IV) Benzyl Derivatives. Organometallics, 2015, 34, 2889-2895.

${ }^{3}$ Tao, J.; Tran, R.; Murphy, G. K., Dihaloiodoarenes: $\alpha, \alpha$-Dihalogenation of Phenylacetate Derivatives. J. Am. Chem. Soc. 2013, 135, 16312-16315.

4 Jordan, R. F.; Echols, S. F., Synthesis and chemistry of $\left[\mathrm{Cp}_{2} \mathrm{Zr}\left(\mathrm{CH}_{3} \mathrm{CN}\right)_{3}\right]\left[\mathrm{BPh}_{4}\right]_{2}$ : a five-coordinate, dicationic zirconocene complex. Inorg. Chem. 1987, 26, 383-386.

${ }^{5}$ Kiernicki, J. J.; Zeller, M.; Szymczak, N. K., Hydrazine Capture and N-N Bond Cleavage at Iron Enabled by Flexible Appended Lewis Acids. J. Am. Chem. Soc. 2017, 139, 18194-18197.

${ }^{6}$ Garland, C. W.; Nibler, J. W.; Shoemaker, D. P. Experiments in Physical Chemistry, 8th Ed.; McGraw-Hill; New York: 2003, p. 371-379.

${ }^{7}$ Bruker Advanced X-ray Solution, Apex3, SAINT, SADABS, Bruker AXS Inc.: Madison (WI), USA, 2018.

${ }^{8}$ van der Sluis, P.; Spek, A. L., BYPASS: an Effective Method for the Refinement of Crystal Structures Containing Disordered Solvent Regions. Acta Crystallogr., Sect. A. 1990, 46, 194-201.

${ }^{9}$ Bruker Advanced X-ray Solutions, Saint v8.34A, (2014).

${ }^{10}$ Sheldrick, G. M., A Short History of SHELX. Acta Crystallogr., Sect. A. 2008, 64, 112-122.

${ }^{11}$ Watt, G. W.; Chrisp, J. D., Spectrophotometric Method for Determination of Hydrazine. Anal. Chem. 1952, 24, 2006-2008. 
${ }^{12}$ Wickramasinghe, L. A.; Ogawa, T.; Schrock, R. R.; Müller, P. Reduction of Dinitrogen to Ammonia Catalyzed by Molybdenum Diamido Complexes. J. Am. Chem. Soc. 2017, 139, 9132-9135. 